\title{
Hochauflösende mikroskopische und spektroskopische Untersuchungen zur strukturellen Ordnung an $\mathrm{MgO}-\mathrm{CoFeB}-G r e n z f l a ̈ c h e n$
}

\author{
Dissertation \\ zur Erlangung des mathematisch-naturwissenschaftlichen \\ Doktorgrades \\ „Doctor rerum naturalium“ \\ der Georg-August-Universität Göttingen \\ im Promotionsprogramm ProPhys \\ der Georg-August University School of Science (GAUSS)
}

vorgelegt von

Henning Schuhmann

aus Wilhelmshaven

Göttingen, 2014 
Betreuungsausschuss:

Prof. Dr. M. Seibt, IV. Physikalisches Institut, Georg-August Universität Göttingen

Prof. Dr. C. Jooß, Institut für Materialphysik, Georg-August Universität Göttingen

Mitglieder der Prüfungskommission:

Referent: Prof. Dr. M. Seibt, IV. Physikalisches Institut, Georg-August Universität Göttingen

Korreferent: Prof. Dr. C. Jooß, Institut für Materialphysik, Georg-August Universität Göttingen

Weitere Mitglieder der Prüfungskommission:

Prof. Dr. Cynthia A. Volkert, Institut für Materialphysik, Georg-August Universität Göttingen

Prof. Dr. Markus Münzenberg, Grenz- und Oberflächenphysik, Ernst Moritz Arndt Universität Greifswald

Prof. Dr. Vasily Moshnyaga, I. Physikalisches Institut, Georg-August Universität Göttingen

Prof. Dr. Hans C. Hofsäss, II. Physikalisches Institut, Georg-August Universität Göttingen

Tag der mündlichen Prüfung: 


\section{Inhaltsverzeichnis}

1. Einleitung 1

2. Einführung 7

2.1. TMR-Effekt . . . . . . . . . . . . . . . . 7

2.1.1. Spinpolarisation . . . . . . . . . . . . . . 8

2.1.2. TMR in MTJ mit kristalliner $\mathrm{MgO}(001)$-Tunnelbarriere und Fe-Elektroden $\ldots \ldots \ldots \ldots \ldots$

2.2. Typische Schichtsysteme . . . . . . . . . . . . . . . 12

2.2.1. Aufbau realer Elemente mit $\mathrm{MgO}$-Barriere und CoFeB-Elektrode . . . . . . . . . . . . . . 14

2.2.2. Herstellungsbedingte Einflüsse auf den TMR . . . . . . 16 2.2.2.1. Kristallisation an der Deckschicht . . . . . . . . 17 2.2 .2 .2 . Abhängigkeit der Bor-Diffusion vom Deckschicht-Material . . . . . . . . . . 17

2.2.2.3. Einflüsse der unterschiedlichen Herstellungsmethoden von MgO-Barrieren . . . . . . . . . 18

2.2.2.4. Diffusion weiterer Elemente . . . . . . . . . 20

3. Experimentelle Methoden 21

3.1. Hochauflösende Transmissionselektronenmikroskopie. . . . . . . 22

3.1.1. Aberrationen . . . . . . . . . . . . . . 23

3.1.2. Aberrationskorrigierte Elektronenmikroskopie und ihre Anwendung. . . . . . . . . . . . . 26

3.1.2.1. Abbildung mit negativer sphärischer Aberration 29

3.1.3. Objektwellenrekonstruktion . . . . . . . . . 30

3.2. Analytische Transmissionselektronenmikroskopie . . . . . . . . . 32

3.2.1. Energiedispersive Röntgenspektroskopie. . . . . . . . . . 32 
3.2.2. Elektronenenergieverlustspektroskopie . . . . . . . . 33

3.2.3. Vor- und Nachteile von EELS gegenüber EDX . . . . . . 37

3.3. IDISMA $\ldots \ldots \ldots \ldots \ldots \ldots$

3.3.1. Driftkorrektur, Mittelung und Bestimmung von Simulationsparametern. ................. 40

3.3.2. Multislice-Simulation von HRTEM-Abbildungen . . . . . 44

3.3.3. Die „Averaged-Projected-Potential“-Näherung . . . . . . 45

3.3.4. Die Verteilungsfunktion für die $\mathrm{c}-\mathrm{MgO} / \mathrm{a}-\mathrm{CoFeB}$ Grenzfläche ................. 46

3.3.5. Vergleich experimenteller und simulierter Abbildungen . 50

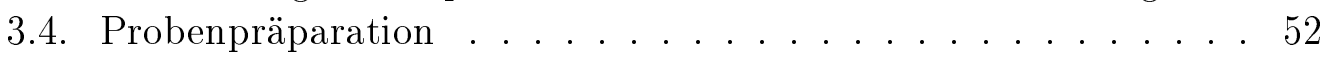

4. Ergebnisse und Diskussion $\quad 57$

4.1. Strukturelle Eigenschaften der $\mathrm{MgO} / \mathrm{CoFe}(\mathrm{B})$-Grenzfläche . . . 57

4.1.1. Die c-MgO/c-CoFe-Grenzfläche . . . . . . . . 58

4.1.1.1. Bestimmung des Gitterabstands senkrecht zur

Grenzfläche ............... 61

4.1.1.2. Sauerstoffversatz an der Grenzfläche . . . . . . 63

4.1.1.3. Zusammenfassende Diskussion der Ergebnisse

\begin{tabular}{|c|}
\hline Zur \\
\hline Untersuchung an der c-MgO/c-CoFe-Grenzfläche 65
\end{tabular}

4.1.2. Struktureller Übergang der c-MgO/a-CoFeB-Grenzfläche 67

4.1.2.1. Iterative Anpassung des strukturellen Übergangs an der Grenzfläche . . . . . . . . . . 71

4.1.2.2. Einfluss von Grenzflächeninhomogenitäten . . . 76

4.1.2.3. Iterative Anpassung des strukturellen Übergangs

4.1.2.4. Zusammenfassende Diskussion der Untersuchung des strukturellen Übergangs der c- $\mathrm{MgO} / \mathrm{a}-\mathrm{CoFeB}$ Grenzfläche ............. 86

4.2. Bor-Diffusion . . . . . . . . . . . . . . . 89

4.2.1. Einfluss der Deckschicht auf die Bor-Diffusion und das Kristallisationsverhalten . . . . . . . . . . . . 89

4.2.2. Einfluss der MgO-Depositionsmethode auf die Bor-Dif-

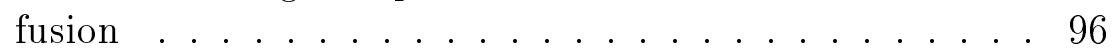

4.2.3. Zusammenfassung der Ergebnisse der Bor-Diffusionsun-



4.3. Zusammenbruch elektrisch gestresster MTJs . . . . . . . . . 103

5. Zusammenfassende Diskussion und Ausblick 109 
\begin{tabular}{ll}
\hline A. Atomares Streupotential & 117
\end{tabular}

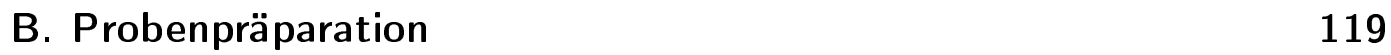

B.1. Herstellung der c-MgO/c-CoFe-Probe . . . . . . . . . . . . . . 120

B.2. Herstellung der c-MgO/a-CoFeB-Probe . . . . . . . . . . . . . 120

B.3. Probeherstellung für die Untersuchung der Deckschichtabhän-

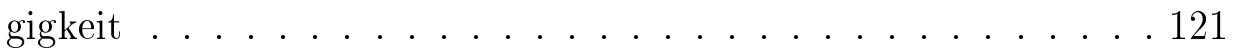

B.4. Herstellung der funktionalen MTJs mit verschiedenen MgO-

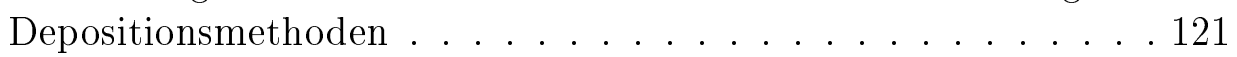

B.5. Herstellung der Proben für die Untersuchung des Durchbruch-

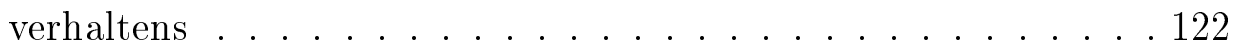

\begin{tabular}{|l|l|l|l|l}
\hline C. Intensitätsprofile und Grenzflächenabbildungen & 123
\end{tabular}

C.1. Profile der Grenzflächenposition 2 . . . . . . . . . . . . . . . 123

C.2. Ergebnisse der iterativen Anpassung mit 10 Parametern. . . . . 126

\begin{tabular}{ll}
\hline Literaturverzeichnis & 131
\end{tabular} 
Inhaltsverzeichnis 


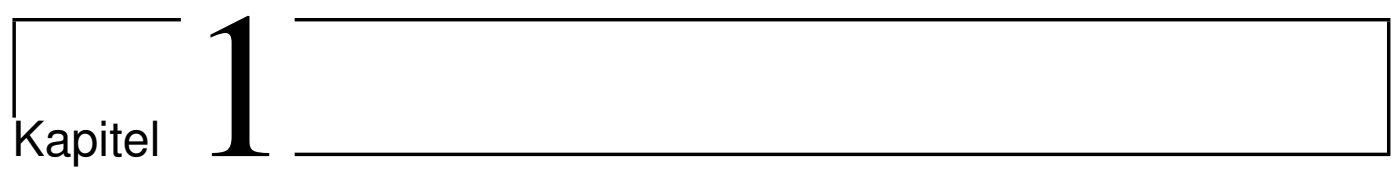

\section{Einleitung}

Seit der Entdeckung des Tunnelmagnetowiderstandeffektes (TMR) durch Jullière im Jahr 1975 [1] haben Tunnelwiderstandselemente (MTJ) eine immense Entwicklung durchgemacht. Jullière konnte experimentell zeigen, dass sich der Widerstand zwischen zwei ferromagnetischen Schichten in Abhängigkeit von deren Magnetisierungsrichtung ändert, wenn diese durch eine dünne isolierende Barriere getrennt werden. Dieser Effekt trat zuerst nur bei tiefen Temperaturen von unter 4,2 K auf und zeigte eine Änderung des Tunnelwiderstandes um nur $14 \%$. Getrieben durch mögliche Einsatzgebiete als Sensorelemente in Leseköpfen von Festplatten [2] oder in magnetischen Speicherelementen (MRAM) 3] wurden diese Tunnelelemente immer weiter optimiert, sodass heutige Systeme bei Raumtemperatur Tunnelmagnetowiderstandswerte (TMR-Werte) von über $600 \%$ [4] aufweisen können. Diese TMR-Steigerung geht im Wesentlichen auf Veränderungen bei den eingesetzten Tunnelbarrieren-Materialien zurück. So nutzte Jullière Ge-O-Barrieren, wohingegen für die ersten RaumtemperaturTMR-Elemente eine amorphe $\mathrm{Al}_{2} \mathrm{O}_{3}$-Barriere verwendet wurde [5, 6].

Spätere Systeme benutzten Tunnelbarrieren aus kristallinem $\mathrm{MgO}$, da diese laut Berechnungen von Butler et al. [7] sowie von Mathon und Umerski [8] deutlich höhere TMR-Werte zeigen sollten. Die Umsetzung in Systemen

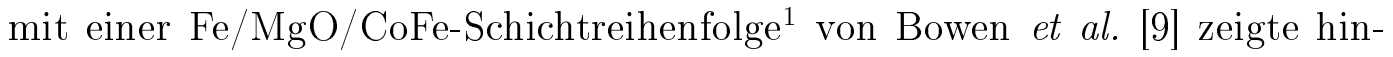
gegen geringere TMR-Werte als bei Systemen mit amorphen $\mathrm{Al}_{2} \mathrm{O}_{3}$-Barrieren. Die Ursache hierfür ist eine schlechte Kopplung der Blochzustände der Elektrode an die der Barriere, welche erst durch eine Auslagerung der Elemente bei hohen Temperaturen erreicht wird. Obwohl inzwischen hohe TMR-Werte in Systemen mit einer MgO-Tunnelbarriere und Elektroden auf Basis einer

${ }^{1}$ Die Schichtreihenfolge $\mathrm{Fe} / \mathrm{MgO} / \mathrm{CoFe}$ gibt an, dass die Elektroden aus Fe und CoFe und die Tunnelbarriere aus $\mathrm{MgO}$ besteht. 
kristallinen Eisen-Kobalt-Legierung erreicht wurden, sind diese Systeme aufgrund ihrer schlechten Integrationsmöglichkeiten in bestehende Prozesse in der Halbleiterindustrie von geringer Bedeutung. Dies änderte sich mit der erfolgreichen Nutzung von amorphen Elektroden, da diese wesentlich einfacher auf andere kristalline Schichten aufgewachsen werden können. Djayaprawira et al. [10] konnten in a-CoFeB/c-MgO/a-CoFeB-Systemen erstmals einen TMR von $230 \%$ bei Raumtemperatur nachweisen 2 . Das Bor in den Elektroden bewirkt beim Aufbringen ein amorphes Wachstum der Elektroden. Durch einen Auslagerungsschritt bei hohen Temperaturen nach der Herstellung der Elemente diffundiert das Bor heraus. Hierdurch wird es den vormals amorphen Elektroden möglich zu kristallisieren und die kristalline MgO-Tunnelbarriere dabei als Kristallisationsgrundlage zu nutzen, sodass es zu einer guten Kopplung der Blochzustände der Elektroden an die Barrierenzustände kommt. Somit kommt der amorph/kristallinen Grenzfläche eine entscheidende Bedeutung zu.

Berechnungen von Heiliger et al. [11] konnten 2007 sogar zeigen, dass bereits wenige Monolagen kristallinen Eisens an der Grenzfläche zum MgO ausreichen sollten, um mit vollständig kristallinen Elektroden vergleichbare TMR-Werte zu erzielen.

Djayaprawira et al. [10, konnten trotz Auslagerung der Elemente in hochauflösenden Transmissionselektronenmikroskopischen Abbildungen (HRTEMAbbildungen) nur vereinzelt kristalline Bereiche in den Elektroden nachweisen. Eine vom kristallinen $\mathrm{MgO}$ ausgehende, in das amorphe Material hineinreichende, induzierte kristalline Ordnung an der Grenzfläche wurde dabei nicht untersucht.

Im Rahmen dieser Arbeit wird die Reichweite der durch das kristalline $\mathrm{MgO}$ induzierten kristallinen Ordnung in amorphem CoFeB in nicht ausgelagerten Systemen quantifiziert, um so eine untere Grenze für diese Reichweite zu erhalten und mit Hilfe von Heiligers Berechnungen, Rückschlüsse auf den TMR ziehen zu können. Eine Methode zur Bestimmung der von einem Material mit Fernordnung in ein Material mit Nahordnung bzw. mittelreichweitiger Ordnung induzierten Ordnung wurde von Borgardt et al. [12, 13, [14, 15] entwickelt und basiert auf einem Vergleich gemittelter, experimenteller und simulierter HRTEM-Abbildungen der Grenzfläche. Diese Methode benutzt zur Beschreibung amorpher Strukturen in Grenzflächennähe eine dreidimensionale Verteilungsfunktion. Die zweidimensionale Projektion dieser Verteilungsfunktion ermöglicht es, HRTEM-Abbildungen mit bereits bekannten Multislice-Simulationsmethoden [16, 17, 18, 19, 20] zu simulieren. Durch Verwendung der „Averaged-Projected-Potential“-Näherung (APP-Näherung)

${ }^{2}$ Trotz bereits erzielter TMR-Werte von $600 \%$ weisen z.Z. „gute“ MTJs auf $\mathrm{CoFeB} / \mathrm{MgO} / \mathrm{CoFeB}-B$ asis üblicherweise TMR-Werte von etwa $200-300 \%$ auf. 
genannten Methode und durch den Vergleich mit experimentellen HRTEMAbbildungen ist es möglich, die atomare Ordnung an der Grenzfläche zu bestimmen.

Im Rahmen dieser Arbeit wird zunächst die für die APP-Näherung notwendige dreidimensionale Verteilungsfunktion angepasst, um die Grenzfläche zwischen kristallinem $\mathrm{MgO}$ und amorphem $\mathrm{CoFeB}$ zu untersuchen. In einem iterativen Prozess wird die Verteilungsfunktion durch einen quantitativen Vergleich von simulierten und experimentellen Abbildungen einer Defokusserie bestimmt.

Das in dieser Arbeit untersuchte Modellsystem besteht aus einer auf einem kristallinen MgO-Substrat nicht ausgelagerten amorphen CoFeB-Schicht. Dieses System weist, im Gegensatz zu den bisher untersuchten a-Ge/c-Si bzw. a-Si/c-Si -Systemen [21, 22], Stufen an der Grenzfläche auf. Deshalb wird die bisher verwendete Methode um die Berücksichtigung dieser Stufen an der Grenzfläche in Strahlrichtung erweitert, wodurch eine um $18 \%$ bessere Anpassung zwischen den simulierten und den experimentellen Abbildungen dieser Probenstelle erreicht werden konnte.

Die angepasste zweidimensionale Verteilungsfunktion zeigt, dass die induzierte Ordnung an der Grenzfläche 0,7 nm in das amorphe CoFeB hineinreicht, was etwa drei bis vier Monolagen kristallinen $\mathrm{CoFe}$ an der Grenzfläche entspricht. Von dieser Schicht ist insbesondere die erste Monolage stark lokalisiert, die nach Heiliger et al., zu einem TMR von maximal 570 \% führen kann [11].

Die Berechnungen von Heiliger setzen voraus, dass das Fe epitaktisch auf dem $\mathrm{MgO}$ aufwächst [11]. Dass CoFeB beim Auslagern vom $\mathrm{MgO}$ aus in einer bcc-Kristallstruktur kristallisiert, ist jedoch nicht selbstverständlich [2, 23 . Außerdem muss hierfür das Bor aus dem CoFeB herausdiffundieren 3 . Berechnungen von Burton zeigten, dass es für Bor energetisch günstiger ist, beim Auslagern an der MgO-CoFe-Grenzfläche zu verbleiben als im kristallisierten CoFe. Diese Segregation von Bor an der Grenzfläche würde aber den TMR, durch eine reduzierte Leitung durch das $\Delta_{1}$-Band, drastisch verringert [24].

Die Kristallisation und insbesondere die Diffusion des Bors beim Auslagern sind bislang schwer zu kontrollierende Prozesse. Neben der Auslagerungszeit und -temperatur [25] beeinflussen auch das Deckschichtmaterial [25, 26, 27, 28] und die Herstellungsweise [28] die Diffusion von Bor in CoFeB-basierenden Systemen.

\footnotetext{
${ }^{3}$ Grundsätzlich werden die Elektroden aller in dieser Arbeit untersuchten Proben aus CoFeB hergestellt. Die Verwendung des Begriffs CoFe soll andeuten, dass es sich um kristallisiertes $\mathrm{CoFeB}$ handelt, aus dem das Bor herausdiffundiert ist; eine begriffliche Unterscheidung ist jedoch nicht überall eindeutig möglich.
} 
In dieser Arbeit wird der Einfluss des Deckschichtmaterials auf die BorDiffusion und die mögliche Kristallisation des CoFeB an der Deckschicht untersucht. Ebenso wird der Einfluss der Herstellungsmethode der isolierenden MgO-Tunnelbarriere auf das Segregationsverhalten von Bor analysiert.

Die Deckschicht soll in diesen Systemen die Kristallisation von CoFeB nicht beeinflussen und darüber hinaus als Senke für Bor dienen.

Für die Untersuchungen in dieser Arbeit werden die beiden Deckschichtmaterialien ausgewählt, die tendenziell die größten TMR aufweisen: Tantal und Ruthenium [29]. Diese erfolgen mittels HRTEM und Elektronenenergieverlustspektroskopie (EELS) an einem Modellsystem in dem auf ein $\mathrm{MgO}-$ Substrat eine CoFeB-Schicht und dann die jeweilige Deckschicht aufgebracht wurde. Es stellte sich heraus, dass Tantal in der Lage ist, als Senke für das herausdiffundierende Bor zu dienen, wohingegen Ruthenium die Bor-Diffusion blockiert. Bei der Kristallisation des CoFeB zeigte sich, dass das nanokristalline Ta keinen Einfluss darauf besitzt. hcp-Ru kann zwar eine Kristallisation des CoFeB von der Deckschicht beeinflussen, bei dünnen CoFeB-Schichten, wie diese üblicherweise in MTJs eingesetzt werden, bildet sich aber eine amorphe Zwischenschicht zwischen $\mathrm{CoFeB}$ und $\mathrm{Ru}$, sodass keine Kristallisation von der Deckschicht aus auftritt. In keiner dieser Modellsystemproben konnte Bor im $\mathrm{MgO}$ gefunden werden.

Da Bor am oder im $\mathrm{MgO}$ in der Literatur in zahlreichen Arbeiten nachgewiesen wird [25, 30, 31, 32, wird der Einfluss der MgO-Barrieren-Herstellungsmethode an funktionsfähigen MTJs untersucht. Hier zeigen die EELS-Spektren eine Kontamination der MgO-Tunnelbarriere mit Boroxid bei Verwendung von Hochfrequenz-gesputterten (kurz rf-gesputtert) MgO-Barrieren, die bei elektronenstrahldeponierten $\mathrm{MgO}-$ Barrieren nicht auftritt. Das Bor im [rf]-MgO ist senkrecht zur Barriere homogen verteilt, es tritt also keine Segregation an den Grenzflächen auf.

Eine mögliche Ursache für die Segregation von Bor in der [rf]-gesputterten MgO-Tunnelbarriere ist eine Diffusion von Bor entlang von Korngrenzen der texturierten $\mathrm{MgO}$-Barriere [33]. Komagaki et al. [33] vermuten, dass diese Borkontaminierten Korngrenzen ,pinholes“ in der Tunnelbarriere bilden. Bei Thomas et al. [34] führen diese ,pinholes“ unter elektrischer Überlastung zu einem Bruch der Barriere [34].

Der letzte Teil dieser Arbeit widmet sich Untersuchungen an elektrisch überlasteten und gestressten Tunnelelementen. Diese wurden mittels Spannungspulsen unterschiedlicher Dauer belastet und vor und nach der elektrischen Belastung der TMR gemessen. Die gestressten Elemente weisen nach der Belastung noch einen geringen Rest-TMR auf, was im Vergleich zu intakten und völlig zerstörten TMR-Elementen mögliche Stadien des Zusammenbruchs 
sichtbar macht.

Im Einzelnen gliedert sich diese Arbeit in fünf Kapitel: Kapitel 2 gibt eine Einführung in das Themengebiet des TMR-Effekts und den Aufbau typischer Schichtsysteme. Dabei werden die herstellungsbedingten Einflüsse auf den TMR in diesen Systemen genauer betrachtet. Das Kapitel 3 gibt eine kurze Einführung in die in dieser Arbeit benutzten experimentellen Methoden. Schwerpunkte bilden dabei die Darstellung der genutzten APP-Näherung sowie die für diese Arbeit erforderlichen Modifikationen bei der Konstruktion der dreidimensionalen Verteilungsfunktion. Kapitel 4 zeigt die Ergebnisse der Untersuchung der amorph/kristallinen Grenzflächen von CoFeB/MgO-Systemen sowie Ergebnisse aus der Untersuchung der Bor-Diffusion und an überspannungszerstörten Tunnelelementen. Im abschließenden Kapitel 5 werden alle Ergebnisse zusammengefasst und diskutiert und ein Ausblick auf mögliche weitere Untersuchungen gegeben. 
1. Einleitung 


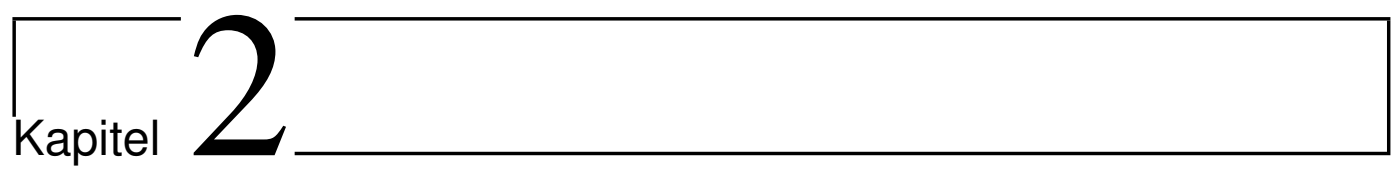

\section{Einführung}

\subsection{TMR-Effekt}

Im Folgenden werden die für das Verständnis der vorliegenden Arbeit wesentlichen Eigenschaften des Tunnelmagnetowiderstands behandelt. Einen Überlick über den Themenkomplex bietet u.a. die Arbeit von Yuasa et al. [2, 35]; eine weitergehende theoretische Betrachtung des Tunnelprozesses ist außerdem bei Butler et al. [36] zu finden.

Der Tunnelmagnetowiderstand ist seit seiner Entdeckung 1975 von Jullière [1] Gegenstand intensiver Forschung. Seitdem der magnetische Tunnelwiderstand bei Raumtemperatur nachgewiesen werden kann [5, 6], ist dieser Effekt auch aufgrund zahlreicher Anwendungen technologisch interessant.

MTJ bestehen im Wesentlichen aus einer dünnen isolierenden Tunnelbarriere und zwei ferromagnetischen Elektroden (s. Abb. 2.1). Wird eine Spannung senkrecht zur Tunnelbarriere angelegt, so müssen die Elektronen diesen Isolator durch Tunneln überwinden. Der Tunnelwiderstand ist dabei abhängig von der relativen Magnetisierung der ferromagnetischen Elektroden. Ist die Magnetisierung der beiden Elektroden parallel zueinander, ist der Widerstand in der Regel geringer als bei antiparalleler Magnetisierung (s. Abb. 2.1). Als Tunnelmagnetowiderstandswert wird die normierte Widerstandsdifferenz

$$
T M R=\frac{R_{a p}-R_{p}}{R_{a p}}
$$

bezeichnet. $R_{a p}$ bezeichnet den Widerstand bei antiparalleler und $R_{p}$ den Widerstand bei paralleler Magnetisierung. Die Tunnelwiderstandselemente von Jullière [1] bestanden aus einem $\mathrm{Fe} / \mathrm{Ge}-\mathrm{O} / \mathrm{Co}$-System und wiesen bei 4,2 K 
einen TMR-Wert von $14 \%$ auf, welcher bei späteren Arbeiten kontinuierlich verbessert wurde. So wiesen die ersten Raumtemperatur-Ergebnisse an TMRElementen mit amorpher $\mathrm{Al}_{2} \mathrm{O}_{3}$-Tunnelbarriere [5, 6] bereits TMR-Verhältnisse von bis zu $18 \%$ auf. Die Entwicklung machte 2004 durch die Verwendung kristalliner Tunnelbarrieren einen weiteren großen Sprung. Bei Yuasa et al. [37] konnte dadurch der TMR bei Raumtemperatur auf $88 \%$ für Fe/MgO/FeSysteme erhöht werden. Zur Zeit liegen die maximal gemessenen TMR bei ca.

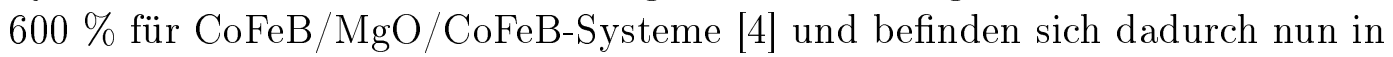
einem Bereich von über $150 \%$, in dem sie für praktische Anwendungen nutzbar sind [2].
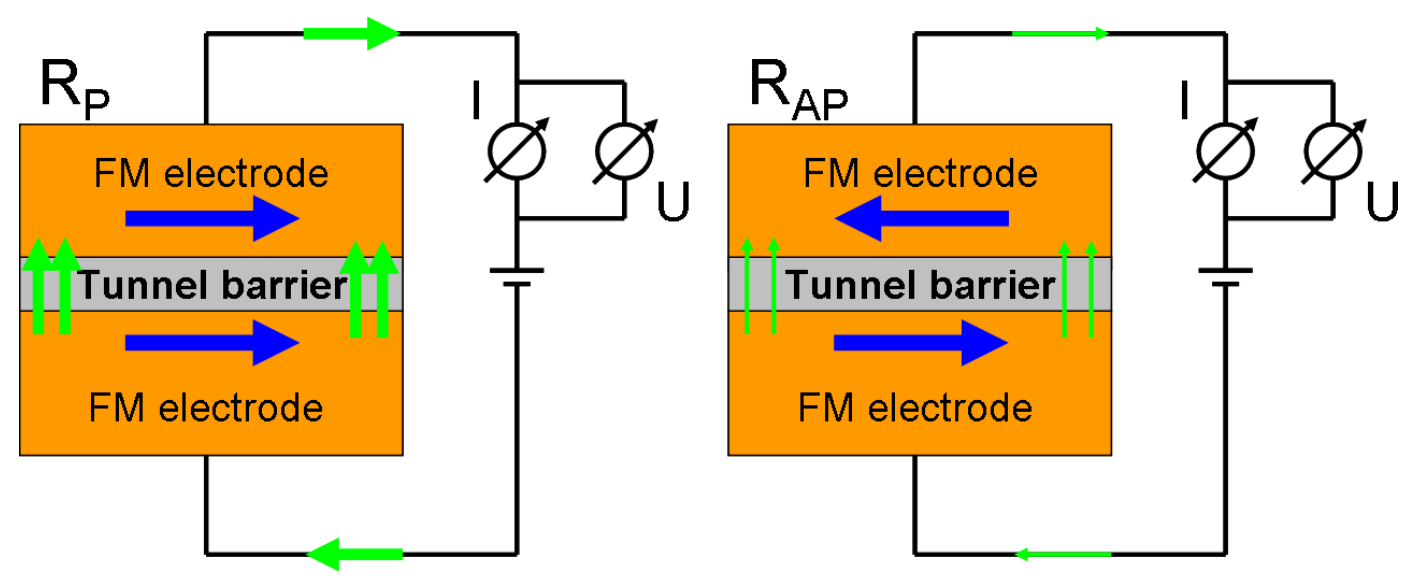

Abbildung 2.1.: Schematische Darstellung des TMR-Effektes in einem MTJ. Geringer Tunnelwiderstand im Fall paralleler Magnetisierung (links) und hoher Tunnelwiderstand bei antiparalleler Magnetisierung. Nach [2].

\subsubsection{Spinpolarisation}

Einfache Modellsysteme für das Auftreten des TMR-Effektes stützen sich ausschließlich auf die Spinpolarisation der tunnelnden Elektronen. Als entscheidend gilt, dass der Elektronenspin während des Tunnelns durch die Barriere erhalten bleibt.

Mittels Magnetisierung der Elektroden wird der Spin eines Großteils der Elektronen parallel zur Magnetfeldrichtung ausgerichtet. Die so spinpolarisierten Elektronen bezeichnet man als Majoritätsspinelektronen im Gegensatz zu den Minoritätsspinelektronen, die entgegengesetzt polarisiert sind. Ist nun die Magnetisierung der beiden Elektroden gleichgerichtet, bleibt auch der Spin der tunnelnden Elektronen gleich. Damit die Elektronen durch die Barriere tunneln können, müssen auch in der zweiten Elektrode genügend unbesetzte 
Zustände vorhanden sein. Dabei spielen in metallischen Elektroden hauptsächlich Zustände nahe der Fermi-Energie eine Rolle. Im Falle einer parallelen Elektrodenausrichtung stehen in der zweiten Elektrode ausreichend unbesetzte Zustände für die Minoritätsspinelektronen zur Verfügung, da sie an der Fermi-Kante eine höhere Zustandsdichte haben als die Majoritätsspinelektronen. Deshalb können von den Minoritätsspinelektronen mehr Elektronen zum Tunnelstrom beitragen.

Ist die Magnetisierung der Elektroden jedoch antiparallel, werden beim Tunneln die Majoritäts- zu Minoritätsspinelektronen und umgekehrt. Jedoch stehen in diesem Fall nur wenige freie Zustände für Minoritätsspinelektronen in der zweiten Elektrode zur Verfügung, was zu einem geringeren Tunnelstrom führt (s. Abb. 2.2) 2].


Abbildung 2.2.: TMR-Effekt nach Jullière: Zustandsdichte $D$ bei FermiEnergie $E_{F}$ in Elektrode 1 und 2 für die Majoritätsspinelektronen $\uparrow$ und Minoritätsspinelektronen $\downarrow$. Aus [2].

\subsection{2. $T M R$ in $M T J$ mit kristalliner $\mathrm{MgO}(001)-T u n n e l b a r r i e r e$ und Fe-Elektroden}

Ein Sonderfall des Tunnelvorgangs tritt auf, wenn die tunnelnden Elektronen in der Barriere nicht gestreut werden und sich der Wellenvektor der Elektronen somit nicht ändert. Eine Streuung tritt bei amorphen Barrieren auf Grund von Überlagerungen inkohärenter Vorgänge auf. Bleibt der Wellenvektor der Elektronen unverändert, was in kristallinen Barrieren der Fall ist, so spricht man von kohärentem Tunneln [2, 36].

Ein vollständig kristallines System aus $\mathrm{Fe}(001) / \mathrm{MgO}(001) / \mathrm{Fe}(001)$ lässt sich deshalb nicht mit dem einfachen Jullière-Modell beschreiben. Mavropoulos et al. [38 beschrieben zuerst ein Modell, in dem die Kopplung der Bloch-Zustände 
in der ferromagnetischen Elektrode und die abklingenden Zustände in der Barriere einen entscheidenen Einfluss haben. Weitergehende Berechnungen zu diesem System wurden anschließend auch von Butler et al. [36] durchgeführt. In Abbildung 2.3 sind die berechneten Energiebänder für bcc-Eisen parallel zur Grenzfläche für die Majoritäts- und Minoritätsspinelektronen dargestellt. Es ist ersichtlich, dass das $\Delta_{1}$-Band freie Zustände an der Fermi-Energie nur für Majoritätsspinelektronen aufweist sowie $\Delta_{2}$-Zustände nur für Minoritätsspinelektronen. Die Bänder $\Delta_{5}$ und $\Delta_{2^{\prime}}$ weisen für beide Spinrichtungen mögliche Zustände an der Fermi-Energie auf.
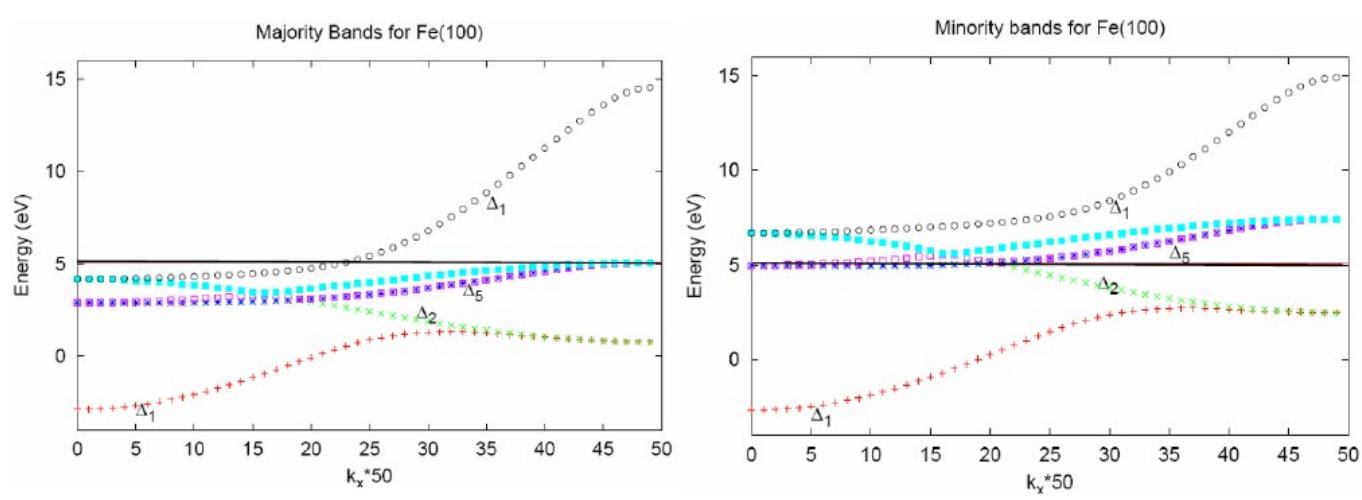

Abbildung 2.3.: Bandstruktur für bcc-Eisen parallel zur Grenzfläche für die Majoritätsspinelektronen (links) und Minoritätsspinelektronen (rechts). Die durchgezogene Linie kennzeichnet die Fermi-Energie. Aus [36].

Betrachtet man nun die Tunnelzustandsdichten in Fe/MgO/Fe-Systemen (s. Abb. 2.4), fällt auf, dass die von links aus der Elektrode einlaufenden BlochZustände, die in der Barriere in der Bandlücke liegen, dort exponentiell abklingen. Bei paralleler magnetischer Ausrichtung der Elektroden erkennt man in den Tunnelzustandsdichten, dass die $\Delta_{2^{\prime}}$-Zustände sehr viel schneller in der Barriere abklingen, als die $\Delta_{5}$-Zustände. Im Minoritätsspinkanal finden sich noch $\Delta_{2}$-Zustände, die im Majoritätsspinkanal nicht vorhanden sind, aber in ihrer Abklingrate zwischen denen der $\Delta_{5^{-}}$und $\Delta_{2^{\prime}}$-Zustände liegen. Sehr viel geringere Abklingraten im $\mathrm{MgO}$ weisen die $\Delta_{1}$-Zustände im Majoritätsspinkanal auf, deren Zustände im Minoritätsspinkanal nicht vorhanden sind. Somit wird deutlich, dass bei paralleler Magnetisierungsrichtung der Elektroden die Elektronen mit $\Delta_{1}$-Symmetrie den größten Beitrag zum Tunnelstrom leisten. Bei antiparalleler Magnetisierung sind wieder in beiden Spinkanälen die sehr kurzlebigen (stark abklingenden) $\Delta_{2^{\prime}}$ und $\Delta_{5}$ Symmetrien vorhanden. Die $\Delta_{2^{-}}$ Zustände der Minoritätsspinelektronen klingen zwar in der Barriere nicht so stark ab wie die Zustände mit $\Delta_{2^{\prime}}$ Symmetrie, finden jedoch in der rechten 
Elektrode keine besetzbaren Zustände, da sie hier Majoritätsspinelektronen sind und deshalb vollständig reflektiert werden. Ähnlich verhält es sich mit Zuständen mit $\Delta_{1}$-Symmetrie im Majoritätsspinkanal. Diese klingen in der Barriere nur schwach ab, finden jedoch auch keine besetzbaren Zustände in der rechten Elektrode vor.

Aus dieser Eigenschaft als Symmetriefilter wird deutlich, dass dem $\Delta_{1}$-Band für den Tunnelprozess in Fe/MgO/Fe-MTJ eine entscheidende Bedeutung zukommt und es maßgeblich für die hohen TMR in diesen Systemen verantwortlich ist [36].
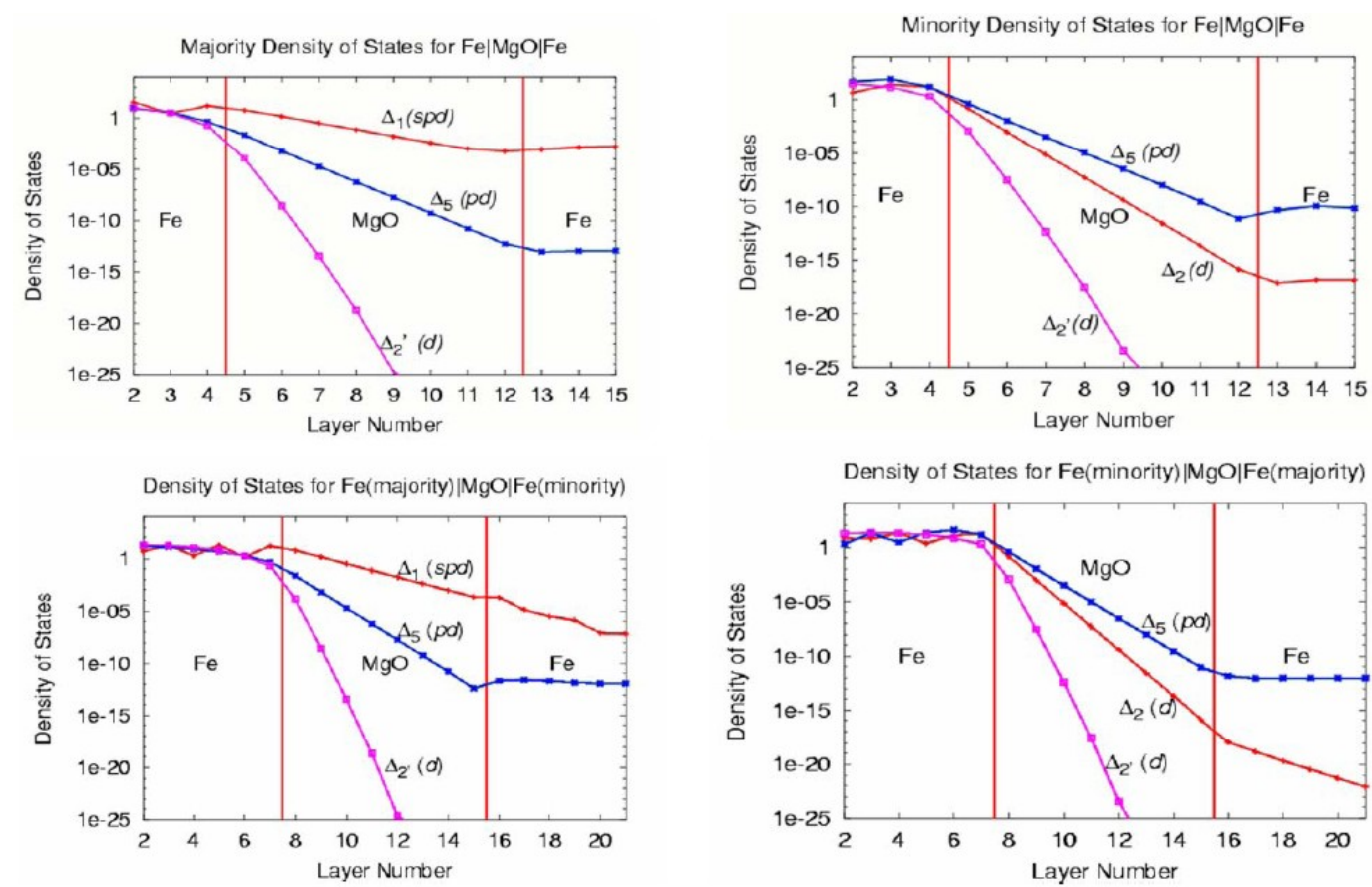

Abbildung 2.4.: Tunnelzustandsdichte für $\mathrm{Fe} / \mathrm{MgO} / \mathrm{Fe}$ in paralleler (oben) und antiparalleler (unten) magnetischer Orientierung der Elektroden jeweils für Majoritätsspins (links) und Minoritätsspins (rechts). Aus [36].

Ausgehend von diesen Erkenntnissen, dehnten Zhang und Butler 2004 [39] ihre Berechnungen auf Systeme mit bcc-Co(100) und $\mathrm{CoFe}(100)$ als Elektrodenmaterial aus. Es zeigte sich, dass im Co nur Majoritätsspinelektronen mit $\Delta_{1}$-Symmetrie die Fermi-Energie schneiden und nicht wie bei Fe noch das $\Delta_{2^{\prime}}$ und $\Delta_{5}$-Band. Dies führt zu einer Voraussage noch viel höher zu erzielender TMR in diesen Systemen, weil nur das Band mit Symmetriefiltereffekt eine Rolle spielt. 


\subsection{Typische Schichtsysteme}

Wie aus dem vorangegangenen Abschnitt ersichtlich, basieren $\mathrm{Fe} / \mathrm{MgO} / \mathrm{Fe}-$ Systeme auf epitaktisch gewachsenen Systemen mit kristalliner Elektrode und kristalliner MgO-Barriere.

$\mathrm{MgO}$ selbst liegt in einem Ionengitter mit NaCl-Struktur vor, welches als kubisch-flächenzentriertes (fcc)-Gitter mit zweiatomiger Basis beschrieben werden kann. Die Gitterkonstante von $\mathrm{MgO}$ beträgt 0,421 nm [40, 41].

In Untersuchungen wurde gezeigt, dass $\mathrm{MgO}(100)$ auf Eisen(001) pseudomorph aufwächst [42]. Dabei sind die Einheitszellen von $\mathrm{MgO}$ und Fe um $45^{\circ}$ zueinander verdreht, sodass die Mg0[110]-Richtung parallel zur Fe[010]Richtung liegt (s. Abb. 2.5). Aus der Gitterfehlpassung von 3,5\% kommt es zu einer kompressiven Verspannung des $\mathrm{MgO}$, welche ab etwa sieben Monolagen $\mathrm{MgO}$ durch Versetzungen relaxiert [42].
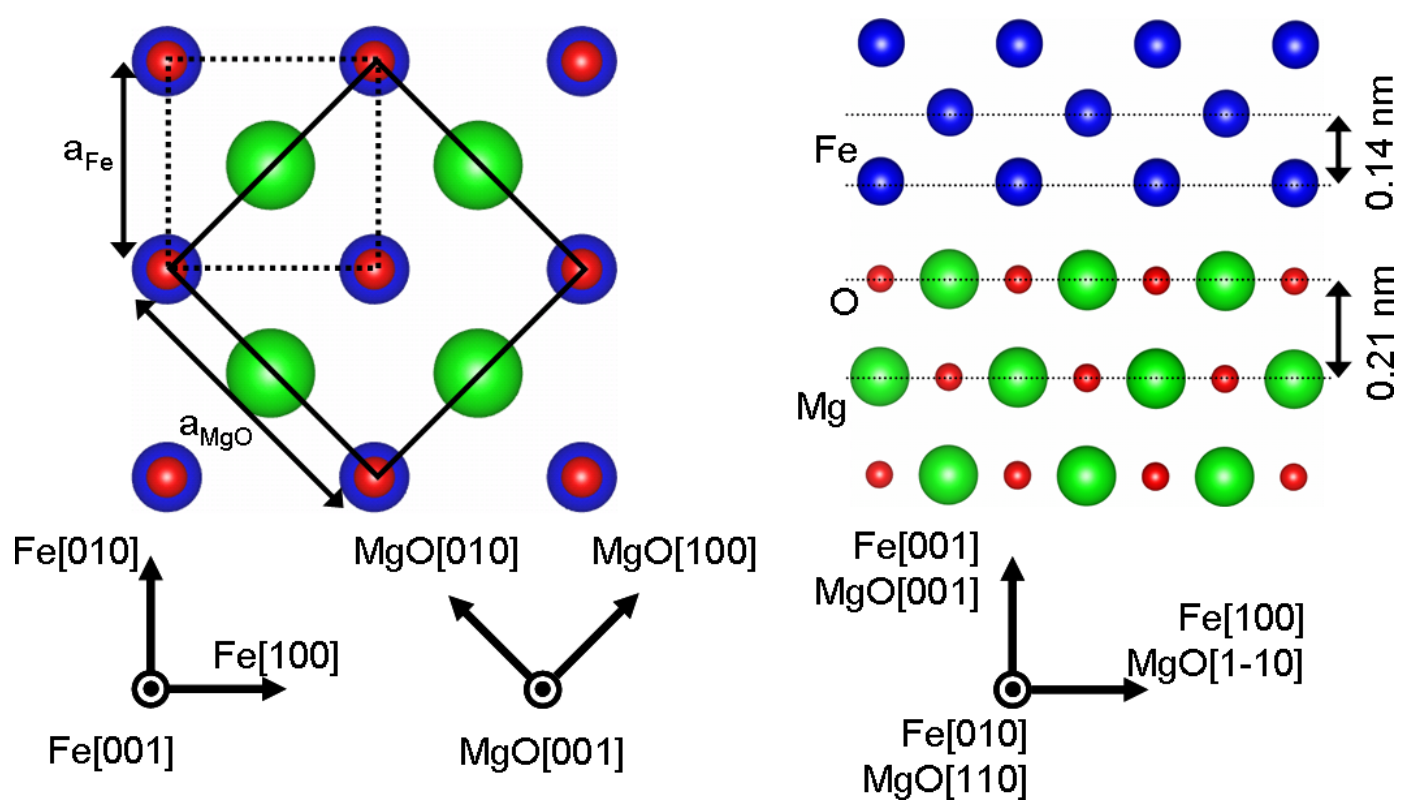

Abbildung 2.5.: Pseudomorphes Wachstum von $\mathrm{MgO}(001)$ und bcc-Fe(001). Durch eine Drehung der Einheitszellen um $45^{\circ}$ wird die geringste Gitterfehlpassung erreicht. Dadurch liegen $\mathrm{Fe}[010]$ und $\mathrm{MgO}[110]$ an der Grenzfläche parallel zueinander. Nach [2].

Technisch ist dieses Wachstum durchaus anspruchsvoll, da sich $\mathrm{MgO}$ auf Fe lagenweise aus der Gasphase abscheidet (Frank-van-der-Merve-Wachstum), worauf das Fe der zweiten Elektrode bei Raumtemperatur zuerst inselartig wächst (Volmer-Weber-Wachstum) [43]. 
Dieses aus einigen Monolagen kristallinem MgO und epitaktisch gewachsenen, ausgedehnten Fe-Elektroden bestehende Materialsystem wurde auch von Butler et al. [7] und Mathon und Umerski [8] anhand von TMR-Berechnungen untersucht. Es zeigte sich, dass durch den epitaktischen Übergang zwischen $\mathrm{MgO}$ und Fe theoretisch ein hoher TMR von über $1000 \%$ erreicht werden kann.

Djayaprawira et al. [10] stellten 2005 als Erste ein Tunnelelement her, welches amorphe CoFeB-Elektroden um eine kristalline MgO-Barriere herum aufwies. Die Nutzung amorpher Elektroden ist in prozesstechnologischer Hinsicht interessant (s. Abschnitt 2.2.1). Überraschenderweise zeigte dieses Element bei Raumtemperatur einen erstaunlich hohen TMR von 230\%. Eine Erklärung konnte hierzu nicht gegeben werden. Man vermutete, dass der Einfluss der Grenzflächenrauheit einen Einfluss auf den TMR hat. Aufgrund der gegebenen HRTEM-Abbildungen konnte aber auch nicht geklärt werden, ob einige Monolagen $\mathrm{CoFeB}$ an der Grenzfläche zum MgO kristallisiert waren. 2007 gab es durch neue Berechnungen von Heiliger und Gradhand et al. [11, 44] eine Erklärung. Sie berechneten den TMR für amorphe Eisen-Elektroden mit verschieden Monolagen kristallinem bcc-Fe an der Grenzfläche zum MgO (s. Abb. 2.6.

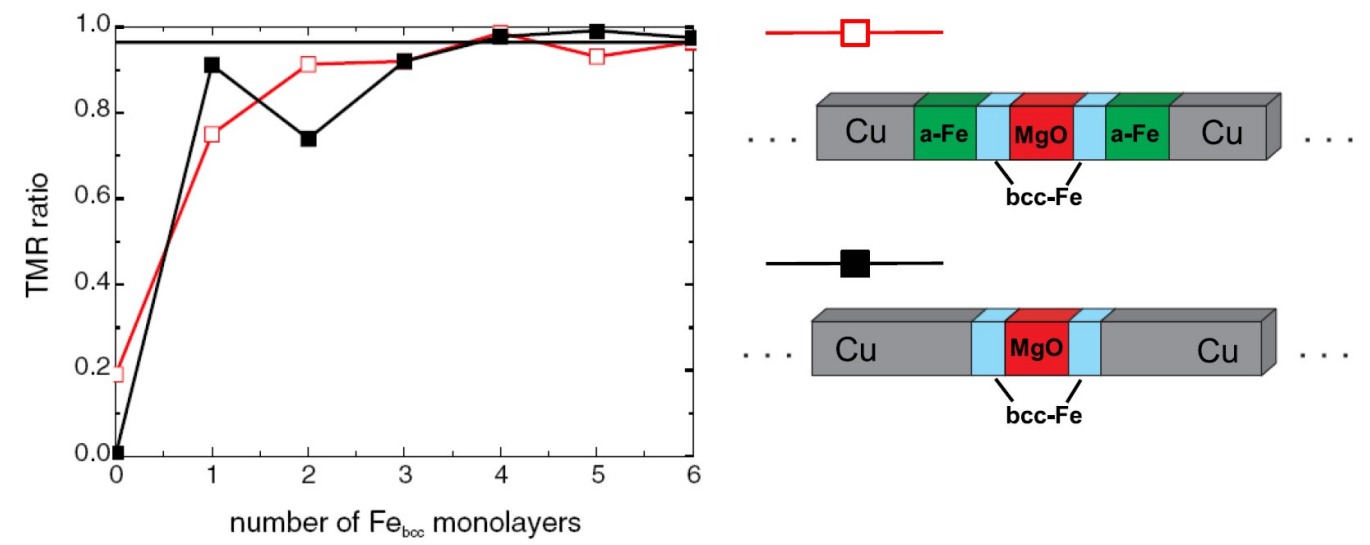

Abbildung 2.6.: Normierte TMR in Abhängigkeit der Anzahl kristalliner Monolagen bcc-Fe an der Grenzfläche zum MgO mit daran anschließendem amorphem Eisen (rot) oder Kupfer (schwarz). Die durchgezogene schwarze Linie repräsentiert den TMR für ausgedehnte kristalline Eisenelektroden. Entnommen aus [45] nach [11].

Dabei zeigte sich, dass für vollständig amorphe Elektroden nur geringe TMR von etwa $44 \%$ zu erwarten sind. Jedoch würde bereits eine Monolage bcc-Fe an der Grenzfläche zum MgO reichen, um einen hohen TMR von 570 \% zu erzielen. Schon ab etwa vier Monolagen kristallinen Eisens zeigt sich, dass zwischen 
diesen Systemen und ausgedehnten kristallinen Elektroden kein signifikanter Unterschied mehr besteht.

Diese wenigen Monolagen kristallinen Materials, die es bedarf, um hohe TMR zu erzielen, können durch eine Auslagerung der Proben bei Temperaturen von über $250{ }^{\circ} \mathrm{C}$ nach dem Wachstum ausgebildet werden.

Djayaprawira et al. wählten als Elektrodenmaterial eine $\mathrm{Co}_{60} \mathrm{Fe}_{20} \mathrm{~B}_{20}$-Legierung. Das Bor dient während des Wachstums bei Raumtemperatur ausschließlich zur Stabilisierung der amorphen Struktur. Aufgrund seiner geringen Größe diffundiert das Bor bei höheren Temperaturen aus den Elektroden heraus und sorgt dafür, dass amorphes CoFeB in Nachbarschaft zu MgO in einer bcc(001)Struktur kristallisiert [23, 46], obwohl die stabile Struktur von CoFe eigentlich eine fcc-Struktur ist. Das CoFe nutzt dabei das $\mathrm{MgO}$ als Kristallisationsvorlage [2].

Andersherum ist texturiertes Wachstum von $\mathrm{MgO}$ auf amorphen Schichten jedoch sehr ungewöhnlich. So wächst $\mathrm{MgO}$ auf $\mathrm{SiO}_{2}$ bei Raumtemperatur auf den ersten 3-4 nm amorph auf. In situ RHEED-Untersuchungen (Reflective High-Energy Electron Diffraction) des Wachstumsprozesses von $\mathrm{MgO}$ auf amorphem CoFeB zeigten, dass das $\mathrm{MgO}$ bis zu einer Dicke von $1 \mathrm{~nm}$ amorph aufwächst und erst danach entlang der [001]-Richtung texturiert kristallisiert. Nach Abschluss des Wachstums zeigt sich die ganze MgO-Schicht kristallisiert [23, 46, 47].

Die Elektroden von Djayaprawira et al. [10] wiesen trotz Auslagerung keine sichtbare, großflächige, kristalline Ordnung auf. Aufgrund der gezeigten hohen TMR in diesem System kann evtl. die langreichweitige Ordnung an der Grenzfläche für eine ausreichende kristalline Ordnung im CoFeB zum MgO ausreichen, um erhöhte TMR zu erzielen.

\subsubsection{Aufbau realer Elemente mit MgO-Barriere und CoFeB-Elektrode}

Wie oben bereits erwähnt, ist die Nutzung amorpher Elektroden insbesondere in prozesstechnologischer Hinsicht interessant. Es können zum einen die amorphen Elektroden und die MgO-Tunnelbarrieren in einem Sputterprozess hergestellt werden. Zum anderen können die so entstandenen Tunnelelemente sehr gut mit anderen Schichten unterschiedlicher Kristallstruktur kombiniert werden, da die amorphe Elektrode als Pufferschicht dient. Für eine praktische Anwendung als MRAM oder Lesekopf in Festplatten ist es erforderlich, die Magnetisierungsrichtung der Elektroden gezielt zu beeinflussen. Hierfür wird in der Regel die untere ferromagnetische Elektrode durch eine ,synthetische ferrimagnetische Struktur" (Synthetic ferrimagnetic structure, SyF) in eine mag- 
netische Richtung fixiert (s. Abb. 2.7). Die obere ferromagnetische Elektrode bleibt frei magnetisierbar, z.B. durch ein äußeres magnetisches Feld [30]. Die synthetische ferrimagnetische Struktur ist eine antiferromagnetisch gekoppelte Schichtstruktur, bestehend aus einer ferromagnetischen, einer nichtmagnetischen und einer weiteren ferromagnetischen Schicht. In der Regel handelt es sich bei der untersten Schicht um CoFe, bei der nichtmagnetischen z.B. um Ruthenium und bei der oberen ferromagnetischen Schicht um die untere Elektrode aus CoFeB. Unter der SyF befindet sich eine weitere antiferromagnetische Schicht (typischerweise PtMn oder IrMn), welche die Magnetisierung der darüberliegenden ferromagnetischen Schicht in eine Richtung festlegt [30]. Diese synthetische ferrimagnetische Struktur ist in anderen Anwendungen bereits erprobt und zeichnet sich durch ein geringes magnetisches Streufeld aus, welches die obere Elektrode nicht beeinflusst [2]. Der Nachteil liegt jedoch darin, dass diese Struktur eine fcc-Struktur mit (111)-Orientierung und einer dreizähligen in-plan-Symmetrie aufweist, die nicht mit der vierzähligen Kochsalzstruktur der MgO-Tunnelbarriere kompatibel ist. Hier wird deutlich, dass der Verwendung einer amorphen Elektrode eine entscheidende Bedeutung auf dem Weg zur technologischen Anwendung zukommt, da hiermit die MgO-Tunnelbarriere problemlos auf die synthetische ferrimagnetische Struktur aufgewachsen werden kann.

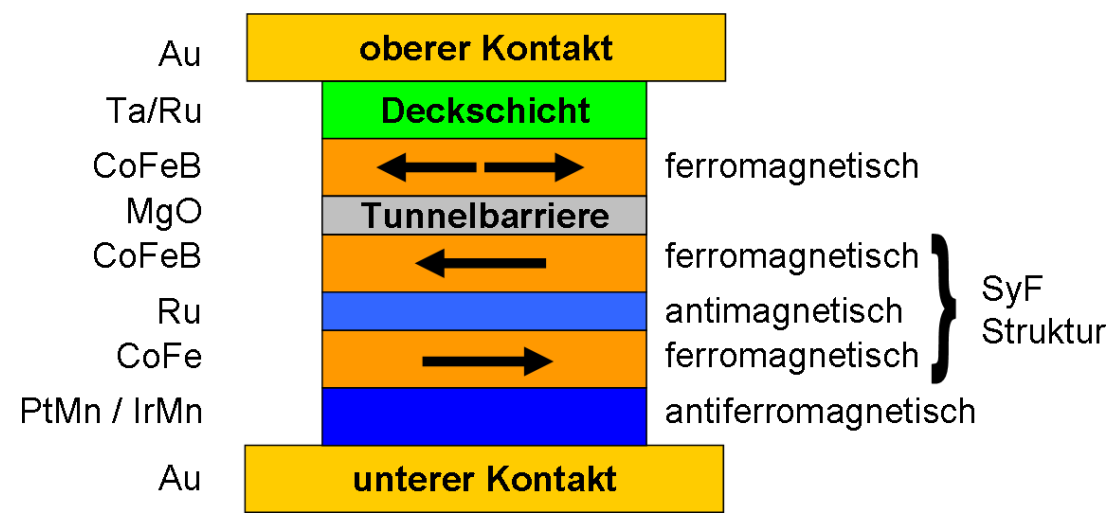

Abbildung 2.7.: Schematischer Querschnitt durch ein Magnetotunnelelement für praktische Anwendungen.

Oberhalb der oberen Elektrode befindet sich die Deckschicht, die als Pufferschicht zum abschließenden (Gold-)Kontakt dient. Die Rolle der Deckschicht wird in den Abschnitten 2.2.2.1 und 2.2.2.2 noch näher betrachtet. 


\subsubsection{Herstellungsbedingte Einflüsse auf den TMR}

Im Folgenden werden drei wichtige Effekte diskutiert, von denen bekannt ist, dass sie einen Einfluss auf den TMR in $\mathrm{CoFeB} / \mathrm{MgO} / \mathrm{CoFeB}-\mathrm{MTJ}$ aufweisen. Dabei hat die Schicht auf der $\mathrm{MgO}$ gegenüberliegenden Seite des CoFeB in zweierlei Hinsicht einen Effekt: So soll diese Schicht die Kristallisation des CoFeB beim Auslagern nicht beeinflussen (s. Abschnitt 2.2.2.1). Darüber hinaus besteht auch eine Abhängigkeit der Bordiffusion von dieser Schicht (s. Abschnitt 2.2.2.2). Häufig wird für die Deckschicht Ruthenium oder Tantal eingesetzt, da MTJs mit diesen Deckschichten die höchsten TMR aufweisen (s. Abb. 2.8).



Abbildung 2.8.: TMR bei Raumtemperatur für verschiedene DeckschichtMaterialien für CoFeB $/ \mathrm{MgO} / \mathrm{CoFeB}$-Systeme. Aus [29].

Neben der Deckschicht konnten Studien zeigen, dass auch die Herstellungsweise der MgO-Tunnelbarriere einen Einfluss auf die Bordiffusion und damit auf den TMR aufweist (s. Abschnitt 2.2.2.3] [28]. Bei dieser Betrachtung ist zu beachten, dass die genannten Prozesse nicht unabhängig von einander sind. Ferner kann auch die Auslagerungstemperatur nicht unabhängig vom Deckschichtmaterial betrachtet werden. Eine umfangreiche Studie zum Einfluss der Auslagerungstemperatur auf verschieden Deckschichtmaterialien findet sich bei Karthik et al. [25]. 


\subsubsection{Kristallisation an der Deckschicht}

Beim Auslagern der MTJs kann das CoFeB nicht nur an der Grenzfläche zum MgO kristallisieren, sondern auch an der Grenzfläche zur Deckschicht. Diese Deckschicht sollte im besten Fall die Kristallisation des CoFeB am MgO nicht beeinflussen, sodass ein kohärentes Tunneln durch das $\mathrm{MgO}$ gegeben ist. Besonders eignen sich deshalb amorphe oder nanokristalline Materialien als Deckschicht.

Die Störung der Kristallisation der oberen CoFeB-Elektrode lässt sich gut anhand von $\mathrm{Ni}_{0.8} \mathrm{Fe}_{0.2}$ erkennen. Auf amorphes $\mathrm{CoFeB}$ gewachsen, kristallisiert $\mathrm{NiFe}$ in eine fcc(111)-Struktur [26]. Beim Auslagern startet von der Deckschicht aus die Kristallisation von CoFeB in eine fcc(111)-Struktur schon bei Temperaturen um $200{ }^{\circ} \mathrm{C}$. Erst ab $250^{\circ} \mathrm{C}$ startet dann die Kristallisation vom $\mathrm{MgO}$ aus. Folglich weist die Elektrode keine bcc(001)-Struktur auf, welche für ein kohärentes Tunneln durch die Barriere erforderlich ist. Das führt zu einer signifikanten Reduktion des TMR.

Bei der Verwendung von Ta als Deckschicht ist entscheidend, auf welchem Material es aufgebracht wird. So zeigt auf kristallines Ru gewachsenes Ta selbst eine kristalline Struktur, die nicht vorhanden ist, wenn das Ta auf ein amorphes Material, wie z.B. a-CoFeB oder a-Si, gewachsen wurde [25].

Untersuchungen von Karthik et al. [28] zeigen für Auslagerungstemperaturen von $300{ }^{\circ} \mathrm{C}$ und $400{ }^{\circ} \mathrm{C}$ bei Verwendung von hcp-Ti und hcp-Ta als Deckmaterial keinen signifikanten Einfluss auf die Kristallisation des CoFeB. In beiden Fällen weist das $\mathrm{CoFe}$ eine bcc(001)-Orientierung auf, wohingegen bei der Verwendung von fcc(111)-Pd als Deckmaterial das CoFe eine bcc(110)-Orientierung aufweist. Bei höheren Temperaturen von $500{ }^{\circ} \mathrm{C}$ oxidieren das hcp-Ti und das hcp-Ta unter Bildung einer amorphen $\mathrm{Ti}(-\mathrm{B})-\mathrm{O}_{\mathrm{x}^{-}}$- bzw . $\mathrm{Ta}(-\mathrm{B})-\mathrm{O}_{\mathrm{x}}-$ Struktur. Spätestens bei Auslagerungstemperaturen von $650{ }^{\circ} \mathrm{C}$ und der Verwendung einer Ta-Deckschicht verliert jedoch die CoFe-Schicht seine bcc(001)-Kristallstruktur [25].

Der Einfluss einer Ru-Deckschicht auf die CoFeB-Kristallisation wird in der Literatur kontrovers diskutiert. So zeigt eine Untersuchung von Ibusuki et al. [48], dass eine Ru-Deckschicht keinen Einfluss auf die Kristallisation von CoFeB ausübt, was jedoch von Lu et al. [27, 49] nicht bestätigt werden konnte. Dort wies das CoFe eine (011)-Texturierung auf, was auf eine Kristallisation von der $\mathrm{Ru}$-Deckschicht aus hindeutet.

\subsubsection{Abhängigkeit der Bor-Diffusion vom Deckschicht-Material}

Zum Einfluss der Deckschicht auf die Kristallisation des CoFeB kommt noch der Einfluss auf die Bordiffusion beim Auslagern: 
Eines der am häufigsten verwendeten Deckschicht-Materialien ist Tantal. In HRTEM- und EELS-Untersuchungen von Miyajima et al. [26] zeigte sich an MTJ mit Ta-Deckschicht nach vierstündiger Auslagerung bei $320^{\circ} \mathrm{C}$ eine geringe Borkonzentration im Bereich der Grenzschichten zwischen $\mathrm{MgO}$ und kristallinen CoFe-Regionen. Dagegen ist eine hohe Konzentration im Übergangsbereich von der Elektrode in die Deckschicht und auch leicht in die Ta-Deckschicht hinein zu finden. Hieraus wurde gefolgert, dass Ta die BorDiffusion blockiert. Außerdem konnte am Übergang zwischen Ta und CoFeB eine amorphe 1-2 nm dicke Zwischenschicht aus Ta und Bor gefunden werden. Ein ähnliches Bild findet sich bei Karthik et al. [25] nach einem Auslagerungsschritt bei $500{ }^{\circ} \mathrm{C}$. Hier wurde ebenfalls eine geringe Konzentration von Bor am Übergang zwischen $\mathrm{CoFeB}$ und $\mathrm{MgO}$ gefunden, jedoch wurde in der Barriere selbst kein Bor entdeckt.

Lu et al. [27] untersuchten die Bor-Diffusion mittels Röntgen-Photoelektronenspektroskopie (X-ray Photoelectron Spektroscopy) an Ru-Deckschichtproben, die bei $350{ }^{\circ} \mathrm{C}$ für eine Stunde ausgelagert worden waren. Hier zeigte sich in der gesamten $\mathrm{MgO}$-Schicht und nicht nur am Übergang zu den Elektroden eine hohe homogene Bor-Konzentration. Es wird vermutet, dass das CoFeB vom $\mathrm{Ru}$ aus kristallisiert und eine Bordiffusion in Richtung $\mathrm{MgO}$ begünstigt. Jedoch wird auch erwähnt, dass die MgO-Barriere hier nicht aus stöchiometrischem $\mathrm{MgO}$ besteht und Defekte aufweist, die die Bor-Diffusion begünstigen sollten.

Nutzt man Ti als Deckschicht, führt das Auslagern dazu, dass die zunächst kristalline hcp-Ti-Struktur amorphisiert. In dieser a-Ti-Schicht ist die BorKonzentration sehr hoch. Somit dient das Titan als Senke für die Bor-Diffusion [26]. Darüber hinaus findet sich in der MgO-Barriere Bor in geringer Konzentration. Karthik et al. konnten an Ti-Deckschichten eine Bildung von $\mathrm{Ti}(-\mathrm{B}) \mathrm{O}_{\mathrm{x}}$ mittels EELS nachweisen [28].

Mit NiFe-Deckschichten entsteht laut Miyajima et al. [26] nach dem Auslagern $\left(270{ }^{\circ} \mathrm{C}, 4 \mathrm{Std}\right.$.) eine vollständige fcc-kristalline NiFe-Schicht, die, wie oben bereits erwähnt, einen Einfluss auf die Kristallisation von CoFeB hat. Bor scheint hier vom $\mathrm{CoFeB}$ durch die NiFe-Schicht hindurchzudiffundieren und in der darüberliegenden Ta-Schicht zu segregieren. Wie bei den Ti- und den Ta-Deckschichten wurde auch hier Bor in der Barriere gefunden.

\subsubsection{Einflüsse der unterschiedlichen Herstellungsmethoden von MgO-Barrieren}

In der Praxis werden weitgehend zwei unterschiedliche Herstellungsweisen für MgO-Barrieren benutzt, welche im Anhang B kurz vorgestellt werden: das 
Elektronenstrahlverdampfen und das rf-Sputtern, das in der Literatur und im industriellen Maßstab deutlich öfter zu finden ist [2]. Die beiden Herstellungsarten scheinen jedoch insbesondere an der Grenzfläche zwischen $\mathrm{MgO}$ und $\mathrm{CoFe}$ einen unterschiedlichen Einfluss auf die Segregation von Bor aufzuweisen. Das ist recht problematisch, weil hierdurch der Tunnelprozess durch das $\Delta_{1}$-Band im $\mathrm{MgO}$, das für hohe TMR in diesen Systemen verantwortlich ist, gestört wird [24].

Ebenso erhöht sich der Gesamtwiderstand über eine mit Bor verunreinigte Barriere gegebener Dicke gegenüber einer nicht kontaminierten Barriere [24].

Eine Studie von Cha et al. [31] konnte nachweisen, dass sich bei rf-gesputterten $\mathrm{MgO}$-Barrieren eine signifikante Menge $\mathrm{BO}_{\mathrm{x}}$ in der $\mathrm{MgO}$-Schicht bildet, das nicht beim Elektronenstrahlverdampfen auftrat. Außerdem wiesen die per Elektronenstrahlverdampfen hergestellten MgO-Barrieren eine bessere Kristallinität auf. Auch konnten schärfere Übergänge vom $\mathrm{MgO}$ zum CoFe als bei rf-gesputterten MTJs festgestellt werden, die mehr Korngrenzen im MgO und rauere Übergänge aufweisen.

In späteren Untersuchungen der gleichen Gruppe [32] konnte Boroxid sowohl an der Grenzfläche zwischen $\mathrm{MgO}$ und $\mathrm{CoFe}$, als auch an der Grenzfläche der bei Karthik et al. 25] hergestellten rf-gesputterten Proben gefunden werden. Jedoch wurde hier kein $\mathrm{BO}_{\mathrm{x}}$ in der $\mathrm{MgO}$-Barriere nachgewiesen. Auch bei Schreiber et al. [30] wurde Bor am $\mathrm{MgO} / \mathrm{CoFe}$-Übergang von rf-gesputterten Proben nachgewiesen.

XPS- und EELS-Studien von Kurt et al. [50] an rf-gesputtertem und ebverdampftem $\mathrm{MgO}$ zeigen, dass das Bor immer von der kristallinen Barriere weg diffundiert. Auch in rf-gesputterten MgO-Barrieren wurde kein Bor oder Boroxid zwischen Elektrode und Barriere oder im MgO festgestellt. Zurückgeführt wurde dies auf eine hohe Auslagerungstemperatur von $400{ }^{\circ} \mathrm{C}$ und „hochqualitative“ MgO-Barrieren.

Aufgrund der wenigen Studien und unterschiedlichen Ergebnissen an ebverdampften $\mathrm{MgO}$-Barrieren ergibt sich für den Einfluss der Herstellungsweise der MgO-Barriere noch kein einheitliches Bild. Jedoch gibt es durchaus Diskussionen über die physikalischen Ursachen der Bor-Kontamination, welche durch eine bestimmte Herstellungsart begünstigt werden. Eine mögliche Erklärung für die hohe Konzentration von Bor bzw. Boroxid an der Grenzfläche bzw. im MgO könnte ein Sauerstoffüberschuss sein, der in rf-gesputterten Proben auftritt [30]. Dieser Sauerstoffüberschuss kann bei der Probenherstellung durch ein Ta „presputtern“ reduziert werden. Dabei wird der Einbau von Sauerstoff aus dem Restgas im Vakuum vermindert und das Bor kann an der Grenzfläche um bis zu 50 \% reduziert werden. Eine andere Erklärung ist, dass sich im MgO eine ternäre $\mathrm{Mg}-\mathrm{O}-\mathrm{B}-\mathrm{Phase}$ bildet, die jedoch das Vorhandensein von $\mathrm{Mg}^{+} \mathrm{im}$ 
$\mathrm{MgO}$ erfordert. Hierfür ist aber eine hohe Dichte an Sauerstoffleerstellen nötig [50]. Durch die starke Affinität von Bor und Sauerstoff kann sich darüber hinaus an der Grenzschicht zwischen $\mathrm{CoFe}$ und $\mathrm{MgO}$ oder an Korngrenzen im $\mathrm{MgO}$ ein $\mathrm{BO}_{\mathrm{x}}$ bilden [50].

Somit ist sowohl ein Sauerstoffüberschuss als auch ein -defizit als Ursache denkbar. Man kann jedoch auf jeden Fall davon ausgehen, dass die Stöchiometrie der MgO-Tunnelbarriere einen wichtigen Einfluss aufweist.

\subsubsection{Diffusion weiterer Elemente}

Neben der beim Auslagern bei geringen Temperaturen beabsichtigten Diffusion von Bor in den MTJs treten mit steigender Auslagerungstemperatur unbeabsichtigte, signifikante Diffusionen anderer Elemente auf. So kann bei Temperaturen von $650{ }^{\circ} \mathrm{C}$ eine Diffusion von Fe in die benachbarte Ta-Schicht nachgewiesen werden. Ebenso ist eine Ta-Diffusion bei $600{ }^{\circ} \mathrm{C}$ ins $\mathrm{CoFe}$ und auch in die $\mathrm{MgO}$-Barriere möglich [4]. Ebenso ist eine Oxidation der Ta-Schicht bei Temperaturen von über $500{ }^{\circ} \mathrm{C}$ möglich, wobei auch Sauerstoff im CoFe nachgewiesen werden kann [25]. Besonders die Ta-Diffusion in die Barriere kann zu einem Bruch der $\Delta_{1}$-Symmetrie und letztendlich zur Reduktion des TMR führen. Somit gilt es, diese hohen Temperaturen beim Auslagern generell zu vermeiden. Auch eine Diffusion von $\mathrm{Fe}$ und $\mathrm{Co}$ in die $\mathrm{MgO}$-Barriere ist laut Schreiber et al. [30] mittels Atomsonden-Tomographie (Atom Probe Tomography) nachgewiesen, wobei Eisen aufgrund der höheren Sauerstoffaffinität in höherer Konzentration als Co vorhanden ist. Auch die Diffusion in die Tunnelbarriere hinein hat einen äußerst schädlichen Einfluss auf den TMR. 
$\Gamma_{\text {Kapitel }} 3$

\section{Experimentelle Methoden}

Da es in der Transmissionselektronenmikroskopie eine Vielzahl experimenteller Methoden gibt, werden im nun folgenden Kapitel die in dieser Arbeit benutzten Methoden kurz skizziert und auf deren Nutzung in Kapitel 4 verweisen. Viele Details der angewendeten Methoden können bei Bedarf in der angegebenen Literatur genauer nachgelesen werden.

Abschnitt 3.1 beschreibt die grundlegenden Aspekte der hochauflösenden Transmissionselektronenmikroskopie und deren Limitierungen durch Linsenfehler (s. Abschnitt 3.1.1), die für die in dieser Arbeit durchgeführte Untersuchung von Grenzflächen wichtig sind.

Seit Anfang 2013 ist es an der Universität Göttingen durch die Verfügbarkeit eines aberrationskorrigierten Elektronenmikroskops möglich, Linsenfehler apparativ zu korrigieren. Aus diesem Grund gibt Abschnitt 3.1.2 eine kurze Einführung in die Thematik und einige sich damit eröffnende, neue Möglichkeiten. Eine nicht gerätegebundene Technik, die auftretenden Linsenfehler nachträglich numerisch zu korrigieren, stellt die Objektwellenrekonstruktion dar, welche in Abschnitt 3.1 .3 eingeführt wird.

Die in dieser Arbeit genutzten analytischen Methoden der Energiedispersiven Röntgenspektroskopie (EDX, Abschnitt 3.2.1) sowie der Elektronenenergieverlustspektroskopie (EELS, Abschnitt 3.2.2) werden im Anschluss dargestellt. Hierbei liegt der Fokus auf der ebenfalls erst seit Anfang 2013 in Göttingen verfügbaren EELS-Methode und deren Vorteile in dem hier verwendeten Materialsystem.

Eine für diese Arbeit zentrale Rolle spielt das von Borgardt, Plikat, Thiel, Schröter und Seibt [13, 14, 21, 51, 22, 52, 53] entwickelte und genutzte APPVerfahren. Es ermöglicht eine Bestimmung der langreichweitigen Ordnung an der Grenzfläche. Diese Methode wurde im Rahmen dieser Arbeit weiterent- 
wickelt und an das hier untersuchte Materialsystem aus kristallinem $\mathrm{MgO}$ und amorphem CoFeB angepasst. Abschnitt 3.3 stellt das Verfahren ausführlich vor.

Die Vorgehensweise, mit der die für alle TEM-Untersuchungen notwendigen dünnen, homogenen TEM-Lamellen hergestellt werden, wird in Abschnitt 3.4 diskutiert.

\subsection{Hochauflösende Transmissionselektronenmikroskopie}

Bei der Hochauflösenden Transmissionselektronenmikroskopie (HRTEM) handelt es sich um eine Methode, die es erlaubt, atomare Strukturen zu untersuchen und zu charakterisieren [54, 55, 56]. Dabei gibt es eine Vielzahl unterschiedlicher Techniken, die sich je nach Einsatzzweck zum Teil auch miteinander kombinieren lassen. So bietet zum Beispiel die abbildende Transmissionselektronenmikroskopie die Möglichkeit, Ortsauflösungen besser als $1 \AA$ $\mathrm{zu}$ erreichen und damit u.a. Kristalldefekte zu untersuchen. Hierbei wird eine einfallende Elektronenwelle $\psi_{0}$ beim Durchgang durch die Probe durch das Streupotential $V$ zur austretenden Objektwelle $\psi$ modifiziert. Beim weiteren Durchgang durch das Mikroskop wird die austretende Objektwelle durch die auftretenden Linsenfehler zur Bildwelle $\psi_{B}$ verändert. Bei der Aufzeichnung der komplexen Bildwelle $\psi_{B}$ auf einer CCD-Kamera wird die Intensität in der Bildebene $I=\left|\psi_{B}\right|^{2}$ aufgezeichnet, wodurch die Phaseninformation in der komplexen Bildwelle verlorengeht.

In der Regel sind die daraus erhaltenen Abbildungen weder direkt interpretierbar noch kann aus einzelnen Abbildungen eine atomare Struktur direkt quantitativ charakterisiert werden.

Der Grund für die schlechte direkte Interpretierbarkeit von HRTEM-Abbildungen liegt im Wesentlichen in der Limitierung durch Linsenfehler. Ebenso sorgt eine Mehrfachstreuung in der Probe für einen nichtlinearen Zusammenhang zwischen dem Probenpotential und der Bildwelle. Dieser Effekt lässt sich nur durch möglichst dünne Proben reduzieren. Auch der Verlust der Phaseninformation bei der Bildaufzeichnung sorgt für eine schlechte Interpretierbarkeit von elektronenmikroskopischen Abbildungen.

In der Objektwelle sind im Gegensatz zur Bildwelle diese Informationen enthalten. Um dieses inverse Problem zu lösen und die Objektwelle zu bekommen bieten sich mehrere holographische Methoden [57] an:

- Bei der „Off-Axis“-Holographie wird die Bildwelle mit einer Referenzwelle überlagert und daraus ein Hologramm erzeugt. Durch eine numerische 
Korrektur der Auswirkungen der Linsenfehler ist es anschließend möglich, hieraus die komplexe Objektwelle zu bestimmen. Da bei diesem Verfahren auch die tieffrequenten Bildanteile mit übertragen werden, ist es ebenfalls möglich, elektrische und magnetische Felder innerhalb der Probe zu bestimmen. Dieses Verfahren ist an den in dieser Arbeit verwendeten Elektronenmikroskopen nicht möglich; deswegen sei für weitere Informationen auf die Literatur verwiesen [58, 57].

- Ein anderer Weg die ursprüngliche Objektwelle zu erhalten, ist die Objektwellenrekonstruktion. Dabei wird aus einer Serie von mehreren Abbildungen, die unter verschiedenen Abbildungsbedingungen aufgenommen wurden, die Objektwelle mittels statistischer Verfahren numerisch bestimmt. Hier kann man die Objektwelle um den Effekt der Linsenfehler numerisch korrigieren. Eine nähere Beschreibung zu diesem Verfahren ist unter Abschnitt 3.1.3 zu finden.

- Eine weitere Möglichkeit bietet der iterative Vergleich von Abbildungsserien. Dabei geht man einen Schritt weiter und rekonstruiert nicht die Objektwelle, sondern direkt die atomare Struktur, die diese Abbildungen hervorgerufen haben könnte. Diese Methode wird in Abschnitt 3.3 zur Bestimmung der langreichweitigen Ordnung an der Grenzfläche angewendet. Dabei werden ganze Serien von experimentellen Abbildungen mit simulierten Abbildungen verglichen. Die simulierten Abbildungen basieren auf einem parametrisierten Strukturmodell, dessen Parameter in einem iterativen Prozess angepasst werden.

\subsubsection{Aberrationen}

Einer der Hauptgründe, weshalb HRTEM-Abbildungen nicht intuitiv interpretierbar sind, liegt also in der Veränderung der Objektwelle durch Linsenfehler, hauptsächlich die der Objektivlinse. Diese Linsenfehler äußern sich in einer Verschmierung der Objektwelle und können durch eine Phasenverschiebung mathematisch beschrieben werden. Eine ausführliche Darstellung möglicher Aberrationen findet sich u.a. bei Erni [59].

Linsenfehler kann man in kohärente und inkohärente Linsenfehler einteilen:

- Für Kohärente Abbildungsfehler, die durch die eingesetzten Elektronenlinsen entstehen, gibt es größtenteils Korrektorsysteme. Zu den wichtigsten kohärenten Abbildungsfehlern gehören: 
- Der axiale Astigmatismus entsteht durch ein nicht-rotationssymmetrisches Linsenfeld. Eine solche Linse weist in verschiedene Richtungen unterschiedliche Brennweiten auf, weshalb von einem Objektpunkt aus kommende Strahlen in der Bildebene nicht in einem Punkt vereint werden. Den zweizähligen und dreizähligen Astigmatismus kann man in allen gebräuchlichen Mikroskopen mittels spezieller Stigmatorlinsen korrigieren.

- Befindet sich der Objektpunkt nicht auf der optischen Achse, sondern mit einigem Abstand dazu, kommt es zu einer asymmetrischen Verschmierung des Strahlbündels in der Brennebene durch das außeraxiale Koma. Bis zu einem bestimmten Einfallswinkel lässt sich das Koma durch ein „Coma-Free-Alignment" kompensieren.

- Durch die sphärischen Aberration $C_{s}$ (auch Öffnungsfehler genannt) werden beim Durchgang durch die Linse die achsenferneren Strahlen nicht auf den gleichen Punkt fokussiert, die näher an der optischen Achse liegende. Die sphärische Aberration ist in der Regel eine konstante Größe für ein Mikroskop, jedoch sind seit einiger Zeit kommerzielle Korrektorsysteme verfügbar, die aus mehreren Multipol- und Transferlinsen bestehen. Ein solches System samt komplexer Steuerung ist in dem hier verwendeten FEI Titan 80300 ETEM G2 installiert. Es bietet nicht nur die Möglichkeit, den Öffnungsfehler zu minimieren, sondern ebenfalls auch alle weiteren Linsenfehler bis zur 3. Ordnung zu bestimmen und zu korrigieren.

- Bei den inkohärenten Abbildungsfehlern wird die Phasenbeziehung zwischen den Elektronen durch Änderung der Phasendifferenz über die Zeit gestört. Diese inkohärenten Abbildungsfehler können eingeteilt werden in Aberrationen, die aufgrund der räumlichen Kohärenz des Strahls (durch die endliche Größe der Elektronenquelle) entstehen und solche, die durch zeitliche Kohärenz (durch Schwankungen der Hochspannung, der Linsenströme, etc.) auftreten:

- Bei der chromatischen Aberration $C_{c}$ handelt es sich um den Abbildungsfehler, der dadurch entsteht, dass Strahlelektronen mit geringfügig unterschiedlichen Energien auf unterschiedliche Punkte fokussiert werden. Auch hierfür gibt es bereits ein experimentelles Korrektorsystem, welches aber aktuell weltweit erst in wenigen Mikroskopen installiert ist!1 Eine Möglichkeit die chromatischen

${ }^{1}$ Das erste Mikroskop mit installiertem $C_{c}$-Korrektor, befindet sich im Argonne National Lab, zwei weitere Geräte, das TEAM0.5 und das TEAM1-Gerät, stehen im Lawrence 
Fehler zu verringern, anstatt sie zu korrigieren, besteht darin, die Energiebreite des Elektronenstrahls zu verringern. Dazu kann man einen Monochromator (sofern vorhanden) nutzen, der üblicherweise dafür genutzt wird, die ursprüngliche Energiebreite des Strahls von $\leq 0,8 \mathrm{eV}$ auf $\leq 0,2 \mathrm{eV}$ zu verringern, um eine bessere Energieauflösung beim EELS (s. Abschnitt 3.2.2) zu erzielen. Da bei der Nutzung des Monochromators nur noch wenige Elektronen zur Abbildung der Probe genutzt werden, verliert der Elektronenstrahl an Helligkeit, was eine höhere Belichtungszeit zur Folge hat. Aus diesem Grund wird der Monochromator im Rahmen dieser Arbeit nicht verwendet.

Einen starken Einfluss auf die direkte Interpretierbarkeit von elektronenmikroskopischen Abbildungen hat die sogenannte Delokalisierung.

Delokalisierung bedeutet, dass Punkte in der Probe in einem ausgedehnten Bereich in der Abbildung transformiert werden.

Die Ausdehnung der Delokalisierung kann unter Umständen mehrere Nanometer betragen, wie ein anschauliches Beispiel in [21] zeigt. Die Delokalisierung $\Delta R$ lässt sich für ein nicht-aberrationskorrigiertes Mikroskop beschreiben mit:

$$
\Delta R=\lambda q\left(\Delta f+C_{s} \lambda^{2} q^{2}\right)
$$

wobei $\Delta f$ der Defokus 2 , $C_{s}$ die sphärische Aberration, $\lambda$ die Wellenlänge der Elektronen und $q$ ein Punkt im reziproken Raum ist [59]. Man sieht, dass der Effekt der Delokalisierung größer wird, wenn man sich vom optimalen Defokus entfernt. Außerdem kann man den Effekt durch eine höhere Beschleunigungsspannung verringern. Diese lässt sich aber nur apparativ ändern und erhöht zudem die Gefahr der Strahlschädigung der Probe. Allerdings lässt sich die Delokalisierung auch durch eine Reduktion der sphärischen Aberration erreichen. Da diese im Allgemeinen eine konstante, geräteinhärente Größe ist, lässt sie sich nur in abbildungskorrigierten TEMs verändern. Aufgrund der direkteren Interpretierbarkeit von abbildungskorrigierten Abbildungen durch die verringerte Delokalisation und der höheren Auflösung durch Korrektur der Linsenfehler bis zur 3. Ordnung wurden die in dieser Arbeit durchgeführten Untersuchungen der Grenzfläche zwischen $\mathrm{MgO}$ und $\mathrm{CoFe}(\mathrm{B})$ an einem aberrationskorrigierten Mikroskop durchgeführt.

Berkeley National Lab. Desweiteren steht ein $C_{c}$-korrigiertes Mikroskop am Ernst RuskaCenter in Jülich.

${ }^{2} \mathrm{Im}$ Falle eines Überfokus ist $\Delta f>0$ und im Fall des Unterfokus ist $\Delta f<0$. 
Mathematisch lässt sich die Übertragung der Objektwelle $\psi_{O}$ im Mikroskop in die Bildwelle $\psi_{I}$ durch die beugungswinkelabhängige Phasenmodulationsfunktion $T(\vec{q})$ beschreiben, in die die Linsenfehler des Mikroskops eingehen:

$$
\tilde{\psi}_{I}(\vec{q})=\tilde{\psi}_{O}(\vec{q}) \cdot T(\vec{q})
$$

wobei die komplexe Phasenmodulationsfunktion $T(\vec{q})$ gegeben ist durch:

$$
T(\vec{q})=\exp \{i \chi(\vec{q})\}
$$

wobei für nicht-aberrationskorrigierte Mikroskope gilt:

$$
\chi(\vec{q})=\pi \Delta f q^{2} \lambda+\frac{1}{2} \pi C_{s} q^{4} \lambda^{3}
$$

Hier dominieren die sphärischen Aberration und der Defokus. Für korrigierte Mikroskope ist $\chi(\vec{q})$ deutlich komplexer und kann bei Erni [59] nachgelesen werden. Dort finden sich ebenfalls Erläuterungen über Linsenfehler höherer Ordnung, die hier nicht angesprochen werden.

Der erste Nulldurchgang des Imaginärteils der Phasenmodulationsfunktion beim „Scherzer-Fokus ${ }^{6}{ }^{3}$ definiert die Punktauflösung eines Mikroskops. Dies ist der Punkt bis zu welchem die Raumfrequenzen phasengleich, d.h. ohne Kontrastumkehr, abgebildet werden. Zusätzlich zu den kohärenten wirken sich auch die inkohärenten Aberrationen auf die Kontrastübertragung aus. Die zeitlich inkohärenten Aberrationen $E_{T}(\vec{q})$ und räumlich inkohärenten Aberrationen $E_{S}(\vec{q})$ sorgen im einfachsten Fall jeweils für eine Dämpfungseinhüllende, so dass sich für die Kontrasttransferfunktion $C T F(\vec{q})$ ergibt:

$$
C T F(\vec{q})=T(\vec{q}) \cdot E_{S}(\vec{q}) \cdot E_{T}(\vec{q})
$$

Das Informationslimit ist durch diese inkohärenten Aberrationen limitiert und definiert als der Wert, bei dem die Dämpfungseinhüllende auf $e^{-2} \approx 13 \%$ abgefallen ist [21].

\subsubsection{Aberrationskorrigierte Elektronenmikroskopie und ihre Anwendung}

Die Entwicklung eines Korrektorsystems für kohärente Linsenfehler war ein Meilenstein auf dem Weg zur modernen Transmissionselektronenmikroskopie.

\footnotetext{
${ }^{3}$ Für den Scherzer-Fokus gibt es verschiedene Definitionen, hier wird die Definition $\Delta f_{s}=$ $-\sqrt{\frac{3}{2} \lambda C_{s}}$ genutzt.
} 
In der konventionellen Hochauflösenden Elektronenmikroskopie beschränkt die sphärische Aberration die erzielbare Auflösung. Bereits 1936 zeigte Otto Scherzer in seinem berühmten Theorem [60], dass eine positive sphärische Aberration nur minimierbar, nicht aber vermeidbar ist, wenn die folgenden Bedingungen erfüllt sind:

- Die Linsenfelder sind rotationssymmetrisch.

- Das elektromagnetische Feld weist keine Raumladung auf.

- Die elektromagnetischen Felder sind statisch.

Diese Bedingungen treten typischerweise für alle runden Elektronenlinsen auf. Sobald jedoch eine dieser Bedingungen nicht erfüllt ist, besteht die Möglichkeit, die mit der sphärischen Aberration einhergehenden Limitierungen zu vermeiden. Der letztendlich erfolgreichste Ansatz war, nichtrotationssymmetrische Linsen einzusetzen. Born und Wolf [61] beschrieben in ihrem Additionstheorem, dass Linsenfehler additiv sind. Durch diese Eigenschaft ist es nicht notwendig, aberrationsfreie Linsen zu entwickeln; vielmehr genügt es, diese Linsenfehler in folgenden Linsen, z.B. durch ein Hexapollinsen-Korrektorsystem, basierend auf einer Idee von Scherzer und Rose [60, 62, zu korrigieren. Durch diese Hexapollinsen wird eine negative sphärische Aberration hinzugefügt, welche die vorhandene, positive kompensiert. Kommerzielle Korrektoren, wie der in dem hier verwendeten FEI TITAN der Firma CEOS, setzen diese Idee zusammen mit weiteren Transferlinsen um [63]. Das Prinzip ist in Abbildung 3.1 dargestellt. Dieser Korrektor dient der Korrektur in HRTEM-Abbildungen und sitzt in diesem Fall hinter der Objektivlinse. Für die Korrektur von STEM-Abbildungen ist es dagegen notwendig, die sphärische Aberration der Beleuchtung vor der Objektivlinse zu korrigieren.

Mit einem Korrektorsystem ist es nun sowohl möglich, die Punktauflösung dem Informationslimit des Mikroskops anzugleichen, als auch die mit der sphärischen Aberration zusammenhängende Delokalisierung zu minimieren. Weiterhin ist es möglich, den Abstand zwischen dem oberen und dem unteren Objektivlinsenpolschuh zu vergrößern. So steht vor allem für tomographische Anwendungen, in denen ein hoher Kippwinkel der Probe erforderlich ist und bei in-situ Experimenten mehr Platz zwischen den Objektivlinsen-Polschuhen zur Verfügung. Ohne Aberrations-Korrektor hätte ein größerer Polschuhabstand einen höheren $C_{s}$-Koeffizienten zur Folge, der nun jedoch wieder korrigiert werden kann. 


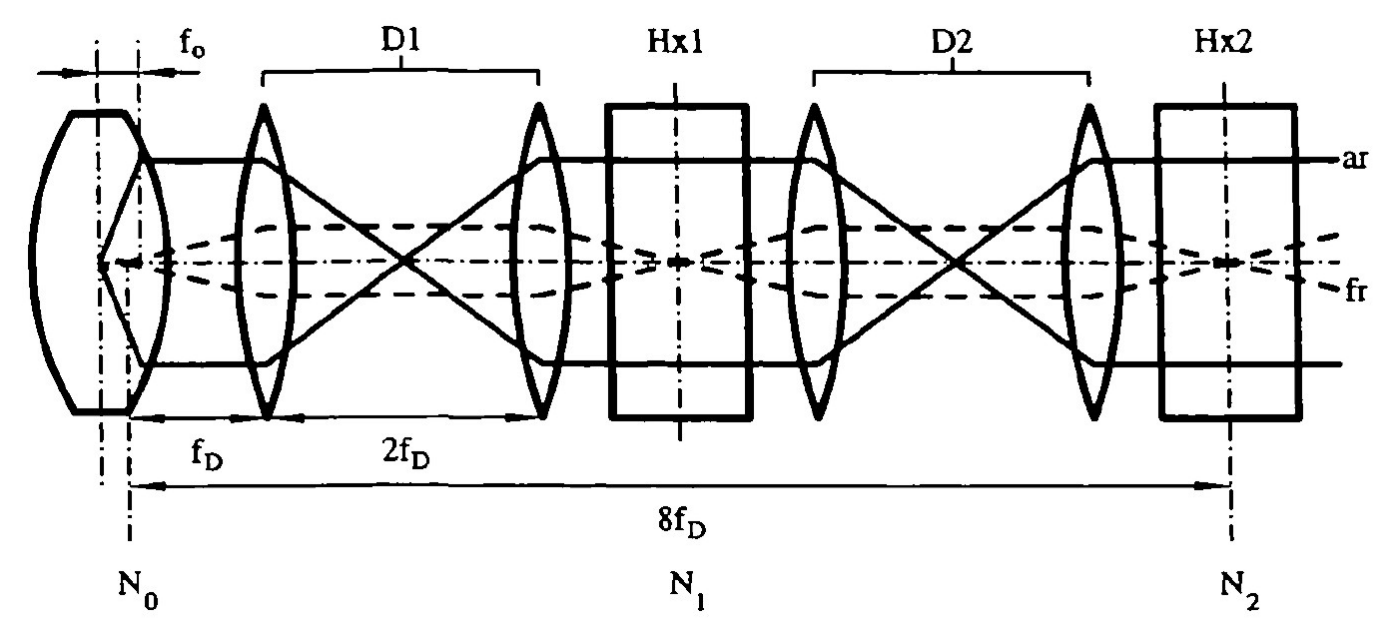

Abbildung 3.1.: $C_{s}$-Korrektorsystem bestehend aus der unteren Objektivlinse links und den Transferlinsen D1 und D2 sowie den beiden Hexapolen Hx1 und Hx2. Aus [63].

Durch die Korrektur ist die sphärische Aberration keine Konstante, sondern eine Variable und muss zusammen mit weiteren, störenden Linsenfehlern gemessen und korrigiert werden. Dieser Umstand macht die Ansteuerung des Korrektors sehr komplex und wird deshalb in der Regel von der Software übernommen. Die Korrektur der Linsenfehler erfolgt im Allgemeinen nie vollständig, weshalb nach der Messung der Linsenfehler abgeschätzt wird, welche Linsenfehler überwiegen und zu korrigieren sind, bis ein tolerierbarer Restfehler übrig bleibt. Die Linsenfehler werden auf einer dünnen, amorphen Probenstelle mittels eines Zemlin-Tableaus (s. Abb. 3.2) bestimmt. ${ }^{4}$ Hierbei wird der Elektronenstrahl bei einigen $100 \mathrm{~nm}$ Unterfokus relativ zur optischen Achse verkippt und auf der CCD-Kamera abgebildet. In der Fouriertransformierten dieser Abbildung sind mehrere Thon-Ringe der amorphen Probe sichtbar. Da die Aberrationen sich mit der Strahlverkippung ändern, können diese bei einer hinreichend großen Anzahl an Abbildungen und einer bekannten Strahlverkippung bestimmt werden [64, 65]. Nach der Messung der Linsenfehler werden diese sukzessive minimiert. Danach schließen sich in einem iterativen Prozess

\footnotetext{
${ }^{4}$ Der Korrekturprozess dauert relativ lange (ca. 45-60 Minuten). In dieser Zeit kann sich der amorphe Bereich der Probe unter Beleuchtung verändern. Da in per FIB hergestellten TEM-Proben häufig kein geeignet großer, dünner amorpher Bereich zur Verfügung steht (oder gar wie hier selbst im Fokus des Interesses steht), bietet es sich an, zusätzlich zum FIB-Probenträger ein halbkreisförmiges Stück Kohlenstoff-Folie einzubauen. An dieser kann der Korrektor sorgfältig eingestellt werden, ohne die eigentliche Probe durch Strahlschädigung zu gefährden.
} 
weitere Aberrationsmessungen mittels Zemlin-Tableau und Korrekturen an, bis eine ausreichend korrigierte Übertragung der Objektwelle gegeben ist. Dabei können alle Linsenfehler bis zur 3. Ordnung $5^{5}$ korrigiert werden.


Abbildung 3.2.: Links: Diffraktogramm einer amorphen Kohlenstofffolie bei $\Delta f=-180$ nm. Rechts: Zemlin-Tableau zur Bestimmung der Linsenfehler bestehend aus 17 Diffraktogrammen. Die äußeren Diffraktogramme sind bei einer Strahlverkippung von 24 mrad aufgenommen worden.

\subsubsection{Abbildung mit negativer sphärischer Aberration}

Nun ist es mit einem aberrationskorrigierten TEM nicht nur möglich, die sphärische Aberration $\mathrm{zu}$ bestimmen und $\mathrm{zu}$ minimieren, sondern, da diese nun variabel ist, ist es ebenfalls möglich, einen negativen Wert des Öffnungsfehlers einzustellen.

Mit Hilfe dieser abbildenden, negativ sphärischen Aberrationstechnik (negativ spherical aberration imaging, kurz: NCSI) konnte in Proben aus $\mathrm{SrTiO}_{3}$ und $\mathrm{YBa}_{2} \mathrm{Cu}_{3} \mathrm{O}_{7}$ bei Überfokus gezeigt werden, dass dies zu einer besseren Sichtbarkeit von Sauerstoffatomen in der Nähe von stark streuenden Metallatomen führt [66]. Sogar eine Quantifizierung der Sauerstoffkonzentration an Zwillingskorngrenzen in $\mathrm{BaTiO}_{3}$-Systemen konnte mit dieser Methode ermöglicht werden [67]. Der beobachtete hohe Kontrast in diesen Abbildungen entsteht laut

${ }^{5}$ 1. Ordnung: zweizähliger Astigmatismus; 2. Ordnung: dreizähliger Astigmatismus, Axiale Koma ; 3. Ordnung: vierzähliger Astigmatismus, sternförmige Aberration, sphärische Aberration. 


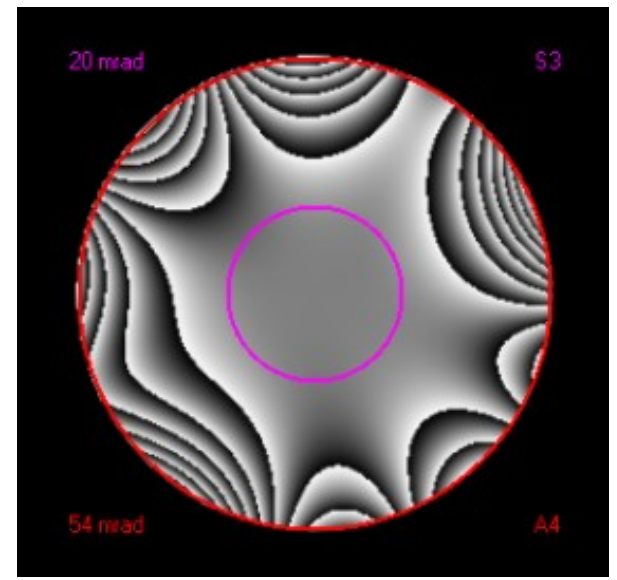

Abbildung 3.3.: Visualisierung der in Abb. 3.2 bestimmten Aberrationen in einer Phasenplatte. $A_{1}=1,9 \mathrm{~nm}$ (zweizähliger Astigmatismus), $A_{2}=31,7 \mathrm{~nm}$ (dreizähliger Astigmatismus), $B_{2}=1,1 \mathrm{~nm}$ (Axiale Koma), $C_{3}=1,7 \mu \mathrm{m}$ ( $C_{s}$ Sphärische Aberration), $A_{3}=804 \mathrm{~nm}$ (vierzähliger Astigmatismus), $S_{3}=606 \mathrm{~nm}$ (sternförmige Aberration), $A_{4}=66,5 \mu m$ (5-zähliger Astigmatismus).

Urban et al. [68] aus einer vorteilhaften Kombination aus Amplitudenkontrast (resultierend aus „Channeling“-Effekten) und Phasenkontrast.

Ausführlichere Darstellungen über Aufbau und Funktionsweise von $C_{s}$-Korrektoren finden sich in der Literatur [59, 69]. Details zur Abbildung leichter Elemente mittels NCSI-Technik finden sich unter anderem bei Jia et al. [66, 67, Urban et al. [68] oder Kisielowski et al. [70].

\subsubsection{Objektwellenrekonstruktion}

Wie zu Beginn des Abschnitts 3.1 skizziert, bietet auch die Objektwellenrekonstruktion die Möglichkeit, die Objektwelle numerisch aus einer Serie von Aufnahmen mit unterschiedlicher Defokussierung zu bestimmen. Da bei dieser Rekonstruktion die auftretenden Linsenfehler nachträglich und sehr genau korrigiert und dazu die Phaseninformation in der komplexen Objektwelle wiedergewonnen werden können, hat diese komplexe Methode auch nach Einführung von $C_{s}$-Korrektoren ihre Vorteile nicht verloren. Tillmann et al. [71] zeigten, dass die Kombination beider Techniken gerade bei der Untersuchung der Struktur von Defekten in Kristallgittern oder bei Heterogrenzflächen große Vorteile aufweist. In dieser Arbeit wird die Methode bei der Untersuchung der kristallin/kristallinen Grenzfläche von $\mathrm{MgO}$ und CoFe in Abschnitt 4.1.1 angewendet, um die Gitterabstände an der Grenzfläche genau bestimmen zu können.

Zur Rekonstruktion der Objektwelle wird in dieser Arbeit auf das Programm 
TrueImage Professional 2.0.1 (Build 7) von FEI zurückgegriffen [72, 73, 74, 75]: Es führt zuerst mittels Kreuzkorrelation eine Driftkorrektur der Abbildungen einer Defokusserie durch. Anschließend versucht das Programm, durch Verwendung zweier verschiedener Methoden, die Objektwelle zu berechnen. Dazu kehrt es mittels Paraboloid-Methode (PAM) den Abbildungsprozess um und filtert nur den linearen Anteil der Abbildungen heraus. Der nicht-lineare Anteil, der als Störung betrachtet wird, wird unterdrückt. Näheres zur PAM-Methode findet sich bei Op de Beeck et al. [76] und Saxton et al. [77.

Im Anschluss hieran wird die Maximum-likelihood-Methode (MAL) angewendet. Diese versucht in einem iterativen Prozess die für die gegebenen experimentellen Abbildungen wahrscheinlichste Objektwellenfunktion zu finden. Als Startwert für diese Methode dient die Rekonstruktion des PAM-Algorithmus. Mit diesem Verfahren ist es möglich, nicht nur die linearen, sondern auch die nicht-linearen Abbildungsanteile zu rekonstruieren. Somit eignet sich diese Methode auch gut für Proben mit dynamischer Vielfachstreuung, also dickere Proben oder in niedrigen Zonenachsen orientierte kristalline Materialien. Diese Methode wurde ursprünglich von Kirkland et al. [78] publiziert und von Coene [72] weiterentwickelt. Näheres dazu findet sich auch bei Lentzen et al. [79].

Im Gegensatz zu der in Abschnitt 3.3 beschriebenen Methode, in der die atomare Struktur der Probe direkt bestimmt wird, können mit Hilfe der Objektwellenrekonstruktion nicht immer direkte Rückschlüsse auf die zu Grunde liegende Struktur der Probe gezogen werden, da hier hier nur die Austrittswelle bestimmt wird. Mehrere Untersuchungen zeigten [74, 80, 81, 82], dass direkte Rückschlüsse auf die Struktur der Probe nur für Phasenobjekte, also Proben, in denen die Wechselwirkungen zwischen Elektronenwelle und Probenpotential nur eine Phasenänderung bewirken, möglich sind. Um Rückschlüsse auf die zugrunde liegende Struktur zu erhalten, muss im Anschluss an die Objektwellenrekonstruktion ein Vergleich mit simulierten Objektwellen durchgeführt werden. 


\subsection{Analytische Transmissionselektronenmikroskopie}

In der Analytischen Transmissionselektronenmikroskopie werden inelastische Streuprozesse ausgenutzt, um Informationen über die chemische Zusammensetzung der Probe zu erhalten. Die in den folgenden beiden Abschnitten diskutierten Methoden der Energiedispersiven Röntgenspektroskopie (EDX, s. Abschnitt 3.2.1) und der Elektronen-Energieverlustspektroskopie (EELS, s. Abschnitt 3.2.2) werden häufig unter Verwendung des STEM-Modus (Scanning Transmission Electron Microscopy) des Mikroskops betrieben. In dieser Betriebsart wird der Elektronenstrahl auf der Probe fokussiert und über die Probe gerastert. Somit können die durch die analytischen Methoden gewonnenen Informationen einer bestimmten Strahlposition zugeordnet werden.

\subsubsection{Energiedispersive Röntgenspektroskopie}

Im Folgenden wird eine kurze Einführung in die Energiedispersive Röntgenspektroskopie gegeben: Eine detailliertere Darstellung findet sich unter [54].

Durch die Bestrahlung der Probe mit einem mehrere kV schnellen Elektronenstrahl werden in den Probenatomen Elektronen von den inneren Schalen auf die äußere Schale angehoben. Bei der folgenden Relaxation wird u.a. charakteristische Röntgenstrahlung ausgesendet, die mit Hilfe eines Detektors in Probennähe gemessen werden kann. Dabei setzt sich das gemessene Signal aus den Anteilen der kontinuierlichen Bremsstrahlung und der charakteristischen Röntgenstrahlung zusammen. Der Bremstrahlungshintergrund wird durch das Abbremsen der Strahlelektronen in der Probe hervorgerufen und ist bis zur maximalen Beschleunigungsspannung der Strahlelektronen im Spektrum vorhanden, dabei überlagert es die charakteristische Röntgenstrahlung.

Zur quantitativen Auswertung wird in der Regel die Methode von Cliff und Lorimer [83] benutzt, bei der die Intensitätsverhältnisse verschiedener charakteristischer Linien nach Abzug des Hintergrundes ausgewertet werden. Die Zusammensetzung lässt sich durch die Cliff-Lorimer-Gleichung

$$
\frac{C_{A}}{C_{B}}=k_{A B} \frac{I_{A}}{I_{B}}
$$

bestimmen. Hier sind $I_{x}$ die Intensitäten der auszuwertenden Elemente und $C_{x}$ deren Gewichtsprozente. $k$ wird auch der Cliff-Lorimer-Faktor genannt, der einen Sensibilitätsfaktor und keine Konstante darstellt. Bei dieser Methode wird angenommen, dass es sich um eine dünne Probe handelt, in der Absorptions- und Fluoreszenzeffekte keinen wesentlichen Einfluss haben. 
Zur qualitativen Auswertung können im STEM-Modus auch Linienprofile oder zweidimensionale Elementverteilungskarten erstellt werden. Diese Methode wird in Abschnitt 4.3 benutzt, um die Elektromigration beim dielektrischen Zusammenbruch von MTJs zu untersuchen. Dazu werden im Röntgenspektrum Energiefenster um eine zu messende, charakteristische Linie erstellt und der Elektronenstrahl entweder entlang einer Strecke oder eines zweidimensionalen Bereichs geführt. In neueren Systemen wie dem OXFORD X-MAX80, welches im FEI Titan ETEM installiert ist, ist es nicht mehr nötig, vorher Energiefenster zu definieren. Hier wird von jedem Punkt der Probe das gesamte Röntgenspektrum aufgezeichnet. Erst zur Auswertung können Energiefenster zur qualitativen Darstellung ausgewählt werden. Der Vorteil dieses Systems ist, dass jederzeit auch eine quantitative Auswertung möglich ist, da von jedem Punkt das volle Spektrum vorhanden ist.

\subsubsection{Elektronenenergieverlustspektroskopie}

Als weitere analytische Methode steht im FEI Titan ETEM die Elektronenenergieverlustspektroskopie (EELS) zur Verfügung. Eine umfassende Darstellung dieser Methode findet sich in der Literatur u.a. in Egerton [84].

EELS wird oft als komplementäre Methode zu EDX bezeichnet. Hier werden keine Sekundäreffekte wie die charakteristische Röntgenstrahlung bei EDX aufgezeichnet, sondern die Energieverteilung der transmittierten Elektronen analysiert. Diese inelastischen Prozesse entstehen, wie bei EDX, durch CoulombWechselwirkungen der gestreuten Strahlelektronen mit den Probenelektronen der inneren und äußeren Schalen. Die für Festkörperelektronen erlaubten energetischen Zustände und Übergänge in den Probenatomen resultieren in charakteristischen Ionisationskanten in den EELS-Spektren.

Um EELS-Spektren zu erhalten, ist ein Energiefilter nötig. Im FEI Titan ETEM ist ein Gatan Quantum ER965 installiert, der sich unterhalb der Elektronensäule befindet. Hinter einer wählbaren Eingangsapertur wird der Elektronenstrahl in einem magnetischen Prisma aufgeweitet und nach Durchgang durch mehrere Multipolprojektionslinsen auf einer CCD-Kamera abgebildet. Hinter dem magnetischen Prisma besteht ebenfalls die Möglichkeit, eine verstellbare Schlitzblende einzuführen, um einen bestimmten Energiebereich abzubilden (s. Abb. 3.4).

Die Energieauflösung des EELS-Signals wird durch zwei Faktoren beeinflusst: die eingestellte Dispersion und die Energiebreite des Elektronenstrahls.

Die Energiebreite des Elektronenstrahls ohne Wechselwirkung mit der Probe wird im Wesentlichen durch die verwendete Elektronenquelle vorgegeben. Bei der in diesem Gerät verwendeten XFEG-Elektronenquelle liegt die erzielba- 


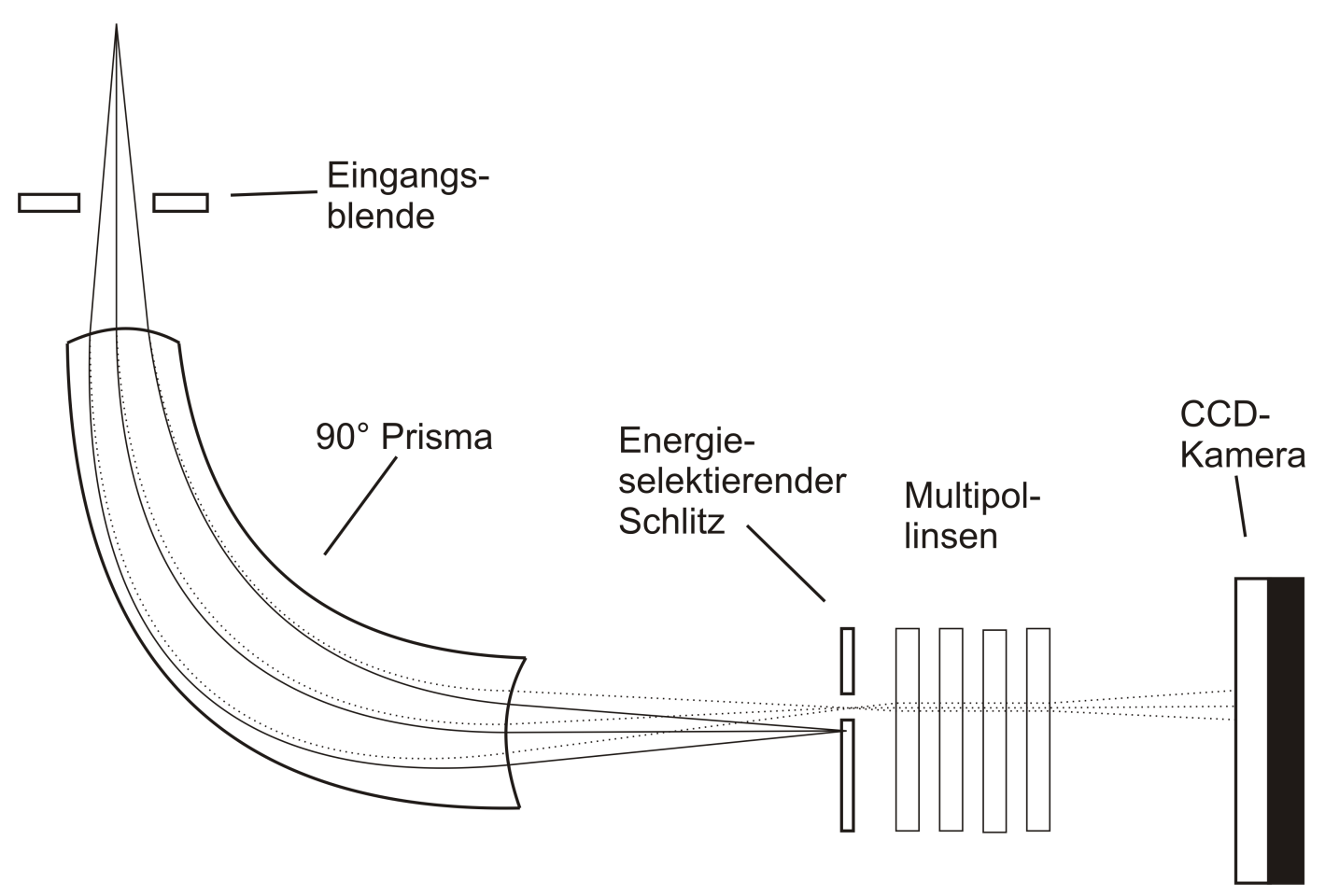

Abbildung 3.4.: Schematische Darstellung eines TEM-Energiefilters.

re Energiebreite des Elektronenstrahls bei etwa $0,7-0,8 \mathrm{eV}$ bei $300 \mathrm{kV}$ Beschleunigungsspannung. Höhere Energieauflösungen sind nur mit eingeschaltetem Monochromator möglich. Dann liegt die erzielbare Energiebreite bei $<0,2 \mathrm{eV}$ bei $300 \mathrm{kV}$ Beschleunigungsspannung. Bei der qualitativen Auswertung des EELS-Signals ist die Energieauflösung nicht von primärem Interesse. Erst bei der Betrachtung des „Energy Loss Near Edge Spectrums“ (ELNES, Absorptionskantenfeinstruktur) ist die Energieauflösung des Systems von größerer Bedeutung.

Die Energiebreite des Elektronenstrahls gibt somit die kleinste zu erzielende Energieauflösung vor. Diese ist jedoch nur bei den kleinsten Dispersionen des Detektors nutzbar. Die Dispersion gibt die Aufweitung des Energiespektrums auf die zur Verfügung stehenden 2048 Kanäle des Detektors an. Bei kleinen Dispersionen kann nur ein sehr kleiner Energiebereich mit großer Energieauflösung dargestellt werden. Je größer die Dispersion und der damit dargestellte Energiebereich ist, umso geringer ist die zur Verfügung stehende Energieauflösung. Folglich ist die hohe Energieauflösung des Monochromators nur im Zusammenspiel mit kleinen Dispersionen sinnvoll. Möchte man jedoch charakteristische Ionisationskanten mehrerer Elemente simultan messen, um z.B. 
das stöchiometrische Verhältnis zueinander zu bestimmen, muss eine größere Dispersion genutzt werden.

Schon bei geringeren Energieauflösungen von etwa 1,5 eV enthält das EELSSpektrum bereits weitere nützliche Informationen. So enthält der Bereich innerhalb von etwa $50 \mathrm{eV}$ hinter der Absorptionskante die Feinstruktur. Diese Feinstruktur ist beeinflusst von den Valenzzuständen des ionisierten Atoms und den umgebenen Atomen. So können bei ELNES-Untersuchungen von Bor Aussagen zum Oxidationszustand gemacht werden [85].

Ähnlich wie beim EDX ist der Hauptbetriebsmodus des TEM bei EELSMessungen der STEM-Betrieb. Damit ist eine Zuordnung des Probenortes und des gemessenen Spektrums gegeben, ebenfalls sind Linienprofile und Elementverteilungskarten möglich. Jedoch kann man den Energiefilter auch unter Abbildungsbedingungen betreiben ohne die Ortsinformationen zu verlieren. In diesem EFTEM-Modus (Energy-Filtered TEM) wird hinter der energiedispersiven Ebene mit der nachfolgenden Optik eine Abbildung erzeugt, zu der nur Elektronen mit einem bestimmten Energieverlust beigetragen haben. Nimmt man nun sehr viele Abbildungen mit unterschiedlichen Energieverlusten auf, erhält man einen Datenwürfel (2-D-Abbildung + EELS-Spektrum). Die Energieaufösung ist in diesem Fall abhängig von der Breite des gewählten Energiebereiches. Im Vergleich zu einem direkt aufgenommenen EELS-Spektrum ist die Energieauflösung jedoch deutlich reduziert. Wenn die in der Probe enthaltenen Elemente bereits bekannt sind und das Ziel der Untersuchung die Elementverteilung ist, ist es in der Regel besser, statt eines kompletten Datenwürfels, eine Elementverteilungskarte mittels EFTEM herzustellen. Dazu werden bei der Drei-Kanten-Methode zwei Abbildungen vor der Absorptionskante des zu messenden Elementes und eine Abbildung direkt auf der Kante gemessen. Durch die zwei Abbildungen vor der Kante ist es möglich, eine zuverlässige Hintergrundkorrektur des gemessenen Elementes durchzuführen. Somit sind für jedes Element nur drei Abbildungen mit relativ großem Energiebereich (z.B. $30 \mathrm{eV}$ ) nötig, was im Vergleich zur Elementverteilungskarte im STEM-Modus den Vorteil der geringeren Messzeit und damit der geringeren Elektronendosis hat, was wiederum die Probe schont.

Ein auch in dieser Arbeit benutztes EFTEM-Verfahren dient der Bestimmung der relativen Dicke der Probe. Bei der sogenannten „Log-Ratio“-Methode wird je eine EFTEM-Abbildung im Energiebereich des „Zero-Loss“-Peaks ${ }^{1}$ und

\footnotetext{
${ }^{1}$ Der ,Zero-Loss“ Peak entsteht durch Strahlelektronen, die nicht von der Probe beeinflusst wurden.
} 
eine im sich anschließenden „low-loss“ Bereich erstellt². Über die Formel

$$
\frac{t}{\lambda}=\ln \left(\frac{I_{p}}{I_{0}}\right)
$$

sind diese Abbildungen mit der relativen Dicke verknüpft. Dabei ist $I_{0}$ die Fläche unter dem ,zero-loss“-Peak und $I_{p}$ die Fläche unter dem Bereich des „low-loss“-Spektrums, $t$ ist die absolute Dicke der Probe und $\lambda$ die absolute, mittlere freie Weglänge für inelastisch gestreute Elektronen. Stellt man nun den rechten Gleichungsteil von 3.7 in einer Abbildung dar, entspricht die Helligkeit der Abbildung der relativen Dicke der Probe (s. Abb. 3.5.)

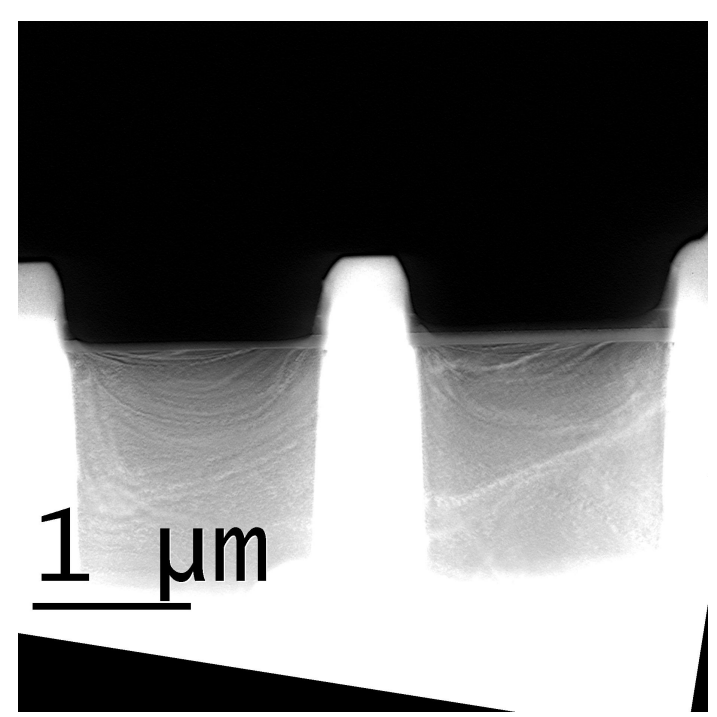

Abbildung 3.5.: Relative Dickenverteilungskarte einer FIB-TEM-Probe. Gut sichtbar der dicke mittlere Steg und die Zunahme der Dicke zum unteren Ende der Lamelle.

In homogenen Materialien ist somit eine gute Aussage über die Dickenvariation gegeben. Die relative Dicke über eine Materialgrenzfläche hinweg ist von nur geringer Aussagekraft, da sich hier die mittlere freie Weglänge $\lambda$ der Elektronen ändert. Damit ist es möglich, dass sich die relative Dicke über eine solche Grenzfläche ändert, die absolute Dicke jedoch konstant bleibt. Diese Methode zur Messung der Dickenverteilung wurde im Wesentlichen zur Dickenkontrolle nach dem Nachdünnen der TEM-Lamellen (s. Abschnitt 3.4 genutzt, bot jedoch beim Vergleich mit simulierten TEM-Abbildungen auch einen guten

\footnotetext{
${ }^{2}$ Dieser Bereich ist gekennzeichnet durch den Plasmonen-Peak, der von Strahlelektronen herrührt, die nur durch Probenplasmonen beeinflusst wurden.
} 
Anhaltspunkt für die absolute Dicke der Probe. Der Fehler der „Log-Ratio“Methode zur Bestimmung der relativen Dicke liegt für Proben bis $t / \lambda \leq 4$ bei etwa $10 \%$ [86, 87]. Je nach Herkunft der mittleren freien Weglänge zur Bestimmung der absoluten Dicke reduziert sich die Genauigkeit der Messung deutlich.

\subsubsection{Vor- und Nachteile von EELS gegenüber EDX}

Da jede Methode ihre spezifischen Vor- und Nachteile aufweist, gilt es je nach Ziel einer Untersuchung abzuwägen, ob EDX oder EELS für eine Analyse genutzt wird. In der Tabelle 3.1 sind die Vorteile der jeweiligen Techniken bei gleichen Abbildungsbedingungen dargestellt:

\begin{tabular}{|l|l|}
\hline Vorteile von EELS & Vorteile von EDX \\
\hline \hline stärkeres Signal & geringerer spektraler Hintergrund \\
\hline größere räumliche Auflösung & benötigt keine dünnen Proben \\
\hline $\begin{array}{l}\text { absolute, detektorunabhängige } \\
\text { Quantifizierung }\end{array}$ & einfachere Nutzung \\
\hline liefert Strukturinformationen & weitere Verbreitung \\
\hline
\end{tabular}

Tabelle 3.1.: Vorteile von EELS und EDX bei gleichen Abbildungsbedingungen. Nach 84

Als Vergleich dient in dieser Tabelle ein fensterloses EDX-System. EDXDetektoren, die im Philips CM200 und im FEI Titan zum Einsatz kommen, sind jedoch mit einer ultradünnen Polymerfolie vor Verunreinigungen geschützt. Die in dieser Folie stattfindende Absorption führt in EDX-Systemen zusammen mit einer reduzierten Detektoreffizienz bei kleinen Energien der Röntgenquanten zu einer schlechten Detektierung von leichten Elementen. Eine noch höhere Sensibilität für leichte Elemente als mit fensterlosen EDX-Detektoren erreicht man mit EELS, welche von besonderer Bedeutung bei der Untersuchung der Borverteilung in MTJs in Abschnitt 4.2 ist [88]. Bei EELS ist ebenfalls die hohe räumliche Auflösung bei der Interpretation der Ergebnisse bei Untersuchungen von nur $2 \mathrm{~nm}$ dicken $\mathrm{MgO}$-Schichten vorteilhaft. Diese höhere räumliche Auflösung beim EELS resultiert aus der Begrenzung der Strahlaufweitung aufgrund von elastischer Streuung durch Einsatz einer Blende. Des Weiteren können mithilfe der EELS-Spektren wichtige Strukturinformationen gewonnen werden. Die Untersuchungen der Elektromigration in Abschnitt 4.3, in der Elementverteilungskarten schwererer Elemente erstellt wurden, sind dagegen mit der EDX-Methode erstellt worden. Hier wurde auch keine hohe räumliche Auflösung benötigt. 


\subsection{IDISMA}

In Folge der Miniaturisierung von (Halbleiter-) Bauelementen kommt der Grenzfläche zwischen kristallinen und amorphen Materialien eine immer wichtigere Bedeutung zu. In dieser Arbeit wird die Grenzfläche zwischen kristallinem $\mathrm{MgO}$ und amorphem $\mathrm{CoFeB}$, wie sie auch in Tunnelbarrieren und den ferromagnetischen Elektroden von MTJs vorkommt, untersucht. Wie bereits Abschnitt 2.2 zeigte, hat diese Grenzfläche einen entscheidenen Einfluss auf den TMR in diesen Systemen. So sollen bereits wenige Monolagen kristallinen Eisens an der Grenzfläche ausreichen, um mit vollständig kristallinen Elektroden vergleichbare TMR zu erzielen [11, 44].

Für die Untersuchung der Struktur innerer Grenzflächen in Kristallen ist die hochauflösende Transmissionselektronenmikroskopie eine häufig eingesetzte Methode. Da in diesen Systemen die jeweiligen Atompositionen bekannt sind, kann hier ein direkter quantitativer Vergleich zwischen simulierten und experimentellen Abbildungen durchgeführt werden. Bei Grenzflächen zwischen kristallinen und amorphen Materialien ist ein direkter quantitativer Vergleich von experimentellen und simulierten Abbildungen jedoch aufgrund der Vielzahl möglicher struktureller Realisierungen des Übergangsbereiches nicht möglich.

Bei der kristallin/amorphen Grenzfläche handelt es sich um ein System, in dem ein Übergang von einem System mit Fernordnung in ein System mit Nahordnung bzw. mittelreichweitiger Ordnung stattfindet. Der kristalline Teil kann durch eine Fernordnung beschrieben werden, also eine Ordnung, die, verglichen mit der Gitterkonstanten, über größere Distanzen vorhanden ist. Der amorphe Teil wird durch eine Nahordnung bzw. mittelreichweitige Ordnung beschrieben, bei der die Korrelation von Atomen auf Nachbarplätzen mit dessen Abstand stark abnimmt, also typischerweise auf $<0,5 \mathrm{~nm}$. Diese Ordnung in amorphen Systemen kann nur mittels statistischer Methoden beschrieben werden. Hierzu dient u.a. die Paarverteilungsfunktion, welche die Häufigkeit angibt, ein Atom in einem bestimmten Abstand anzutreffen. Sie kann z.B. mittels Beugungsmethoden gemessen werden. In kristallinen Systemen ist hingegen eine genaue Angabe der Atompositionen möglich. An der Grenzfläche von kristallinem und amorphem Material kommt es jedoch zu einer Bindung zwischen Atomen beider Materialien und einer induzierten Ordnung in einem Übergangsbereich des amorphen Materials

Die hier durchgeführte Untersuchung der c-MgO/a-CoFeB-Grenzfläche ba-

\footnotetext{
${ }^{6}$ Auch eine Beeinflussung des Kristalls durch das amorphe Material ist möglich, wird hier aber vernachlässigt.
} 
siert auf dem IDIM-Verfahren („Iterative Digital Image Matching“) von Möbus [89, 90], das eine iterative Anpassung einzelner simulierter HRTEM-Abbildungen an experimentellen Abbildungen beschreibt. Dieses Verfahren wurde von Borgardt et al. [13, 51, 14] zum einen um die simultane Anpassung ganzer Abbildungsserien erweitert (IDISMA - Iterative Digital Image Series Matching) und zum anderen um die APP-Methode (siehe Abschnitt 3.3.3) zur Simulation von amorph/kristallinen-Grenzflächen ergänzt. Diese Kombination der IDISMA- und der APP-Methode wurde u.a. von Thiel et al. [53, 21] für die Untersuchung der a-Si/c-Si und der a-Ge/c-Si Grenzfläche genutzt.

Durch die Verwendung mehrerer Abbildungen, welche unter unterschiedlichen Defoki aufgenommen worden sind, ist eine gute Trennung der grenzflächeninduzierten Ordnung von Delokalisierungseffekten gegeben. Außerdem erhöht sich bei der Verwendung von Abbildungsserien die Signifikanz der angepassten Strukturparameter gegenüber nur einer angepassten Abbildung.

Das Grundprinzip der hier verwendeten IDISMA-Methode soll in den folgenden Abschnitten genauer erläutert werden. Da diese Methode mit experimentellen Defokusserienabbildungen arbeitet, die immer einer gewissen Probendrift unterliegen, müssen diese zuerst durch eine Driftkorrektur korrigiert werden. Diese Korrektur sowie weitere, für einen quantitativen Vergleich notwendige Maßnahmen, werden in Abschnitt 3.3.1 näher beschrieben.

Die Simulationen der elektronenmikroskopischen Abbildungen auf Basis eines Strukturmodells werden mittels der bekannten Multislice-Methode [20, 16, 17, 18, 19] erstellt. Hierfür wird in dieser Arbeit das EMS-Programmpaket von Stadelmann [91] genutzt, das sich gut in einen iterativen Prozess integrieren lässt. In Abschnitt 3.3.2 wird eine kurze Einführung in die Multislice-Methode zur Simulation von HRTEM-Abbildungen gegeben.

Um die HRTEM-Abbildungen der amorph/kristallinen Grenzfläche mittels Multislice-Methode erstellen zu können, wird in Abschnitt 3.3.3 die „AveragedProjected-Potential"-Näherung (APP-Näherung) vorgestellt.

Für die Bildung des mittleren projizierten Potentials der Grenzfläche zwischen kristallinem $\mathrm{MgO}$ und amorphem $\mathrm{CoFeB}$ dient ein parametrisiertes Strukturmodell, dessen Konstruktion in Abschnitt 3.3 .3 näher erläutert wird.

Im Anschluss an die Simulation findet ein Vergleich der Defokusserie der simulierten und experimentellen Abbildungen statt, dessen Funktionsweise in Abschnitt 3.3.5 behandelt wird.

Durch Änderungen der in das Strukturmodell einfließenden Parameter und erneutem Vergleich der experimentellen und simulierten Abbildungen wird in einem iterativen Prozess der Kontrastunterschied zwischen diesen Abbildungen minimiert. Als Ergebnis des iterativen Optimierungsprozesses erhält man 
direkt eine mögliche strukturelle Realisierung der Grenzfläche, was bei der in Abschnitt 3.1.3 vorgestellten Objektwellenrekonstruktion nicht direkt der Fall ist. Durch die Bestimmung der strukturellen Ordnung an der Grenzfläche ist eine Ausdehnungsquantifizierung des durch das $\mathrm{MgO}$ beeinflussten Übergangsbereiches möglich.

Einige der in Abschnitt 3.3 verwendeten Prozesse (z.B. die Mittelung entlang der Grenzfläche) und Methoden (APP-Näherung) wurden von Borgardt et al. [13, 51, 14, Plikat [22, 52], Thiel [21, 53] und Eilers et al. [92] entwickelt bzw. angewendet. Zur besseren Lesbarkeit wird im folgenden Abschnitt auf die Einzelverweise verzichtet.

\subsubsection{Driftkorrektur, Mittelung und Bestimmung von Simulationsparametern}

Bevor die experimentellen Abbildungen mit den simulierten Daten verglichen werden können, müssen die experimentellen Daten entsprechend aufbereitet werden. Hierfür ist es grundsätzlich notwendig, eine Driftkorrektur durchzuführen, damit der untersuchte Probenbereich räumlich immer der gleiche ist. Dieser Schritt ist erforderlich, da die Probe im TEM während der Aufnahme der Serie üblicherweise um wenige $\mathrm{nm}$ driftet. Diese Drift ist insbesondere während der Belichtung der CCD-Kamera kritisch, da hierdurch die Abbildung verschmiert. Die hier verwendete Belichtungszeit ist mit 0,5 s jedoch hinreichend kurz, um keine Effekte der Probendrift auf der Aufnahme festzustellen. Der Großteil der Probendrift tritt in der Zeit zwischen den Aufnahmen auf, da hier die Zeitspanne mit 5 s deutlich größer ist. Diese Zeitspanne wurde gewählt, damit sich das Gerät nach der Veränderung des Fokus stabilisieren kann und keine Fokusschwankungen auftreten. Die Driftkorrektur erfolgt mit TrueImage Professional 2.0.1 (Build 7) von FEI mittels eines Kreuzkorrelationsverfahrens, das nur auf die niederfrequenten Anteile des Bildsignals angewendet wird. Durch den Tiefpassfilter wird die Driftkorrektur nur auf die nicht periodischen Strukturen angewendet. Dadurch ist es möglich, die Abbildungen trotz unterschiedlicher Abbildungsbedingungen präzise auszurichten [93. Da die hier auftretende absolute Drift ${ }^{7}$ von $0,67 \mathrm{~nm}$ (s. Abb. 3.6) über die ganze Defokusserie sehr gering ist, kann der Nebeneffekt der Reduktion des Bildfeldes durch die Driftkorrektur auf dem Bildbereich, der in allen Abbildungen der Defokusserie enthalten ist, vernachlässigt werden. Die mittlere Drift zwischen zwei Abbildungen beträgt $0.04 \mathrm{~nm}$. Somit tritt während der Belichtung der Aufnahme eine errechnete, mittlere Drift von $<0.004 \mathrm{~nm}$ auf, was weit unter

${ }^{7}$ Drift zwischen der ersten und der letzten Abbildung einer Serie. 
dem Informationslimit des verwendeten Mikroskops liegt.

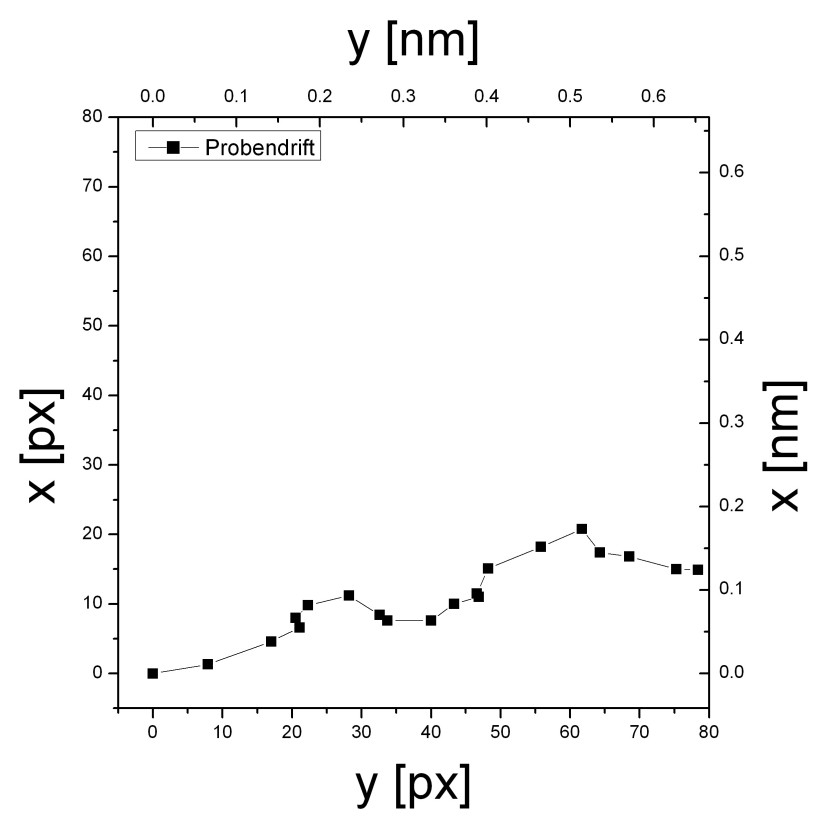

Abbildung 3.6.: Gemessener absoluter Driftverlauf der Probe in Pixeln und nm. Der Hauptanteil der Drift tritt entlang der $y$-Achse auf, was auf eine nach einer Bewegung nicht vollständig zur Ruhe gekommene Stage zurückzuführen ist, da die yAchse hier nahezu parallel zur Grenzfläche verläuft. Die gesamte Drift ist mit 0,67 nm über die ganze Messdauer sehr gering. Die maximale Drift zwischen zwei Abbildungen ist mit $0.08 \mathrm{~nm}$ zwischen Abbildung 2 und 3 ebenfalls sehr gering.

Um aus den experimentellen Abbildungen die kristallperiodische Struktur zwischen dem hier untersuchten kristallinen $\mathrm{MgO}$ und dem amorphen $\mathrm{CoFeB}$ zu extrahieren, wurde eine periodische Mittelung mit der Streifenbreite d entlang der Grenzfläche durchgeführt. Das führt zu einer Reduktion auf die dperiodische Informationen an der Grenzfläche. Durch diese Mittelung ist eine statistische Beschreibung der grenzflächeninduzierten Ordnung des amorphen Materials anhand einer Verteilungsfunktion im Rahmen der APP-Näherung (s. Abschnitt 3.3.3 erst sinnvoll.

Die Probe wird zunächst, wie in Abbildung 3.7c ersichtlich, entlang der $\mathrm{MgO}[100]-Z o n e n a c h s e$ orientiert und durchstrahlt. Dabei liegt die Grenzfläche 




(a)

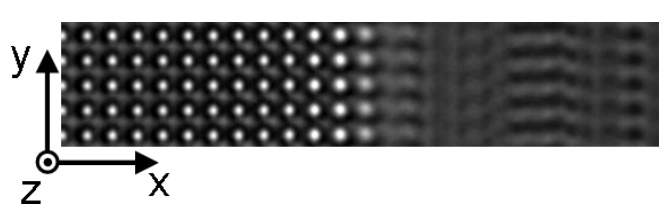

(b)

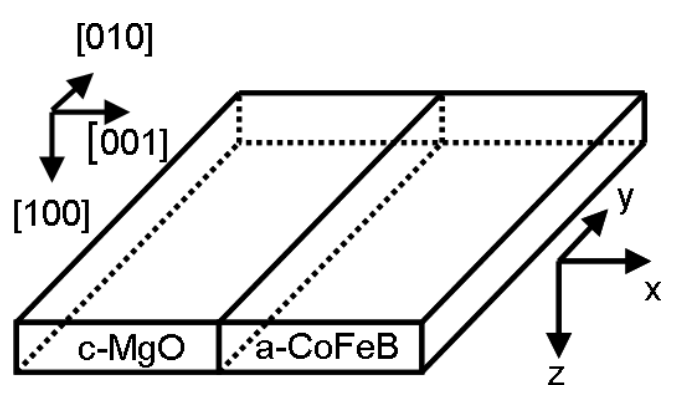

(c)

Abbildung 3.7.: a) Abbildung der c-MgO/a-CoFeB-Grenzfläche bei einem Defokus von $\Delta f=1 \mathrm{~nm}$ mit Einteilung in einzelne Streifen und der Periode d. b) Gemittelte Grenzflächenabbildung nach 3.8 , vierfach entlang der y-Achse wiederholt. c) Skizze der c-MgO/a-CoFeB-Grenzfläche mit eingezeichneter Kristallorientierung. Die $x$-Achse über die Grenzfläche hinweg ist die MgO[001]-Richtung, parallel zur Grenzfläche verläuft die y-Richtung, welche der MgO[010]-Richtung entspricht. Die Probennormale z verläuft entlang der MgO[100]-Richtung. Nach [45].

zum amorphen $\mathrm{CoFeB}$ parallel zur $\mathrm{MgO}[010]-R i c h t u n g$. Für die Mittelung werden die ausgerichteten Abbildungen der Probe in Streifen parallel zur Grenzfläche eingeteilt (s. Abb. 3.7a). Die Streifenbreite entspricht dabei genau dem halben Gitterabstand des $\mathrm{MgO} d=1 / 2 a_{M g O}=0,21 \mathrm{~nm}$, die der Periodizität im kristallinen Teil der Probe entspricht. Anschließend werden die Streifen über alle äquivalenten Punkte gemittelt:

$$
I^{\prime}(x, y)=\frac{s_{d}(y)}{N} \sum_{n=-(N-1) / 2}^{(N-1) / 2} I(x, y+n d)
$$

$I^{\prime}(x, y)$ bezeichnet dabei die gemittelte Intensitätsverteilung, $I(x, y)$ die Intensitätsverteilung in der experimentellen Abbildung am Punkt $(x, y), N$ die Anzahl der Streifen und $s_{d}$ ist die Spaltfunktion:

$$
s_{d}(x)= \begin{cases}1: & -\frac{d}{2} \leq y \leq \frac{d}{2} \\ 0: & \text { sonst }\end{cases}
$$

Eine aus dieser Mittelung hervorgehende Grenzflächenabbildung ist in Ab- 
bildung $3.7 \mathrm{~b}$ zu sehen. Sie ist hier zur besseren Visualisierung fünffach untereinander fortgesetzt dargestellt.

In dieser Arbeit finden sich auch eindimensionale Intensitätsprofile, die sich aus der Integration entlang der y-Richtung des Streifens ergeben. Diese Profile dienen nur der Visualisierung; die quantitativen Vergleiche werden ausschließlich mit den gemittelten zweidimensionalen Abbildungen durchgeführt.

Beim direkten Vergleich simulierter und experimenteller Abbildungen tritt ein systematischer Unterschied in den Kontrasten auf, weshalb ein quantitativer Vergleich nicht problemlos möglich ist. Diese Diskrepanz ist in der Literatur als „Stobbs-Faktor" bekannt [94, 95]. Die Ursachen für die auftretenden Unterschiede sind noch immer Gegenstand der Forschung [58, 96, 97, 98, 99] und liegen vermutlich in der Kombination verschiedener Effekte, die in der Simulation nicht berücksichtigt werden. Aufgrund dieser Kontrastunterschiede von simulierten und experimentellen Abbildungen müssen die Abbildungen vor einem quantitativen Vergleich normiert werden. Bei dem von Möbus [89] vorgestellten Verfahren werden die experimentellen Abbildungen auf die mittlere Gesamtintensität des Elektronenstrahls normiert. Zu diesem Zweck wurde aus einer Abbildung ohne Probe die mittlere Intensität $<I_{\text {Ref }}>$ bestimmt und die Proben wie folgt normiert:

$$
I_{\text {norm }}(x, y)=\frac{I(x, y)}{<I_{\text {Ref }}>}
$$

Für die Simulation der elektronenmikroskopischen Abbildungen werden außerdem die Abbildungsparameter wie der Startdefokus und die Defokusschrittweite benötigt. Außerdem ist die Dicke der Proben für die Simulation zwingend erforderlich. Diese Simulationsparameter werden hier ebenfalls in einem iterativen Prozess bestimmt, in dem der kristalline Bereich der experimentellen Abbildungen mit simulierten Abbildungen verglichen wird. Um den Prozess zu verkürzen, ist es angebracht den Parameterraum einzugrenzen.

Da im hier verwendeten FEI TITAN ETEM der Defokus über dem minimalen Kontrast im Amorphen bei $\Delta f=0 \mathrm{~nm}$ gut abgeschätzt werden kann, ergibt sich hieraus ein guter Anhaltspunkt für den Startdefokus. Hierbei soll $\Delta f=0 \mathrm{~nm}$ in der Mitte der Defokusserie liegen. Aus der Objektwellenrekonstruktion von Defokusserien ist außerdem bekannt, dass die angegebene Fokusschrittweite am TEM ebenfalls sehr zuverlässig ist. Der Ort der Defokusserienaufnahme liegt lediglich wenige nm von der Probenkante entfernt, weshalb davon ausgegangen wurde, dass die Probendicke am Ort der Grenzfläche unter 20-30 nm liegt. Diese Abschätzung wurde durch eine EFTEM- 
Dickenverteilungskarte bestätigt. Die iterative Bestimmung dieser Parameter durch Vergleich des kristallinen Teils der Abbildungsserie mit simulierten Kristallabbildungen ergab hieraus einen Startdefokus von $\Delta f=28 \mathrm{~nm}$, eine Fokusschrittweite von $\delta \Delta f=-3,0 \mathrm{~nm}$ sowie eine Probendicke von $t=10,9 \mathrm{~nm}$.

\subsubsection{Multislice-Simulation von HRTEM-Abbildungen}

Die eigentliche Simulation der elektronenmikroskopischen Abbildungen wird mit der etablierten Multislice-Methode [20, 16, 17, 18, 19] durchgeführt. Dabei wird die Probe in dünne Scheiben senkrecht zum einfallenden Elektronenstrahl aufgeteilt. Die Berechnung des Streuprozesses wird als eine Folge von Streuungen an den einzelnen Scheiben beschrieben. Der einzelne Streuprozess an einer Scheibe kann in zwei Teile eingeteilt werden: Die Streuung am projizierten, zweidimensionalen Streupotential der Scheibe und die anschließende Propagation der gestreuten Welle zur nächsten Scheibe.

Zur Streuung wird das projizierte Potential $\varphi^{j}(x, y)$ genutzt, das man aus der Projektion des dreidimensionalen Streupotentials auf die zweidimensionale Eintrittsfläche der $j$-ten Scheibe erhält:

$$
\varphi^{j}(x, y)=\frac{1}{\Delta z} \int_{z_{j-1}}^{z_{j}} V(\vec{r}) d z
$$

Dabei ist $\Delta z=z_{j}-z_{j-1}$.

Bei der Streuung der Elektronenwelle am projizierten Potential erfährt diese eine Phasenverschiebung, die proportional zum projizierten Potential ist und beschrieben wird durch die Transmissionsfunktion $P^{(j)}(x, y)$. Für diese Streuung muss die Phasennäherung erfüllt sein, d.h. die Scheibe muss hinreichend dünn sein, sodass die einfallende Elektronenwelle beim Durchlaufen der Scheibe relativ zu einer Elektronenwelle im Vakuum nur eine Änderung der Phase erfährt.

$$
P^{(j)}(x, y)=\exp \left(i \sigma \varphi^{(j)}(x, y)\right)
$$

$\sigma$ ist die Wechselwirkungskonstante, welche gegeben ist durch:

$$
\sigma=\frac{\pi}{\lambda U_{R}}
$$

Dabei ist $\lambda$ die Wellenlänge der einfallenden Elektronenwelle und $U_{R}$ die relativistisch korrigierte Beschleunigungsspannung. 
Nach der Streuung am projizierten Potential breitet sich die gestreute Elektronenwelle über die Strecke $\Delta z$ durch den freien Raum zur nächsten Scheibe aus. Diese Propagation kann durch eine Faltung der Wellenfunktion $\psi^{j}(x, y)$ mit dem Fresnelpropagator $F R_{\Delta Z}(x, y)$ beschrieben werden.

$$
\psi^{j+1}(x, y)=\left[P(x, y) \cdot \psi^{(j)}(x, y)\right] \otimes F R_{\Delta Z}(x, y)
$$

mit

$$
F R_{\Delta Z}(x, y)=\frac{1}{i \lambda \Delta z} \exp \left[\frac{i \pi}{\lambda \Delta z}\left(x^{2}+y^{2}\right)\right]
$$

als Fresnelpropagator.

Bei periodischen Strukturen sind die projizierten Potentiale für alle $j$ identisch. Ebenso sind auch die Transmissionsfunktionen für alle Streifen identisch und es kann auf den Index $j$ verzichtet werden.

\subsubsection{Die „Averaged-Projected-Potential“-Näherung}

Zur Simulation von Grenzflächenabbildungen ist der atomistische Ansatz, bei dem exakte Atompositionen vorgegeben werden, aufgrund der großen Anzahl möglicher struktureller Realisierungen der amorphen Struktur nicht praktikabel.

Der hier verwendete Ansatz geht auf ein von Borgardt et al. [13] vorgeschlagenes Verfahren zurück. Bei der Methode der "Averaged-Projected-Potential“ -Näherung (APP-Näherung) wird für die Berechnung der elektronenmikroskopischen Abbildungen mittels Multislice-Verfahren anstelle des projizierten Potentials konkreter Atompositionen das mittlere, projizierte Potential $\psi^{A P P}(x, y)$ genutzt: Hierbei fließt anstelle konkreter Atompositionen eine mittlere, zweidimensionale Dichtefunktion $\rho(x, y)$ innerhalb des Streifens ein. Die Streifenbreite besitzt die gleiche Weite $d$ wie die Periodizität auf der kristallinen Seite mit der auch die experimentellen Abbildungen gemittelt wurden ( $\mathrm{s}$. Abschnitt 3.3.1.

Das mittlere, projizierte Potential ist nach Borgardt et al. [13] gegeben 
durch:

$$
\begin{array}{r}
\psi^{A P P}(x, y)=\frac{s_{d}(y)}{\Delta z} \sum_{n=-(N-1) / 2_{z_{j-1}}} \int^{(N-1) / 2} \tilde{V}\left(p_{x}, \frac{n}{d}, 0\right) \tilde{\rho}\left(p_{x}, \frac{n}{d}\right) \\
\times \exp \left[2 \pi i\left(p_{x} x+\frac{n}{d} y\right)\right] d p_{x}
\end{array}
$$

Hierbei kennzeichnen $\tilde{V}\left(p_{x}, p_{y}\right)$ und $\tilde{\rho}\left(p_{x}, p_{y}\right)$ die Fouriertransformierten des Streupotentials $V(x, y)$ und der mittleren, zweidimensionalen atomaren Dichtefunktion $\rho(x, y)$.

$s_{d}(y)$ ist die gleiche Spaltfunktion wie in Abschnitt 3.3.1.

$$
s_{d}(x)= \begin{cases}1: & -\frac{d}{2} \leq y \leq \frac{d}{2} \\ 0: & \text { sonst }\end{cases}
$$

Die mittlere, zweidimensionale Dichtefunktion $\rho(x, y)$ ist dabei die Projektion der dreidimensionalen, atomaren Dichtefunktion auf die zweidimensionale Eintrittsfläche der $j$-ten Scheibe. Die Bildung der dreidimensionalen, atomaren Dichtefunktion wird in Abschnitt 3.3.4 beschrieben.

Mit der APP-Methode kann man folglich mittels Multislice-Simulation Grenzflächenabbildungen erstellen, die auf der kristallinen Seite auf Grundlage von exakten Atompositionen und auf der amorphen Probenseite auf Grundlage des mittleren, projizierten Potentials bestehen. Somit wird es möglich, diese simulierten Grenzflächenabbildungen mit gemittelten, experimentellen Grenzflächenabbildungen zu vergleichen.

\subsubsection{Die Verteilungsfunktion für die $\mathrm{c}-\mathrm{MgO} / \mathrm{a}-\mathrm{CoFeB}$ Grenzfläche}

Die Konstruktion der dreidimensionalen Verteilungsfunktion muss im Fall der c-MgO/a-CoFeB-Grenzfläche im Vergleich zu der bei Thiel und Borgardt et al. [21, 13] verwendeten Konstruktionsvorschrift in einigen Punkten abgeändert werden. Grundsätzlich wird jedoch weiterhin davon ausgegangen, dass die amorphe Struktur keinen Einfluss auf die kristalline Struktur ausübt ${ }^{1}$. Somit bleiben die exakten Atompositionen auf der kristallinen Seite vollständig erhalten und werden nicht verzerrt. Ausgehend von der letzten konkreten Atomposition im Kristallinen wird die Struktur der Grenzfläche rekursiv von Atomlage

\footnotetext{
${ }^{1}$ Diese Annahme geht auf Beobachtungen zurück, welche bei HRTEM-Untersuchungen der grenzflächeninduzierten Ordnung an der fest/flüssig-Grenzfläche von Howe [100, gemacht wurden.
} 
zu Atomlage in das amorphe Material hinein fortgesetzt. Jedoch wird bei diesem System angenommen, dass die atomare Dichte im Amorphen gaussförmig um diese Position verteilt ist. Dabei nimmt die Standardabweichung $\sigma$ mit den weiteren Atomlagen $i$ zu, sodass die Verteilung der atomaren Dichte mit steigendem Abstand zur Grenzfläche immer homogener wird (s. Skizze 3.9a). Der Grund hierfür ist, dass das hier untersuchte amorphe CoFeB einen metallischen Bindungscharakter aufweist, der keine gerichteten Verbindungen wie das bei Thiel [21] untersuchte kovalente Bindungssystem in amorphem Germanium oder amorphem Silizium besitzt.

Ausgehend von der Annahme, dass die mittlere Atomdichte $f_{i-1}(r)$ in der $i$-1-ten Lage bekannt ist, ergibt sich die mittlere Atomdichte $f_{i}(r)$ mit

$$
f_{i}(r)=f_{i-1}(r) * W_{i}(r)
$$

Dabei ist $W_{i}(r)$ die Antwortfunktion, die die Konstruktion der Grenzfläche beschreibt. Wie bereits oben beschrieben, wird die atomare Dichte im Amorphen gaussförmig um eine Position verteilt:

$$
W_{i}(x, y, z)=\left(\frac{1}{2 \pi}\right)^{3 / 2} \frac{1}{\sigma_{x i} \sigma_{y i} \sigma_{z i}} e^{-\frac{\left(x-x_{0 i}\right)^{2}}{2 \sigma_{x i}^{2}}} e^{-\frac{\left(y-y_{0 i}\right)^{2}}{2 \sigma_{y i}^{2}}} e^{-\frac{\left(z-z_{0 i}\right)^{2}}{2 \sigma_{z i}^{2}}}
$$

Diese Konstruktion unterliegt der Annahme, dass die Standardabweichung in alle Richtungen parallel zur Grenzfläche gleich ist: $\sigma_{y i}=\sigma_{z i}$. Für den ersten Abstand zwischen dem letzten MgO-Sauerstoff-Atom und dem ersten Fe-Atom gilt: $x_{x 1}=d_{a 1}$ und für alle folgenden Atomlagen gilt $x_{x i}=d_{a 2}$ für $i \geq 2$. Diese Annahme für $i \geq 2$ wird aus Vereinfachungsgründen durchgeführt und ist dadurch berechtigt, dass die nächsten Nachbarn eines Fe-Atoms in der $i \geq 2$ Lage für alle Fe-Atome für $i \geq 2$ identisch sind.

Außerdem gilt, wie in Abb. 3.8 ersichtlich: $y_{01}=0$ und $z_{01}=0$ sowie $y_{0 i}=$ $1 / 2 a_{M g O}$ und $z_{0 i}=i \cdot 1 / 2 a_{M g O}$ für $i \geq 2$. Das heißt, dass die erste Lage Eisen senkrecht im Abstand $d_{a 1}$ auf dem letzten Sauerstoffatom vom MgO steht. Das $\sigma_{y z 1}$ und $\sigma_{x 1}$ wird unabhängig von den weiteren Lagen bestimmt. Für $i>2$ wird $\sigma_{y z i}, \sigma_{x i}$ durch den Parameter $a_{x}$ bzw. $a_{y z}$ modifiziert. $a_{x}$ bzw. $a_{y z}$ gibt die Zunahme von $\sigma_{y z 2}$ und $\sigma_{x 2}$ basierend auf den Anfangswerten $\sigma_{y z 2}$ und $\sigma_{x 2}$ für die kommenden Lagen an, wie dies auch in der Abbildung 3.9 b dargestellt ist. Somit ergibt sich:

$$
\sigma_{x i}=\sigma_{x 2}+(i-2) \cdot a_{x} \quad \text { mit } \quad i \in \mathbb{Z} \mid i>2
$$

bzw.

$$
\sigma_{y z i}=\sigma_{y z 2}+(i-2) \cdot a_{y z} \quad \text { mit } \quad i \in \mathbb{Z} \mid i>2
$$


Aus diesem Modell ergeben sich insgesamt acht Parameter, um die dreidimensionale Verteilungsfunktion an der Grenzfläche zwischen c-MgO und aCoFeB vollständig zu beschreiben: $d_{a 1}, d_{a 2}, \sigma_{x 1}, \sigma_{y z 1}, \sigma_{x 2}, \sigma_{y z 2}, a_{x}, a_{y z}$. Diese acht Parameter werden im Rahmen der IDISMA-Methode iterativ bestimmt.

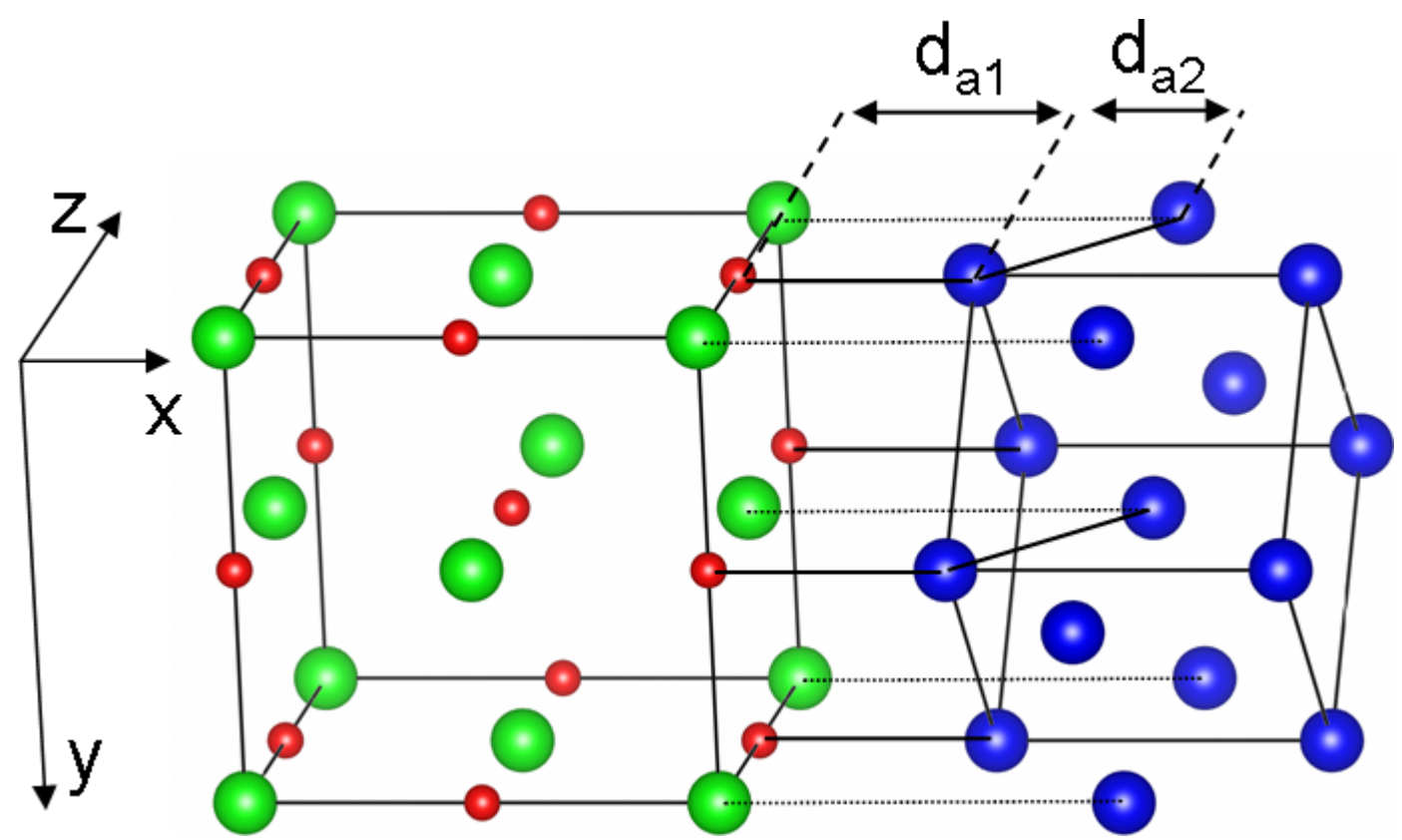

Abbildung 3.8.: Lage der Erwartungswerte für die dreidimensionale Verteilungsfunktion $\rho_{3 D}(r)$ für die ersten drei Atomlagen. Die Blickrichtung im TEM ist entlang der z-Achse.

Die dreidimensionale Atomdichte $\rho_{3 D}(r)$ im Amorphen ergibt sich aus der Summe über alle mittleren Atomdichten $f_{i}(r)$ in allen Atomlagen $i$. Die Berechnung findet innerhalb einer Zelle mit den Abmessungen $b=c=a_{M g O}$ und $a$ statt. $a$ ist variabel und richtet sich nach der Länge des Übergangsbereiches.

Die zweidimensionalen Verteilungsfunktion $\rho(x, y)$ erhält man durch:

$$
\rho(x, y)=\int_{0}^{c} \rho_{3 D}(r) d z
$$

Aus dieser zweidimensionalen Verteilungsfunktion $\rho(x, y)$ erhält man mit Hilfe der Gleichung 3.15 das mittlere Potential. Die hier untersuchten amorphen Schichten bestehen aus einer $\mathrm{Co}_{20} \mathrm{Fe}_{60} \mathrm{~B}_{20}$-Legierung. Sie enthält also weitaus mehr Eisen als Cobalt. Der Unterschied im Streupotential von Cobalt 
a)

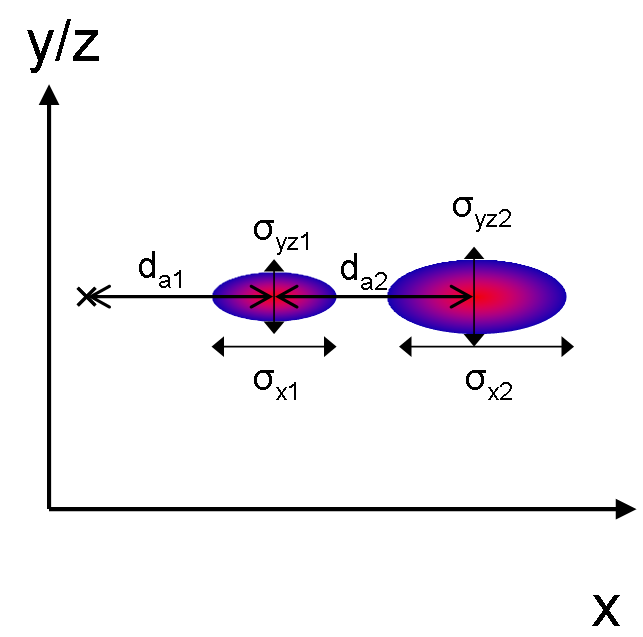

b)

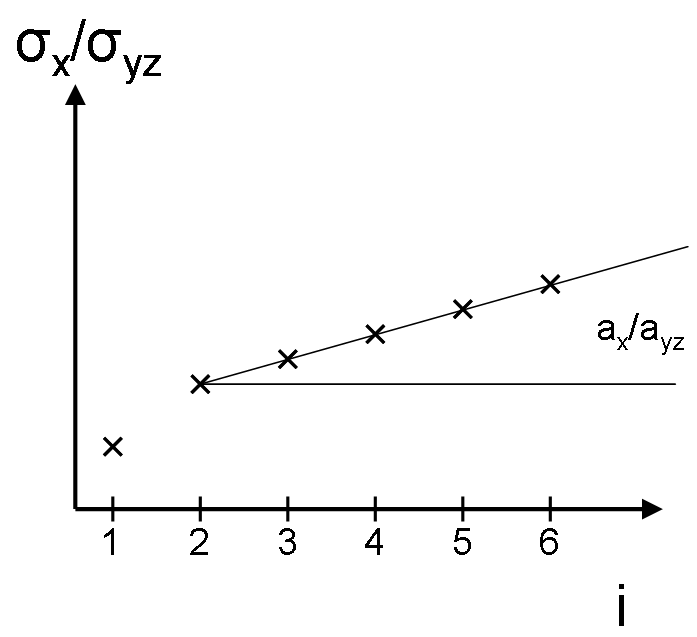

Abbildung 3.9.: Konstruktion der dreidimensionalen Verteilungsfunktion: a) Veranschaulichung der Parameter $d_{a i}$ und $\left.\sigma_{x i / y z i} b\right)$ Einfluss der Parameter $a_{x / y z}$ auf $\sigma_{x / y z}$ mit steigender Atomlage $i$.

und Eisen ist, wie in Abbildung A.1 im Anhang gut ersichtlich, sehr gering. Ebenso ist hier erkennbar, dass das Bor im Vergleich zu Eisen und Cobalt nur ein geringes Streupotential aufweist und somit kaum zum Kontrast beiträgt. Außerdem ist davon auszugehen, dass sich in kristallinem CoFe sowie im Übergangsbereich zwischen c-MgO und a-CoFeB weniger Bor befindet als im a-CoFeB. Daher wird für das atomare Streupotential in diesem Fall und aufgrund von numerischen Gründen nur das atomare Streupotential von Eisen genutzt.

Von den acht angepassten Parametern lassen sich die Abstände $d_{a 1}$ und $d_{a 2}$ am leichtesten mit der Literatur vergleichen. $d_{a 1}$ bezeichnet den Abstand der ersten Atomlage des pseudomorph aufwachsenden CoFeB zum letzten Sauerstoffatom des $\mathrm{MgO}$. Literaturwerte für diesen Abstand existieren hauptsächlich von Fe-Schichten auf $\mathrm{MgO}$ und nur sehr wenige von CoFeB. Darüber hinaus sind die Fe-MgO-Grenzflächen in der Literatur zum Teil oxidiert und weisen tendenziell größere Bindungslängen auf als die nicht oxidierten.

Der Abstand $d_{a 2}$ zwischen der ersten und der zweiten Atomlage korrespondiert mit dem halben Gitterabstand von kristallinem bcc-Co $\mathrm{Fe}_{1-x}$ mit $x=0-0,7$. Dieser beträgt $1 / 2 \cdot a_{C o F e}=0,143 \mathrm{~nm}$ bei einer Gitterkonstanten von $a_{C o F e}=0,285 \mathrm{~nm}$ [41. In amorphen Systemen wird dieser Abstand durch den Nächste-Nachbar-Abstand definiert, der durch das erste Maximum 
der Paarverteilungsfunktion beschrieben wird. Es gibt die Häufigkeit an, ein Teilchen in einem bestimmten Abstand zu finden. Dieser Abstand wurde in dünnen amorphen $\mathrm{CoFeB}-S c h i c h t e n$ mittels Elektronenbeugung gemessen und beträgt $d_{a-C o F e B}=0,253 \mathrm{~nm}[101$.

\subsubsection{Vergleich experimenteller und simulierter Abbildungen}

Beim quantitativen Vergleich simulierter und experimenteller HRTEM-Abbildungen fällt ein Kontrastunterschied auf, der eine Normierung der Abbildungen notwendig macht. Einen Überblick über die verschiedenen Effekte, die einen Einfluss auf den Kontrast haben, ist bei Boothroyd [96] zu finden. Dazu zählen unter anderem Probenvibrationen, Phononenstreuung, Verpannungen der Probe und amorphe Deckschichten. Darüber hinaus hat auch das Aufzeichnungsmedium, hier eine CCD-Kamera, einen Einfluss auf die aufgezeichnete Abbildung.

Da ein CCD-Chip Elektronen nicht direkt abbildet, sitzt vor dem Chip ein Szintillator, in dem die einfallenden Elektroden Photonen erzeugen, die durch eine Glasfaseroptik auf den CCD-Chip geleitet werden. Durch Streuung der Elektronen und Photonen im Szintillator findet man das Signal eines Elektrons auf der CCD nicht in einem Pixel, sondern ebenfalls in den umliegenden Pixeln wieder. Diese gerätespezifische Eigenschaft der CCD-Kamera führt zu einer Dämpfung hoher Raumfrequenzen und somit zu einem Kontrastverlust. Diese Dämpfung wird durch die radialsymmetrische Modulationstransferfunktion (MTF) beschrieben. Um den Einfluss der CCD-Kamera beim Vergleich simulierter und experimenteller Abbildungen zu berücksichtigen, wird die MTF im Fourierraum mit den simulierten Abbildungen multipliziert. Die MTF der in dieser Arbeit verwendeten CCD-Kamera vom Typ UltraScan 1000XP der Firma Gatan wurde anhand der Kantenmethode [102] bestimmt und durch die Summe zweier Lorentz-Linien und einer Konstanten parametrisiert:

$$
\operatorname{MTF}(q)=\frac{a_{1}}{1+\beta_{1} q^{2}}+\frac{a_{2}}{1+\beta_{2} q^{2}}+c
$$

$q$ beschreibt dabei die Raumfrequenz. Die Parameter der hier verwendeten CCD-Kamera wurden wie folgt bestimmt:

Vielfach wird auch die ungenaue Bestimmung der MTF als Ursache für den „Stobbs-Faktor“ (s. Abschnitt 3.3.1] angesehen [103].

Der quantitative Bildvergleich der simulierten und experimentellen Defokusserien wurde nach der gleichen Methode wie bei Thiel [21] durchgeführt. Als 


\begin{tabular}{|c|c|}
\hline$a_{1}$ & 0,455 \\
\hline$a_{2}$ & 0,642 \\
\hline$\beta_{1}$ & 8.682 \\
\hline$\beta_{2}$ & 586.37 \\
\hline$c$ & -0.109 \\
\hline
\end{tabular}

Tabelle 3.2.: Bestimmte MTF-Parameter der verwendeten CCD-Kamera bei 300 KV Beschleunigungsspannung.

Differenzmaß dient der R-Faktor, der in ähnlicher Form auch bei der Analyse von Röntgenbeugungsmustern [89] verwendet wird:

$$
R=\frac{1}{N} \sum_{k} R_{k}\left(I_{\text {exp }}^{(k)}, I_{\text {sim }}^{(k)}\right)=\frac{1}{N} \sum_{k} \sum_{x y} \frac{\left[\left(I_{\text {exp }}^{(k)}\right)_{x y}-\left(I_{\text {sim }}^{(k)}\right)_{x y}\right]^{2}}{\left[\left(I_{\text {exp }}^{(k)}\right)_{x y}\right]^{2}}
$$

$\left(I_{\text {sim }}^{(k)}\right)_{x y}$ und $\left(I_{\text {exp }}^{(k)}\right)_{x y}$ bezeichnen dabei die Intensität der k-ten Abbildung der simulierten bzw. experimentellen Abbildungen am Ort $(x, y)$. Ein geringerer R-Faktor bedeutet, dass eine bessere Übereinstimmung zwischen den experimentellen und den simulierten Abbildungen gegeben ist als bei größeren $\mathrm{R}$. 


\subsection{Probenpräparation}

Die Untersuchung der Grenzfläche zwischen kristallinem MgO und amorphem CoFeB stellt hohe Ansprüche an die TEM-Lamelle. So sind sehr dünne Proben erforderlich, die möglichst keine Dickenvariationen über die Grenzfläche hinweg aufweisen und entlang der Grenzfläche homogen dünn sind. Dies folgt aus der Voraussetzung, dass senkrecht zur Grenzfläche gemittelt wird. Darüber hinaus sollen die Lamellen möglichst keine amorphen Deckschichten (durch Kohlenwasserstoffe oder amorphisiertes Probenmaterial) aufweisen und auch nicht durch Implantation oder Redeposition vom Probenträgermaterial verunreinigt sein.

Deshalb wurde bei der Präparation der TEM-Proben eine Kombination aus der FIB-Methode (Focused Ion Beam) und einem anschließenden Nachdünnschritt in einem „Precision Ion Polishing System “ (PIPS) der Firma Gatan angewendet. In diesem werden $\mathrm{Ar}^{+}$-Ionen mit Beschleunigungsspannungen bis hinab auf $100 \mathrm{~V}$ für den finalen Dünnschritt benutzt. Da das PIPS im Gegensatz zum FIB nicht mit einem fokussierten Ionenstrahl arbeitet, sind einige Änderungen an der FIB-Prozedur nötig. Diese Änderungen sollen eine Redeposition von Trägermaterial auf die TEM-Probe während des Nachdünnens in der PIPS minimieren. Die ursprüngliche FIB-Prozedur ist detailliert in [104] beschrieben, erfährt jedoch aufgrund der weiteren Bearbeitung der Lamelle im PIPS sowie materialspezifischer Besonderheiten einige Änderungen, die im Folgenden kurz dargestellt werden.

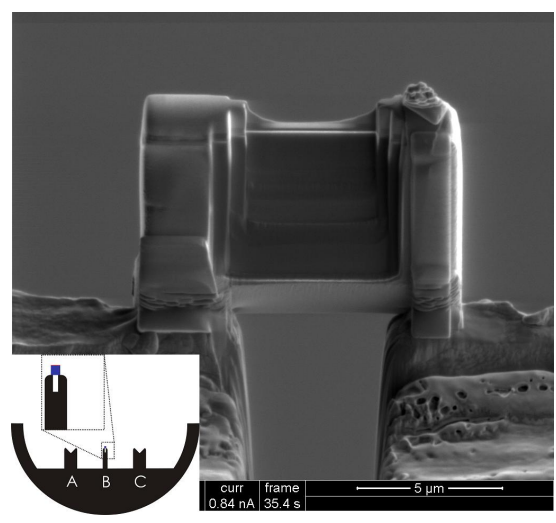

(a)

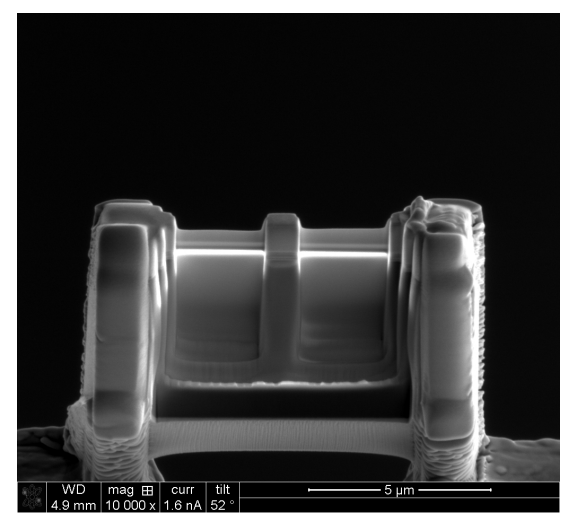

(b)

Abbildung 3.10.: a) Anbringung der Lamelle auf dem Träger: Der mittlere Steg wurde eingeschnitten und die Probe über diesem Schlitz positioniert. b) TEM-Lamelle mit stabilisierendem Steg in der Mitte. 
Statt eines TEM-Probenträger aus $\mathrm{Cu}$ wird Mo verwendet, weil dieser sputterresistenter ist und dadurch weniger Trägermaterial durch den breiten ArIonenstrahl abgetragen wird, das sich auf der TEM-Probe ablagern könnte.

Damit die TEM-Lamelle im PIPS im Zentrum der Ionenstrahlen liegt, wird die Lamelle auf dem mittleren Steg des TEM-Probenträgers montiert. Zuvor wird ein tiefer Schlitz in diesen mittleren Steg gesputtert (s. Abb. 3.10a).

Dieser Schlitz wird schon vor dem Aufbringen der Lamelle im FIB geschnitten und dient ebenfalls dazu, die Redeposition vom Trägermaterial beim Dünnprozess, sowohl im FIB als auch im PIPS zu minimieren.

Da sich das MgO beim Dünnen im FIB sichtbar auflädt und sich infolgedessen verbiegt, wird beim letzten Dünnschritt im FIB ein stabilisierender Steg in der Mitte stehen gelassen (s. Abb. 3.10b). Dieser teilt die Probe in zwei Bereiche ein, die im TEM eine deutlich verringerte Probenverbiegung aufweisen als übliche Lamellen mit einem ca. $5 \mu \mathrm{m}$ breiten, durchgängig dünnen Bereich.

Im Anschluss an die FIB-Präparation wird der Probenträger in das PIPS eingebaut. Dabei ist darauf zu achten, dass die Proben bei der Sektordünnung nur von vorn gedünnt werden, um kein Trägermaterial auf die Probe zu sputtern (,Single Beam“). Die Ionenquellen werden hier jeweils unter einem Winkel von $\pm 7^{\circ}$ senkrecht zur Probennormalen eingesetzt und die Probe mit $3 \mathrm{U} /$ Min rotiert. Die eingesetzten Beschleunigungsspannungen und Zeiten sind in Tabelle 3.3 aufgeführt. Je geringer die Beschleunigungsspannung ist, desto oberflächensensitiver ist der Materialabtrag, weshalb bei den letzten Dünn-

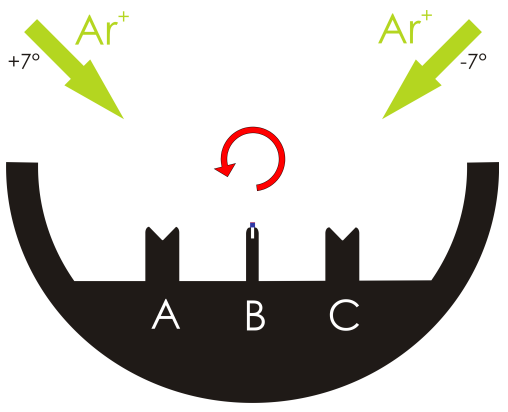

Abbildung 3.11.: Skizze des rotierenden Probenträgers im PIPS. schritten nur noch wenig Material abgetragen wird und hauptsächlich der Reduktion amorpher Deckschichten dient. Die Ätzwinkel sind bei $\pm 7^{\circ}$ belassen; es wird nur die Beschleunigungsspannung reduziert, da diese für die Dicke der amorphen Deckschichten der präparierten TEM-Lamellen von größeren Bedeutung ist [105].

Die Dicke der Probe kann im PIPS nicht kontrolliert werden. Deshalb wird die Dicke der hier untersuchten Lamellen immer wieder im TEM kontrolliert. Dabei werden Dickenverteilungskarten mittels EFTEM erstellt. Außerdem ist eine Position des zu untersuchenden Probenbereichs nur wenige $10 \mathrm{~nm}$ von der Keilspitze entfernt ein guter Hinweis auf die Dicke der Lamelle.

Für das Materialsystem $\mathrm{MgO} / \mathrm{CoFeB}$ werden die besten Ergebnisse mit der in Tabelle 3.3 skizzierten Sputterkaskade erzielt.

In Abbildung $3.12 \mathrm{a}$ und Abbildung $3.12 \mathrm{~b}$ sind zwei TEM-Abbildungen dar- 




(a)

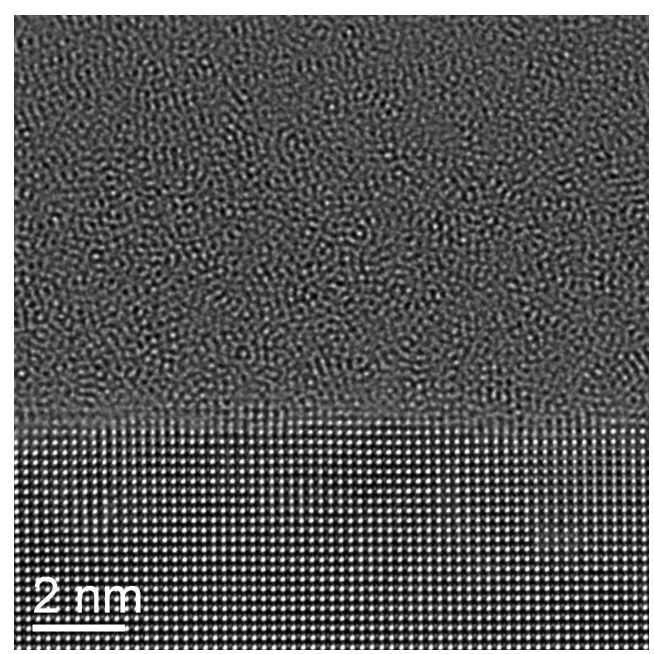

(c)

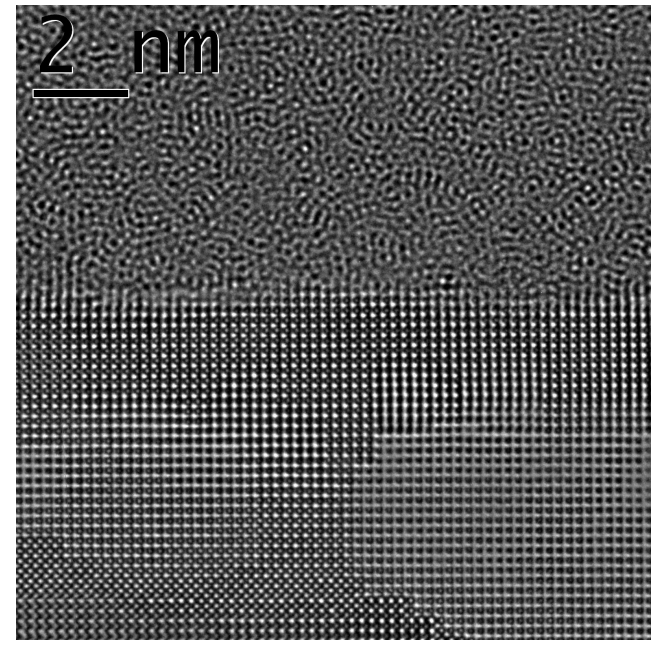

(b)

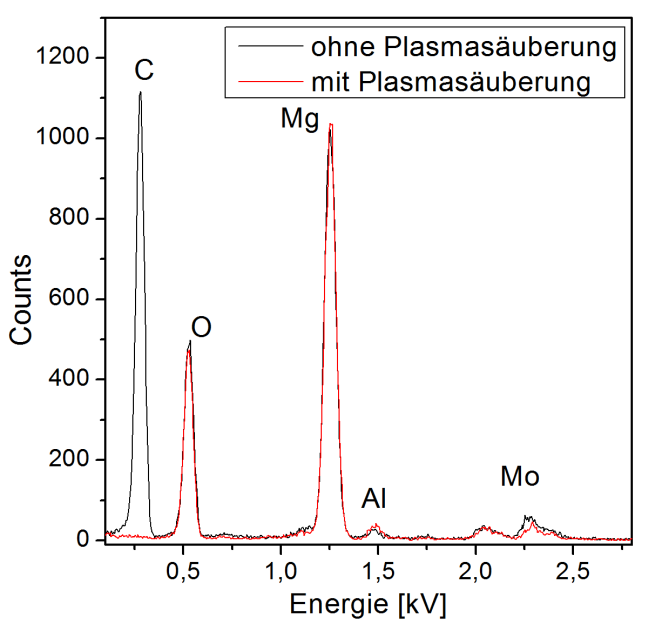

(d)

Abbildung 3.12.: a) Die HRTEM-Abbildung zeigen deutlich ein stufenförmiges und plattenweises Abplatzen des MgO beim Dünnprozess. b) HRTEM-Abbildung mit deutlich sichtbaren, unterschiedlich dicken Regionen im $M g O$, erkennbar an den unterschiedlichen kristallinen Mustern. c) HRTEM-Abbildung der MgO/CoFeB-Grenzfläche einer Probe, die im Vergleich zu b) eine deutlich geringere Dickenvariation aufweist. d) EDX-Messung einer Probe im Bereich des MgO-Substrats: mit vorheriger Plasmasäuberung (rot) und ohne vorheriger Plasmasäuberung (schwarz). In der Messung ohne vorheriger Säuberung ist ein deutliches Kohlenstoffsignal bei 0,277 kV messbar, das nach der Plasmasäuberung nicht mehr vorhanden ist. 


\begin{tabular}{|l|l|}
\hline $2,0 \mathrm{kV}$ & 2,5 Min. \\
\hline $1,0 \mathrm{kV}$ & 5 Min. \\
\hline $0,5 \mathrm{kV}$ & 15 Min. \\
\hline
\end{tabular}

Tabelle 3.3.: In der Tabelle dargestellt sind die hier verwendeten PIPS-Parameter. Besonders die Dauer des $2 k V$ Dünnschrittes ist abhängig von der Probendicke.

gestellt, bei denen es bei abweichenden Parametern zu deutlich sichtbaren Inhomogenitäten in der Probe kam. In Abbildung 3.12a sind die für $\mathrm{MgO}$ typischen rechtwinkligen Abplatzungen am Rand der Probe zu sehen. Abbildung $3.12 \mathrm{~b}$ weist ebenfalls diese rechtwinkligen Bereiche mit unterschiedlichem kristallinem Muster im $\mathrm{MgO}$ auf, welche durch Dickenunterschiede nach dem Abplatzen von MgO-Schichten hervorgerufen werden. Durch diese starken Dickenvariationen wird ein Vergleich der über einen weiten Bereich gemittelten experimentellen und simulierten Abbildungen unmöglich. Abbildung 3.12c zeigt im Vergleich dazu eine Probenstelle, bei der diese unterschiedlichen $\mathrm{Mu}-$ ster im Kristallinen nicht so stark ausgeprägt sind, weshalb man hier von einer gleichmäßig dünnen Probe ausgehen kann.

Direkt vor dem Einbau der Proben in das TEM wurde der FIB-Probenträger für 10 Minuten in einem Plasma Cleaner der Firma „Plasma Technology“ Typ „MiniFlecto“ bei 70 \% Leistung gesäubert. Durch diesen Prozess konnten amorphe Kohlenstoffdeckschichten, die die Probe im FIB kontaminieren und insbesondere EELS-Messungen behindern, zuverlässig entfernt werden (s. Abb. $3.12 \mathrm{~d}$. 
3. Experimentelle Methoden 


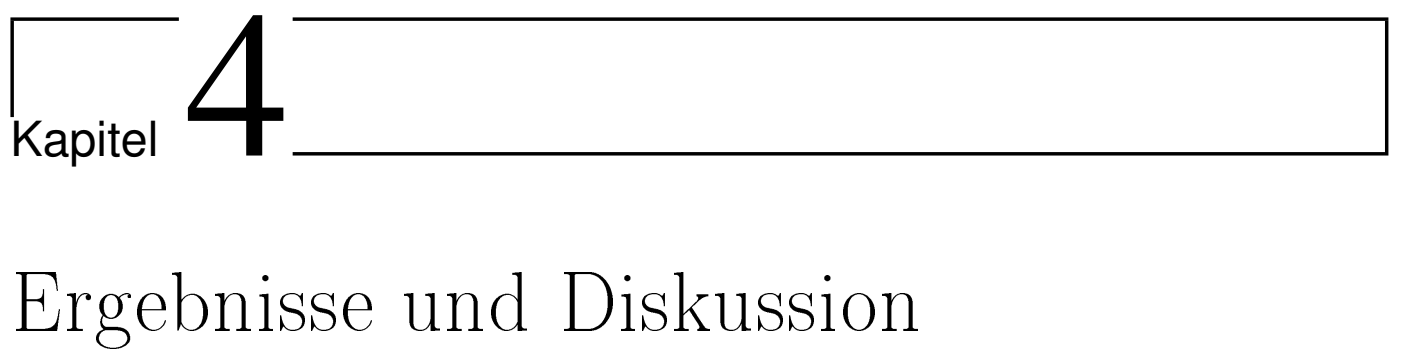

Die Untersuchungen in diesem Kapitel werden in drei Abschnitte eingeteilt: Der erste Abschnitt (Abschnitt 4.1) setzt sich mit der Struktur der Grenzfläche zwischen $\mathrm{c}-\mathrm{MgO}$ und $\mathrm{c}-\mathrm{CoFe}$ bzw. a-CoFeB auseinander. Anschließend behandelt der zweite Teil (Abschnitt 4.2) den Themenbereich der Bor-Diffusion und der Kristallisation an der Deckschicht anhand von Modellsystemen und realen MTJ. Zum Schluss werden im dritten Abschnitt (Abschnitt 4.3) Untersuchungen an überspannungszerstörten MTJ vorgestellt.

\subsection{Strukturelle Eigenschaften der $\mathrm{MgO} / \mathrm{CoFe}(\mathrm{B})-$ Grenzfläche}

In Abschnitt 2.2 wurde gezeigt, dass dem strukturellen Übergang an der Grenzfläche zwischen der isolierenden MgO-Tunnelbarriere und den ferromagnetischen Elektroden eine entscheidende Bedeutung zum Erzielen hoher TMRWerte in diesen Systemen zukommt. Heiliger und Gradhand et al. [11, 44] konnten berechnen, dass bereits wenige Monolagen kristallinen Eisens an der Grenzfläche ausreichen müssten, um hohe TMR zu erhalten. Diese Grenzfläche zwischen $\mathrm{MgO}$ und $\mathrm{CoFeB}$ soll in den folgenden Abschnitten untersucht werden. Dazu wird zuerst die kristallin/kristalline Grenzfläche (Abschnitt 4.1.1) zwischen $\mathrm{MgO}$ und $\mathrm{CoFe}$ untersucht, um daraus Erkenntnisse abzuleiten, welche für die Modellierung des Übergangsbereichs (s. Abschnitt 3.3.4) zwischen kristallinem $\mathrm{MgO}$ und amorphem CoFeB wichtig sind. In Abschnitt 4.1.2 wird diese Grenzfläche an nicht ausgelagerten Systemen untersucht. Der Fokus liegt hierbei in der Quantifizierung der Reichweite der durch das $\mathrm{MgO}$ induzierten kristallinen Ordnung ins CoFeB. 
Die TEM-Untersuchungen erfolgen in diesem Abschnitt an einem einfachen Modellsystem. Sie besteht aus einer auf ein MgO-Einkristall aufgebrachten CoFeB-Schicht. Da in realen MTJs die MgO-Tunnelbarriere texturiert aufwächst, ist so eine Kontrolle der Kristallorientierung bei der Präparation möglich. Eine genaue Beschreibung des Probenaufbaus und der Probenherstellung befindet sich im Anhang unter Abschnitt B.1 und Abschnitt B.2.

\subsubsection{Die c-MgO/c-CoFe-Grenzfläche}

Die kristallin/kristalline Grenzfläche zwischen $\mathrm{MgO}$ und $\mathrm{CoFe}$ wird untersucht, um hieraus Annahmen für die Konstruktion der dreidimensionalen Verteilungsfunktion ableiten zu können, welche für die Untersuchung der kristallin/amorphen Grenzfläche gebraucht werden.

Dazu soll im Folgenden beantwortet werden, welche Bindungspartner an der Grenzfläche vorhanden sind und wie sich die Bindungsrichtungen und -abstände zwischen diesen verhalten.

Um überhaupt eine Aussage über die Bindungspartner an der Grenzfläche treffen zu können, muss die Probe entlang der MgO[110]Richtung orientiert werden. In dieser Orientierung befinden sich die Sauerstoff- und die Mag-

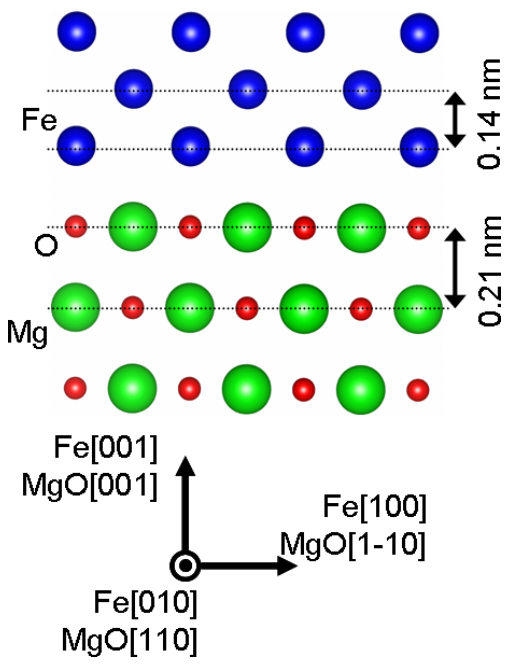

Abbildung 4.1.: Pseudomorph gewachsenes $\mathrm{Fe}(001)$ auf $\mathrm{MgO}(001)$. nesiumatome in separaten Atomsäulen entlang der Strahlrichtung des TEMs (s. Abb. 4.1).

Die Untersuchung der Grenzfläche wurde an der Phase der Objektwelle durchgeführt, welche man aus der Objektwellenrekonstruktion erhält (s. Abschnitt 3.1.3). Die dafür erforderliche Defokusserie aus 20 Abbildungen wurde bei geringer negativ sphärischer Aberration von $C_{s}=-7 \mu \mathrm{m}$ aufgenommen. Der Startdefokus der Abbildungsserie lag bei $\Delta f=20 \mathrm{~nm}$ bei einer Defokusschrittweite von $\delta \Delta f=2,0 \mathrm{~nm}$. Die nach der Korrektur des Mikroskops noch vorhandenen Linsenfehler wurden mittels eines Zemlin-Tableaus am Mikroskop bestimmt und die in Tabelle 4.1 angegebenen Werte anschließend genutzt, um die rekonstruierte Objektwelle numerisch zu korrigieren.

Abbildung 4.2 zeigt die rekonstruierte Phase der Objektwelle, in der das 


\begin{tabular}{|c|c|c|}
\hline Linsenfehler & Betrag & Vertrauensintervall \\
\hline zweizähliger Astigmatismus & $1,4 \mathrm{~nm}$ & $1,5 \mathrm{mn}$ \\
\hline axiale Koma & $3 \mathrm{~nm}$ & $19,5 \mathrm{~nm}$ \\
\hline dreizähliger Astigmatismus & $46 \mathrm{~nm}$ & $33 \mathrm{~nm}$ \\
\hline
\end{tabular}

Tabelle 4.1.: Aus dem Zemlin-Tableau bestimmte Linsenfehler, die für die numerische Korrektur der Objektwelle genutzt wurden.



Abbildung 4.2.: Abbildung der rekonstruierten Phase: MgO[110] unten, CoFe[010] oben. Das eingebettete Profil aus dem rot eingezeichneten Bereich zeigt den Kontrastunterschied zwischen $\mathrm{Mg}$ (heller) und O (dunkler); Die eingebetteten Abbildungen in der oberen Hälfte zeigen Ausschnitte der fouriergefilterten Abbildung der Phasen. Diese stammen aus den Regionen, die mit roten Kreisen versehenen und an denen eine Versetzung im CoFe zu finden sind. 
CoFe in einer bcc-Kristallstruktur kristallisiert ist und $45^{\circ}$ verdreht auf dem $\mathrm{MgO}$ aufgewachsen ist. Da der leichtere Sauerstoff einen geringeren Phasenschub hervorruft als das schwerere Magnesium, weist $\mathrm{O}$ einen geringeren Kontrast auf als Mg. Dies ist gut in dem eingezeichneten Profil in Abbildung $4.2 \mathrm{zu}$ erkennen, das entlang der $\mathrm{MgO}[1 \overline{1} 0]$-Richtung im rot eingezeichneten Bereich erstellt wurde. Aufgrund der Gitterfehlpassung von 4,3\% kommt es aufgrund von Relaxationen an der Grenzfläche im CoFe zu Versetzungen entlang der [100]-Richtung. Diese sind deutlich in der fouriergefilterten Abbildung in 4.2 zu sehen und rot eingekreist. In dieser Abbildung sind auf $16 \mathrm{~nm}$ drei Versetzungen sichtbar. Wegen der Gitterfehlpassung ist etwa alle 7,3 $\mathrm{nm}$ eine Versetzung zu erwarten. An der Grenzfläche abseits der Versetzungen ist aufgrund der Kontrastunterschiede zwischen $\mathrm{Mg}$ und $\mathrm{O}$ zu erkennen, dass das $\mathrm{Co} / \mathrm{Fe}$ senkrecht zur Grenzfläche über den dunkleren O-Gitterplätzen steht. Aufgrund der durch Fehlpassung hervorgerufenen Versetzungen und die dazwischenliegenden elastisch verzerrten Bereiche, handelt es sich bei der $\mathrm{MgO} / \mathrm{CoFe}-$ Grenzfläche um eine semikohärente Grenzfläche. Darüber hinaus weist die Grenzfläche auch einige Stufen bzw. Inseln auf. 


\subsubsection{Bestimmung des Gitterabstands senkrecht zur Grenzfläche}

Im Folgenden sollen der Gitterabstand senkrecht zur Grenzfläche zwischen dem letzten Sauerstoffatom des $\mathrm{MgO}$ und des ersten $\mathrm{Fe} / \mathrm{Co}-$ Atoms des CoFe bestimmt werden. Hierfür werden in der rekonstruierten Phase (s. Abb. 4.2) Intensitätsprofile in einem durch Versetzungen nicht beeinflussten Bereich gebildet. Diese Profile werden entlang der senkrechten Pfeile in Abbildung 4.3 gebildet und sind an der Grenzfläche sauerstoffterminiert. Aus diesen Profilen (s. Abb. 4.4a) werden die mittleren Intensitätsmaxima bestimmt und deren Abstände in Abbildung $4.4 \mathrm{~b}$ aufgetragen. Dabei ist jeweils der Abstand zum vorherigen Maximum dargestellt. Im Bereich des $\mathrm{MgO}$ zeigt sich bei dieser Auswertung ein mittlerer Atomabstand im $\mathrm{MgO}$ von $0,212 \pm 0,01 \mathrm{~nm}$ senkrecht zur Grenzfläche, welcher innerhalb des Fehlers zum theoretisch erwarteten Wert der halben Gitterkonstante im $\mathrm{MgO}$ von $0,211 \mathrm{~nm}$ liegt. Im CoFe ergibt sich ein mittlerer Atomabstand von 0,290 $\pm 0,01 \mathrm{~nm}$, der ebenfalls unter Berücksichtigung des Fehlers mit dem zu erwartenden Wert von etwa $0,285 \mathrm{~nm}$ übereinstimmt. Die Bindungslänge der ersten Fe-Lage an das letzte Sauerstoffatom ist hier mit 0,184 0,02 nm bestimmt. Dieser Wert ist im Vergleich mit den in Abschnitt 3.3.4 diskutierten Werten recht klein. So weisen z.B. die von Burton et. al vorgenommenen Berechnungen an der MgO-CoFeB-Grenzfläche 24] einen etwa $20 \%$ größeren Abstand auf. 
a)

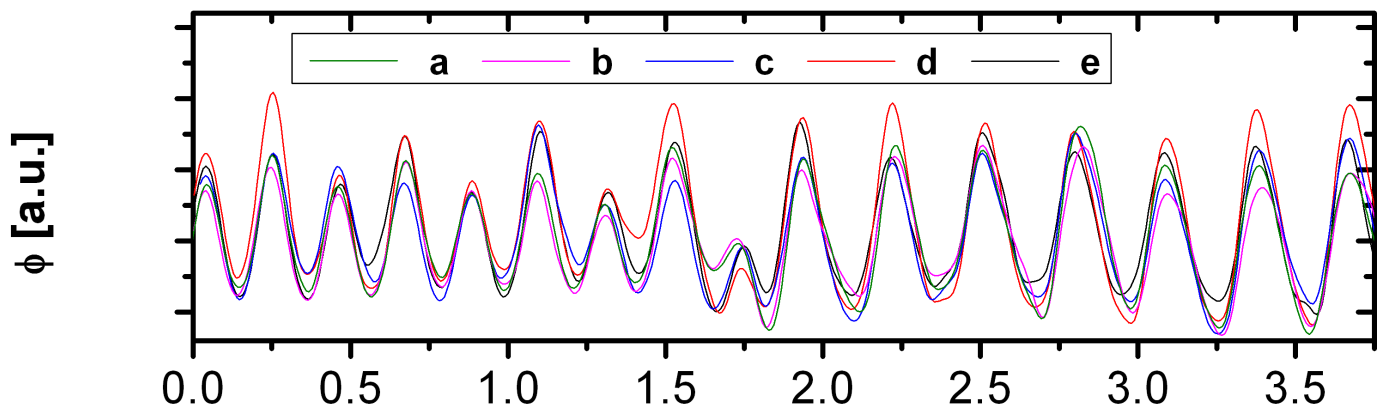

b) $\mathbf{x}[\mathrm{nm}]$

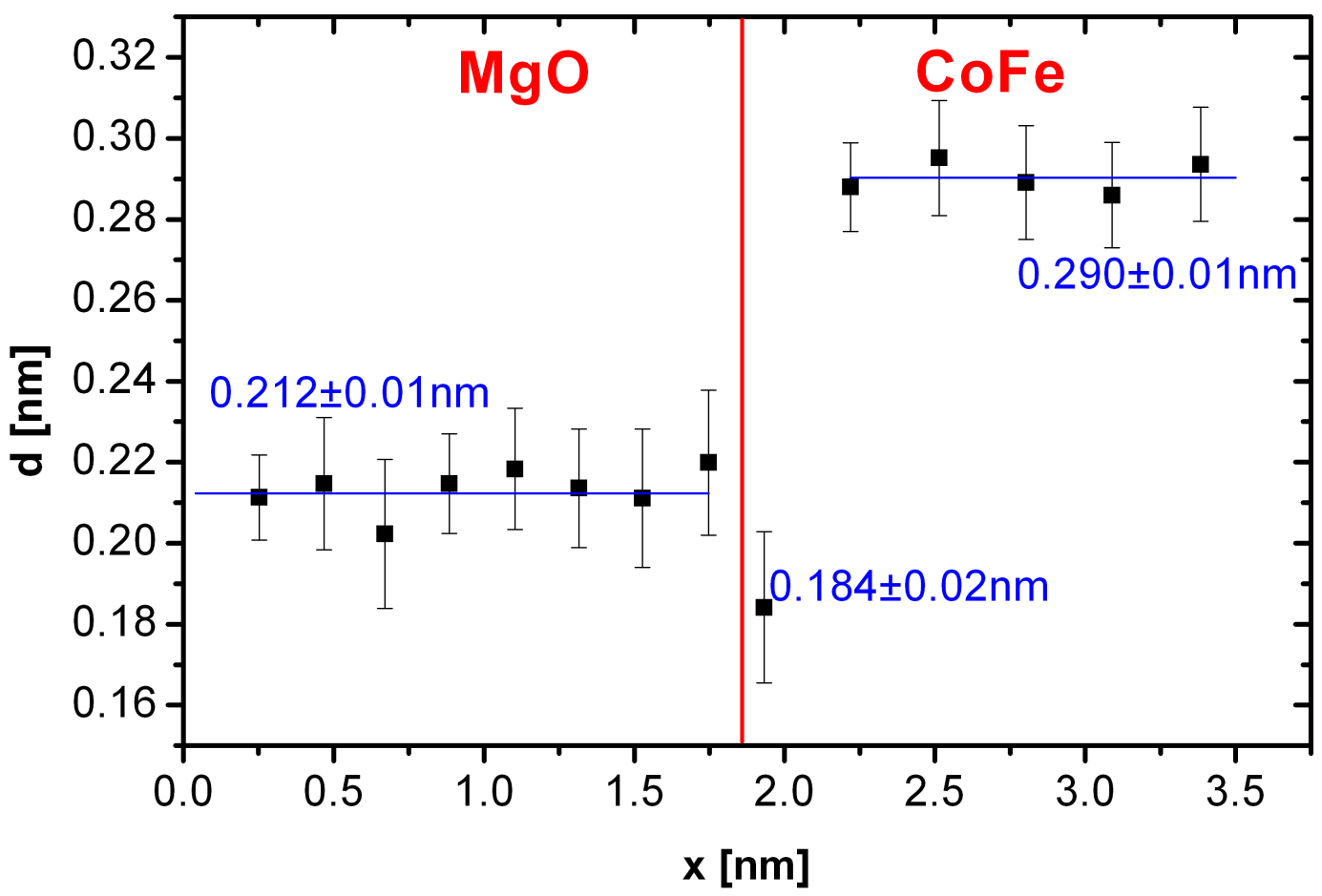

Abbildung 4.4.: a) Darstellung der sauerstoffterminierten Intensitätsprofile über die Grenzfläche. b) Interplanarer Gitterabstand bestimmt aus der Position der Intensitätsmaxima von 4.4 a gemessen senkrecht zum Übergang vom $\mathrm{MgO}$ ins CoFe aus der rekonstruierten Phase. 


\subsubsection{Sauerstoffversatz an der Grenzfläche}

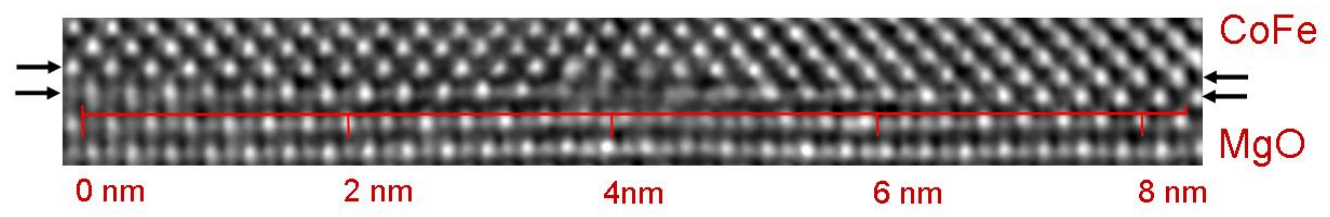

Abbildung 4.5.: Rekonstruierte Phase mit eingezeichneten Bereichen (Pfeile), in denen der Intensitätsverlauf 4.7 a parallel zu Grenzfläche gemessen wurde.

Um das Bindungsverhältnis an der Grenzfläche genauer bestimmen $\mathrm{zu}$ können, wird im Folgenden der Sauerstoffversatz parallel zur Grenzfläche und in der Nähe einer Versetzung untersucht. Insofern eine Bindung zwischen $\mathrm{O}$ und $\mathrm{Fe}$ besteht, sollte in der Nähe von Versetzungen das $\mathrm{O}$ durch dessen Bindung mit Fe aus seiner Lage in der Mitte zwischen zwei Mg-Atomen verschoben werden. Um diese Verschiebung messen zu können, werden in der Abbildung der rekonstruierten Phase zwei Intensitätsprofile parallel zur Grenzfläche gebildet, die sich jeweils entlang der letzten MgO-Lage und der ersten CoFe-Lage parallel zur Grenzfläche befinden (s. Abb. 4.5).

Wie schon in Abbildung 4.2 erkennbar, befinden sich entlang der Grenzfläche mehrere Versetzungen. Dort ist ersichtlich, dass diese Versetzung vor allem im CoFe sitzt, da das $\mathrm{MgO}$ darunter nahezu unbeeinflusst ist. In der hier untersuchten Abbildung 4.5 befindet sich die Versetzung bei etwa $x=4,5 \mathrm{~nm}$. Die Profile sind in Abbildung 4.7a dargestellt. In der letzten MgO-Lage (hier rot dargestellt) ist der Kontrastunterschied zwischen dem dunkleren Sauerstoff und den helleren Magnesiumatomen sichtbar. In der erste CoFe-Lage, hier in Blau dargestellt, sind keine regelmäßigen Kontrastunterschiede sichtbar; lediglich bei $x=1,5-2,0 \mathrm{~nm}$ ist ein kleiner Peak zwischen zwei Maxima vorhanden. Ursache hierfür könnte eine 
a)
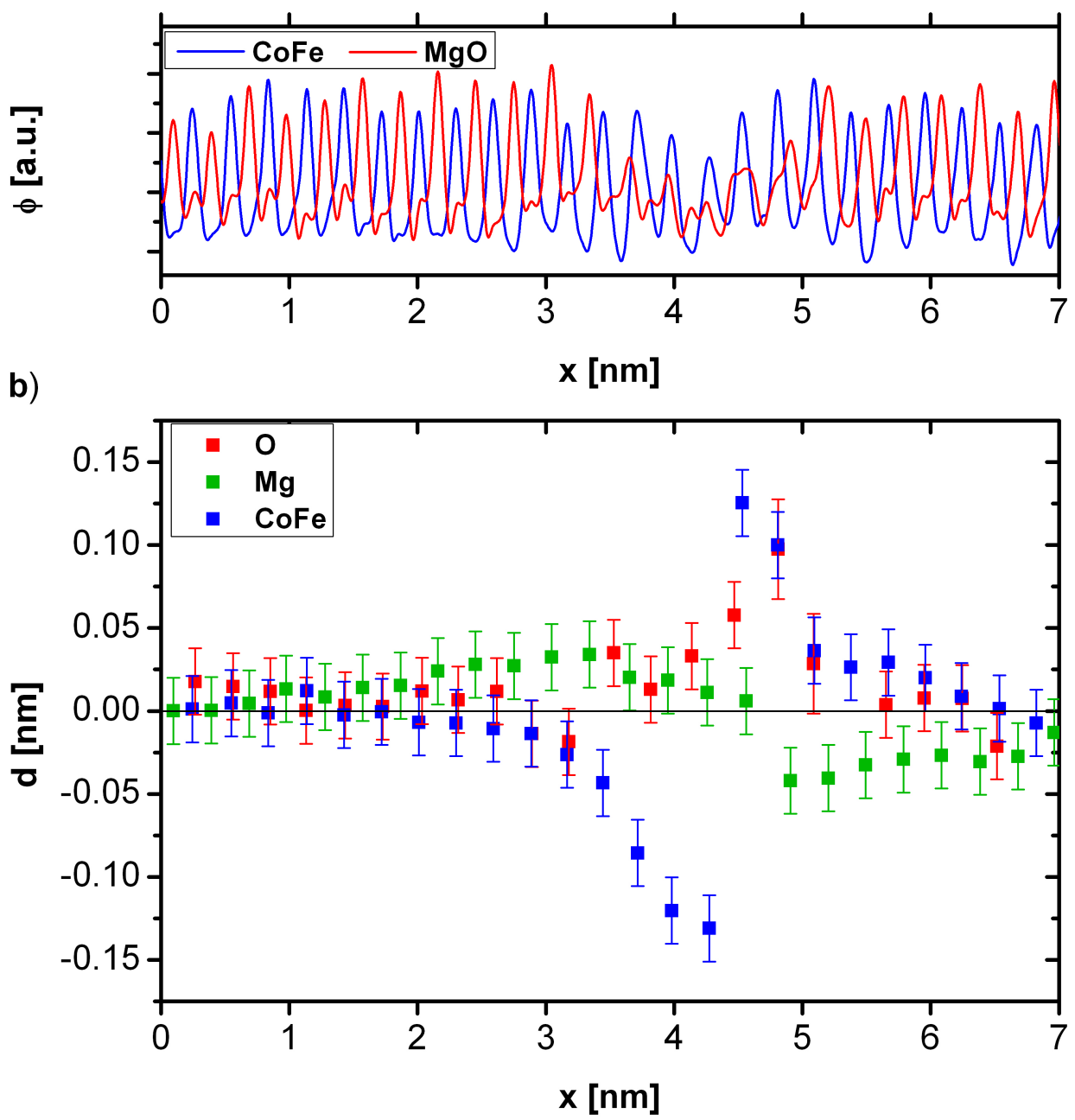

\begin{abstract}
Abbildung 4.7.: a) Intensitätsverlauf parallel zur Grenzfläche der letzten MgO-Lage (rot) und der ersten CoFe-Lage (blau). Gut sichtbar der unverzerrte Kristallbereich links und das durch eine Stufenversetzung im Bereich um $x=4,5 \mathrm{~nm}$ hervorgerufene Versetzungsfeld im CoFe. b) Verschiebung der Atome aus der in 4.6 skizzierten Position. Positive Werte zeigen eine Verschiebung nach rechts an. Gut zu erkennen ist die Versetzung bei $x=4,5 \mathrm{~nm}$ in der Verschiebung der CoFe-Atome (blau). Ebenfalls deutlich ist, dass die Mg-Atome den CoFe-Atomen in der Nähe der Versetzung nur leicht folgen. Die Sauerstoffatome werden stärker durch das CoFe beeinflusst, z.B. bei $x=3,2 \mathrm{~nm}$ oder im Bereich von $x=4,5 \mathrm{~nm}$ bis $x=6,2 \mathrm{~nm}$.
\end{abstract}


Stufe an der Grenzfläche oder eine geringe Oxidation der Grenzfläche sein, bei der ein Sauerstoffatom zwischen zwei Fe-Atomen sitzt. Aus den beiden Profilen werden die Verschiebungen der Atomlagen, wie in Abbildung 4.6 skizziert, gebildet (s. Abb. 4.7b). Dabei wird in Grün die Auslenkung des Mg-Atoms aus seiner mittleren Position aufgetragen, in Rot die Auslenkung des Sauerstoffs aus der Mitte seiner beiden benachbarten Mg-Atome sowie in Blau der Abstand der CoFe-Atome von der nächsten, mittleren Position zwischen zwei Mg-Atomen. Ein positiver Versatz eines Atoms bedeutet eine Auslenkung im Realbild nach rechts. In dieser Auftragung wird die Stufenversetzung im CoFe anhand des Sprungs der Auslenkung gut sichtbar. Der Versetzungskern befindet sich etwa bei $x=4,5 \mathrm{~nm}$. Die Mg-Atome scheinen von der Versetzung im CoFe nahezu unbeeinflusst; sie sind kaum aus ihrer mittleren Position ausgelenkt. Die Sauerstoffatome sind jedoch stärker an die $\mathrm{Co} / \mathrm{Fe}-$ Atome gebunden; sie folgen vorwiegend den $\mathrm{Co} / \mathrm{Fe}$-Atomen im Verzerrungsfeld um die Versetzung herum, etwa bei $\mathrm{x}=3,2 \mathrm{~nm}$ oder im Bereich von $\mathrm{x}=4,5 \mathrm{~nm}$ bis $\mathrm{x}=6,2 \mathrm{~nm}$. In der Nähe des Versetzungskerns befinden sich die O-Atome jedoch in einem Spannungsfeld aus den Einflüssen mehrerer CoFe- und $\mathrm{Mg}$-Atome und folgen dem CoFe somit nicht mehr eindeutig. In einem Abstand von ca. 1,5 nm vom Versetzungskern ist das Kristallgitter, im Rahmen der Messgenauigkeit, ungestört.

Da es sich beim CoFe um einen ungeordneten Kristall handelt, ist eine Unterscheidung zwischen Fe und Co nicht möglich. Somit kann hier auch keine Aussage bezüglich des Bindungspartners von Sauerstoff im CoFe getroffen werden. Ebenso wenig lassen die Dissoziationsenergien (s. Tab. 4.2) von Fe-O und Co-O (aus [106]) klare Aussaugen hierzu zu.

\begin{tabular}{|c|c|}
\hline Bindung & $\Delta H f[\mathrm{~kJ} / \mathrm{mol}]$ \\
\hline $\mathrm{Mg}-\mathrm{O}$ & $394(35)$ \\
\hline $\mathrm{Fe}-\mathrm{O}$ & $409(13)$ \\
\hline $\mathrm{Co}-\mathrm{O}$ & $368(21)$ \\
\hline
\end{tabular}

Tabelle 4.2.: Dissoziationsenergien von $\mathrm{Mg}-\mathrm{o}$, Fe-O und Co-O. Aus [106].

\subsubsection{Zusammenfassende Diskussion der Ergebnisse zur Untersuchung an der c-MgO/c-CoFe-Grenzfläche}

Zusammenfassend kann man die in Abschnitt 3.3.4 genutzten Annahmen bestätigen. Das CoFe kristallisiert beim Auslagern in einer bcc-Kristallstruktur, welches das $\mathrm{MgO}$ als Kristallisationsgrundlage nimmt. Aufgrund der Gitterfehlpassung kommt es in dem vollständig kristallisierten Zustand bei diesen 
Schichtdicken zu Versetzungen im CoFe. Diese Versetzungen wurden so auch in Fe-MgO-Systemen im Fe gefunden [107]. Durch die Untersuchung mittels Objektwellenrekonstuktion ist eine Unterscheidung der mit Sauerstoff und Magnesium besetzten Gitterplätze in der rekonstruierten Phase möglich.

Aufgrund des Verhaltens der Sauerstoffatome in der Nähe von Versetzungen im CoFe ist von einer Bindung zwischen Fe/Co und Sauerstoff auszugehen. Außerdem wurde beobachtet, dass das erste $\mathrm{Co} / \mathrm{Fe}$-Atom senkrecht zur Grenzfläche über dem letzten Sauerstoff steht. Der Abstand der Sauerstoff- und Fe/Co-Gitterplätze senkrecht zur Grenzfläche mit 0, $184 \pm 0.02 \mathrm{~nm}$ passt mit etwa $4 \%$ Abweichung zu den Fe-O-Abständen $d_{F e-O}=0,193 \mathrm{~nm}$ im Eisen(III)oxid [108]. Berechnungen von Burton et. al [24] für den Abstand zwischen $\mathrm{Fe}-\mathrm{O}$ in $\mathrm{MgO}-\mathrm{CoFeB}-\mathrm{Systemen}$ von $d_{\mathrm{Fe}-\mathrm{O}}=0,223 \mathrm{~nm}$ weisen jedoch einen etwa $20 \%$ größeren Abstand auf. Ebenso verhält es sich mit dem von Wang et al. [107] experimentell gemessenen Abstand zwischen Fe und $\mathrm{O}$ in MgO-FeSystemen von $d_{F e-O}=0,220 \mathrm{~nm}$. 


\subsubsection{Struktureller Übergang der c-MgO/a-CoFeB-Grenzfläche}

Heiliger und Gradhand et al. [11, 44] konnten in Berechnungen zeigen, dass im Idealfall bereits eine Monolage kristallinen bcc-Eisens an der Grenzfläche zur MgO-Tunnelbarriere ausreichen sollte, um einen TMR von $570 \%$ zu erzielen. Experimentell konnten Djayaprawira et al. [10] an Tunnelelementen, die eine weitgehend amorphe CoFeB-Elektrode aufwiesen, hohe TMR von $230 \%$ messen.

In diesem Abschnitt soll anhand der in Abschnitt 3.3 vorgestellten Methode der strukturelle Übergang an der Grenzfläche von kristallinem MgO zu amorphem $\mathrm{CoFeB}$ untersucht werden. In funktionalen MTJs wird das $\mathrm{MgO}$ auf amorphes $\mathrm{CoFeB}$ aufgewachsen, weshalb es in keiner epitaktischen Beziehung zum Substrat steht und darüber hinaus texturiert aufwächst [2]. Da für die in Abschnitt 3.3 vorgestellte Methode die Kenntnis der MgO-Kristallorientierung und die Einkristallinität fundamentale Voraussetzungen sind, wird die Untersuchung, wie im vorangegangenen Abschnitt, an einem Modellsystem durchgeführt. Das Modellsystem ist im Anhang unter Abschnitt B.2 genauer beschrieben und besteht im Wesentlichen aus einer auf einem einkristallinen MgOSubstrat aufgebrachten amorphen CoFeB-Schicht.

Durch die an diesem Modellsystem gewonnenen Erkenntnissen über die Struktur an der Grenzfläche wird im Anschluss die Ausdehnung des vom MgO modifizierten Übergangsbereiches bestimmt. Durch die Untersuchung von nicht ausgelagerten a-CoFeB-Schichten ist es dadurch möglich, die minimale Ausdehnung dieses Übergangsbereiches zu ermitteln und diese mit dem von Heiliger et al. [11] berechneten Modell in Beziehung zu setzen.

Die hochauflösenden Transmissionselektronenmikroskopischen Abbildungen werden mit dem abbildungskorrigierten FEI TITAN ETEM entlang der [100]Richtung des $\mathrm{MgO}$ durchgeführt (s. Abb. 3.7.). Als Mikroskop-Parameter in die Simulation eingeflossen sind die Beschleunigungsspannung von $300 \mathrm{kV}$, eine sphärische Aberration von $C_{s}=0 \mu \mathrm{m}$, die Defokusstreuung von $\Delta=2,9 \mathrm{~nm}$ und der Semikonvergenzwinkel der Beleuchtung von $\alpha=0,1 \mathrm{mrad}$.

Entsprechend des in Abschnitt 3.3.1 vorgestellten Verfahrens wird an dieser Probe zunächst eine experimentelle Defokusserie aus 20 Abbildungen erstellt und anschließend die Proben-Drift korrigiert. Die maximale Proben-Drift zwischen zwei Abbildungen liegt bei 0,08 nm mit einer Gesamtdrift von 0,67 nm, die im Wesentlichen entlang der Grenzfläche auftritt (s. Abb. 3.6. 
Grenzflächenposition:

4321

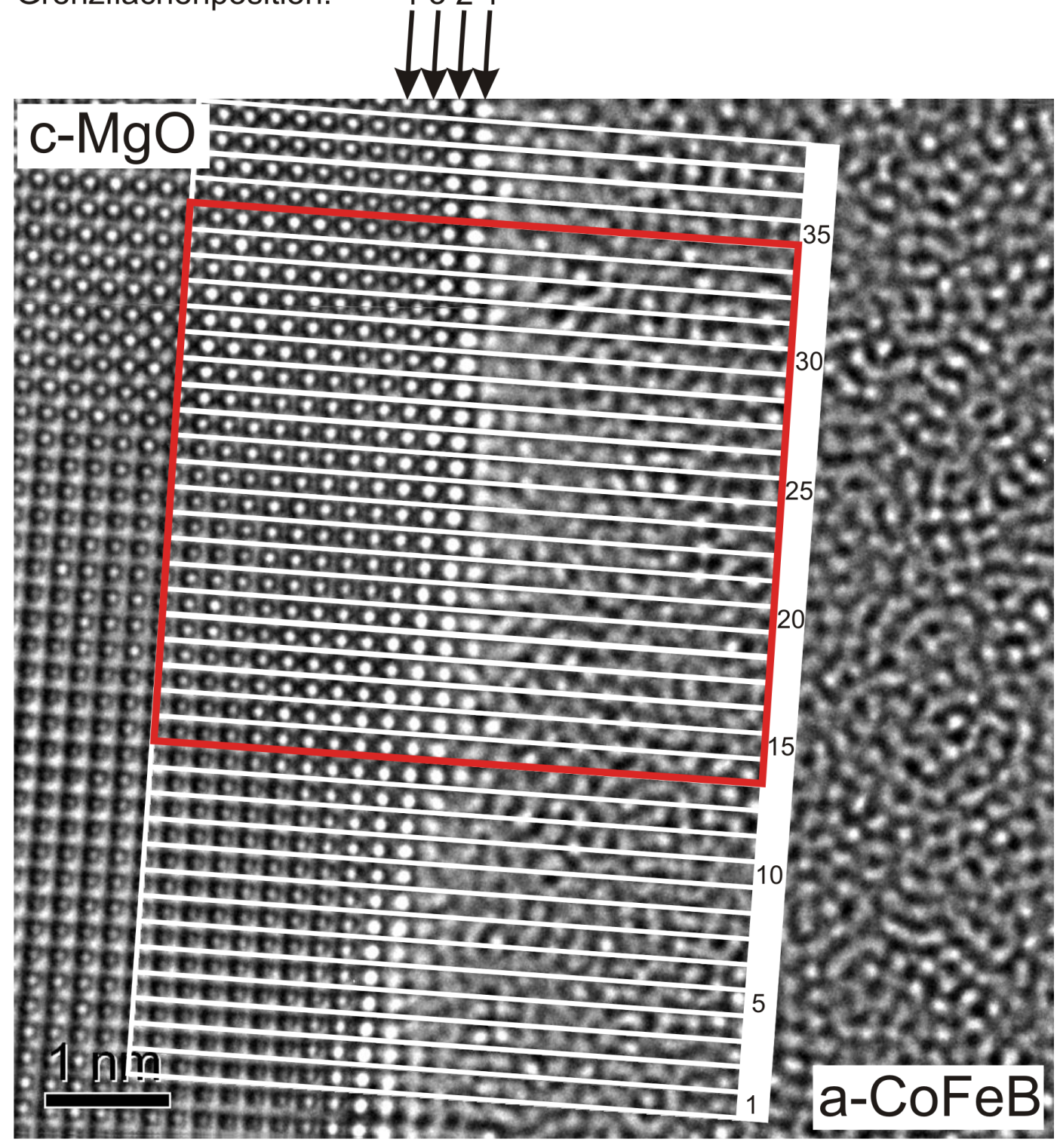

Abbildung 4.8.: HRTEM-Abbildung der c-MgO/a-CoFeB Grenzfläche bei einem Defokus von $\Delta f=1 \mathrm{~nm}$ mit Einteilung in Streifen senkrecht zur Grenzfläche. Mittelung über 20 Streifen ab 14. Streifen. 
Die ausgerichteten Abbildungen werden in Streifen parallel zur Grenzfläche eingeteilt und anschließend gemittelt. Für eine Beschreibung der Ordnung an der Grenzfläche mittels einer auf statistischen Beschreibungen beruhenden zweidimensionalen Verteilungsfunktion ist es per definitionem erforderlich, über einen möglichst großen Bereich entlang der Grenzfläche zu mitteln. Jedoch wird dieser Mittelungsbereich durch sichtbare, laterale, strukturelle Inhomogenitäten entlang der Grenzfläche eingeschränkt. Diese kleinen Stufen oder Inseln an der Grenzfläche konnten trotz Aufbringen einer $6 \mathrm{~nm}$ dicken MgO-Schicht auf das Substrat nicht weiter reduziert werden.

Trotz diese Unebenheiten müssen in den Abbildungen möglichst große Bereiche gefunden werden, über die gemittelt werden kann. Abbildung 4.8 zeigt den untersuchten Probenbereich bei einem Defokus von $\Delta f=1 \mathrm{~nm}$. Entlang der Grenzfläche ist die Abbildung in 38 Streifen senkrecht zur Grenzfläche eingeteilt. Aufgrund der deutlich sichtbaren, strukturellen Inhomogenitäten an der Grenzfläche wird für die folgenden Untersuchungen ein Mittelungsbereich von 20 Streifen (rot gekennzeichnet) ausgewählt, der entlang der Grenzfläche frei von sichtbaren Veränderungen ist.

Die notwendige Größe des Mittelungsbereiches wurden für das Modellsystem a-Ge/c-Si von Thiel [21] untersucht. Dort wurde die maximale relative Intensitätsvariation für verschiedene Ausdehnungen des Mittelungsbereiches bestimmt. Es zeigte sich, dass eine Anzahl von $\approx 30$ Streifen notwendig ist, um eine nicht-signifikante Intensitätsvariation von unter $\leq 5 \%$ zu erreichen. Nach der dortigen Untersuchung würden die hier vorhandenen 20 Streifen noch eine maximale Intensitätsvariation von $<10 \%$ aufweisen.

Intensitätsvariationen in den zu mittelnden Streifen führen zu Intensitätsvariationen in den gemittelten Grenzflächenabbildungen und somit zu Unsicherheiten in der zweidimensionalen Verteilungsfunktion $\rho(x, y)$. Ursächlich für diese Intensitätsvariationen sind:

- systematische Fehler: hervorgerufen durch Probenveränderungen während der Aufnahme der Defokusserie.

- statistische Fehler: durch „Schrotrauschen“ der CCD-Kamera.

- strukturelle Fehler: verursacht durch strukturelle Unterschiede entlang der Grenzfläche oder durch lateral unterschiedliche Reichweiten des kristallinen Kontrastes in einigen Bildbereichen.

Diese Fehler werden in der Regel durch eine große Anzahl von Messungen bestimmt, die hier aufgrund der Grenzflächenunebenheiten nicht möglich sind. Die Bootstrap-Methode bietet eine Möglichkeit den Fehler zu quantifizieren 
[109, 110]. Dabei werden mittels Monte-Carlo-Simulation synthetische Datensätze erzeugt. Diese bestehen aus einer festen Anzahl von Streifen, die willkürlich aus den vorhandenen, experimentell bestimmten Streifen zusammengestellt werden. Für diese Abschätzung sind 25-200 synthetische Datensätze notwendig. Für das Materialsystem a-Ge/c-Si ergab eine solche Abschätzung mittels Monte-Carlo-Simulation bei Thiel [21], dass sich zwar signifikante Unterschiede in der zweidimensionalen Verteilungsfunktion $\rho(x, y)$ für die von der Grenzfläche entfernteren Lagen ergeben, diese aber keinen Einfluss auf die Ausdehnung des Übergangsbereiches aufweisen. Für das System a-Si/c-Si war bei Thiel keine derartige Abschätzung möglich, da in diesem System, ähnlich dem hier untersuchten, deutliche strukturelle Inhomogenitäten entlang der Grenzfläche auftraten.

Darüber hinaus weist die APP-Näherung selbst einen systematischen Fehler auf, der von Borgardt et al. in Testrechnungen auf $\leq 5 \%$ [15] abgeschätzt werden konnte.

Aufgrund von Probenveränderungen entstandene, systematische Fehler, insbesondere solche, die durch lokale Kristallisation des CoFeB durch den Elektronenstrahl verursacht wurden, werden soweit wie möglich reduziert. Dies erfolgt durch eine Minimierung der Elektronendosis durch den Einsatz des Strahlblockers und die Reduktion des Strahlstroms mit Hilfe des Monochromators vor Aufnahme der Defokusserie.

In Abbildung 4.9 sind die aus 20 Streifen gemittelten experimentellen Abbildungen zusammengefasst dargestellt. Um das Kristallmuster besser erkennen zu können, ist dieser Streifen fünfmal untereinander abgebildet. Auf der linken Seite befindet sich das kristalline $\mathrm{MgO}$, auf der rechten das amorphe CoFeB. Die auftretenden Kontrastunterschiede im Amorphen, weit vom MgO entfernt, sind auf Mittelungsartefakte aufgrund der geringen Streifenanzahl zurückzuführen. Durch die sich wiederholende Darstellung erhält man den Eindruck von periodischen Strukturen im Amorphen parallel zur Grenzfläche.

Anhand der in Abschnitt 3.3.1 vorgestellten Verfahren wird die Probendicke, die Defokusschrittweite und der Startdefokus ermittelt, die in Tabelle $4.3 \mathrm{zu}-$ sammengefasst sind.

Anhand dieser Parameter werden anschließend die simulierten Grenzflächenabbildungen mit Hilfe der APP-Näherung simuliert. Die für die Simulation genutzte Superzelle hat ein Kantenlänge von $x=20 \cdot d_{M g O}=8,4220 \mathrm{~nm}$, $y=d_{M g O}=0,4211 \mathrm{~nm}$ und $z=d_{M g O}=0,4211 \mathrm{~nm}$, wobei sich die letzte Lage MgO bei $x=4,211 \mathrm{~nm}$ befindet.

Der quantitative Vergleich der simulierten und experimentellen Grenzflächenabbildungen mittels Berechnung des R-Faktors wurde im Übergangsbe- 


\begin{tabular}{|c|c|c|}
\hline Probendicke t & Startdefokus $\Delta f$ & Defokusschrittweite $\delta \Delta f$ \\
\hline $10,9 \mathrm{~nm}$ & $28,0 \mathrm{~nm}$ & $-3,0 \mathrm{~nm}$ \\
\hline
\end{tabular}

Tabelle 4.3.: Resultat der Probendickenbestimmung, des Startdefokus der Defokusserie und der Defokusschrittweite durch Vergleich der kristallperiodischen Kontraste von $\mathrm{MgO}$ aus gemittelten, experimentellen Abbildungen und simulierten Abbildungen der Defokusserie.

reich zwischen $3,6 \mathrm{~nm} \leq x \leq 6,0 \mathrm{~nm}$ der Superzelle durchgeführt. Dies führt dazu, dass nur Grenzflächeneffekte bewertet werden und mögliche Variationen, z.B. Dickenvariationen im kristallinem $\mathrm{MgO}$ bis $0,6 \mathrm{~nm}$ vor der letzten MgO-Lage nicht bewertet werden. Als Anpassungsalgorithmus für die iterative Parameteranpassung wurde, wie bei Thiel [21], die Simplex-Methode von Nelder und Mead verwendet [111, 112].

\subsubsection{Iterative Anpassung des strukturellen Übergangs an der Grenzfläche}

Die Position der Grenzfläche in den experimentellen Abbildungen ist im voraus nicht bekannt, weshalb für mehrere Grenzflächenpositionen eine iterative Bildanpassung durchgeführt wird. Diese möglichen Grenzflächenpositionen sind ebenfalls in Abbildung 4.8 eingezeichnet.

Um realistische Parameter für die Anpassung der zweidimensionalen Verteilungsfunktion zu bekommen, werden die Parameter in ihrem Anpassungsbereich eingeschränkt. So werden für alle Parameter positive Werte erwartet. Der Abstand $d_{a 1}$, der den Abstand der ersten CoFe-Lage vom O repräsentiert, wird auf den Bereich $0,1 \leq d_{a 1} \leq 0,3 \mathrm{~nm}$ beschränkt. Ebenfalls wird der Abstand $d_{a 2}$, also der Abstand zwischen den Co/Fe-Atomen auf den Bereich 0,08 nm $\leq d_{a 2} \leq 0,2 \mathrm{~nm}$ beschränkt, da kristallines CoFe einen halben Gitterabstand von $0,143 \mathrm{~nm}$ aufweist [41].

Die Ergebnisse dieser Anpassungen sind in Tabelle 4.4 für die unterschiedlichen Grenzflächenpositionen aufgelistet.

Für die Grenzflächenposition 1 ist ersichtlich, dass diese Position der Grenzfläche zu keiner guten Anpassung führt, was an einem höheren R-Faktor ersichtlich ist. Diese Grenzflächenposition ist offenbar zu weit in Richtung des amorphen Probenbereiches hineinverschoben (siehe auch Lage der Grenzflächenpositionen in Abb. 4.8).

Dahingegen liegt die Grenzfläche bei Position 4 schon zu weit im kristalli- 


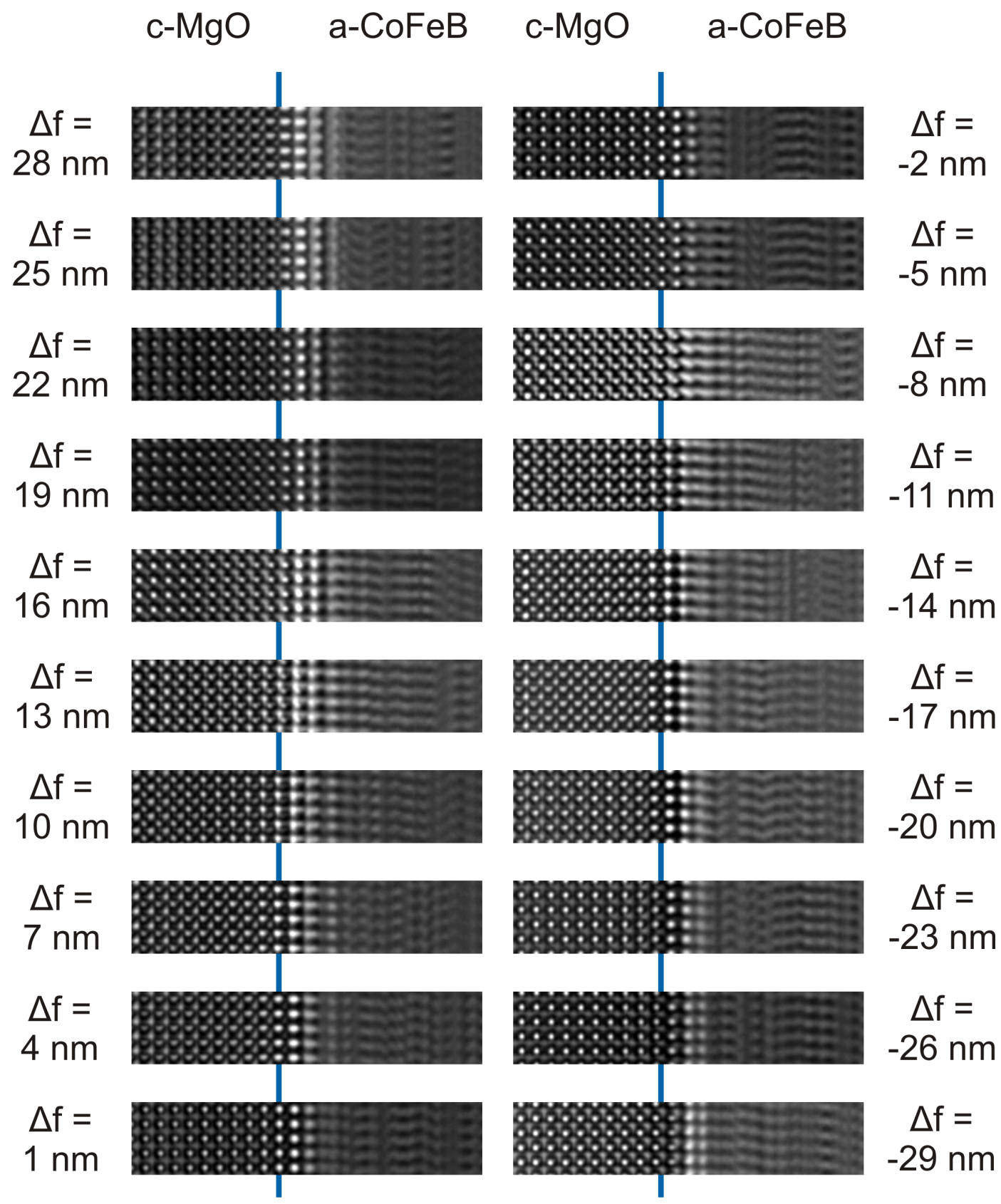

Abbildung 4.9.: Gemittelte Abbildungen der Grenzfläche von $\Delta f=28 n m$ bis $\Delta f=-29 \mathrm{~nm}$. Zur besseren Verdeutlichung wurden die gemittelten Streifen fünfmal untereinander aufgetragen. Die blaue Linie kennzeichnet die festgestellte Position der letzten $\mathrm{MgO}$-Lage. 


\begin{tabular}{|c||l|l|l|l|}
\hline Position & Pos. 1 & Pos. 2 & Pos. 3 & Pos. 4 \\
\hline \hline $\mathrm{R}$ & 885 & 682 & 681 & 739 \\
\hline$d_{a 1}[\mathrm{~nm}]$ & 0,142 & 0,196 & 0,212 & 0,213 \\
\hline$d_{a 2}[\mathrm{~nm}]$ & 0,127 & 0,158 & 0,200 & 0,200 \\
\hline$\sigma_{x 1}$ & 0,052 & 0,038 & 0,018 & 0,015 \\
\hline$\sigma_{y z 1}$ & 0,015 & 0,019 & 0,026 & 0,018 \\
\hline$\sigma_{x 2}$ & 0,069 & 0,246 & 0,036 & 0,014 \\
\hline$\sigma_{y z 2}$ & 0,059 & 0,038 & 0,011 & 0,000 \\
\hline$a_{x}$ & 0,000 & 0,108 & 0,022 & 0,022 \\
\hline$a_{y z}$ & 0,000 & 0,062 & 0,024 & 0,020 \\
\hline
\end{tabular}

Tabelle 4.4: Optimale Parametersätze für die zweidimensionale Verteilungsfunktion nach der iterativen Bildanpassung für verschiedene Grenzflächenpositionen.

nen Teil der Probe. Der R-Faktor steigt ebenfalls an. Auch die $\sigma_{x 1 / y z 1}$ und die $\sigma_{x 2 / y z 2}$ der ersten und zweiten Lage sind klein und entsprechen fast Deltafunktionen, wie dies bei kristallinem Material zu erwarten wäre. Der Abstand $d_{a 1}$ entspricht dem Gitterabstand im kristallinen $\mathrm{MgO}$.

Die Anpassungen an den Grenzflächenpositionen 2 und 3 weisen identische R-Faktoren auf, die deutlich geringer sind als für die Positionen 1 und 4 .

Die Grenzflächenposition 3 weist einen Abstand $d_{a 1}$ auf, der, wie bei Grenzflächenposition 4, identisch ist mit der halbe Gitterkonstanten von $\mathrm{MgO}$ $1 / 2 \cdot d_{M g O}=0,211 \mathrm{~nm}$. Von Burton et al. durchgeführte ,first-principles“-Berechnungen für diesen Abstand in $\mathrm{CoFeB}-\mathrm{MgO}$-Systemen ergaben für diesen Abstand $d_{F e-O}=0,223 \mathrm{~nm}$ bzw. $d_{C o-O}=0,216 \mathrm{~nm}$ für Cobalt terminierte Grenzflächen [24].

Die Grenzflächenposition 2 befindet sich eine Monolage weiter in Richtung des amorphen $\mathrm{CoFeB}$ versetzt. Der Abstand der ersten Co/Fe-Lage zum MgO dieser iterativen Anpassung ergab $d_{a 1}=0,196 \mathrm{~nm}$. Dieser Wert stimmt unter Berücksichtigung des Fehlers mit dem in Abschnitt 4.1.1 bestimmten Abstand von $0,184 \pm 0,02 \mathrm{~nm}$ überein. Dagegen ist jedoch der von Burton et al. berechnete Fe-O-Abstand etwa 14\% größer als der hier vorgefundene. Für reine, massive Eisen(III)-oxid-Strukturen findet man in der Literatur darüber hinaus einen nahezu identischen Abstand von 0,193 nm zwischen $\mathrm{Fe}^{3+}$ und $\mathrm{O}^{2-}$ [108. Die Atomabstände ab der zweiten Lage wurden bei dieser Anpassung mit $d_{a 2}=0,158 \mathrm{~nm}$ bestimmt und liegen etwa $10 \%$ über dem für reines kristallines CoFe nachgewiesenen halben Gitterabstand von 0, $143 \mathrm{~nm}$.

Schlussendlich ist keine eindeutige Aussage über die genaue Grenzflächenpositionen möglich. Dies kann an Grenzflächeninhomogenitäten liegen. Die Ausdehnung des Mittelungsbereiches dieser Untersuchung beträgt aufgrund von sichtbaren Stufen nur 4,2 nm. Die Lamelle ist jedoch fast $11 \mathrm{~nm}$ dick. Es ist 
also sehr wahrscheinlich, dass sich an der Grenzfläche in Strahlrichtung eine Stufe befindet, sodass die oben durchgeführten iterativen Anpassungen zu keinem eindeutigen Ergebnis kommen kann.

In Abbildung 4.10 sind beispielhaft die angepassten simulierten und experimentellen Abbildungen für die Grenzflächenposition 2 dargestellt. Die blaue Linie kennzeichnet die Position der Grenzfläche 2, also die letzte Lage MgO in den simulierten Abbildungen. Sowohl die Abbildungen als auch die die im Anhang befindlichen Profile (siehe C.1 und C.2 zeigen eine gute Übereinstimmung der Maxima- und Minimapositionen im $\mathrm{MgO}$ als auch im CoFeB. Auch die mittleren Intensitäten im kristallinen $\mathrm{MgO}$ und im amorphen $\mathrm{CoFeB}$ passen gut aufeinander. Lediglich einige doppelperiodische Maxima im MgO treten in den experimentellen Profilen nicht so deutlich hervor wie in den simulierten Profilen. In den experimentellen Abbildungen sind diese zum Teil sogar deutlicher zu erkennen als aus den Profilen zu vermuten wäre. Ebenso sind die Kontraste in einigen experimentellen Profilen zum Teil größer als in den simulierten Profilen. Dies ist aufgrund des Stobbs-Faktors in dieser Reihenfolge nicht zu erwarten. Auffällig ist insbesondere in den Profilen, dass die Kontraste im Umfeld der eingezeichneten Grenzflächenposition ( etwa -0,2 nm $\left.<x_{0}<0,2 \mathrm{~nm}\right)$ nicht gut mit den experimentellen Kontrasten übereinstimmen.

Wie oben geschrieben, ist die genaue Lage der Grenzfläche nicht eindeutig bestimmbar. Darüber hinaus zeigen auch die angepassten simulierten Abbildungen an einer der beiden möglichen Grenzflächenpositionen deutliche Kontrastunterschiede an der Grenzfläche, was die Vermutung einer Stufe in Strahlrichtung im angepassten Mittelungsbereich erhärtet. 


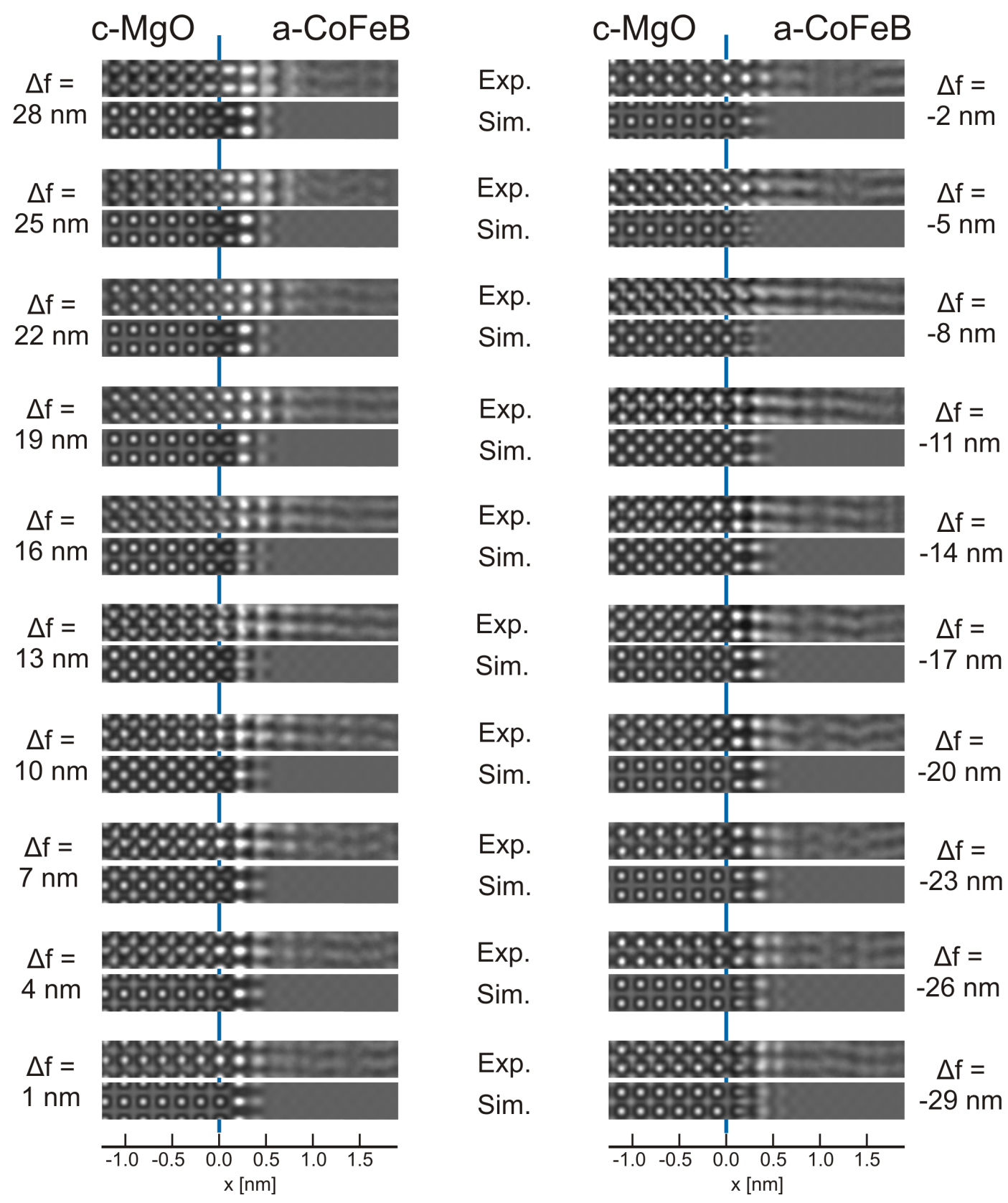

Abbildung 4.10.: Vergleich der simulierten (Unten) und gemittelten experimentellen (Oben) Grenzflächenabbildungen für den Defokusbereich von $\Delta f=28 \mathrm{~nm}$ bis $\Delta f=-29$ nm. Die eingezeichnete Linie kennzeichnet die Grenzflächenposition 2. 


\subsubsection{Einfluss von Grenzflächeninhomogenitäten}

Da aufgrund des R-Faktors keine eindeutige Lage der Grenzflächenposition zu definieren ist und auch die Abbildungen 4.10 und die Intensitätsprofile C.1 und C.2 Hinweise auf Grenzflächeninhomogenitäten geben, wird im Folgenden der Einfluss von Stufen auf die Intensitätsverteilung untersucht.

Die zentrale Frage dabei ist, ob die vorgefundenen experimentellen Kontraste auch ohne eine kristalline Ordnung im CoFeB nur durch Stufeneffekte zu erklären sind. Sollte dies nicht der Fall sein, stellt sich die Frage, ob die Berücksichtigung einer monoatomaren Stufe zu einer besseren Übereinstimmung zwischen experimentellen und simulierten Abbildungen führt und wo und wie diese Stufe in der Lamelle liegt.

Dazu wird die Simulation dahingehend angepasst, dass zum einen Simulationen mit der in Tabelle 4.4 gefundenen kristallinen Ordnung und zum anderen mit einer angenommenen homogenen Atomverteilung im CoFeB durchgeführt werden. Zusätzlich werden zwei verschiedene Arten von Stufen berechnet, hier positive Stufe und negative Stufe genannt. Bei der negativen Stufe fehlt dem kristallinen $\mathrm{MgO}$ in den unteren Scheiben jeweils eine Monolage $\mathrm{MgO}$; bei der positiven Stufe fehlt diese den oberen Scheiben (s. Abb. 4.11). Bei der Simulation mit der kristallinen Grenzflächenordnung wird die Anzahl an Scheiben zwischen den Grenzflächenpositionen 2 und 3 (s. Abb. 4.8) variiert, da bei der Bestimmung der Grenzfläche in Abschnitt 4.1.2.1 beide Positionen einen ähnlichen R-Faktor aufwiesen.

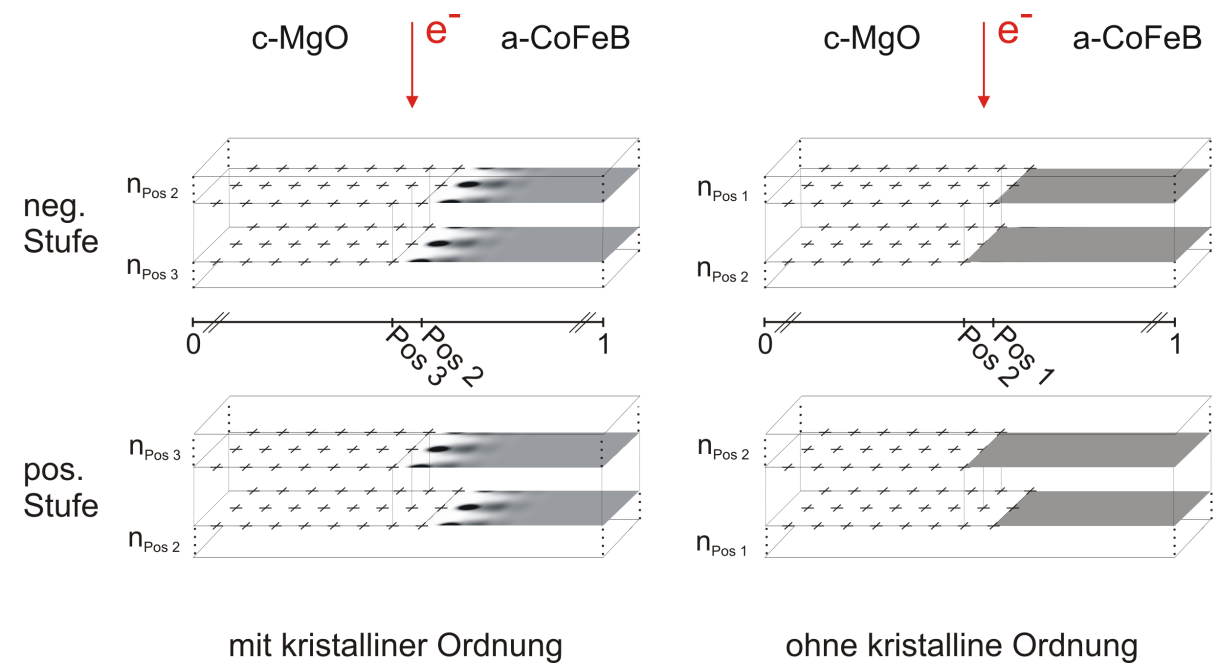

Abbildung 4.11.: Skizze der verschiedenen simulierten Stufen. Links: mit einer kristallinen Ordnung; Rechts: mit homogener Verteilung des CoFeB. 
Unter Annahme einer homogenen Verteilung werden bei der Simulation die Grenzflächenpositionen 1 und 2 genutzt, weil das kristalline $\mathrm{MgO}$ weiter in das amorphe CoFeB hineinragen müsste. Da die Dicke der Probe unverändert ist, beträgt die Gesamtscheibenzahl in allen Simulationen $n=26$.

In Abbildung 4.12 ist das Bildvergleichsmaß R für die unterschiedlichen Stufen und Verteilungen aufgetragen ${ }^{1}$.

Aus diesem Vergleich ist deutlich ersichtlich, dass das Bildvergleichsmaß der simulierten experimentellen Abbildungen unter Annahme einer homogenen Verteilung des CoFeB bis auf eine Rechnung (bei einer negativen Stufe bei $n_{P o s 2}=13$ ) immer größer ist als unter Berücksichtigung einer kristallinen Ordnung. Ebenfalls zeigt sich, dass eine negative Stufe stets bessere Ergebnisse liefert als eine positive Stufe. Bei der negativen Stufe ist ein flaches Minimum mit $R=560$ bei $n_{P o s 2}=16$ vorhanden. Die Berücksichtigung der Stufe führt somit zu einer $11 \%$ besseren Übereinstimmung mit den experimentellen Abbildungen als unter der Annahme einer glatten Grenzfläche bei Position 2.

Dabei gilt zu beachten, dass in den Berechnungen davon ausgegangen wird, dass nur eine Stufe mit einer Monolage entlang der Grenzfläche vorhanden ist. Unter Berücksichtigung mehrerer Stufen beliebiger Größe und Kombinationen

\footnotetext{
${ }^{1}$ Dieser Vergleich ist mit leicht veränderten Kontrastanpassungen erstellt worden und nicht mit dem in Tabelle 4.4 erhaltenen Bildvergleichsmaß $\mathrm{R}$ gegenüber zu stellen. Eine Anpassung der Grenzflächenposition 2 mit den in Tabelle 4.4 angegeben Parametern würde mit diesen Kontrastanpassungen einen R-Faktor von 630 ergeben.
}



Abbildung 4.12:

Vergleich verschiedener Stufen und Stufenpositionen mit und ohne kristalliner Ordnung an der Grenzfläche mittels Bildvergleichsmaß (R). $n_{\text {Posx }}$ gibt die Anzahl der Scheiben mit einer Grenzflächenposition $\quad x$ an. 
würde schnell eine unüberschaubare Kombination an möglichen Realisierungen zustande kommen. Außerdem wird für die Untersuchung des Einflusses der Stufen unter Berücksichtigung einer kristallinen Ordnung diejenige Ordnung genutzt, die aus der Anpassung für die Grenzflächenposition 2 unter Nichtbeachtung der Stufe erzielt wurde.

Für eine genauere Analyse werden im Folgenden iterative Anpassungen der grenzflächeninduzierten Ordnung für verschiedene Stufen durchgeführt. Aus der vorangegangenen Untersuchung geht hervor, dass die hier vorliegende Stufe mit großer Sicherheit eine negative Stufe ist, weshalb diese Voraussetzung in den folgenden Rechnungen angenommen wird und die Anzahl der jeweiligen Scheiben variiert wird. Die Gesamtscheibenzahl beträgt erneut $n=26$. Das Ergebnis (s. Abb. 4.13) zeigt, dass sich wieder ein Minimum in der Mitte der Probe ergibt. Die beste Anpassung mit einem Bildvergleichsmaß von $R=519$ ergibt sich für eine Stufenposition, bei der 13 Scheiben an der Grenzflächenposition 2 liegen und darunter 13 Scheiben an der dritten Grenzflächenposition. Diese Position hat sich also gegenüber der vorherigen Untersuchung zu einer größeren Anzahl an Scheiben in Richtung der dritten Grenzflächenposition verschoben. Dies war zu erwarten, da die vorherige Untersuchung die angepasste grenzflächeninduzierte Ordnung für die Grenzflächenposition 2 genutzt hat. Unter Berücksichtigung der Stufe an dieser Position kann das Bildvergleichsmaß durch die Anpassung der Verteilungsfunktion um weitere $7 \%$ gesenkt
Abbildung 4.13:
Bildvergleichsmaß
(R) nach separater
Anpassung der
Verteilungsfunktion
an verschiedenen
Stufenpositionen.

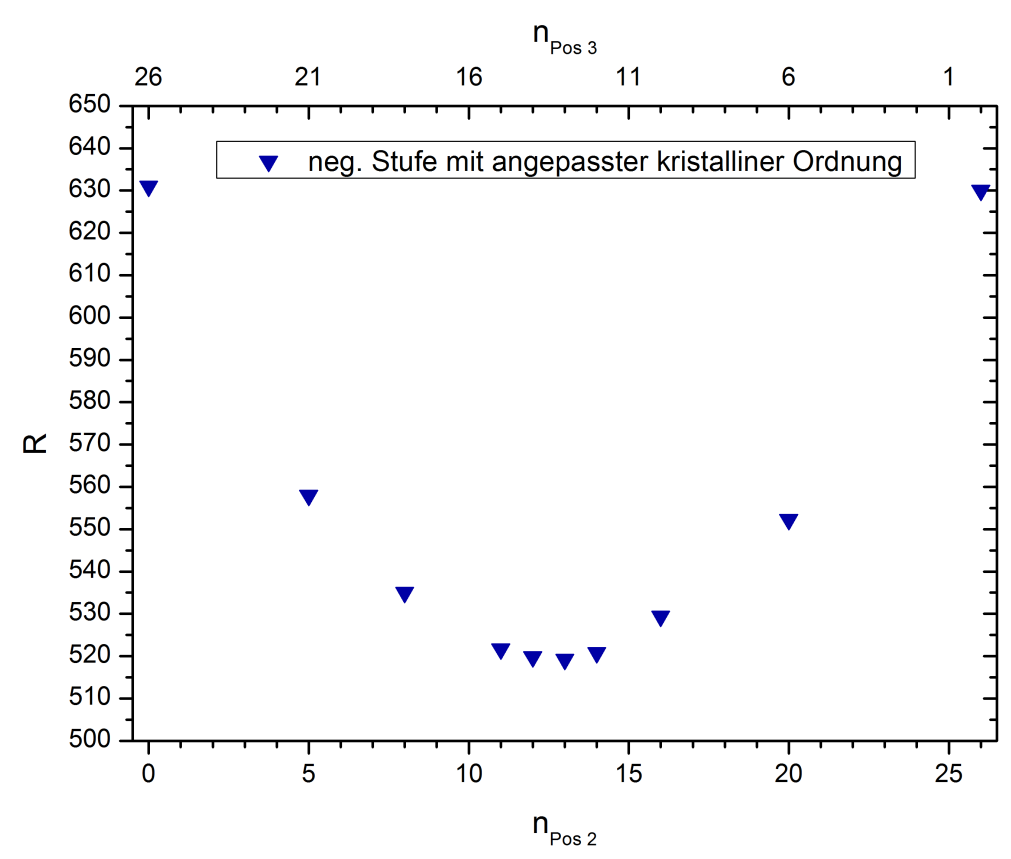


werden. Somit ergibt diese Anpassung unter Berücksichtigung der Stufe eine um insgesamt $18 \%$ bessere Übereinstimmung mit den experimentellen Daten als unter Verwendung der Anpassung ohne Berücksichtigung der Stufe.

In Abbildung 4.14 sind die simulierten und experimentellen Abbildungen dieser iterativen Anpassung gegenübergestellt. Die dazugehörigen Profile befinden sich in den Abbildungen 4.15 und 4.16. Wie in den vorangegangenen Anpassungen stimmen die Intensitätsmaxima und -minima gut mit den experimentellen Daten überein, die Übereinstimmung der absoluten Intensitäten hat sich verbessert, jedoch weisen die experimentellen Daten weiterhin höhere Kontraste auf. Insbesondere in den Profilen ist sichtbar, dass der Kontrast an der Grenzfläch $\AA^{2}$ durch die Berücksichtigung der Stufe besser beschrieben werden kann. Beispielhaft sind in den Abbildungen 4.15 und 4.16 die Positionen an der Grenzfläche mit einem blauen Kreis gekennzeichnet, bei denen sich eine deutliche Verbesserung ergeben hat.

In Tabelle 4.5 sind die iterativ angepassten Parameter für verschiedene Stufenpositionen dargestellt, deren R-Faktoren auch in Abbildung 4.13 aufgetragen sind.

\begin{tabular}{|c||c|c||c|c|c|c||c|c|}
\hline$n_{\text {Pos } 2} / n_{\text {Pos } 3}$ & $5 / 12$ & $8 / 18$ & $11 / 15$ & $12 / 14$ & $13 / 13$ & $14 / 12$ & $16 / 10$ & $20 / 6$ \\
\hline $\mathrm{R}$ & 558 & 535 & 522 & 520 & 519 & 521 & 530 & 552 \\
\hline$d_{a 1}[\mathrm{~nm}]$ & 0.211 & 0.212 & 0.212 & 0.213 & 0.212 & 0.213 & 0.214 & 0.214 \\
\hline$d_{a 2}[\mathrm{~nm}]$ & 0.209 & 0.207 & 0.210 & 0.210 & 0.210 & 0.210 & 0.209 & 0.196 \\
\hline$\sigma_{x 1}$ & 0.026 & 0.025 & 0.026 & 0.024 & 0.024 & 0.024 & 0.031 & 0.034 \\
\hline$\sigma_{y z 1}$ & 0.023 & 0.024 & 0.028 & 0.023 & 0.025 & 0.027 & 0.026 & 0.030 \\
\hline$\sigma_{x 2}$ & 0.046 & 0.047 & 0.051 & 0.058 & 0.056 & 0.061 & 0.064 & 0.078 \\
\hline$\sigma_{y z 2}$ & 0.029 & 0.035 & 0.033 & 0.034 & 0.034 & 0.035 & 0.039 & 0.041 \\
\hline$a_{x}$ & 0.010 & 0.011 & 0.016 & 0.018 & 0.019 & 0.021 & 0.016 & 0.022 \\
\hline$a_{y z}$ & 0.007 & 0.009 & 0.018 & 0.014 & 0.020 & 0.018 & 0.008 & 0.015 \\
\hline
\end{tabular}

Tabelle 4.5.: Ergebnisse der iterativen Bildanpassung für verschiedene Grenzflächenpositionen. Die angepassten Parameter im Bereich $n_{P o s 2} / n_{\text {Pos } 3}=11 / 15-14 / 12$ weisen keine signifikanten Unterschiede auf.

Die Anpassung mit der Stufenposition $n_{P o s 2} / n_{P o s 3}=13 / 13$ ergab mit einem R-Faktor von 519 die beste Übereinstimmung. Der Parameter $d_{a 1}$ ist bei dieser Anpassung unter Berücksichtigung der Stufen mit $d_{a 1}=0,212 \mathrm{~nm}$ um $15 \%$ größer als der Abstand, der an der kristallin/kristallinen Grenzfläche

\footnotetext{
${ }^{2}$ Durch die Stufen ist die Grenzfläche nicht mehr klar definiert. Deshalb wird hier für die bessere Vergleichbarkeit mit den vorherigen Ergebnissen die gekennzeichnete Grenzfläche weiterhin bei der Grenzflächenposition 2 mit $x=0 \mathrm{~nm}$ bzw. der blauen Linie gekennzeichnet.
} 


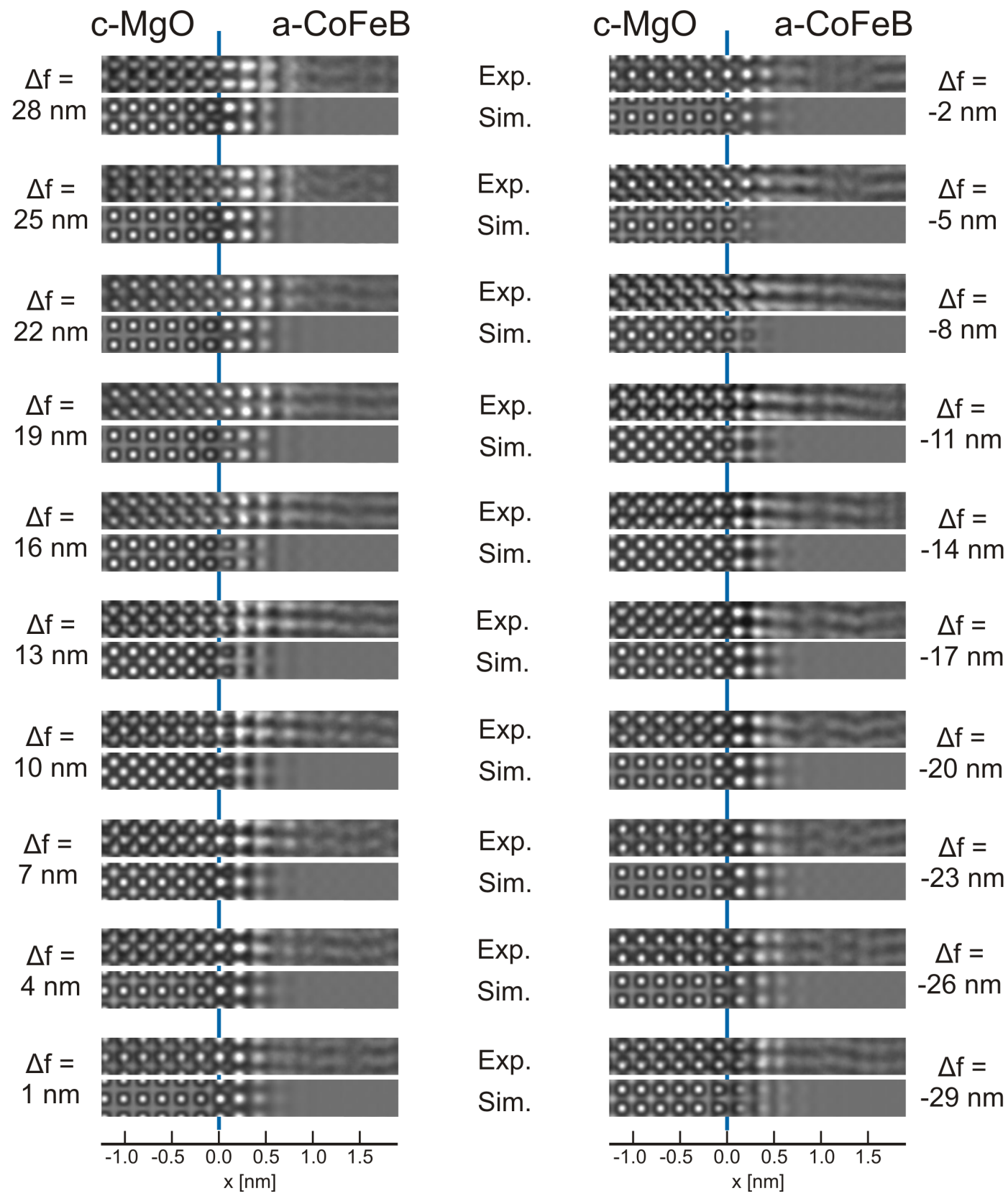

Abbildung 4.14.: Darstellung der experimentellen Grenzflächenabbildungen im Vergleich zu den simulierten Abbildungen unter Berücksichtigung einer Grenzflächenstufe. Die blaue Linie kennzeichnet die Grenzflächenposition 2, die Grenzflächenposition 3 liegt eine Atomlage weiter links. 

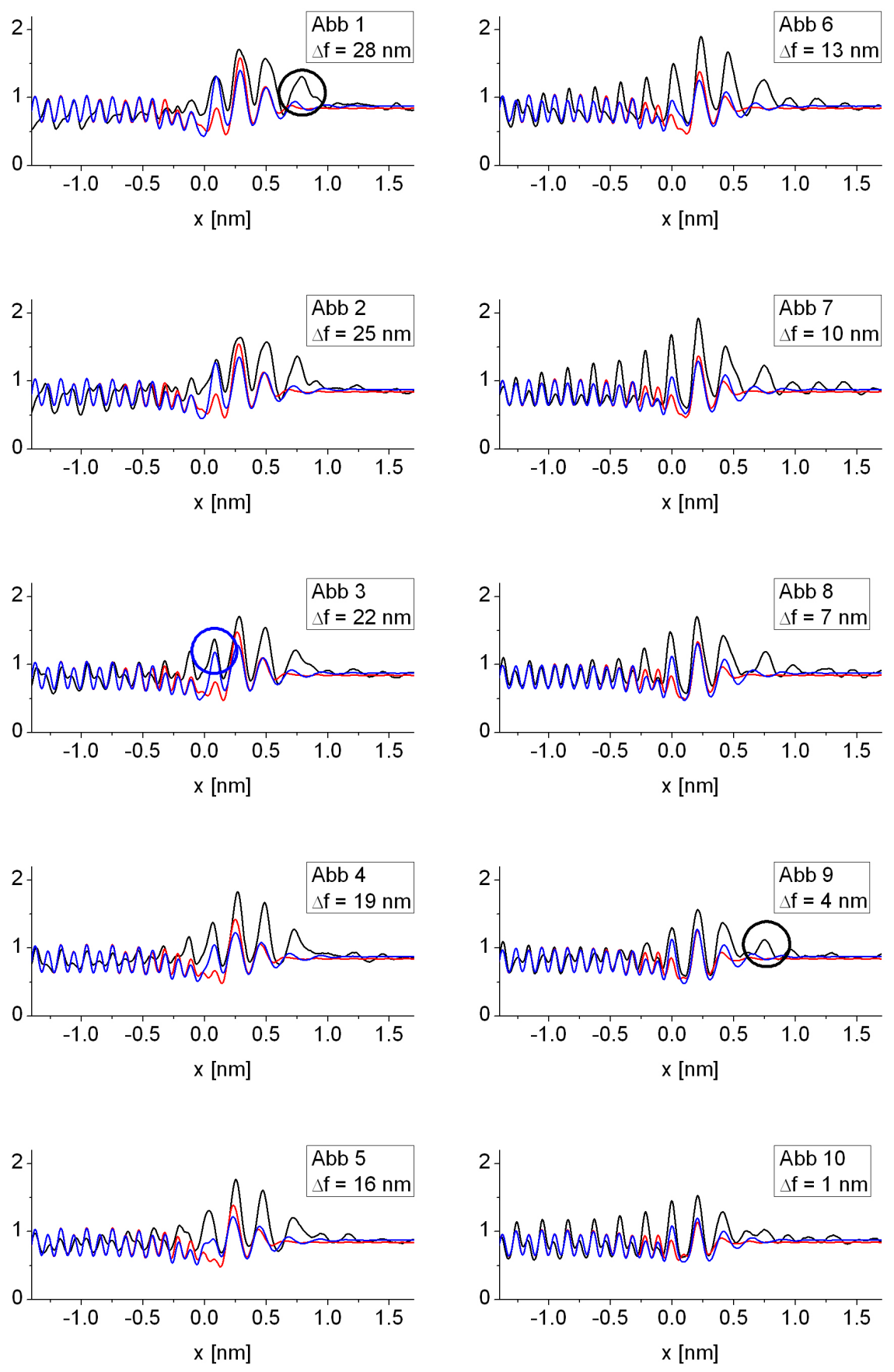

- Exp ohne Stufe - mit Stufe

Abbildung 4.15.: Vergleich der Intensitätsprofile der Abbildungen 1-10 für die beste Verteilungsfunktion unter Berücksichtigung einer Stufe (blau) mit den experimentellen Intensitäten (schwarz) und zu den Intensitätsprofilen ohne Berücksichtigung einer Stufe (rot) aus Abschnitt 4.1.2.1. 



- Exp - ohne Stufe - mit Stufe

Abbildung 4.16.: Vergleich der Intensitätsprofile der Abbildungen 11-20 für die beste Verteilungsfunktion unter Berücksichtigung einer Stufe (blau) mit den experimentellen Intensitäten (schwarz) und zu den Intensitätsprofilen ohne Berücksichtigung einer Stufe (rot) aus Abschnitt 4.1.2.1. 
gefunden wurde. Im Vergleich mit den in der Literatur dargestellten Werten liegt der hier ermittelte Abstand geringfügig darunter. So befindet sich der von Burton et al. [24] berechnete Wert für MgO/CoFeB-Systeme mit $d_{F e-O}=$ $0,223 \mathrm{~nm} 5 \%$ über dem hier gefundenen. Ebenfalls sind Messungen von Wang et al. [113, in denen der Abstand $d_{F e-O}=0,220 \mathrm{~nm}$ zwischen $\mathrm{O}$ und Fe in $\mathrm{MgO} / \mathrm{Fe}-S y s t e m e n$ untersucht wurde, $3,8 \%$ größer. Am nächsten kommt der hier ermittelte Wert dem von Butler et al. [7] berechneten Wert für $\mathrm{MgO} / \mathrm{Fe}-$



Der Parameter $d_{a 2}$ darf in dieser Anpassung Werte zwischen 0,08 nm $\leq$ $d_{a 2} \leq 0,21 \mathrm{~nm}$ annehmen. Der Parameter $d_{a 2}$ ist in dieser Rechnung signifikant größer. Vermutlich rührt das aus der sich überlappenden Verschmierung der Atompositionen in x-Richtung ab der zweiten Lage, welche durch den Algorithmus besser angepasst werden konnte.

Dazu weist $\sigma_{x 2}$, der die Verschmierung in x-Richtung für die zweite Lage angibt, einen kleineren Wert auf als in der Anpassung für die Grenzflächenposition 2 (siehe Tabelle 4.4). Somit wird bei dieser Anpassung das Maximum in der atomaren Dichte durch ein stärker lokalisiertes Maximum beschrieben als durch zwei stärker verschmierte, die dafür enger beieinander liegen. Der ermittelte Wert für $\sigma_{y z 2}$ ist, verglichen mit der Anpassung ohne Berücksichtigung einer Stufe, nahezu unverändert.

In Abbildung 4.17a ist die atomare Dichte für eine Anpassung an die Stufenposition $n_{\text {Pos } 2} / n_{\text {Pos } 3}=13 / 13$ dargestellt. Hier ist gut zu sehen, dass eine durch das kristalline $\mathrm{MgO}$ induzierte Ordnung an der Grenzfläche existiert. Die erste Lage $\mathrm{Co} / \mathrm{Fe}$ ist noch deutlich lokalisierter als die folgenden. Die zweite Lage $\mathrm{Co} / \mathrm{Fe}$ ist schon ,ausgeschmierter“. Somit nimmt die Ordnung mit steigendem Abstand zur Grenzfläche ab und resultiert letztendlich in einer homogenen Verteilung.

Die Ausdehnung des vom kristallinen Teil beeinflussten Übergangsbereiches des CoFeB wird analog zum Vorgehen bei Thiel [21] und Borgardt et al. [51] bestimmt. Dazu wird ab einem bestimmten Punkt $x$ die berechnete zweidimensionale Verteilungsfunktion durch eine homogene Verteilung ersetzt und anschließend die daraus resultierenden, simulierten Abbildungen mit den gemittelten experimentellen Abbildungen verglichen. Unterhalb einer Ausdehnung der Verteilungsfunktion von etwa $x=0,7 \mathrm{~nm}$ steigt der R-Faktor stark an (s. Abb 4.17b), weshalb dieser Punkt als Ende des Übergangsbereiches definiert wird. Dies entspricht etwa 3-4 Atomlagen CoFe.

Aus der Tabelle 4.5 lässt sich auch auf die Empfindlichkeit der angepassten Parameter auf die Stufenposition schließen. Besonders bei den Stufenpositionen $n_{\text {Pos } 2} / n_{\text {Pos } 3}=11 / 15-14 / 12$ unterscheiden sich die angepassten Parameter und somit auch die zweidimensionalen Verteilungsfunktionen nicht signifikant. 


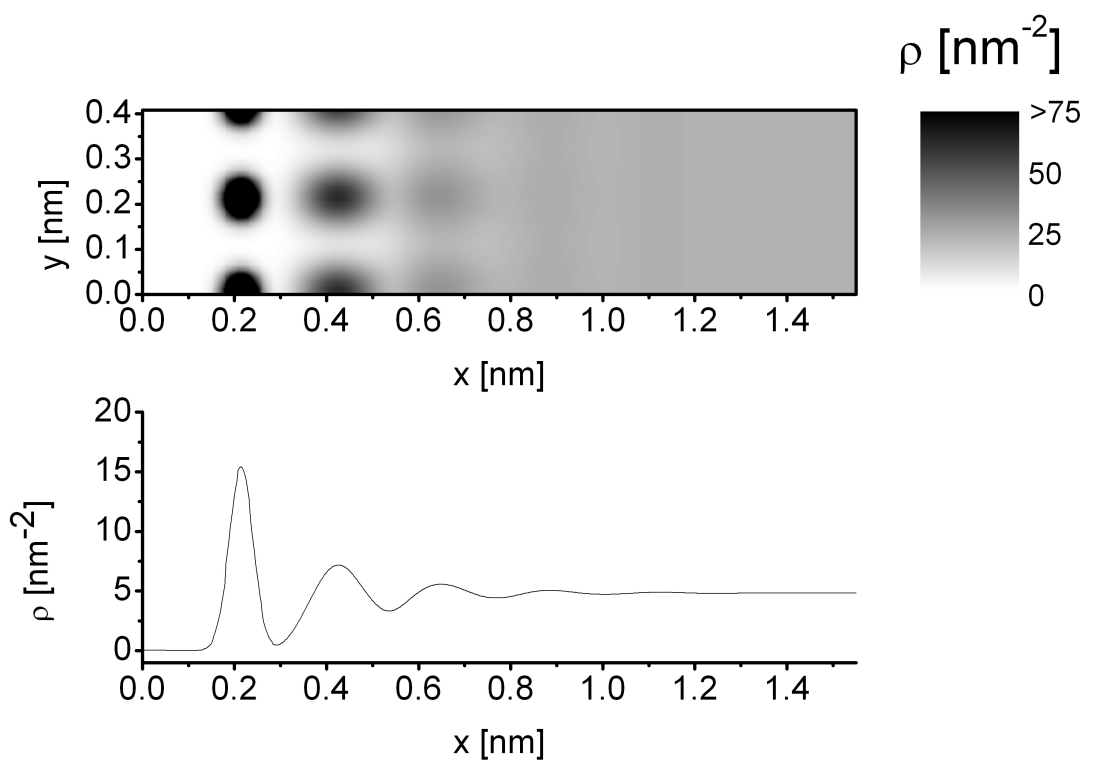

(a)

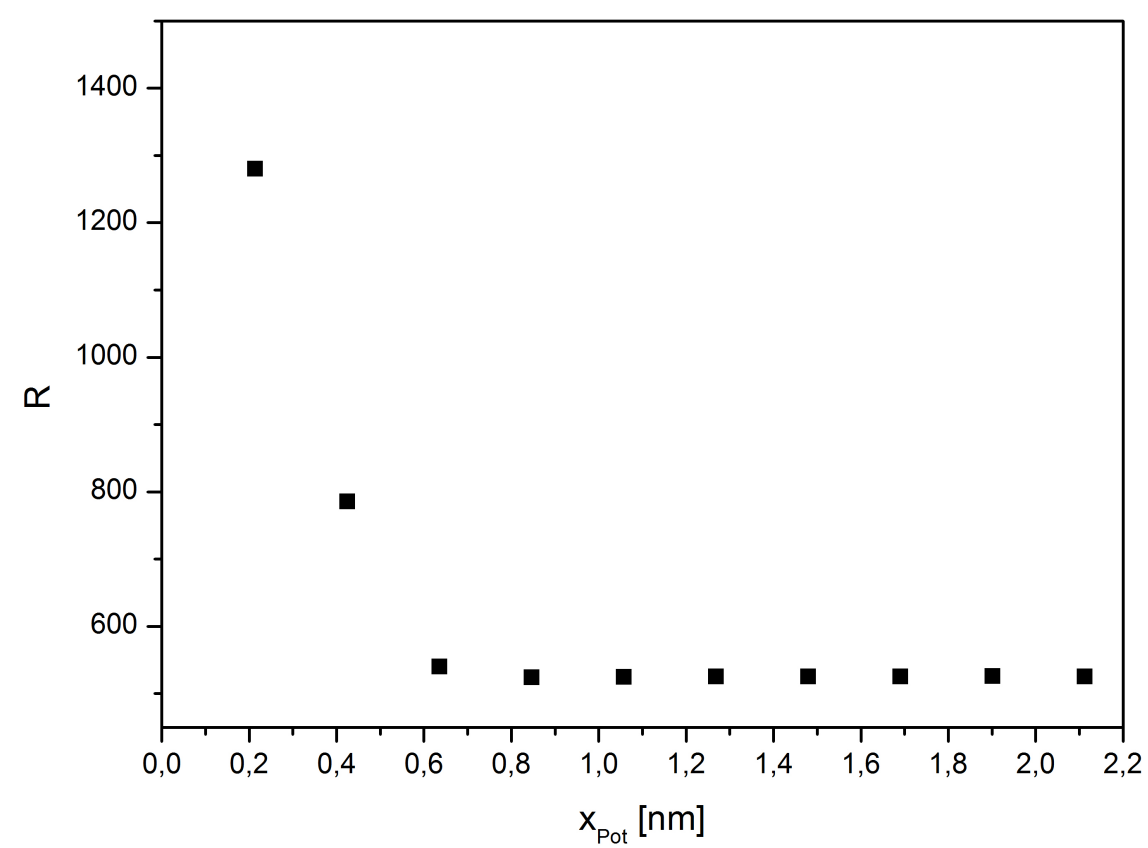

(b)

Abbildung 4.17.: a) Oben: Darstellung der berechneten zweidimensionalen Verteilungsfunktion unter Berücksichtigung der Stufe (oben). Profil der zweidimensionalen Verteilungsfunktion (unten). b) Bestimmung der Ausdehnung des beeinflussten Übergangsbereiches zwischen kristallinem und amorphem Bereich: Aufgetragen ist der $R$ Faktor $(R)$ für verschiedene Ausdehnungen des projizierten Potentials ( $\left.x_{\text {Pot }}\right)$. 


\subsubsection{Iterative Anpassung des strukturellen Übergangs mit 10 Parametern}

In den Profilen der Abbildungen 4.15 und 4.16 als auch in der Abbildung 4.14 sind Kontraste bei $x \geq 0,7 \mathrm{~nm}$ sichtbar (schwarze Kreise in Abb. 4.15 und Abb. 4.16 ), bei denen man von einer systematischen Ordnung ausgehen könnte, die mit den acht Parametern der Anpassung in Abschnitt 3.3.4, nicht besser beschrieben werden konnte. Um eine von den höheren Lagen unabhängige Anpassung der Parameter $\sigma_{x 2}$ und $\sigma_{y z 2}$ zu ermöglichen, wird die Anpassung im Folgenden um zwei Parameter $\sigma_{x 3}$ und $\sigma_{y z 3}$ erweitert. Dies soll zu einer besseren Anpassung der weiteren Lagen führen. Die Anpassung mit diesen 10 Parametern wird unter Berücksichtigung der Stufe aus Abschnitt 4.1.2.2 an der Position $n_{\text {Pos } 2} / n_{\text {Pos } 3}=13 / 13$ durchgeführt. Tabelle 4.6 listet die durch diese Anpassung erhaltenen Parameter auf. Die Anpassung mit zehn Parametern bestätigt im Großen und Ganzen die für acht Parameter erhaltene Anpassung aus Abschnitt 4.1.2.2 (s. Tab. 4.5). Insbesondere die Parameter $d_{a 1}$ und $d_{a 2}$, weichen weniger als $3 \%$ von den dort bestimmten Werten ab. Diese nur geringfügigen Änderungen schlagen sich auch in einem nur um $<1 \%$ verbesserten R-Faktor von 516 nieder.

\begin{tabular}{|c||l|}
\hline $\mathrm{R}$ & 516 \\
\hline$d_{a 1}[\mathrm{~nm}]$ & 0.213 \\
\hline$d_{a 2}[\mathrm{~nm}]$ & 0.205 \\
\hline$\sigma_{x 1}$ & 0.024 \\
\hline$\sigma_{y z 1}$ & 0.029 \\
\hline$\sigma_{x 2}$ & 0.059 \\
\hline$\sigma_{y z 2}$ & 0.023 \\
\hline$\sigma_{x 3}$ & 0.085 \\
\hline$\sigma_{y z 3}$ & 0.055 \\
\hline$a_{x}$ & 0.022 \\
\hline$a_{y z}$ & 0.027 \\
\hline
\end{tabular}

Tabelle 4.6: $\quad E r$ gebnisse des besten Parametersatzes der iterativen Bildanpassung mit zehn Parametern.

Aus den sich im Anhang befindlichen Profilen C.4 und C.5, die auch den direkten Vergleich zwischen den in Abschnitt 4.1.2.2 bestimmten Profilen zeigen, wird deutlich, dass es zu keiner signifikant besseren Übereinstimmung der Intensitäten bei höheren Lagen kommt.

Die erhaltene zweidimensionale Verteilungsfunktion für zehn Parameter (s. Abb. C.6a) und auch die daraus resultierende Reichweite des strukturellen Übergangs zwischen kristallinem $\mathrm{MgO}$ und amorphem $\mathrm{CoFeB}$ (s. Abb. C.6b) weisen keine Unterschiede zu den in Abschnitt 4.1.2.2 bestimmten auf. Somit 
bringen weitere Freiheitsgrade bei der Konstruktion der Verteilungsfunktion dieses Systems keine bessere Übereinstimmung mit den experimentellen Kontrasten. Daher ist davon auszugehen, dass diesen Kontrasten über $x=0,7 \mathrm{~nm}$ keine systematische Struktur zugrunde liegt.

\subsubsection{Zusammenfassende Diskussion der Untersuchung des strukturellen Übergangs der c-MgO/a-CoFeB Grenzfläche}

Die Untersuchungen des strukturellen Übergangs zwischen amorphem CoFeB und kristallinem $\mathrm{MgO}$ zeigen, dass dieser sich gut mit dem vorgestellten IDSIMA-Verfahren untersuchen lässt. Die simulierten Abbildungen und Profile zeigen eine gute Übereinstimmung der Maxima- und Minimapositionen mit den experimentellen Abbildungen und Profilen. Bei der iterativen Anpassung ist dabei weniger entscheidend, ob diese mit zehn oder acht Parametern durchgeführt wurde; wichtiger ist die Berücksichtigung von Grenzflächeninhomogenitäten. Unter deren Berücksichtigung kann eine um $18 \%$ bessere Anpassung erreicht werden. Es zeigte sich, dass in dem hier untersuchten Ausschnitt der Grenzfläche die Stufe mittig in der Lamelle liegt. Dabei ragt das kristalline $\mathrm{MgO}$, welches in z-Richtung gesehen oben liegt, eine Monolage weiter in das $\mathrm{CoFeB}$ hinein als die darunterliegende MgO-Schicht. Diese Situation entspricht in der Abbildung 4.11 der Stufe links oben. Dabei sind beide Schichten jeweils $5,5 \mathrm{~nm}$ dick, was 13 Scheiben entspricht. Die Berücksichtigung einer Stufe in der Lamelle kann zwar einige Kontrastunterschiede in unmittelbarer Nähe der Grenzfläche $(0 \leq x \leq 0.25 \mathrm{~nm})$ erklären (s. Abb.4.18), aber die Kontraste in den simulierten Abbildungen sind trotz allem noch immer geringer als in den experimentellen Abbildungen. Ursächlich könnten weitere Stufen an der Grenzfläche sein, in denen das $\mathrm{MgO}$ noch weiter in Richtung $x>0 \mathrm{~nm}$ verschoben ist. Aufgrund der Stufendichte ist auf den $11 \mathrm{~nm}$ dieser Lamelle jedoch nicht mit einer deutlich größeren Anzahl an Stufen zu rechnen. Des Weiteren könnten beim Dünnen der Lamelle geringe Dickenvariationen an der Grenzfläche aufgrund unterschiedlicher Sputterraten aufgetreten sein. Dies würde zu einer Änderung des kristallinen Musters an der Grenzfläche führen, das wiederum einen stärkeren Kontrast zur Folge haben könnte.

Aus den iterativen Anpassungen an verschiedene Stufenpositionen lässt sich zudem erkennen, dass sich die angepassten Parameter und somit die zweidimensionale Verteilungsfunktion bei kleinen Verschiebungen der Stufenposition nicht signifikant ändern.

Der Abstand zwischen dem letzten Sauerstoffatom im $\mathrm{MgO}$ und dem ersten $\mathrm{Co} /$ Fe-Atom wurde hier mit $d_{a 1}=0,212 \mathrm{~nm}$ bestimmt, welcher $15 \%$ größer ist als der unter Abschnitt 4.1.1.1 bestimmte Abstand an der c-MgO/c- 


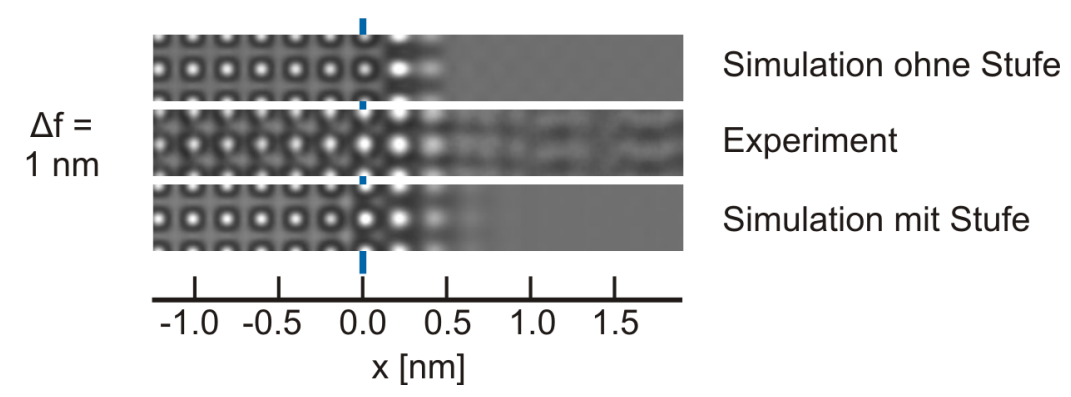

\begin{abstract}
Abbildung 4.18.: Vergleich der experimentellen Abbildung (Mitte) bei $\Delta f=1 \mathrm{~nm}$ mit den korrespondierenden, simulierten Abbildungen ohne Berücksichtigung einer möglichen Stufe (oben) und unter Berücksichtigung einer Stufe (unten): Gut sichtbar ist die bessere Übereinstimmung des Kontrastes unter Berücksichtigung der Stufe an der Grenzfläche (blaue Linie).
\end{abstract}

CoFe-Grenzfläche. Verglichen mit Berechnungen, die Burton et al. für dieses Materialsystem durchführten, ist der hier gefundene Abstand $5 \%$ kleiner als der von Burton bestimmte Fe-O-Abstand von $d_{F e-O}=0,223 \mathrm{~nm}$. Der ebenfalls von Burton et al. berechnete Co-O-Abstand ist mit $d_{C o-O}=0,216 \mathrm{~nm}$ dagegen weniger als $2 \%$ größer. Weiterhin zeigten die Berechnungen von Burton, dass es für Bor energetisch günstiger ist an der CoFe-MgO-Grenzfläche zu verbleiben als sich im bulk c-CoFe zu befinden. Dieses zurückgebliebene Bor an der Grenzfläche weist einen großen Einfluss auf die Bindungsenergien an der Grenzfläche auf. So führt das Bor zu einer Schwächung der O-Co- bzw. $\mathrm{O}-\mathrm{Fe}-$ Bindung, da es zu einer bevorzugten Bindung zwischen Fe-B bzw. CoB kommt [24]. Diese schwächere Bindung zwischen O-Co und O-Fe sollte zu einem längeren Bindungsabstand führen, der hier aber nicht gefunden wurde.

Neben CoFeB-MgO-Systemen gibt es auch Untersuchungen von Fe-MgOSystemen: So konnten Wang et al. [113] einen $d_{F e-O}$ Abstand von 0,220 nm $(+4 \%$ zu dem hier gefunden Abstand) experimentell bestimmen, der von Butler et al. [7] in Berechnungen mit $d_{F e-O}=0,2169 \mathrm{~nm}(+2 \%)$ bestätigt werden konnte. In Fe-MgO-Systemen wurden auch Untersuchungen an oxidierten Grenzflächen durchgeführt. Bei oxidierten Grenzflächen liegt zwischen den FeAtomen der ersten Lage Fe eine Monolage Sauerstoff (s. Abb. 4.19). Die an diesen Grenzflächen experimentell gefundenen und berechneten Abstände sind jedoch größer als die an nicht oxidierten Grenzflächen. So wurde von Meyerheim et al. [114, 115] ein Abstand $d_{F e-O}=0,235 \mathrm{~nm}(+11 \%)$ gemessen und von $\mathrm{Yu}$ et al. ein Abstand von 0,253 nm (+19\%) per ab initio Berechnungen bestimmt 116].

Der quantitative Vergleich der aus 20 Streifen gemittelten, experimentellen Defokusserie mit simulierten elektronenmikroskopischen Abbildungen ba- 
Abbildung 4.19: Eine oxidierte $\mathrm{MgO}-\mathrm{CoFe}$ Grenzfläche enthält eine zusätzliche Sauerstofflage in der ersten $\mathrm{Fe} / \mathrm{Co}$ Schicht.

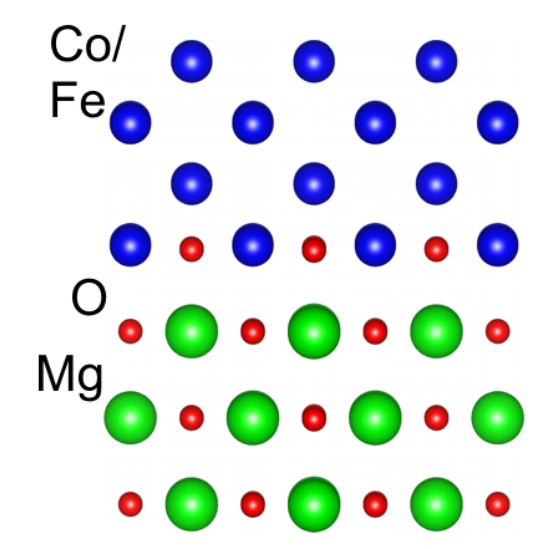

sierend auf einer zweidimensionalen Verteilungsfunktion zeigt, dass die Ausdehnung des Übergangsbereiches bei einem nicht ausgelagerten System etwa 0,7 nm beträgt. Dies entspricht ungefähr 3-4 kristallinen Monolagen CoFe an der Grenzfläche. Die hier gefundenen $\approx 0,7 \mathrm{~nm}$ sind im Vergleich zu den von Thiel ermittelten 1,4 nm nur halb so groß [53, 21]. Im Gegensatz zu dem hier untersuchten metallischen CoFe handelt es sich bei dem von Thiel untersuchten c-Si/a-Ge bzw. c-Si/a-Si um ein kovalent gebundenes Materialsystem bei dem aufgrund von gerichteten Bindungen auch ein größerer Wert zu erwarten ist. 


\subsection{Bor-Diffusion}

Ein kristalliner Übergang zwischen dem $\mathrm{MgO}$ und dem CoFe ist nicht die einzige Voraussetzung zum Erzielen hoher TMR-Werte in MTJs. Das herausdiffundieren des Bors aus dem CoFeB beim Auslagern ist eine Voraussetzung für die Kristallisation dieser Schicht, weshalb dieser, wie Abschnitt 2.2.2.2 und 2.2.2.1 schon verdeutlichten, ebenfalls eine große Bedeutung zukommt. Erst durch das Ermöglichen einer Kristallisation des $\mathrm{CoFeB}$ am $\mathrm{MgO}$ kommt es zu einer Ausbildung des Symmetriefiltereffekts, der zu hohen TMR-Werten in den MTJs führt. Beim Auslagern besteht jedoch ebenfalls die Möglichkeit einer Kristallisation an der Deckschicht, was die Kristallisation am MgO und somit die Bildung dieses Symmetriefilters verhindert. Außerdem kann das herausdiffundierende Bor sich in oder an der MgO-Barriere ablagern, was zudem die Leitung durch das $\Delta_{1}$-Band reduziert. In den beiden folgenden Abschnitten sollen die Einflüsse der Deckschicht (Abschnitt 4.2.1) und der Herstellungsweise der MgO-Tunnelbarriere (Abschnitt 4.2.2) auf die Bor-Diffusion untersucht werden.

\subsubsection{Einfluss der Deckschicht auf die Bor-Diffusion und das Kristallisationsverhalten}

In diesem Abschnitt werden die Einflüsse des Deckschichtmaterials auf die BorDiffusion und das Kristallisationsverhalten von CoFeB beim Auslagern untersucht. Diese Untersuchung erfolgt an Modellsystemen, deren unterschiedlicher Aufbau in Abbildung 4.20 skizziert sind. Im Gegensatz zu funktionsfähigen MTJs lassen sich in diesen Modellsystemen die Schichtdicken freier wählen und Beeinflussungen anderer Materialien, die sich in MTJs finden weitestgehend ausschließen. Die genauen Eigenschaften und deren Herstellung befinden sich im Anhang in Abschnitt B.3.

Die vier auf $\mathrm{MgO}$ gewachsenen Proben weisen 5 bzw. 100 nm dicke CoFeBSchichten auf und sind jeweils mit einer Deckschicht versehen. Als Deckschichtmaterial finden Ta und $\mathrm{Ru}$ weit verbreitete Anwendung in MTJs mit hohen TMR 2], weshalb diese Deckschichtmaterialien auch im Folgenden untersucht werden. Alle Proben werden für 60 Minuten bei $450{ }^{\circ} \mathrm{C}$ ausgelagert, um eine Kristallisation des zu untersuchenden CoFeB (s. Abschnitt 2.2.2.1) zu ermöglichen.

Da die Kristallisation des CoFeB sowohl am MgO als auch an der Pufferund Deckschicht möglich ist, ist bei den Proben mit der $100 \mathrm{~nm}$ dicken Schicht CoFeB eine getrennte Untersuchung dieser beiden Kristallisationsorte möglich, 




MgO



- CoFeB



Ta



$\mathrm{Ru}$

Abbildung 4.20.: Übersicht der auf MgO-Substrat hergestellten Proben mit 5 bzw. 100 nm dicker CoFeB-Schicht und darüberliegender Deckschicht aus Ta oder Ru.

sofern die CoFeB-Schicht nicht durchgängig kristallisiert ist.

Die TEM-Abbildung 4.21 zeigt die $100 \mathrm{~nm}$ dicken CoFeB-Schicht-Proben mit der Ru-Deckschicht (a) und der Ta-Deckschicht (b). In den TEM-Abbildungen ist eine gute Unterscheidung des kristallisierten CoFe (dunklerer Kontrast) und des amorphen $\mathrm{CoFeB}$ (hellerer Kontrast) möglich. In beiden Proben ist eine kristalline Schicht CoFe sichtbar, welche epitaktisch auf dem $\mathrm{MgO}$ gewachsen ist. Diese beträgt im Fall der Ru-Deckschichtprobe $13 \mathrm{~nm}$ und ist bei der Ta-Deckschichtprobe mit $25 \mathrm{~nm}$ deutlich dicker, was auf eine schnellere Kristallisation im Fall der Ta-Deckschicht hinweist. Jedoch befindet sich in der Ru-Deckschichtprobe an der Grenzfläche zum polykristallinen hcp-Ru ebenfalls eine $25 \mathrm{~nm}$ dicke Schicht polykristallines CoFe. Eine Kristallisation des CoFeB am nanokristallinen Ta findet dagegen nicht statt.

EDX-Punktmessungen in der amorphen CoFeB-Schicht zur Bestimmung des Fe:Co-Verhältnisses ergeben ein Verhältnis von 29(1):71(1). Da es sich beim Sputter Target um eine $\left(\mathrm{Co}_{25} \mathrm{Fe}_{75}\right)_{80} \mathrm{~B}_{20}$-Legierung handelt, ist die CoFeBSchicht Co-reicher als erwartet. Dieses oft beobachtete Verhalten ist auf eine leicht höhere Sputterrate des Cobalts zurückzuführen [50].

In den Proben mit $100 \mathrm{~nm}$ dicken CoFeB-Schichten befinden sich an der Grenzfläche zum MgO ausgedehnte kristallisierte CoFe-Schichten. Daher ist zu erwarten, dass diese Schichten in den Proben mit nur $5 \mathrm{~nm}$ dicken CoFeBSchichten vollständig kristallisiert sind (s. Abb. 4.22). 


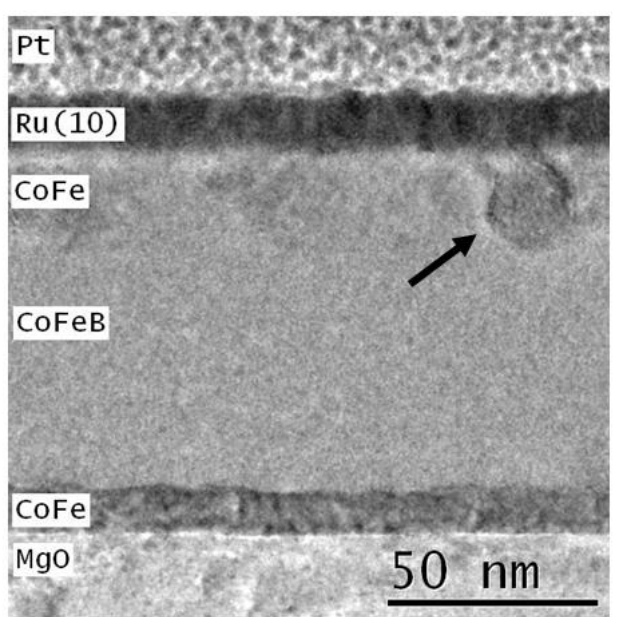

(a)



(b)

\begin{abstract}
Abbildung 4.21.: TEM-Abbildungen von ausgelagerten Proben mit 100 nm dicker CoFeB-Schicht: a) Die Probe mit Ru-Deckschicht zeigt eine etwa 13 nm dicke Schicht kristallinen CoFe an der Grenzfläche zum $\mathrm{MgO}$ und eine Kristallisation an der Deckschicht b) Die Kristallisation an der Grenzfläche zum $\mathrm{MgO}$ ist bei der TaDeckschichtprobe mit $25 \mathrm{~nm}$ dicker; dafür tritt hier keine Kristallisation zur Deckschicht hin auf. [117]
\end{abstract}


Das gilt auch für die Ta-Deckschichtprobe (Abb. 4.22b und 4.22d). Das CoFeB ist vollständig vom $\mathrm{MgO}$ aus in bcc(001)-CoFe kristallisiert. Darüber schließt sich das nanokristalline Ta an.

Im direkten Vergleich der Ru-Deckschichtprobe mit der Ta-Deckschichtprobe fällt auf, dass in der Ru-Probe die kristallisierte CoFe-Schicht dünner ist als in der Ta-Probe. Außerdem ist in der Ru-Deckschichtprobe eine dünne, vom Kontrast hellere, amorphe Zwischenschicht sichtbar, die zwischen der kristallisierten CoFe- und der darüberliegenden polykristallinen hcp-Ru-Schicht liegt.

Da die Kristallisation des CoFeB maßgeblich durch das Herausdiffundieren des Bors beeinflusst wird, werden im Folgenden EELS-Untersuchungen an den Proben mit der $5 \mathrm{~nm}$ dicken CoFeB-Schicht durchgeführt, um nach dem Auslagern Unterschiede der Segregation von Bor festzustellen. Die EELSUntersuchungen wurden im FEI TITAN ETEM unter STEM-Bedingungen durchgeführt, um einen direkten Zusammenhang des Probenortes mit den erhaltenen EELS-Spektren zu erlangen. Der Strahldurchmesser beträgt dabei etwa $1,5 \AA$ und hat einen Semikonvergenzwinkel von $\sim 9,5 \mathrm{mrad}$. Die Spektrometerdispersion liegt bei $0,25 \mathrm{eV} / \mathrm{Kanal}$, was in einer Energieauflösung von 1,5 eV resultiert und ein $512 \mathrm{eV}$ breites Energiespektrum auf dem Detektor erzeugt. Dieses ausgedehnte Energiespektrum ist erforderlich, um simultan das Signal der Bor-K-Absorptionskante bei $188 \mathrm{eV}$ und der Sauerstoff-KAbsorptionskante bei $532 \mathrm{eV}$ aufzunehmen [118]. Um Schädigungen der Probe durch den Elektronenstrahl zu verringern, wird das EELS-Spektrum an meh-


griert.

Abbildung 4.23 zeigt für die Messung an der Ru-Deckschichtprobe in der linken Spalte das HAADF-Signal, das die Position des Strahls auf der Probe angibt. Daneben befindet sich das hintergrundkorrigierte EELS-Spektrum im Bereich der Bor-K-Kante und auf der rechten Seite schließt sich das hintergrundkorrigierte EELS-Spektrum im Bereich der Sauerstoff-K-Kante an. Abbildung 4.24 zeigt die gleiche Messung an der Probe mit der Ta-Deckschicht.

Die Probe mit der Ru-Deckschicht (Abbildung 4.23) zeigt, wie zu erwarten, ein klares Sauerstoffsignal im Bereich des $\mathrm{MgO}$. Bor ist weder im $\mathrm{MgO}$ oder am Übergang zum CoFe, noch im kristallisierten CoFe feststellbar. Das Bor scheint also vollständig aus dem c-CoFe herausdiffundiert zu sein. An das CoFe grenzt ein schmaler Bereich an, der im HAADF-Signal als dunkler Kontrast erscheint. In diesem amorphen Bereich ist praktisch das ganze Bor zu finden. In der Ru-Deckschicht selbst kann wiederum kein Bor nachgewiesen werden. 


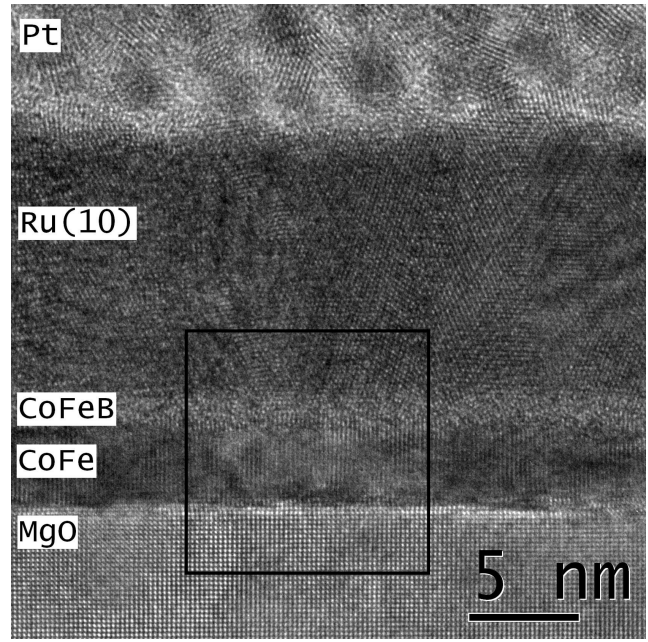

(a)

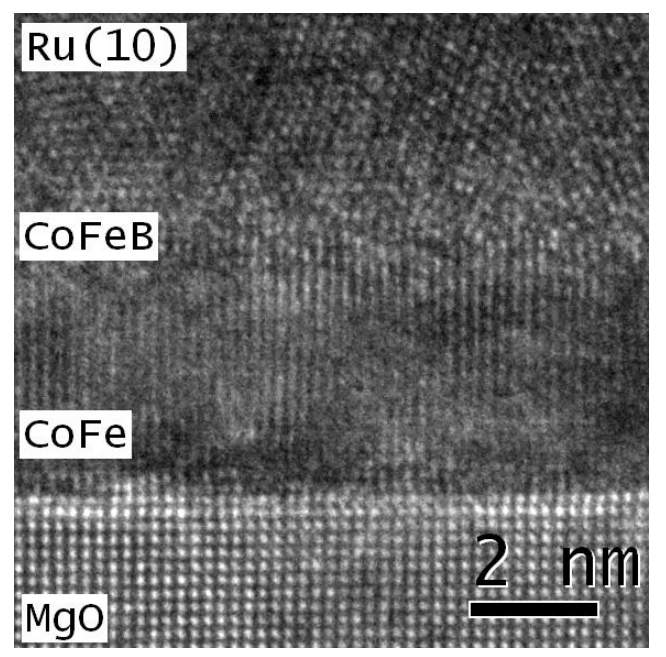

(c)



(b)



(d)

Abbildung 4.22.: TEM-Abbildungen von ausgelagerten Proben mit 5 nm dünner CoFeB-Schicht. Links mit Ru-Deckschicht und rechts mit Ta-Deckschicht. Die RuDeckschichtprobe zeigt eine amorphe Schicht zwischen kristallisiertem CoFe und RuDeckschicht. Die Abbildungen c) und d) sind Ausschnitte von a) und b). [117] 


\begin{abstract}
Abbildung 4.23:
Hintergrundkorrigierte EELS-Messung der RuDeckschichtprobe. Links das zur EELS-Messung gehörende HAADF-Signal. Mitte und rechts das EELSBor-K-Kanten-Signal und das EELS-Sauerstoff- $K$ Kanten-Signal jeweils zum dazugehörigen Ort. Gut sichtbar ist das konzentrierte Bor-Signal in der Zwischenschicht zwischen kristallisiertem CoFe und Ru. Im $\mathrm{MgO}$ oder an dessen Grenzfläche zum CoFe ist dagegen kein B-Signal messbar. [117]
\end{abstract}

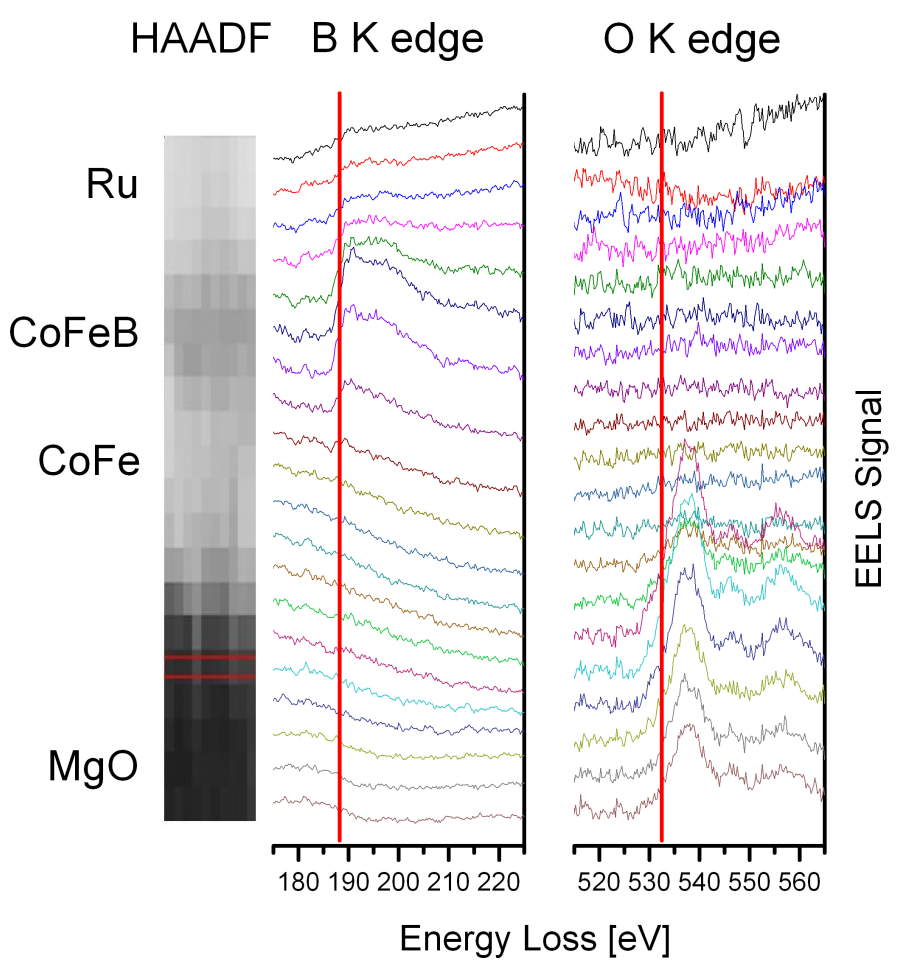

Da in den hellen Zwischenschichten der TEM-Abbildungen mittels EELS Bor festgestellt wurde, erklärt anscheinend das schwach streuende Bor den hellen Kontrast in diesen Schichten.

Die Ta-Deckschichtprobe (Abbildung 4.24) zeigt im $\mathrm{MgO}$ naturgemäß ein Sauerstoffsignal gefolgt von einem Bor-freien Bereich im kristallisierten Co$\mathrm{Fe}$ an. Erst die folgenden Messpunkte weisen ein Bor-Signal auf, das sich bis zum Ende des Messbereiches in das Ta hineinerstreckt. Auch in dieser Probe kann kein Bor im $\mathrm{MgO}$ oder an der Grenzfläche $\mathrm{MgO} / \mathrm{CoFe}$ festgestellt werden.

Aus den TEM-Abbildungen mit der $100 \mathrm{~nm}$ dicken CoFeB-Schicht wird deutlich, dass die Ru-Deckschicht die Kristallisation des CoFe beim Auslagern durch seine polykristalline Struktur beeinflussen kann, wohingegen bei der Ta-Deckschicht ausschließlich eine Kristallisation vom MgO auftritt.

Die amorphe Zwischenschicht der Ru-Deckschichtprobe kann mittels EELSMessungen als borhaltig identifiziert werden. Da in dieser Probe eine dünnere, kristalline CoFe-Schicht als in der Vergleichsprobe mit einer Ta-Deckschicht vorhanden ist, ist davon auszugehen, dass es sich um eine nicht kristallisierte Schicht CoFeB handelt. Die $100 \mathrm{~nm}$ CoFe-Probe mit Ru-Deckschicht zeigt bei gleicher Auslagerungszeit und Temperatur eine 13 nm dicke, kristalline CoFe- 


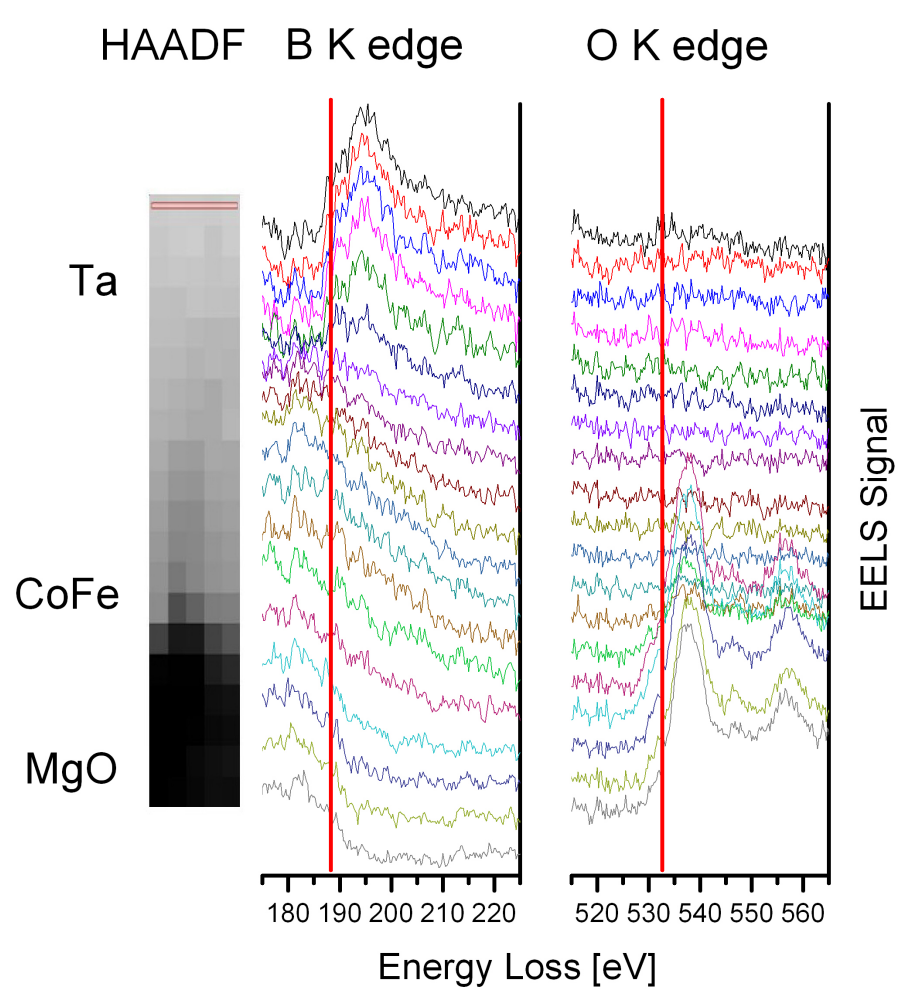

Abbildung 4.24:

Hintergrundkorrigierte

EELS-Messung der TaDeckschichtprobe. Links das zur EELS-Messung gehörende HAADF-Signal. Mitte und rechts das EELSBor-K-Kanten-Signal und das EELS-Sauerstoff-KKanten-Signal jeweils zum dazugehörigen Ort. Das B-Signal erstreckt sich weit in das Ta hinein. Ebenfalls kein $B$ im $M g O$ oder an der Grenzfläche zum $\mathrm{MgO}$. [117]

Schicht zum $\mathrm{MgO}$, was zeigt, dass die Auslagerungsbedingungen ausreichen, um die $5 \mathrm{~nm}$ dicke CoFeB-Schicht zu kristallisieren.

Aus den EELS-Messungen lässt sich folgern, dass das Ru die Bor-Diffusion in die Deckschicht blockiert, da dort kein Bor zu finden ist. Dies würde bedeuten, dass das $\mathrm{CoFeB}$ beim Auslagern vom $\mathrm{MgO}$ aus kristallisiert und sich das Bor im noch nicht kristallisierten $\mathrm{CoFeB}$ anreichert, da die darüberliegende Ru-Deckschicht das Bor nicht aufnimmt. Dadurch verlangsamt sich die Kristallisation des CoFeB beim Auslagern und es bleibt anschließend eine amorphe Zwischenschicht zurück, welche praktisch das gesamte Bor enthält.

Anders sieht es im Fall der Ta-Deckschichtprobe aus. Hier scheint das CoFe vollständig kristallisiert zu sein. Aus den EELS-Messungen lässt sich schließen, dass das Bor beim Auslagern in das Ta hineindiffundiert und das Ta als Senke für das Bor dient. Beiden Proben ist gemein, dass weder Bor noch Boroxid im

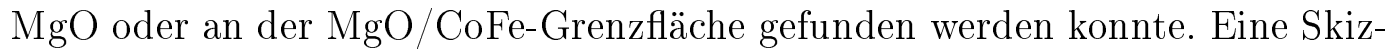
ze, die die Veränderungen während des Auslagerns darstellt, ist in Abbildung 4.25 dargestellt.

Bei den 100 nm dicken CoFeB-Schichten sind in der Ru-Deckschichtprobe 




Abbildung 4.25.: Schematische Darstellung der Probenveränderung während der Auslagerung mit $5 \mathrm{~nm}$ dicker CoFeB-Schicht.

$38 \%$ des CoFeB kristallisiert. Geht man davon aus, dass das Bor weder in das angrenzende Ruthenium noch in das $\mathrm{MgO}$ übergegangen ist, müsste es sich im amorphen $\mathrm{CoFeB}$ angereichert haben. Geht man von einer Ausgangskonzentration von $20 \%$ Bor im CoFeB aus, so müsste sich der Borgehalt in dieser Probe auf $32 \%$ im amorphen $\mathrm{CoFeB}$ angereichert haben. Diese erhöhte Konzentration von Bor könnte erklären, weshalb die vom $\mathrm{MgO}$ aus kristallisierte CoFe-Schicht in der Ru-Deckschichtprobe dünner ausfällt als in der Ta-Deckschichtprobe.

\subsubsection{Einfluss der MgO-Depositionsmethode auf die Bor-Diffusion}

Die in Anschnitt 4.2.1 gewonnenen Erkenntnisse werden in diesem Kapitel auf reale TMR-Systeme erweitert. Außerdem wird der Frage nachgegangen, welchen Einfluss die Herstellungsweise der MgO-Barriere auf die Bor-Verteilung nach dem Auslagern aufweist. Dazu werden TEM-Proben von zwei MTJs präpariert, die beide einen ähnlichen TMR von etwa $200 \%$ aufweisen, aber mit unterschiedlichen MgO-Depositionsmethoden hergestellt wurden. Die Funktionsweise der hier verwendeten Depositionsverfahren ist im Anhang unter Abschnitt B näher erläutert. In der [rf]-Probe wurde die MgO-Barriere per rfSputtern hergestellt und bei der [eb]-Probe mittels Elektronenstrahlverdampfen. Die genaue Zusammensetzung und Schichtfolge ist ebenfalls in Anhang unter Abschnitt B.4 zu finden.

Die HRTEM-Abbildungen 4.26a und 4.26b zeigen einen Querschnitt durch das MTJ mit der MgO-Tunnelbarriere als zentrales Element, umschlossen von 


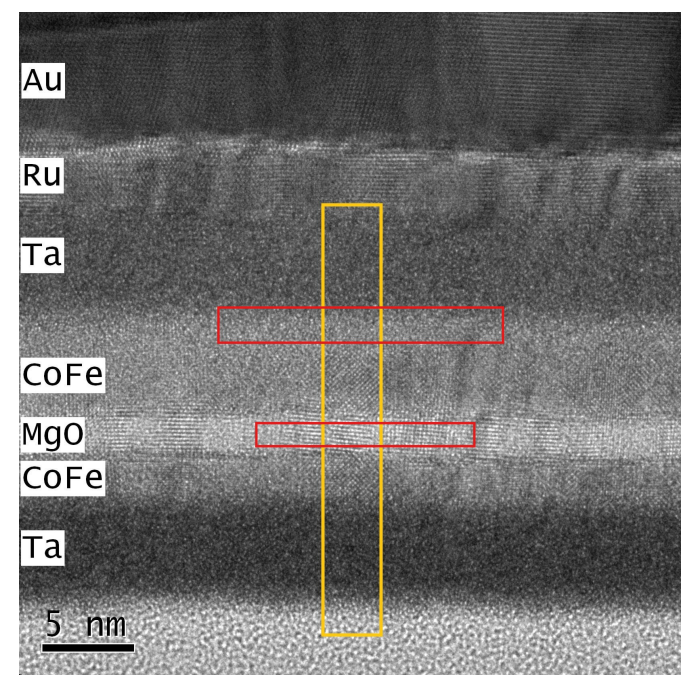

(a)

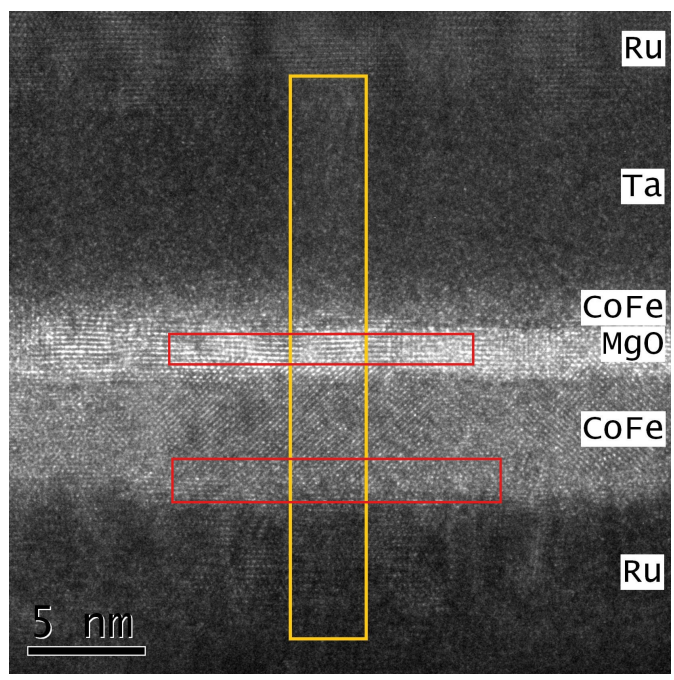

(b)

Abbildung 4.26.: HRTEM-Abbildungen der [eb]-Probe (a) und der [rf]-Probe (b). Beide Proben zeigen eine kristalline MgO-Tunnelbarriere und kristallisierte CoFeElektroden. Die gelben Rechtecke kennzeichnen symbolisch den Bereich für die anschließenden EELS-Messungen. Die rot eingezeichneten Rechtecke kennzeichnen den Bereich für die detaillierten EELS-Messungen an der Bor- und der Sauerstoff-Kante in Abbildung 4.28 .

den CoFeB-Elektroden. Beide MTJs weisen eine gleichmäßig kristalline MgOTunnelbarriere und - durch das Auslagern - kristallisierte Elektroden mit einer scharfen Grenzfläche zum MgO auf. Außerdem kann man in der [rf]-Probe zwischen der unteren CoFe-Elektrode und der darunter liegenden Ru-Schicht eine dünne amorphe Zwischenschicht erkennen, die einen helleren Kontrast aufweist (unterer roter Kasten in Abbildung 4.26b). Ein ähnlich heller Kontrast ist auch in der [eb]-Probe zwischen dem oberen CoFe und der Ta-Schicht sichtbar (oberer roter Kasten in 4.26a).

An diesen Proben werden wie in Abschnitt 4.2.1 EELS-Spektren aufgenommen. Die Aufnahmebedingungen sind identisch mit den dort angegebenen Einstellungen. In der [eb]-Probe befindet sich nur im Bereich der oberen CoFeElektrode zur darüberliegenden Ta-Deckschicht ein Bor-Signal (siehe Pfeil in Abb. 4.27a . Die Messungen am Modellsystem bestätigen, dass Ta als Senke für Bor dient. In der unteren Elektrode und der darunterliegenden Ta-Pufferschicht kann kein Bor nachgewiesen werden. Auch die MgO-Tunnelbarriere zeigt sich hier frei von Bor. 
Das Signal-zu-Rausch-Verhältnis bei der EELS-Messung der [rf]-Probe ist besser als bei der [eb]-Probe. So findet man in der unteren CoFeB-Elektrode deutliche Anzeichen von Bor, ebenso in der oberen Elektrode und in der darüberliegenden Ta-Deckschicht. Auffällig ist in dieser Probe jedoch das BorSignal im Bereich der MgO-Tunnelbarriere, das hier eine andere Form aufweist. Die Feinstruktur dieses Signal unterscheidet sich mit seinen zwei Maxima bei etwa $195 \mathrm{eV}$ und $203 \mathrm{eV}$ deutlich von den einzelnen, zu höheren Energien in die Länge gezogenen Maximum bei $193 \mathrm{eV}$ im CoFeB (siehe Pfeile in 4.27b).

In beiden Proben zeigt sich, dass die in den TEM-Abbildungen sichtbar, hellere Schichten, die an das kristallisierte CoFe angrenzen, borhaltig sind.

Es soll an dieser Stelle erwähnt werden, dass sich die Schichtreihenfolgen in den beiden MTJ leicht unterscheiden. So besteht die an die untere Elektrode angrenzende Schicht im Falle der [rf]-Probe aus Ru, nicht aus Ta wie im Falle der [eb]-Probe. Dieser Umstand sollte jedoch nicht ursächlich für das gefundene Bor in der MgO-Barriere sein, da die [eb]-deponierte MgO-Schicht im Modellsystem in Abschnitt 4.2.1 trotz Ru-Deckschicht völlig frei von Bor ist.

Um diesen Umstand näher zu untersuchen, werden in beiden Proben ELNES-Feinstrukturspektren aufgenommen, die über einen größeren Bereich innerhalb der MgO-Barriere integriert werden. Zum Vergleich wird in beiden Proben ein Spektrum aus einem Bereich aufgenommen, in dem zuvor Bor gefunden wurde, z.B. im hellen Übergangsbereich zwischen CoFe und Ru in der [rf]-Probe und dem Bereich CoFe/Ta in der [eb]-Probe. Diese Bereiche sind in Abbildung 4.26 exemplarisch rot markiert.

In Abbildung 4.28a sind die hintergrundkorrigierten Spektren im Bereich der Bor-K-Kante aus beiden Proben gegenübergestellt. Die [eb]-Probe (rot) zeigt im $\mathrm{MgO}$ kein signifikantes Bor-Signal, im Übergangsbereich CoFe/Ta hingegen ist ein deutlicher Peak erkennbar, der von seiner Form auf metallisches Bor hinweist. In der [rf]-Probe (schwarz) unterscheidet sich das B-K-Signal in der MgO-Tunnelbarriere deutlich vom Spektrum aus dem Bereich $\mathrm{CoFe} / \mathrm{Ru}$. Im MgO weist das ELNES-Spektrum einen signifikanten Peak bei $193 \mathrm{eV}$ auf, der als $\pi^{*}$-Peak des Bors bekannt ist und charakteristisch für $\left[\mathrm{BO}_{3}\right]^{3-}$, also oxidiertes Bor in trigonaler Sauerstoffanordnung ist [31, 85]. Die ELNES-Struktur des Spektrums aus dem Übergangsbereich $\mathrm{CoFe} / \mathrm{Ru}$ weist keinen charakteristischen $\pi^{*}$-Peak auf und zeigt an, dass das Bor hier wie in der [eb]-Probe in metallischer Form vorliegt.

Zusammen mit dem Bor-Signal wird auch im Bereich der O-K-Kante das 




(a)

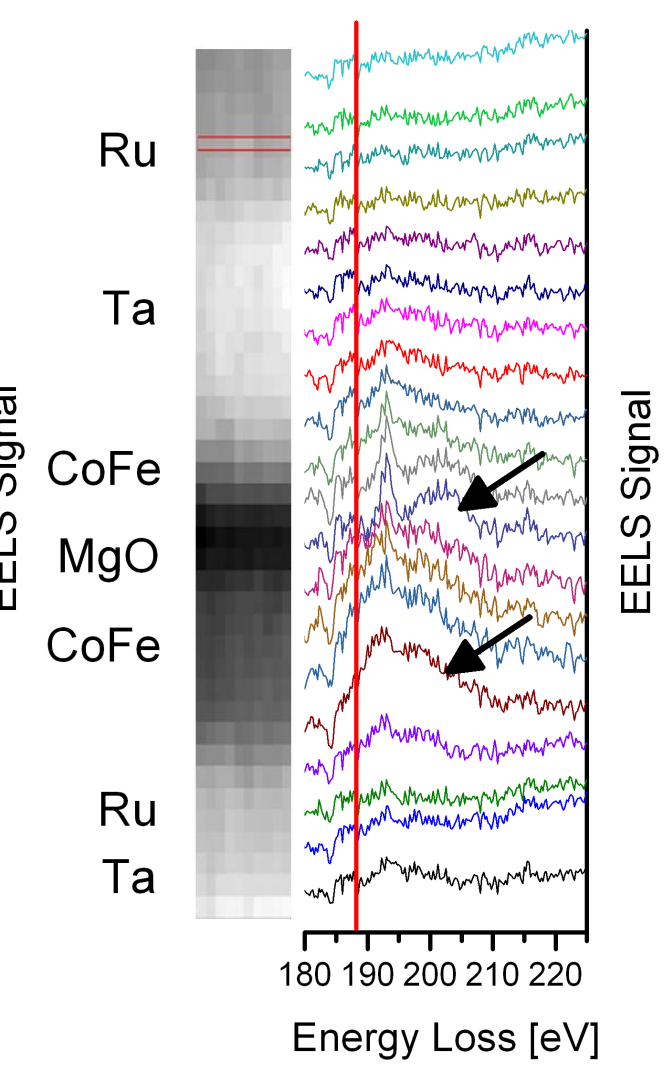

(b)

Abbildung 4.27.: Hintergrundkorrigierte EELS-Spektren der [eb]-Probe (a) und der [rf]-Probe (b) im Bereich der Bor-K-Kante zusammen mit den zum Ort des Spektrums gehörenden HAADF-Signals. [117]

ELNES-Feinstrukturspektrum aufgenommen. In Abbildung 4.28b sind die Spektren aus beiden Proben im Bereich der MgO-Tunnelbarriere dargestellt. Um eine bessere Vergleichbarkeit zu erreichen, werden die Spektren so normiert, dass sie die gleiche Fläche unterhalb des Signals aufweisen. Die ELNESStruktur der [eb]-Probe (rot) weist im direkten Vergleich mit der [rf]-Probe (schwarz) ausgeprägtere Maxima und Minima auf. Da die ELNES sehr sensitiv auf lokale Veränderungen der Kristallstruktur reagiert, deutet das auf eine defektfreiere Struktur in der [eb]-Probe hin [119, 31].

In der [rf]-Probe findet sich darüber hinaus im Bereich von $530-535 \mathrm{eV}$ ein „pre-peak“ (siehe Pfeil in Abb. 4.28b), der in dieser Form auch bei Untersuchungen von Cha et al. [31] gefunden wurde. Aufgrund von „first principle“Berechnungen wird das als Hinweis für das Vorhandensein von "gap states" in 




(a)



(b)

Abbildung 4.28.: ELNES im Energiebereich der B-K-Kante (a) und der O-K-Kante (b): Gut sichtbarer $\pi^{*}$-Peak von $B O_{x}$ bei $193 \mathrm{eV}$ im Bereich des MgO der [rf]-Probe. O-K-Kante der [rf]-Probe weist einen „pre-peak" bei 530 - 535 eV auf (siehe Pfeil), der auf Sauerstoffleerstellen schließen lässt. [117]

der Bandstruktur des $\mathrm{MgO}$ zurückgeführt, die sich hauptsächlich aus Sauerstoffleerstellen ergeben [120].

Um die Frage zu klären, ob die gesamte MgO-Tunnelbarriere in der [rf]-Probe homogen mit Bor durchsetzt ist oder ob nur die Grenzflächen zum CoFe eine Kontamination mit Bor aufweisen, werden aus der Messung in Abbildung $4.26 \mathrm{~b}$ die Bor-Absorptionskantespektren für die obere und untere Grenzfläche und aus der Mitte der MgO-Barriere extrahiert und in Abbildung 4.29 aufgetragen. Zum Vergleich wird das ebenfalls für die B-K-Kante der [eb]-Probe aus Abbildung 4.26a durchgeführt. In der [eb]-Probe ist, wie aus den vorherigen Messungen zu erwarten, weder an den Grenzflächen noch in der MgO-Barriere selbst ein Bor-Signal messbar. In der [rf]-Probe zeigt sich, dass sowohl die obere als auch die untere Grenzfläche zum CoFe identische Peakhöhen und Formen aufweisen wie das Absorptionsspektrum, das aus der Mitte der Barriere stammt. Das bedeutet eine homogene Verteilung des Bors in der gesamten Barriere und nicht wie z.B. bei Karthik et al. nur an den Grenzflächen zum CoFe [25].

\subsubsection{Zusammenfassung der Ergebnisse der Bor-Diffusionsuntersuchungen}

Ziel bei der Herstellung von MTJs ist ein kristalliner Übergang an der Grenzfläche vom $\mathrm{MgO}$ zum CoFe, wozu das Bor beim Auslagern aus der Elektrode 


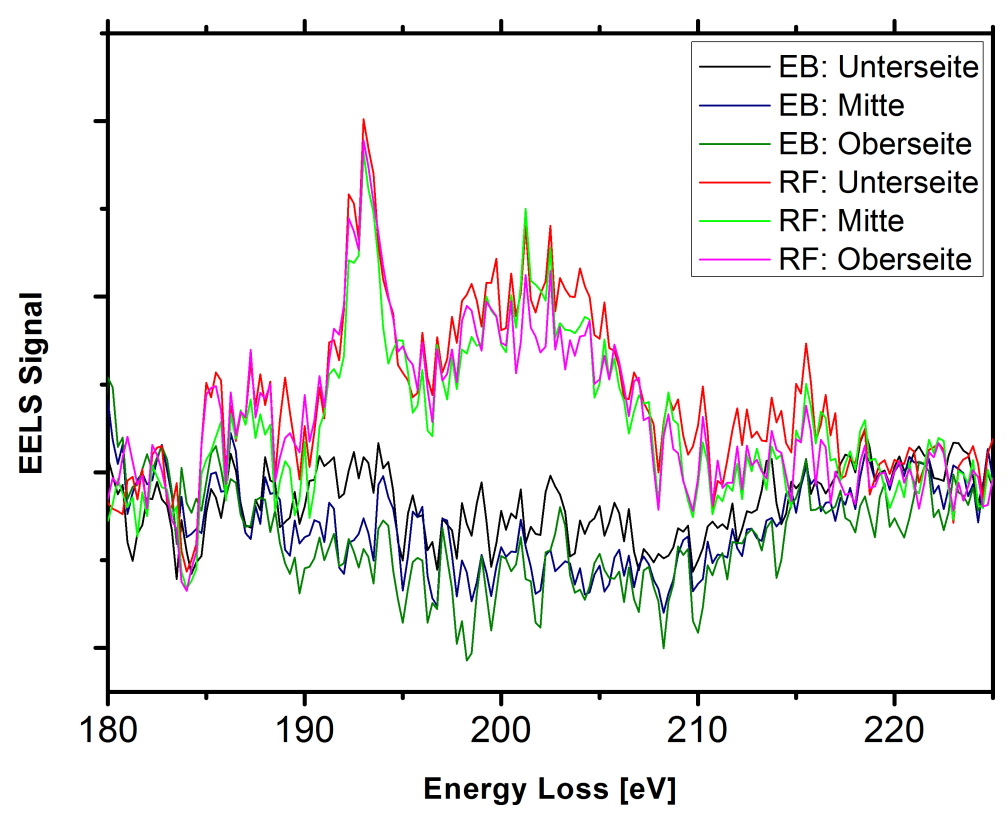

Abbildung 4.29:

Vergleich der $B$ K-Kanten-EELSSpektren für die Positionen an den Grenzflächen und der Mitte der $\mathrm{MgO}$ Barriere für die [eb]und die [rf]-Probe.

herausdiffundieren muss. Die hier vorgestellten Untersuchungen zeigen zwei Möglichkeiten die Bor-Diffusion und die Kristallisation des CoFeB zu beeinflussen. So zeigt sich, dass das Deckschichtmaterial sowohl als Senke, als auch als Diffusionsbarriere für das beim Auslagern herausdiffundierende Bor fungieren kann. Des Weiteren kann die Deckschicht auch eine Kristallisation des CoFeB von der Deckschicht aus initiieren.

So zeigte sich an Ruthenium-Deckschichten nach dem Auslagern eine Schicht kristallisiertes CoFe, das in der Ta-Deckschichtprobe mit der dicken CoFeBSchicht nicht auftritt. Ebenso ist bei dünnen CoFeB-Schichten eine dünne amorphe CoFeB-Schicht zwischen dem kristallinen CoFe und der Ru-Deckschicht übriggeblieben. Ursächlich hierfür könnte die Blockierung der Bordiffusion des Ru sein: Die Kristallisation des $\mathrm{CoFeB}$ startet am MgO. Aus dieser kristallinen CoFe-Schicht diffundiert das Bor heraus und reichert sich in der verbliebenen a-CoFeB-Schicht an. EELS-Messungen zeigen, dass die RuDeckschicht eine Diffusionsbarriere für das Bor darstellt; somit kann das Bor nicht in dieses hineindiffundieren. Aufgrund der hohen Borkonzentration kann das a-CoFeB auch nicht mehr auskristallisieren und die Kristallisation kommt zum Erliegen. Es bleibt eine dünne amorphe CoFeB-Zwischenschicht übrig. Bei den dicken CoFeB-Schichten kann das durch die Kristallisation verdrängte Bor bis zu einem bestimmten Grad von der dicken CoFeB-Schicht zwischen Ru und $\mathrm{MgO}$ aufgenommen werden. Bei den dünnen Schichten führt das dazu, dass eine amorphe Zwischenschicht nicht mehr auskristallisieren kann. Im Vergleich zu den dicken CoFeB-Schichten kommt es somit nicht zu einer Kristallisation 
von der Deckschicht aus.

Im Falle einer Ta-Deckschicht zeigen EELS-Messungen, dass das Bor in dieses hineindiffundiert und es somit eine Senke für das Bor darstellt. Damit kann das CoFeB hier vollständig kristallisieren.

Daraus wird deutlich, dass Ta als Deckschicht ein deutlich besser geeignetes Material darstellt als Ru und deshalb auch höhere TMR-Werte aufweist (s. Abb. 2.8.

Wie sich in den weiteren hier vorgestellten Untersuchungen deutlich zeigt, ist auch die Herstellungsweise der MgO-Tunnelbarriere entscheidend. Während die eb-deponierte Probe keine Kontamination der Barriere mit $\mathrm{B}$ oder $\mathrm{BO}_{\mathrm{x}}$ aufweist, zeigt die rf-gesputterte Probe in den EELS-Spektren ein deutliches $\mathrm{BO}_{\mathrm{x}}$-Signal. Die Ursache dieser $\mathrm{BO}_{\mathrm{x}}$-Kontamination kann hier nicht geklärt werden. Es ist davon auszugehen, dass dies durch eine substöchiometrische Zusammensetzung der MgO-Tunnelbarriere durch das rf-Sputterverfahren hervorgerufen wird. Ob dies ein Sauerstoffdefizit oder ein Sauerstoffüberschuss ist, kann in diesen dünnen MgO-Schichten mittels EELS nicht mit hinreichender Genauigkeit gemessen werden.

In Berechnungen von Velev et al. wurde gezeigt, dass Sauerstoffleerstellen in der MgO-Barriere ebenfalls den TMR beeinflussen. So zeigten die Berechnungen, dass der TMR von $1800 \%$ im Fall einer idealen MgO-Barriere durch Sauerstoffleerstellen auf unter $800 \%$ reduziert wird [120]. Beide hier untersuchten MTJ weisen einen TMR von $200 \%$ auf, was drauf hindeutet, dass weder die erhöhte Borkonzentration in der MgO-Barriere noch die beobachteten „gap

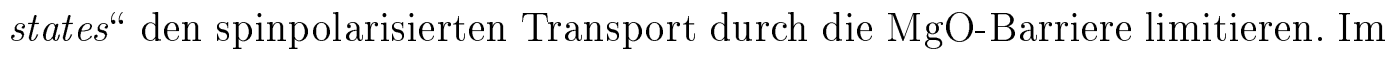
Gegensatz zu Untersuchungen, in denen das Bor nur an der Grenzfläche zwischen $\mathrm{MgO}$ und $\mathrm{CoFe}$ gefunden wurde [32, 25, 30], wurde hier eine gleichmäßige Borkonzentration über die ganze $\mathrm{MgO}$-Barriere hinweg beobachtet, wie es auch bei Harnchana et al. [121] der Fall war. Burton et al. [24] konnten zeigen, dass eine hohe Borkonzentration an den Grenzflächen der MgO-Barriere den spinpolarisierten Transport durch die MgO-Barriere massiv beeinträchtigt, was die besondere Bedeutung der $\mathrm{MgO} / \mathrm{CoFe}(\mathrm{B})$-Grenzflächen erneut unterstreicht. 


\subsection{Zusammenbruch elektrisch gestresster MTJs}

Für die praktische Anwendung ist nicht nur das Erzielen hoher TMR-Werte notwendig, wichtig ist auch eine lange, zuverlässige Lebensdauer der MTJs. In diesem Abschnitt geht es um eine Fortsetzung der Untersuchungen von Eilers [45] an überspannungszerstörten MTJs [34, 122]. Diese Untersuchungen werden auf Proben ausgedehnt, die nur durch kurz Spannungsstöße elektrisch gestresst wurden. Schäfers et al. stellten dabei fest, dass es bei dem dielektrischen Zusammenbruch von MTJs mit ultradünner Barriere zu einer großflächigen Elektromigration im Element kommt [122]. Abbildung 4.30 zeigt zwei TEMQuerschnitte durch zwei strukturierte Elemente, welche mit farblich codierten EDX-Karten überlagert wurden. Die dünne MgO-Tunnelbarriere ist in diesen Abbildungen nicht zu erkennen, sie befindet sich von den CoFeB-Elektroden umschlossen im rot dargestellten Bereich in Abbildung 4.30a.



(a)

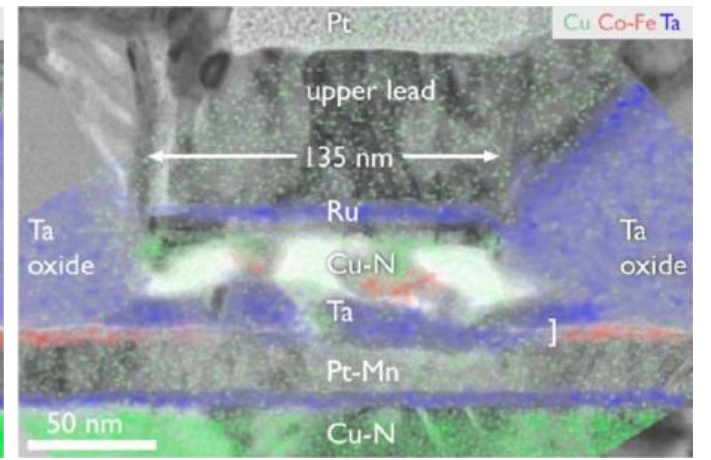

(b)

Abbildung 4.30.: Die TEM-Abbildung zeigt ein intaktes (a) und die großflächige Zerstörung in einem überlasteten (b) MTJ mit farbig überlagerter EDX-Messung von $\mathrm{Cu}$, Co-Fe und Ta. Aus [122].

Während Abbildung 4.30a ein vollständig intaktes Element mit 86\% TMR zeigt, weist das Element in Abbildung 4.30b keinen TMR mehr auf. Die farbige Elementverteilungskarte macht deutlich, dass es zu einer starken Vermischung des Materials gekommen ist. Cobalt und Eisen (rot dargestellt) aus den Elektroden befinden sich um mehrere zehn nm versetzt oberhalb ihrer vorherigen Position wieder, wogegen das Kupfer aus dem Bereich über der Barriere in die entgegengesetzte Richtung versetzt wurde. In [45, 122] wurde gefolgert, dass es aufgrund der hohen Stromdichten beim Durchbruch durch die Barriere zu Elektromigration kam. Cobalt und Eisen werden vom elektri- 
schen Feld getrieben und in einem als direct force bezeichneten Mechanismus nach oben versetzt. Das Kupfer wird in einem als wind force bezeichneten Mechanismus in Richtung des Elektronenstroms mitgerissen.

Ebenso wurden Durchbrüche, auch pinholes genannt, in der MgO-Barriere elektrisch überlasteter MTJs gefunden. Abbildung 4.31b zeigt einen solchen Durchbruch, wohingegen 4.31a einen Ausschnitt der Tunnelbarriere eines intakten Elements zeigt.



(a)

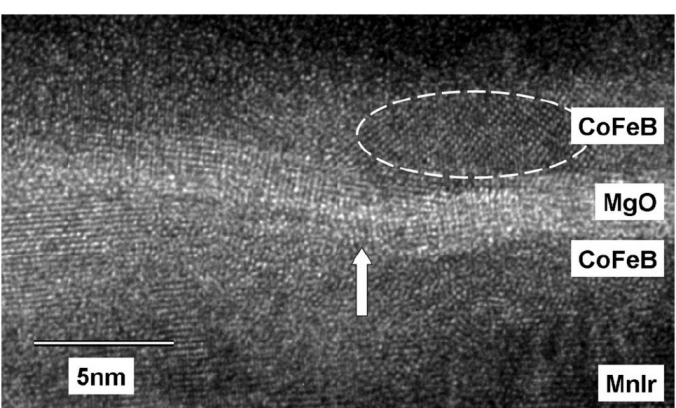

(b)

Abbildung 4.31.: HRTEM-Abbildungen der MgO-Tunnelbarriere eines intakten (a) und defekten (b) MTJs zwischen zwei CoFeB-Elektroden. Das zerstörte Element zeigt einen Bruch in der Tunnelbarriere (Pfeil) und einen kristallisierten CoFe-Bereich über dem Durchbruch. Aus [34].

Oberhalb des mit einem weißen Pfeil gekennzeichneten Durchbruchs in Abbildung $4.31 \mathrm{~b}$ ist ein Bereich kristallinen $\mathrm{CoFe} z u$ sehen. Dieser kristalline Bereich ist Folge lokalen „annealings“. Das ,annealing“ entsteht, weil sich der Flächenwiderstand verringert und infolgedessen ein erhöhter Strom durch den Durchbruch fließt.

Im Folgenden werden MTJs untersucht, die wie die oben beschriebenen von Andy Thomas' Arbeitsgruppe Thin Films and Physics of Nanostructures an der Universität Bielefeld strukturiert und elektrisch vermessen wurden. Die Herstellungsbedingungen und Schichtreihenfolgen sind im Anhang unter Abschnitt B.5 zu finden. Bei allen Elementen wird vor und nach der elektrischen Belastung der TMR bestimmt. Die elektrische Belastung zeichnet sich durch einen Spannungspuls aus, dessen Amplitude linear von $0 \mathrm{~V}$ bis $3 \mathrm{~V}$ ansteigt. Die Pulsdauer beträgt 50 ns oder $1 \mu$ s. Die untersuchten elliptischen Tunnelelemente weisen dabei eine Größe von $380 \mathrm{~nm}$ x $150 \mathrm{~nm}$ auf und werden mittels FIB-Zielpräparation für die TEM-Untersuchungen präpariert. 

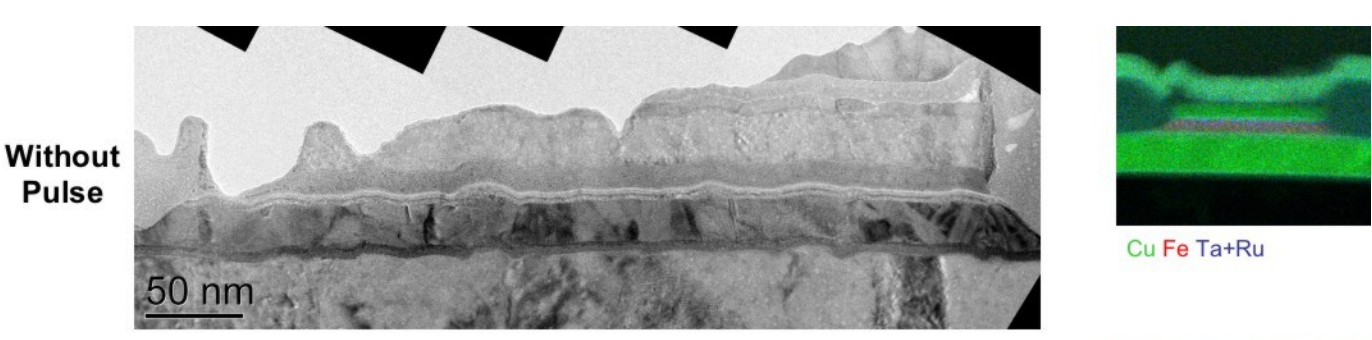

Cu Fe Ta+Ru


$\mathrm{Cu} \mathrm{Co}+\mathrm{Fe} \mathrm{Ta}+\mathrm{Ru}$
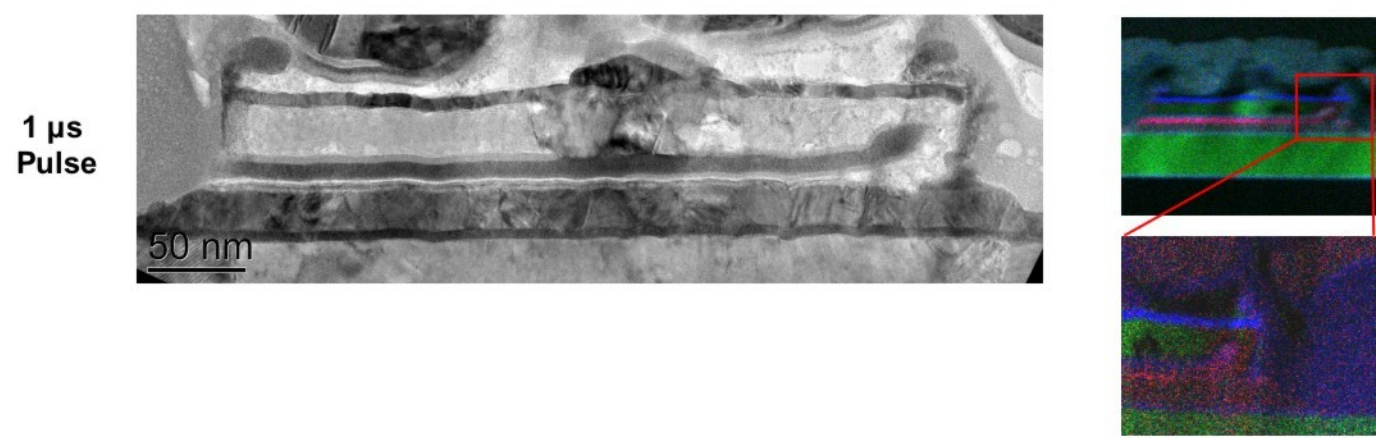

$\mathrm{Cu} \mathrm{Co}+\mathrm{Fe} \mathrm{Ta}+\mathrm{Ru}$

Abbildung 4.32.: Links: TEM-Abbildungen strukturierter MTJs für unterschiedlich belastete Elemente. Rechts: dazugehörige farbcodierte EDX-Messung für Cu, Co-Fe und Ta-Ru. Die beiden oberen Abbildungen zeigen ein unbelastetes, intaktes MTJ. Mitte: ein Element, das 50 ns lang gestresst wurde. Unten: ein MTJ, das 1 ps lang bis zum dielektrischen Zusammenbruch belastet wurde.

Abbildung 4.32 zeigt Querschnitte von drei MTJs. Auf der rechten Seite sind die dazugehörenden farbigen EDX-Elementverteilungskarten dargestellt, in der $\mathrm{Cu}$ grün, $\mathrm{Co}-\mathrm{Fe}$ rot und $\mathrm{Ta}$ und $\mathrm{Ru}$ blau eingefärbt sind. Das obere MTJ zeigt ein intaktes Element, das einen TMR von $\sim 100 \%$ aufweist. Das mittlere MTJ wurde mit einem Spannungspuls von $50 \mathrm{~ns}$ belastet und wies vor der Belastung einen TMR von $91 \%$ und danach einen Rest-TMR von $2,3 \%$ auf. Das untere Element wurde $1 \mu$ s lang belastet und weist nach der Belastung keinen TMR mehr auf; ist also vollständig zerstört. Vorher wurden 
noch $102 \%$ TMR an dieser Probe gemessen.

Die TEM- und EDX-Abbildungen aller Elemente zeigen keine großflächigen Veränderungen, wie es in Abbildung 4.30 der Fall war. Die MgO-Tunnelbarrieren, in den TEM-Abbildungen als dünne helle Schicht sichtbar, weisen leichte Unebenheiten auf, sind aber auch im intakten MTJ zu finden und resultieren aus Unebenheiten der unteren Schichten oder des Substrats. Beim unteren, mit $1 \mu \mathrm{s}$ belasteten MTJ, ist am rechten Rand des Elements eine Veränderung zu erkennen, die ein Aufbrechen der Ta-Schicht über der oberen Elektrode vermuten lässt. Die EDX-Elementverteilungskarte auf der rechten Seite bestätigt die Vermutung. Die Elektroden mit der dazwischen liegenden Tunnelbarriere, hier rot dargestellt, weisen, bis auf den rechten Teil des MTJ, einen geraden Verlauf auf. Im rechten Bereich, der im Ausschnitt nochmals vergrößert dargestellt ist, ist zu erkennen, dass das CoFe der Elektroden wie in Abb 4.30b nach oben versetzt worden ist. Hier ist es, vermutlich durch Ungenauigkeiten bei der Strukturierung, zu einer Schwachstelle gekommen, die bei kurzzeitiger Belastung zu einem Durchbruch führt. Man kann erkennen, dass das CoFe, wie in den Untersuchungen von Schäfer et al. [122, nach der Belastung oberhalb seiner ursprünglichen Position zu finden ist. Sie folgerten, dass das CoFe im elektrischen Feld nach oben mitgerissen wurde und damit das MTJ zerstörte. Somit ist die hier vorgefundene Situation mit der bei Schäfer et al. kompatibel.

Bei den mit Pulsen von 50 ns belasteten mittleren MTJs sind weder in der TEM-Abbildung noch in der EDX-Elementverteilungskarte Aufbrüche der Barriere oder Vermischungen zu erkennen. Hier ist also der Stromfluss nicht hoch genug, um das Element vollständig durch Elektromigration zu zerstören.

Die HRTEM-Abbildung 4.33 eines $50 \mathrm{~ns}$ lang belasteten MTJs zeigt, wie schon bei den vollständig zerstörten MTJs, einen Bruch der Barriere. Jedoch ist in diesem Fall keine Kristallisation der oberen Elektrode aufgrund von lokalem „,annealing“ aufgetreten. Die Stromdichten nach dem Bruch der Barriere sind folglich nicht so groß, um diese zu bewirken. Das hier gezeigte Element zeigt vor der gezielten Belastung einen TMR von $96 \%$ und trotz Bruch der MgO-Barriere noch $11 \%$ TMR nach der Belastung.

Aus diesen Ergebnissen ergeben sich zusammen mit den vorherigen Erkenntnissen mehrere Stadien für den Zusammenbruch von MTJs unter Belastung.

- Bei kurzen Belastungen erfolgt ein Bruch der isolierenden Barriere, was einhergeht mit einer Reduktion des Flächenwiderstandes und des TMR. Hierbei ist anzunehmen, dass der Bruch vornehmlich an Schwachstellen des MTJ, also Fehlern bei der Strukturierung, Maskenfehlern, etc. 




Abbildung 4.33.: Die HRTEM-Abbildung eines pinholes (Pfeil) in der isolierenden Tunnelbarriere eines MTJ mit $11 \%$ Rest-TMR zeigt keine Kristallisation aufgrund lokalen „annealings “ an der oberen Elektrode (Kreis).

oder eventuell auch an Korngrenzen innerhalb der texturierten $\mathrm{MgO}$ Barriere geschieht. Untersuchungen von Komagaki et al. [33] zeigen, dass es beim Auslagern hauptsächlich zu einer Diffusion von Bor entlang von Korngrenzen ins $\mathrm{MgO}$ kommt, welche den Flächenwiderstand verringern. Diese lokale Änderung des Barrierenwiderstandes durch das Bor an den Korngrenzen kann die Bildung von pinholes bei Überlastung begünstigen. Eine Verringerung der Texturierung und eine damit einhergehende Verringerung der Korngrenzen in der Barriere würde auch die Dichte an Diffusionsmöglichkeiten von Bor in die Barriere reduzieren und somit die Langlebigkeit dieser MTJs erhöhen. Um die Texturierung von MgOBarrieren quantifizieren zu können, wurde von Peretzki [123] hierfür eine Methode entwickelt, um diese in HRTEM-Abbildungen zu bestimmen.

- Beim Bruch der isolierenden Barriere kommt es zu einer Reduktion des Widerstandes, was zu einem weiteren Anstieg der Stromdichte führt. Bei anhaltender Belastung kommt es durch den erhöhten Stromfluss zu einem lokalen ,annealing“" der oberen Elektrode [34].

- Durch Elektromigration kommt es in überlasteten MTJs zu einer vollständigen Zerstörung. Laut Schäfers et al. werden Cobalt und Eisen 
durch das elektrische Feld aus ihrer ursprünglichen Position heraus getrieben, während Kupfer in Richtung des Elektronenstroms mitgerissen wird; das führt zu einer vollständigen Zerstörung des MTJs [122]. 
$\Gamma_{\text {Kapitel }}$

Zusammenfassende Diskussion und Ausblick

Nach aktuellem Stand der Forschung sollen bereits wenige Monolagen kristallinen Eisens an der Grenzfläche zur MgO-Tunnelbarriere ausreichen, um mit vollständig kristallinen Elektroden vergleichbare TMR-Werte zu erzielen [11]. In Systemen mit amorphen CoFeB-Elektroden erreicht man eine Kristallisation an der Grenzfläche durch einen Auslagerungsschritt bei Temperaturen über $300{ }^{\circ} \mathrm{C}$ [23, 46], bei dem das Bor aus den Elektroden herausdiffundiert. Für das Bor ist es jedoch energetisch günstiger an der Grenzfläche zu bleiben, anstatt im kristallisierten CoFe, was eine drastische Reduktion des TMR zur Folge hat [24]. Die Kristallisation und die Diffusion von Bor sind bislang schwer zu kontrollierende Prozesse. Diese für den TMR sehr wichtigen strukturellen und chemischen Eigenschaften der Grenzfläche wurden in dieser Arbeit mittels quantitativer, hochauflösender und analytischer Elektronenmikrokopie analysiert.

Um die vom kristallinen $\mathrm{MgO}$ aus induzierte kristalline Ordnung an der Grenzfläche zu bestimmen und eine minimale Reichweite hierfür angeben zu können, wurden Untersuchungen an einem Modellsystem durchgeführt. Dieses System bestand aus einer auf ein MgO-Substrat aufgesputterten und nicht ausgelagerten CoFeB-Schicht.

Zur Untersuchung wurde das von Borgardt, Plikat, Thiel, Schröter und Seibt [13, 14, 21, 51, 22, 52, 53. entwickelte und genutzte APP-Verfahren zur Untersuchung amorph/kristalliner Grenzflächen auf dieses System angepasst (s. Abschnitt 3.3) und erweitert.

Die Untersuchung zeigte, dass acht Parameter ausreichen, um die Vertei- 
lungsfunktion für dieses System zu beschreiben, da es unter Verwendung von zehn Parametern zu keiner signifikant besseren Beschreibung der Grenzfläche kam. Einen großen Einfluss auf die Kontraste haben jedoch Inhomogenitäten entlang der Grenzfläche.

Bei Plikat und Thiel [21, 22, 52, 53] wurden für die Untersuchungen spezielle Substrate verwendet, die eine weitgehend atomar glatte Oberfläche vor der Deposition gewährleisteten und somit keine Berücksichtigung von Stufen an der Grenzfläche in der Simulation der Abbildungen benötigten. Auf den in dieser Arbeit verwendeten MgO-Substraten wurde vor dem CoFeB eine $6 \mathrm{~nm}$ dicke MgO-Schicht aufgebracht, um glattere Oberflächen zu erhalten. Trotz dieser Maßnahme zeigte die hier untersuchte Probe eine im Vergleich zu Plikat und Thiel deutlich größere Anzahl an Stufen an der Grenzfläche auf.

Es konnte gezeigt werden, dass sich in dem hier untersuchten Probenbereich eine Stufe entlang der Strahlrichtung befindet. Dabei zeigte die Anpassung, dass die MgO-Schicht, die in Strahlrichtung oben liegt, eine Monolage weiter in das $\mathrm{CoFeB}$ hineinreicht als die darunterliegende $\mathrm{MgO}-\mathrm{Schicht}$. Dabei sind beide Schichten jeweils 5,5 nm dick. Diese Arbeit beschränkt sich an das Anpassen einer möglichen Stufe in der Lamelle. Da in den Querschnitten etwa alle 4-6 nm eine sichtbare Stufe auftritt ist diese Anzahl auch wahrscheinlich. Darüber hinaus würde eine größere Anzahl an Stufen in Strahlrichtung die Anzahl an möglichen Realisierungen schnell ansteigen lassen. Eine solche iterative Anpassung ist aber aufgrund der immer weiter ansteigenden Computerleistung in folgenden Arbeiten denkbar.

Die iterative Anpassung an verschiedene Stufenpositionen zeigt, dass im Bereich $n_{\text {Pos } 2} / n_{\text {Pos } 3}=11 / 15-14 / 12$ die angepassten Parameter und damit auch die zweidimensionalen Verteilungsfunktionen keine signifikanten Änderungen aufweisen.

Der Abstand zwischen dem letzten Sauerstoffatom im MgO und dem ersten $\mathrm{Co} / \mathrm{Fe}-$ Atom wurde unter Berücksichtigung der Stufe an der Grenzfläche mit $d_{a 1}=0,212 \mathrm{~nm}$ bestimmt. Dieser Abstand ist $15 \%$ größer als der unter Abschnitt 4.1.1.1 bestimmte Abstand an der c-MgO/c-CoFe-Grenzfläche, also an einem ausgelagerten System.

Berechnungen von Burton et al. [24] an kristallisierten CoFeB zeigten einen Fe-O Abstand von $d_{F e-O}=0,223 \mathrm{~nm}$. Der hier gefundene Abstand ist somit $5 \%$ kleiner. Jedoch ist der ebenfalls berechnete Co-O-Abstand mit $d_{C o-O}=$ 0,216 nm nur $2 \%$ größer als der hier gemessene.

Berechnungen von Butler et al. 7] an Fe-MgO-Systemen ergaben einen im Vergleich zum CoFeB-MgO-System reduzierten Fe-O-Abstand von $d_{F e-O}=$ 0, $2169 \mathrm{~nm}$. Dieser stimmt bis auf $2 \%$ mit dem hier gemessenen überein.

Trotz der erschwerten Interpretation der einzelnen Parameter wird durch 
das Modell die Grenzfläche zwischen $\mathrm{MgO}$ und dem metallischen CoFeB gut beschrieben. So konnte die Weite des Einflussbereiches der kristallinen Ordnung auf das amorphe $\mathrm{CoFeB}$ in Grenzflächennähe mit $\approx 0,7 \mathrm{~nm}$ ermittelt werden. Dieser Einflussbereich erstreckt sich somit über 3-4 Atomlagen CoFe, wobei aus dem projizierten Potential gut zu erkennen ist, dass insbesondere die erste Atomlage stark lokalisiert ist.

Im Vergleich mit der Weite des Bereiches mit modifizierter amorpher Struktur in kovalent gebundenen Materialien wie dem System c-Si/a-Ge oder c$\mathrm{Si} / \mathrm{a}-\mathrm{Si}$ bei Thiel [53, 21] von 1,4 nm, ist die hier gefundene Weite in einem metallischem System nur halb so groß. Die Ursache hierfür liegt in den stark gerichteten, kovalenten Bindungen, die in diesem metallischen System so nicht gegeben sind.

Mit 3-4 vollständig kristallinen Monolagen Fe an der Grenzfläche sollte laut Heiliger et al. [11] ein TMR erzielt werden können, welcher mit ausgedehnten kristallinen Elektroden vergleichbar ist. Von 3-4 vollständig kristallinen Lagen CoFe kann in dem hier gefundenen System jedoch nicht gesprochen werden. Einzig die erste Monolage CoFe zeigt eine mit vollständig kristallinem Material vergleichbare Lokalisierung. Die kann zwar zu erhöhten TMR-Werten in Systemen mit weitgehend amorphen CoFeB-Elektroden führen, wie z.B. bei Djayaprawira et al. [10], aber nicht an vollständig kristalline Elektroden heranreichen wie z.B. bei Ikeda [4].

Die Berechnungen von Burton et al. zur CoFeB-MgO-Grenzfläche zeigte ebenfalls, dass es für Bor energetisch günstiger ist, an der Grenzfläche zu verbleiben als im kristallisierten CoFe. Eine Segregation von Bor an der Grenzfläche beim Auslagern würde jedoch den TMR negativ beeinflussen [24]. Das Auslagern der Elemente ist jedoch eine elementare Voraussetzung, damit das Bor herausdiffundiert und das CoFeB kristallisieren kann [23, 2, 46]. Um eine gute Kopplung der $\Delta_{1}$-Zustände der Tunnelbarriere mit denen der Elektroden zu erhalten, ist eine Kristallisation der Elektroden von der kristallinen MgO-Tunnelbarriere aus in einer bcc(001)-Struktur notwendig [2]. Eine Kristallisation in einer anderen Kristallstruktur oder Orientierung wäre für das kohärente Tunneln äußerst schädlich. Eine derartige Kristallisation beim Auslagern könnte von der Deckschicht aus erfolgen. Die Deckschicht des CoFeB, die dem $\mathrm{MgO}$ gegenüberliegt, beeinflusst sowohl die Kristallisation als auch die Bor-Diffusion, da das aus der Elektrode heraustretende Bor nur in zwei Richtungen diffundieren kann: in Richtung des $\mathrm{MgO}$ oder zur Deckschicht. Im besten Fall sollte die Deckschicht beim Auslagern keine Kristallisationsvorlage für das CoFeB bilden und das herausdiffundierende Bor aufnehmen.

Für die in dieser Arbeit durchgeführten Untersuchungen des Kristallisati- 
onsverhaltens und der Bordiffusion wurden zwei verschiedene Deckschichten, Tantal und Ruthenium, in Modellsystemen genutzt. Die MTJ mit diesen beiden Materialien weisen tendenziell die höchsten TMR auf und sind deshalb auch die gebräuchlichsten [29]. Tantal weist dabei leicht höhere TMR auf als Ruthenium.

Diese Deckschichten wurden sowohl auf $5 \mathrm{~nm}$ als auch auf $100 \mathrm{~nm}$ dicke CoFeB-Schichten gewachsen, denen ein einkristallines MgO-Substrat zugrunde lag. Alle Modellsystemproben konnten bestätigen, dass das CoFeB beim Auslagern am $\mathrm{MgO}$ kristallisiert. In den Proben mit nanokristalliner Ta-Deckschicht fand eine CoFe-Kristallisation einzig vom $\mathrm{MgO}$ aus statt. Die $5 \mathrm{~nm}$ dicke CoFeB-Schicht war vollständig durchkristallisiert. EELS-Messungen zeigten, dass das Bor beim Auslagern aus dem CoFeB in das Ta diffundiert ist. So konnten hier die Untersuchungen von Karthik et al. [25, 28] bestätigt werden, dass das Ta die Kristallisation des CoFeB nicht beeinflusst. Da Bor im Ta mittels EELS gemessen wurde und auch keine amorphe Zwischenschicht wie bei Miyajima et al. [26] gefunden wurde, kann hier seine Folgerung, dass das Ta die Bordiffusion blockiert, nicht bestätigt werden.

Einzig bei der $100 \mathrm{~nm}$ dicken CoFeB-Probe mit Ru-Deckschicht trat auch eine Kristallisation des $\mathrm{CoFeB}$ an der polykristallinen hcp-Ru-Schicht auf. In der $\mathrm{Ru}$-Deckschichtprobe mit $5 \mathrm{~nm}$ dicker CoFeB-Schicht zeigte sich eine amorphe Zwischenschicht zwischen kristallinem CoFe und Ru. EELS-Messungen konnten bestätigen, dass diese Zwischenschicht eine erhöhte Borkonzentration aufwies, das Ru jedoch kein Bor enthielt. Durch die Blockierung der Bordiffusion in das $\mathrm{Ru}$ hinein, reichert sich das Bor während des Auslagerns im verbleibenden $\mathrm{CoFeB}$ an und behindert die weitere Kristallisation. In der dickeren CoFeB-Schicht-Probe mit Ru-Deckschicht konnte das Bor von der verbleibenden amorphen-CoFeB Schicht aufgenommen werden und ermöglichte so auch eine Kristallisation von der Deckschicht aus. Die am MgO kristallisierte CoFe-Schicht war jedoch dünner als im Falle der Ta-Deckschicht-Probe. Um eine wirklich langsamere Kristallisation des CoFeB unter Verwendung von $\mathrm{Ru}-$ Deckschichten nachzuweisen wären in-situ-Auslagerungsexperimente im TEM denkbar um den Verlauf der Kristallisation während der Auslagerung und mittels EFTEM evtl. den Verlauf der Boranreicherung zu untersuchen.

Lu et al. 27] fanden in ihren Proben mit Ru-Deckschicht Bor im MgO. Ursächlich sollte hierfür eine Kristallisation des $\mathrm{CoFeB}$ vom Ru aus sein. In den in dieser Arbeit untersuchten Proben konnte dagegen Bor weder im $\mathrm{MgO}$ noch an der $\mathrm{MgO} / \mathrm{CoFeB}-$ Grenzfläche noch im kristallisierten $\mathrm{CoFe}$ gemessen werden. Somit scheint die Deckschicht für die Kontamination der MgO-Barriere mit Bor nicht der alleinige Grund hierfür zu sein. 
Die bisher diskutierten strukturellen als auch die chemischen Untersuchungen wurden an vereinfachten Modellsystemen durchgeführt. In funktionsfähigen MTJs wächst das $\mathrm{MgO}$ auf einer amorphen CoFeB-Schicht auf, was zu einem texturierten Wachstum der Tunnelbarriere führt [2]. Diese Texturierung könnte im Zusammenspiel mit einer Bor-Segregation an den Korngrenzen die Ergebnisse von Lu et al. [27] erklären, ebenso könnte diese Segregation zu einer Degradation der MTJs unter elektrischer Belastung führen [33].

Dieser Zusammenbruch von MTJs wurde bei Thomas, Schäfers und Eilers et al. [34, 122, 45] an elektrisch überlasteten und völlig zerstörten Elementen untersucht und in dieser Arbeit auf Tunnelelemente ausgedehnt, die nur einer kurzzeitigen elektrischen Belastung ausgesetzt waren. Diese elektrische Belastung zeichnete sich durch einen Spannungspuls von 50 ns bzw. $1 \mu$ s bei einem linearen Anstieg der Amplitude von 0 bis $3 \mathrm{~V}$ aus. Der Vergleich des TMR vor und nach der elektrischen Belastung der Elemente zeigte eine durch den Prozess starke Degradation des TMR-Wertes. Dieser war bei den länger gestressten MTJs größer als bei den nur 50 ns gestressten Elementen.

Aus den HRTEM-Abbildungen und den EDX-Messungen konnten mögliche Stadien des Zusammenbruchs von MTJs unter elektrischer Belastung identifiziert werden: So erfolgt bei kurzer Belastung der MTJs ein Bruch der isolierenden Barriere, was eine Reduktion des TMR und des Flächenwiderstandes zur Folge hat. Durch den beim Bruch der Barriere reduzierten Flächenwiderstand kommt es zu einem weiteren Anstieg des Stromflusses und dadurch zu einer Temperaturerhöhung und lokalem ,annealing“" [34]. Durch Elektromigration kommt es in überlasteten MTJs zu einer vollständigen Zerstörung. Laut Schäfers et al. werden Cobalt und Eisen durch das elektrische Feld aus ihrer ursprünglichen Position herausgetrieben, während Kupfer in Richtung des Elektronenstroms mitgerissen wird; dies führt zu einer vollständigen Zerstörung des MTJ [122].

Ob die „pinhole“-Bildung vornehmlich an Bor-kontaminierten Korngrenzen der MgO-Tunnelbarriere stattfindet, konnte im Rahmen dieser Arbeit nicht untersucht werden und wäre Gegenstand zukünftiger Untersuchungen.

Sollte diese ,pinhole“-Bildung mit Bor-kontaminierten Korngrenzen zusammenhängen, müsste Bor in der $\mathrm{MgO}$-Tunnelbarriere $\mathrm{zu}$ finden sein. In den untersuchten Modellsystemproben war dies nicht der Fall. In diesen Proben wurde eine $6 \mathrm{~nm}$ dünne $\mathrm{MgO}$-Schicht mittels Elektronenstrahlverdampfen auf einem MgO-Substrat aufgebracht. Rf-gesputterte MgO-Tunnelbarrieren zeigten in der Literatur bereits signifikante $\mathrm{B}$ - oder $\mathrm{BO}_{\mathrm{x}}$-Konzentrationen an den Grenzflächen zu den CoFeB-Elektroden [30], die bei elektronenstrahlverdampften $\mathrm{MgO}$-Barrieren nicht auftrat [50, 31]. 
Der Einfluss dieser beiden unterschiedlichen MgO-Depositionsmethoden auf die Bor-Verteilung nach dem Auslagern wurde mittels HRTEM und EELS an funktionsfähigen MTJs untersucht, um den Einfluss von Korngrenzen in texturierten MgO-Tunnelbarrieren zu berücksichtigen. Beide MTJs weisen homogene, kristalline MgO-Tunnelbarrieren mit scharfen Grenzflächen zu den kristallisierten CoFeB-Elektroden auf. EELS-Messungen an der Probe mit der elektronenstrahlverdampften MgO-Schicht bestätigen die Ergebnisse aus dem Modellsystem: Das Bor ist, in metallischer Form, hauptsächlich im Bereich über der CoFe-Schicht, in der oberen Ta-Deckschicht und nicht im MgO oder an dessen Grenzflächen lokalisiert. Dagegen ist in der Probe mit der rf-gesputterten MgO-Schicht in beiden Elektroden noch ein Borsignal messbar. Darüber hinaus konnte auch in der MgO-Schicht selbst Bor nachgewiesen werden. Der leicht andere Schichtaufbau der Proben kann die Borkonzentration im $\mathrm{MgO}$ allein nicht erklären, da diese Konzentration sonst schon in der Modellsystemmessung aufgefallen wäre.

Im Gegensatz zum Bor im CoFeB oder im Ta, in denen das Bor in metallischer Form vorliegt, ist das Bor im $\mathrm{MgO}$ als $\left[\mathrm{BO}_{3}\right]^{3-}$, also in einer trigonalen Anordnung mit Sauerstoff, zu finden [31, 85].

Ursächlich für die Anreicherung von Bor in der [rf]-MgO-Schicht könnte die defektreichere MgO-Schicht sein: die auf lokale Veränderungen sehr empfindlich reagierende ELNES-Messung [119, 31] zeigte weniger ausgeprägtere Maxima und Minima in dieser Probe. Einen Hinweis auf die Art der Defekte liefert der beobachtete „pre-peak“ in der ELNES der O-K-Kante zwischen 530 und 535 eV der rf-gesputterten MgO-Barriere, die in dieser Form auch schon bei Cha et al. [31] gefunden wurde. Laut Berechnungen von Velev et al. [120] deutet dieser Peak auf das Vorhandensein von ,gap states“ in der Bandstruktur des MgO hin, welche auf Sauerstoffleerstellen zurückzuführen sind. Eine EELS-Messung der stöchiometrischen Zusammensetzung der MgO-Tunnelbarriere sowohl in der [rf]- als auch in der [eb]-Probe führten aufgrund der großen Fehler dieser Methode von 10-20 \% [54] zu keinem Ergebnis und sollte in Zukunft systematisch untersucht werden.

Die Berechnungen von Velev et al. zeigten darüber hinaus, dass Sauerstoffleerstellen in der Barriere zu einer drastischen Verringerung des TMR führen können [120]. Dort zeigte sich, dass der TMR durch Sauerstoffleerstellen von $1800 \%$ auf $800 \%$ reduziert wird. Trotzdem zeigten beide hier untersuchten MTJs in vorherigen Messungen einen TMR von $200 \%$. D.h., dass weder die erhöhte Borkonzentration im $\mathrm{MgO}$ noch die beobachteten ,gap states“ den TMR in diesen Proben limitieren. Die gefundene Boranreicherung in der [rf]MTJ ist in der Barriere senkrecht zu dieser homogen verteilt, wie es auch bei Harnchana et al. [121] der Fall war. Die reine Anlagerung des Bor an den Grenz- 
flächen der Barriere, die in der Literatur öfters gefunden wurde [32, 25, 30], konnte in den hier durchgeführten Untersuchungen nicht bestätigt werden.

Sowohl die chemischen Analysen als auch die Ergebnisse der strukturellen Untersuchungen der amorph/kristallinen-Grenzfläche sprechen für eine Borfreie Grenzfläche bei eb-deponierten MgO-Schichten, da Bor-kontaminierte Grenzflächen einen deutlich größeren Fe-O-Abstand aufweisen [24]. Ob das Bor in den rf-gesputterten MgO-Schichten nur an Korngrenzen lokalisiert ist oder homogen verteilt ist, konnte in dieser Arbeit nicht aufgelöst werden und wäre Ansatzpunkt weiterer Untersuchungen. Da die eb-deponierten MgO-Tunnelelemente kein Bor in der Barriere aufwiesen, stellt sich die Frage, ob diese bei einer elektrischen Belastung stabiler sind als rf-gesputterte MTJs. 
5. Zusammenfassende Diskussion und Ausblick 


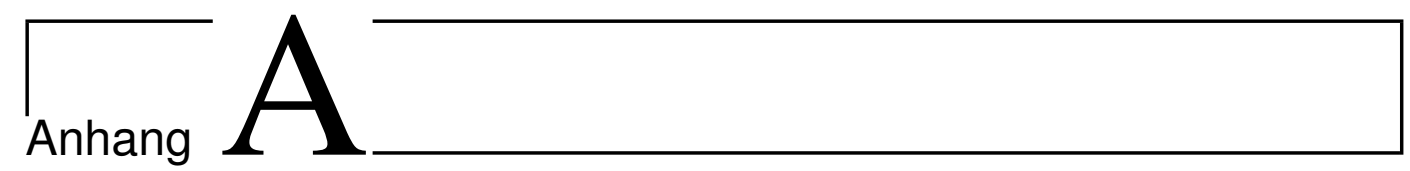

\section{Atomares Streupotential}

Das atomare Streupotential wurde nach Weickenmeier und Kohl [124] auf Basis der Gleichung

$$
f(s)=s^{-2} \sum_{i=1}^{6} A_{i}\left(1-\exp \left(-B_{i} s^{2}\right)\right)
$$

mit

$$
A_{1}=0,02395 \cdot Z /(3(1+V)) \text { und } A_{4}=V \cdot A_{1}
$$

berechnet. Dabei gilt:

$$
A_{1}=A_{2}=A_{3} \text { und } A_{4}=A_{5}=A_{6}
$$

Als Parameter dienten die bei Weickenmeier und Kohl [124] angegebenen Parameter:

\begin{tabular}{ccccccccc}
\hline & $\mathrm{Z}$ & $\mathrm{V}$ & $B_{1}$ & $B_{2}$ & $B_{3}$ & $B_{4}$ & $B_{5}$ & $B_{6}$ \\
\hline \hline $\mathrm{B}$ & 5 & 0,5 & 0,3314 & 2,975 & 34,01 & 35,98 & 36,68 & 60,81 \\
$\mathrm{Fe}$ & 26 & 0,5 & 0,4470 & 6,894 & 6,903 & 0,05691 & 3,026 & 70,87 \\
$\mathrm{Co}$ & 27 & 0,5 & 0,1071 & 3,636 & 7,558 & 1,280 & 5,140 & 67,16 \\
\hline
\end{tabular}

Tabelle A.1.: Acht Parameter für die Anpassung an das elastische atomare Streupotential aus [124] für die in dieser Arbeit untersuchten Materialien: B, Fe, Co.

Das hier angegebene Streupotential wird auch bei EMS für die Simulation der HRTEM-Abbildungen genutzt. Aus der Auftragung der Formel A.1 ist sichtbar (s. Abb. A.1), dass sowohl Eisen (schwarz) als auch Cobalt (rot) ein 
größeres Streupotential aufweisen als das leichtere Bor (blau). Das Streupotential von Fe und Co unterscheiden sich nur geringfügig, weshalb das mittlere projizierte Potential im $\mathrm{CoFeB}$ mit dem Streupotential von Fe gebildet wird.

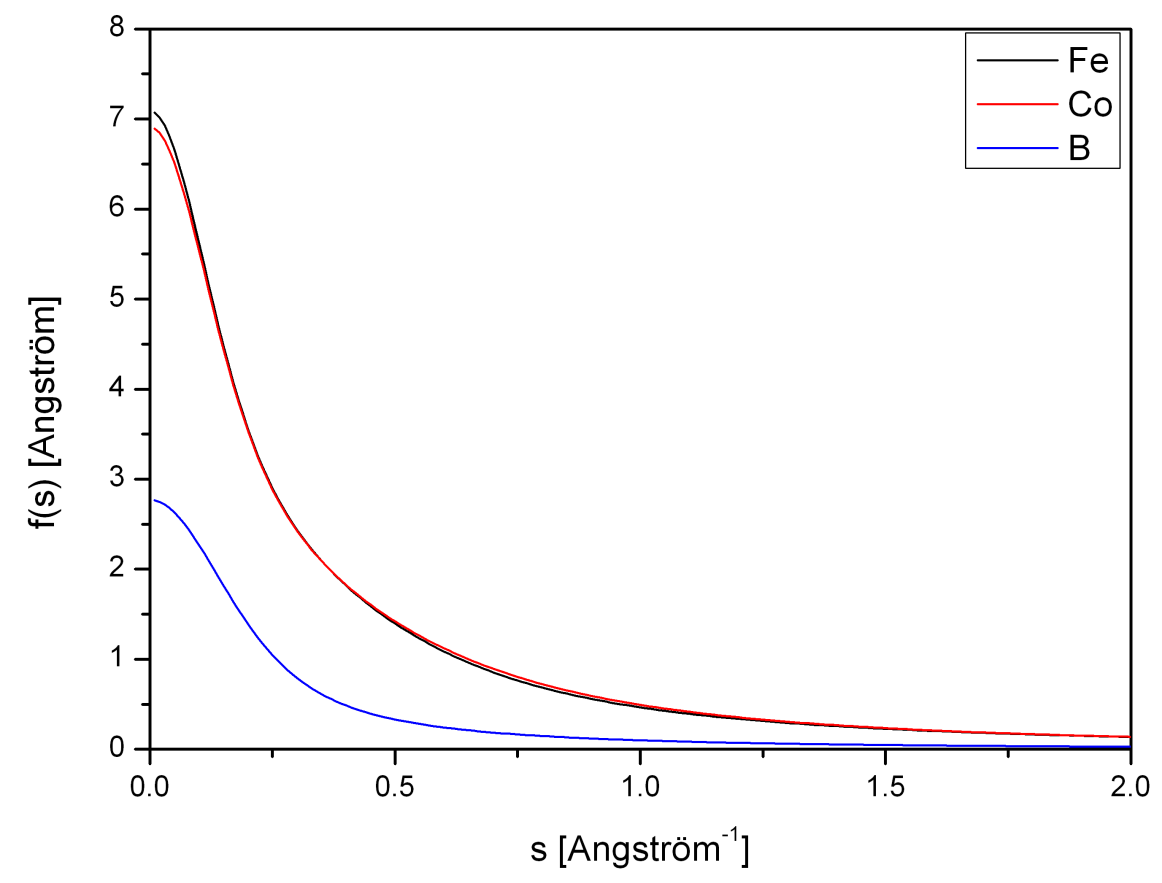

Abbildung A.1.: Das atomare Streupotential von Eisen (schwarz), Cobalt (rot), Bor (blau), berechnet nach Gleichung A.1, zeigt ein nahezu identisches Streupotential für Cobalt und Eisen sowie ein geringeres Streupotential für Bor. Nach 124. 


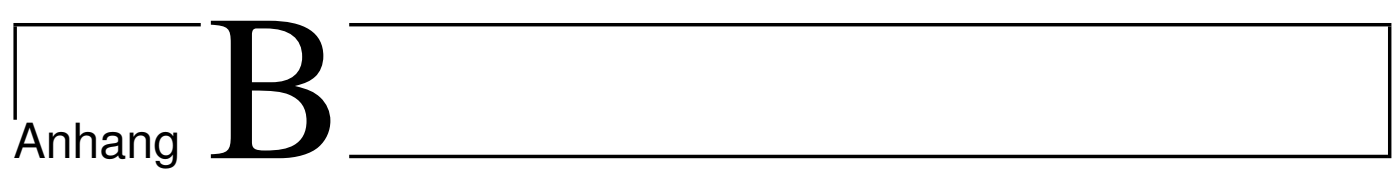

\section{Probenpräparation}

Im Folgenden sollen die Proben vorgestellt werden, die bei den einzelnen Untersuchungen verwendet wurden. Grundsätzlich kamen dabei zwei verschiedene Depositionsmethoden zur Anwendung. Das Elektronenstrahlverdampfen und die Sputterdeposition.

- Bei der Sputterdeposition wird das aufzubringende Material durch Beschuss mit hochenergetischen Ionen aus einem Target herausgelöst und in die Gasphase gebracht. Die hochenergetischen Ionen werden mit einem Niederdruckplasma aus Edelgas, zumeist Argon, erzeugt und mit einem elektrischen Feld in Richtung des Targets beschleunigt. Dabei liegt am Target eine negative Bias-Spannung an; das Substrat bildet die positive Elektrode. Die hochenergetischen Ionen geben ihre Energie in Stoßkaskaden an das Target ab und lösen dadurch Atome heraus, die sich wieder auf dem Substrat ablagern. Dieses DC-Sputtern genannte Verfahren findet häufig bei elektrisch leitfähigen Target-Materialien Anwendung; elektrisch isolierende Materien würden sich im elektrischen Feld aufladen und somit die Ionen abbremsen. Für elektrisch isolierende Materialien wird deshalb das Hochfrequenz-Sputtern (kurz: rf-sputtern) angewendet, bei dem eine hochfrequente Wechselspannung angelegt wird. Eine in der Mikroelektronik häufig verwendete Methode zur Aufbringung von Metallschichten ist das Magnetronsputtern. Bei diesem Verfahren wird über dem Target-Material ein zusätzliches Magnetfeld erzeugt, das die Elektronen des Plasmas auf eine Spiralbahn ablenkt, die Ionen jedoch nur geringfügig beeinflusst. Dort, wo die Magentfeldlinien parallel zur Targetoberläche verlaufen, ist die Elektronendichte am größten und bewirkt eine erhöhte Ionisation. Unter diesen Bereichen ist der Sputterabtrag dann deutlich erhöhter. 
Allen Methoden ist gemein, dass die Target-Atome auf dem Weg zum Substrat einen Teil ihrer kinetischen Energie durch Stöße an das Sputtergas abgeben. Dadurch kann bei Legierungen die stöchiometrische $\mathrm{Zu}$ sammensetzung auf dem Substrat eine andere sein als ursprünglich im Target vorhanden.

- Beim Elektronenstrahlverdampfen erhitzt ein Elektronenstrahl das zu verdampfende Material unter Ultrahochvakuum in einem Tiegel. Da die Rückseite des Tiegels gekühlt wird, sorgt der entstehende Temperaturgradient dafür, dass nur der Bereich, in dem der Elektronenstrahl auftrifft, eine Temperatur erreicht, die das Material verdampfen lässt. Da hier lokal eine hohe Energiedichte erzeugt werden kann, eignet sich dieses Verfahren um alle bekannten Feststoffe zu verdampfen. Da die einzelnen Bestandteile von Legierungen in der Regel unterschiedliche Dampfdrücke aufweisen, würde beim direkten Verdampfen dieser Materialien auf dem Substrat eine andere Zusammensetzung vorliegen. Deshalb wird in einem solchen Fall ein Ko-Verdampfen der einzelnen Bestandteile aus unterschiedlichen Tiegeln vorgenommen.

\section{B.1. Herstellung der $\mathrm{c}-\mathrm{MgO} / \mathrm{c}-\mathrm{CoFe}-$ Probe}

Die Probe für die Untersuchung der kristallin/kristallinen Grenzfläche (siehe Abschnitt 4.1.1) zwischen $\mathrm{MgO}$ und $\mathrm{CoFe}$ wurde auf einem einkristallinen MgO-Substrat hergestellt, auf dem mittels Elektronenstrahlverdampfens eine $6 \mathrm{~nm}$ dicke MgO-Schicht aufgebracht wurde, damit eine glatte Oberfläche entsteht. Die Substrattemperatur betrug dabei $350{ }^{\circ} \mathrm{C}$ bei einem Sauerstoffpartialdruck in der Kammer von $1,7 \cdot 10^{-8}$ mbar. Über dieser Schicht wurde bei Raumtemperatur eine $100 \mathrm{~nm}$ dicke Schicht $\mathrm{Co}_{20} \mathrm{Fe}_{60} \mathrm{~B}_{20}$ gesputtert. Um Oxidationen der Probe zu vermeiden wurde anschließend noch eine $10 \mathrm{~nm}$ Tantalschicht und eine $3 \mathrm{~nm}$ dicke Schicht Ruthenium aufgebracht. Im Anschluss wurde die Probe für 60 Minuten bei $450{ }^{\circ} \mathrm{C}$ ausgelagert, damit die CoFeBSchicht kristallisiert.

\section{B.2. Herstellung der $\mathrm{c}-\mathrm{MgO} / \mathrm{a}-\mathrm{CoFeB}-$ Probe}

Die Probe für die Untersuchung der kristallin/amorphen Grenzfläche (siehe Abschnitt 4.1.2 zwischen $\mathrm{MgO}$ und $\mathrm{CoFeB}$ wurde im Wesentlichen genauso hergestellt wie die Probe für die Untersuchung der kristallin/kristallinen 
Grenzfläche in Abschnitt B.1. Hier wurde ebenfalls auf ein einkristallines MgOSubstrat mittels Elektronenstrahlverdampfens eine $6 \mathrm{~nm}$ dicke MgO-Schicht aufgebracht. Die Substrattemperatur lag bei $350{ }^{\circ} \mathrm{C}$ bei einem Sauerstoffpartialdruck in der Kammer von $1,7 \cdot 10^{-8}$ mbar. Nachdem diese Schicht auf Raumtemperatur abgekühlt ist, wurde hierauf eine $100 \mathrm{~nm}$ dicke Schicht $\mathrm{Co}_{20} \mathrm{Fe}_{60} \mathrm{~B}_{20}$ gesputtert und diese anschließend mit einer $3 \mathrm{~nm}$ dünnen Schicht Ruthenium vor Oxidation geschützt. Eine Auslagerung fand bei dieser Probe nicht statt.

\section{B.3. Probeherstellung für die Untersuchung der Deckschichtabhängigkeit}

Für die Untersuchung der Bordiffusion und Kristallisation des CoFeB in Abhängigkeit vom Deckschichtmaterial (siehe Abschnitt 4.2.1) wurden mehrere Modellsystemproben hergestellt. Die Grundlage dieser Proben bestand wie in den vorangegangenen Untersuchungen aus einer per Elektronenstrahlverdampfen aufgebrachten $6 \mathrm{~nm}$ dicken $\mathrm{MgO}-\mathrm{Schicht}$ auf einem einkristallinen MgOSubstrat. Darüber wurden entweder eine $5 \mathrm{~nm}$ oder $100 \mathrm{~nm}$ dicke $\mathrm{Co}_{20} \mathrm{Fe}_{60} \mathrm{~B}_{20}$ gesputtert. Über diese Schicht wurde dann die Deckschicht aufgetragen, die entweder aus einer $10 \mathrm{~nm} \mathrm{Ru-Schicht,} \mathrm{oder} \mathrm{aber} \mathrm{einer} 10 \mathrm{~nm}$ Ta-Schicht und eine $3 \mathrm{~nm} \mathrm{Ru-Schicht} \mathrm{bestand.} \mathrm{Ru} \mathrm{wurde} \mathrm{dabei} \mathrm{mittels} \mathrm{Elektronenstrahlver-}$ dampfens und Ta mittels [rf]-Sputtern aufgetragen. Die Ru-Schicht sollte das Ta dabei vor dem oxidieren schützen. Diese Probe wurde als Ta-Deckschichtprobe bezeichnet, da die Ru-Schicht hier keinen Einfluss auf das CoFeB ausübt. Daraus ergeben sich insgesamt vier Proben (s. Abb. B.1), die alle für $60 \mathrm{Mi}-$ nuten bei $450{ }^{\circ} \mathrm{C}$ ausgelagert wurden, um eine Kristallisation des CoFeB zu ermöglichen.

\section{B.4. Herstellung der funktionalen MTJs mit verschiedenen $\mathrm{MgO}$-Depositionsmethoden}

Die Schichtsysteme für die Untersuchung des Einflusses der MgO-Depositionsmethode (siehe Abschnitt 4.2.2) sind, verglichen mit den vorangegangenen Modellsystemproben, wesentlich komplexer. Die [rf]-Probe wurde an der Universität Bielefeld von der Arbeitsgruppe Thin Films and Physics of Nanostructures um Andy Thomas hergestellt und weist ausgehend vom $\mathrm{SiO}_{2}$-Substrat folgende Schichtreihenfolge auf: $\mathrm{Ta}(5) / \mathrm{Ru}(30) / \mathrm{Ta}(30) / \mathrm{Ru}(10) / \mathrm{CoFeB}(4) / \mathrm{MgO}(2,1) /$ $\mathrm{CoFeB}(1,5) / \mathrm{Ta}(5) / \mathrm{Ru}(20)$. Sie wurde für 60 Minuten bei $400{ }^{\circ} \mathrm{C}$ ausgelagert. Die Angabe in Klammern bezeichnet die Schichtdicken in nm. Der Druck in 


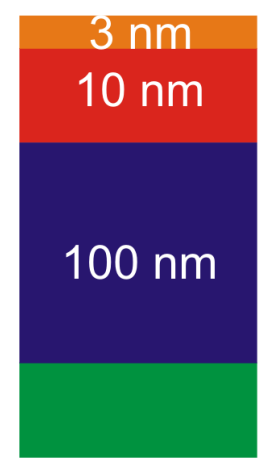

$\mathrm{MgO}$



- CoFeB

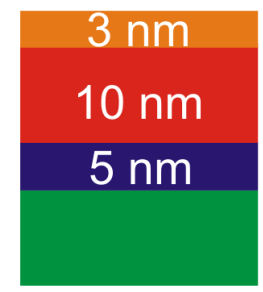

Ta

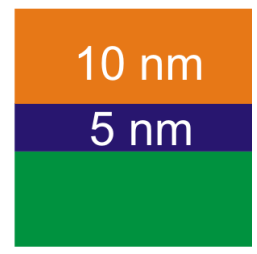

$\mathrm{Ru}$

Abbildung B.1.: Übersicht der auf $M g O$ Substrat hergestellten Proben mit 5 bzw 100 nm dicker CoFeB Schicht und darüberliegender Deckschicht aus Ta oder Ru.

der Kammer betrug $1 \cdot 10^{-9}$ mbar.

Die Schichtreihenfolge der an der Universität Göttingen hergestellten [eb]Probe ist etwas anders: $\mathrm{SiO}_{2}$ (Substrat)/ Ta(5)/CoFeB $(2,5) / \mathrm{MgO}(2,3) /$

$\mathrm{CoFeB}(5,4) / \mathrm{Ta}(5) / \mathrm{Ru}(3)$. Sie wurde für 60 Minuten bei $375{ }^{\circ} \mathrm{C}$ ausgelagert.

\section{B.5. Herstellung der Proben für die Untersuchung des Durchbruchverhaltens}

Die für die Untersuchung des Durchbruchverhaltens (siehe Abschnitt 4.3) hergestellten Proben wurden ebenfalls an der Universität Bielefeld hergestellt und strukturiert. Diese MTJs besitzen die Schichtfolge: Ta(5)/CuN(90)/Ta(5)/ $\operatorname{PtMn}(20) / \mathrm{CoFe}(2,2) / \mathrm{Ru}(0,8) / \mathrm{CoFeB}(2) / \mathrm{MgO}(1,1) / \mathrm{CoFeB}(1,5) / \mathrm{Ta}(10) /$ $\mathrm{CuN}(30) / \mathrm{Ru}(7)+(\mathrm{Ta}(5) / \mathrm{Au}(60))$. Die beiden letzten Elemente dienten nur der elektrischen Kontaktierung der Probe.

Dieser Schichtstapel wurde für 90 Minuten bei $360{ }^{\circ} \mathrm{C}$ in einem Magnetfeld von $1 \mathrm{~T}$ ausgelagert und mittels Elektronenstrahllithographie und Ionenätzen strukturiert. Die MTJ Elemente hatten eine elliptische Form der Größe 380 nm x $150 \mathrm{~nm}$ und wurden mittels FIB Zielpräparation gedünnt. 


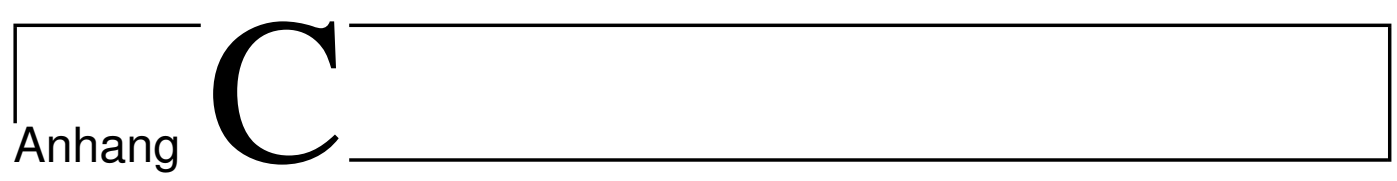

\section{Intensitätsprofile und \\ Grenzflächenabbildungen}

\section{C.1. Profile der Grenzflächenposition 2}

In den Abbildungen C.1 und C.2 sind die simulierten und experimentellen Intensitätsprofile dargestellt. Die simulierten Intensitätsprofile wurden unter der Annahme der $\mathrm{MgO} / \mathrm{CoFeB}-$ Grenzfläche an Position 2 iterativ bestimmt. 



Abbildung C.1.: Simulierte (rot) und experimentelle (schwarz) Profile für die Abbildungen 1-10 mit einem Defokusbereich zwischen $\Delta f=28 \mathrm{~nm}$ bis $\Delta f=1 \mathrm{~nm}$. 

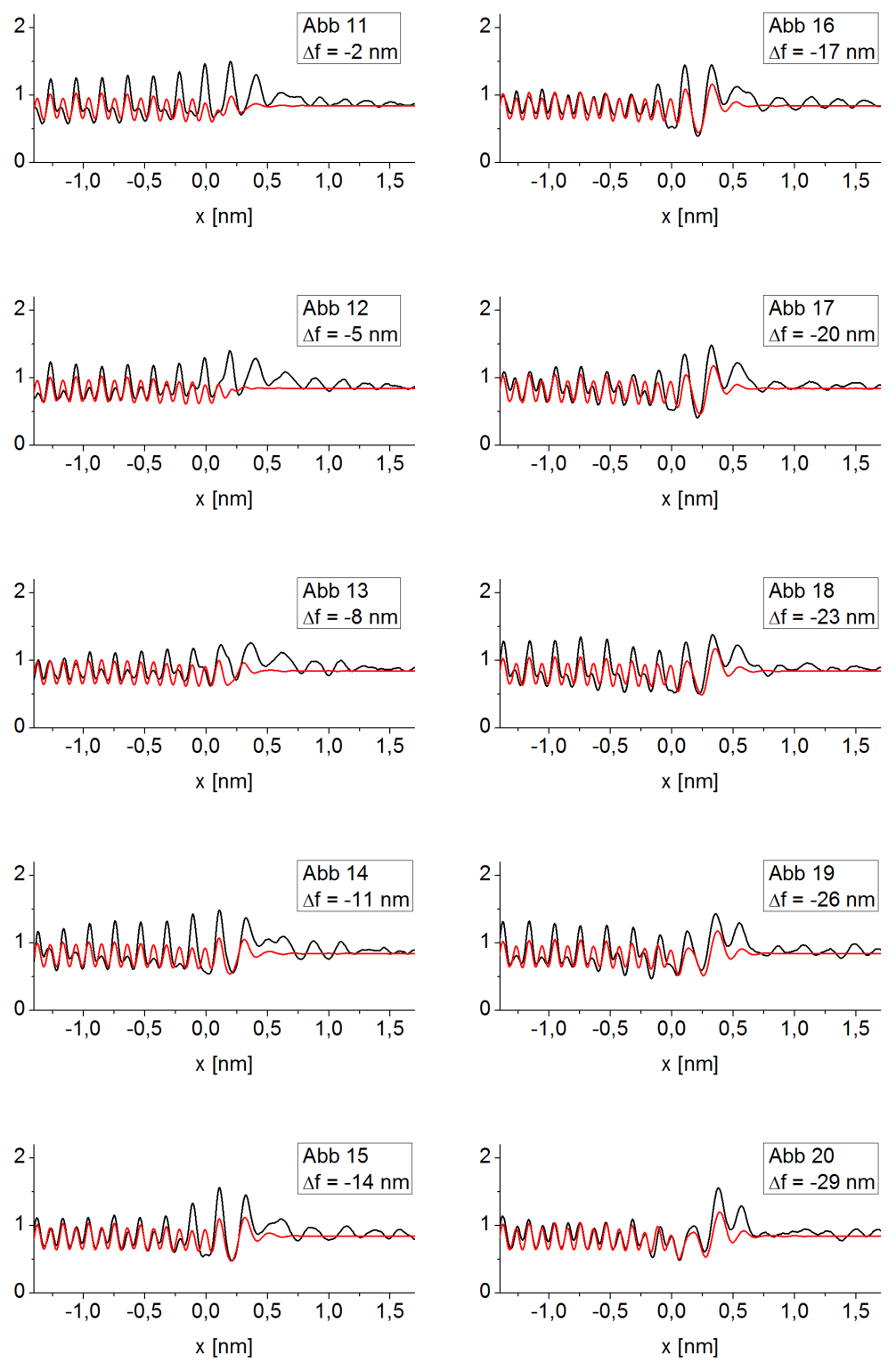

- Exp. $\longrightarrow$ Sim.

Abbildung C.2.: Simulierte (rot) und experimentelle (schwarz) Profile für die Abbildungen 11-20 mit einem Defokusbereich zwischen $\Delta f=-2 \mathrm{~nm}$ bis $\Delta f=-29 \mathrm{~nm}$. 


\section{C.2. Ergebnisse der iterativen Anpassung mit 10 Parametern}

Im Folgenden sind die Intensitätsprofile und Abbildungen für die Anpassung mit zehn Parametern unter Berücksichtigung einer Stufe bei $n_{P o s 2} / n_{P o s 3}=$ 13/13 dargestellt. Die Abbildungen C.4 und C.5zeigen den Vergleich der Anpassung mit 10 Parametern (rot) zu den experimentellen Intensitäten (schwarz) und den mit acht Parametern erstellten Daten (blau). Die blaue Linie in Abbildung C.3 kennzeichnet die Grenzflächenposition 2 welche die ersten 13 Atomlagen aufweisen. Die Grenzflächenposition 3 der folgenden 13 Atomlagen, befindet sich eine Atomlage von dieser Linie nach links versetzt.

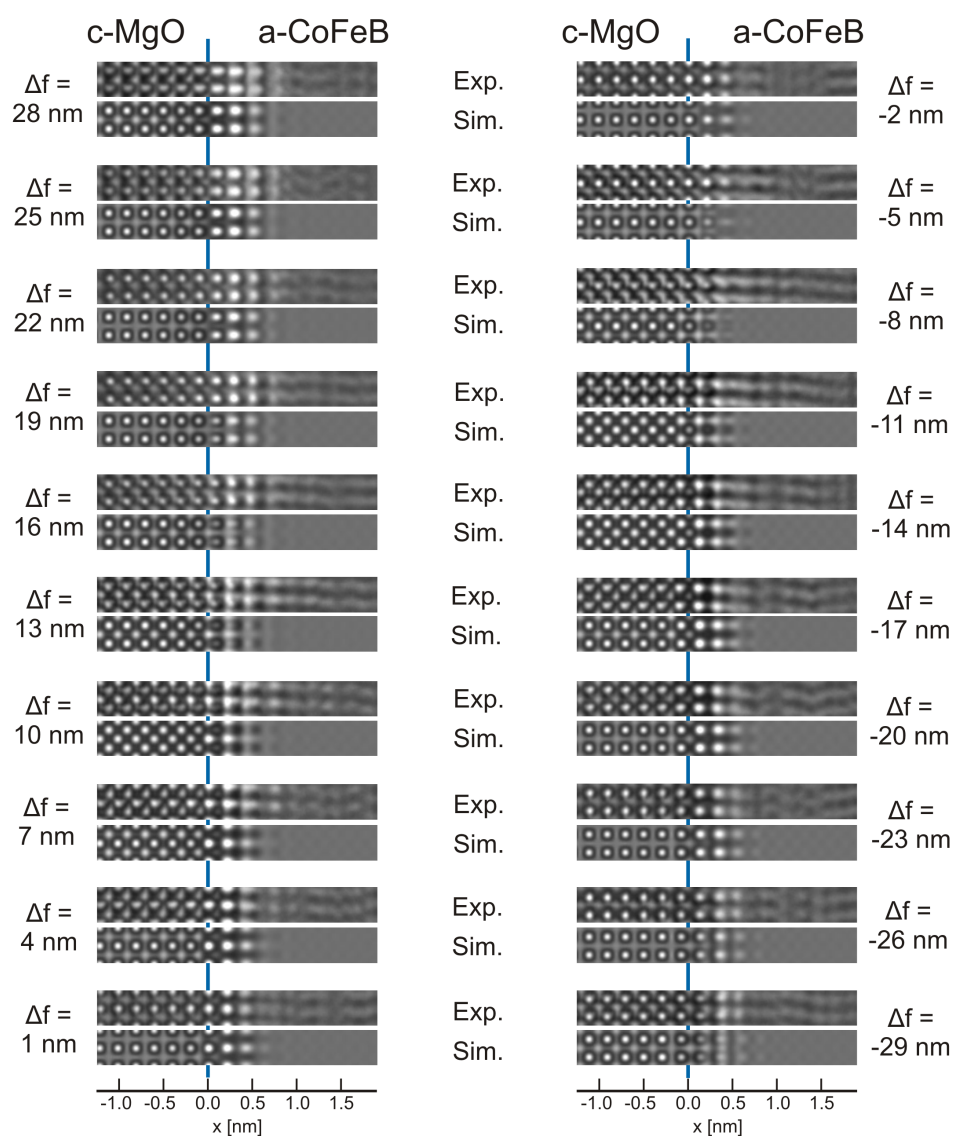

Abbildung C.3.: Darstellung der experimentellen Grenzflächenabbildungen im Vergleich zu den simulierten Abbildungen unter Berücksichtigung einer Grenzflächenstufe und mit 10 Parametern. Die blaue Linie kennzeichnet die zweite Grenzflächenposition, die dritte Grenzflächenposition liegt eine Atomlage weiter links. 



Abbildung C.4.: Intensitätsprofile der Abbildungen 1-10 mit einem Defokusbereich zwischen $\Delta f=28 \mathrm{~nm}$ bis $\Delta f=1 \mathrm{~nm}$. Die experimentellen Daten werden durch eine schwarze Linie repräsentiert. In rot sind die Intensitätsprofile der mit 10 Parametern und in blau die mit acht Parametern konstruierte simulierten Profile dargestellt. 

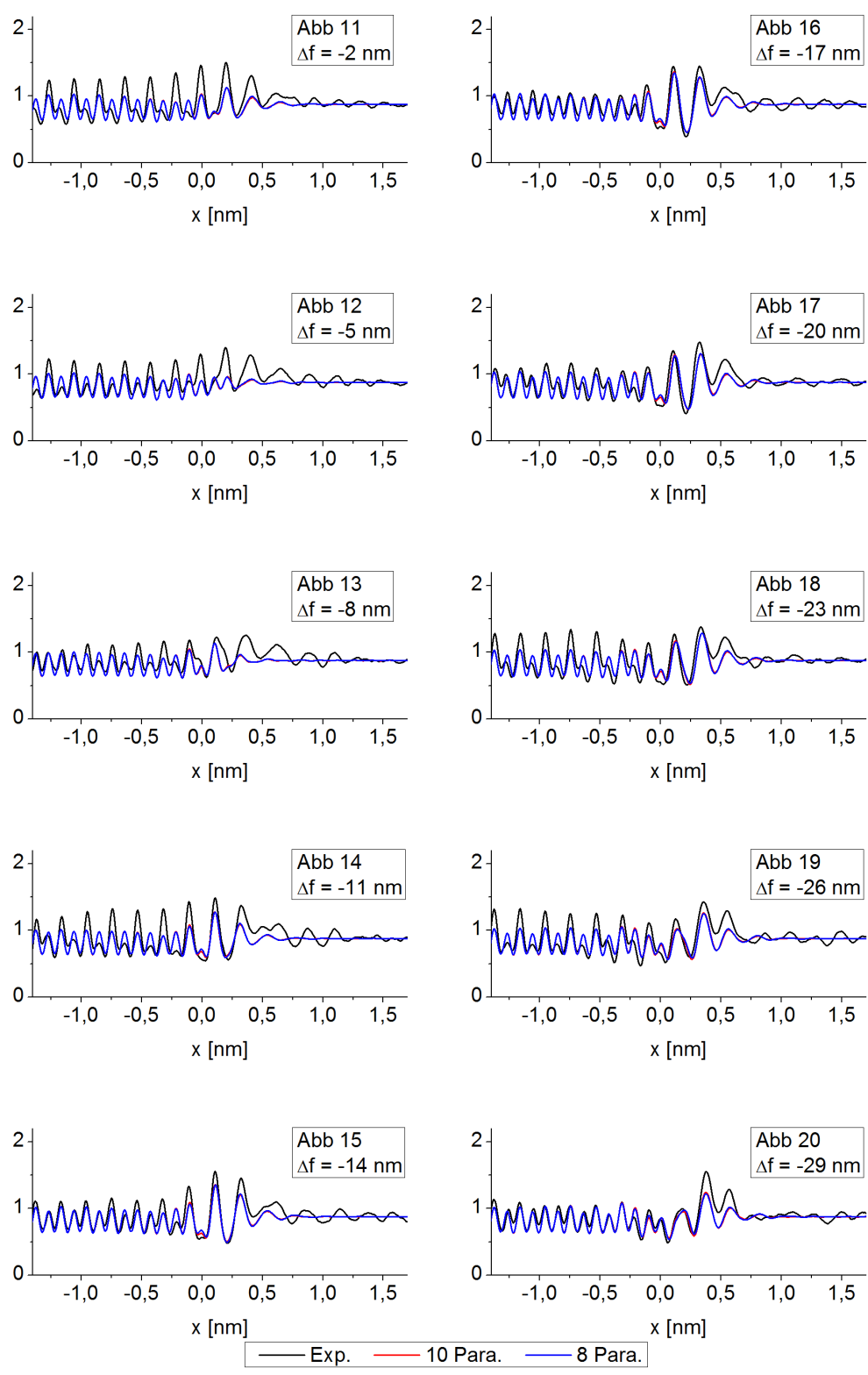

\begin{abstract}
Abbildung C.5.: Intensitätsprofile der Abbildungen 11-20 mit einem Defokusbereich zwischen $\Delta f=-2 \mathrm{~nm}$ bis $\Delta f=-29 \mathrm{~nm}$. Die experimentellen Daten werden durch eine schwarze Linie repräsentiert. In rot sind die Intensitätsprofile der mit 10 Parametern und in blau die mit acht Parametern konstruierte simulierten Profile dargestellt.
\end{abstract}



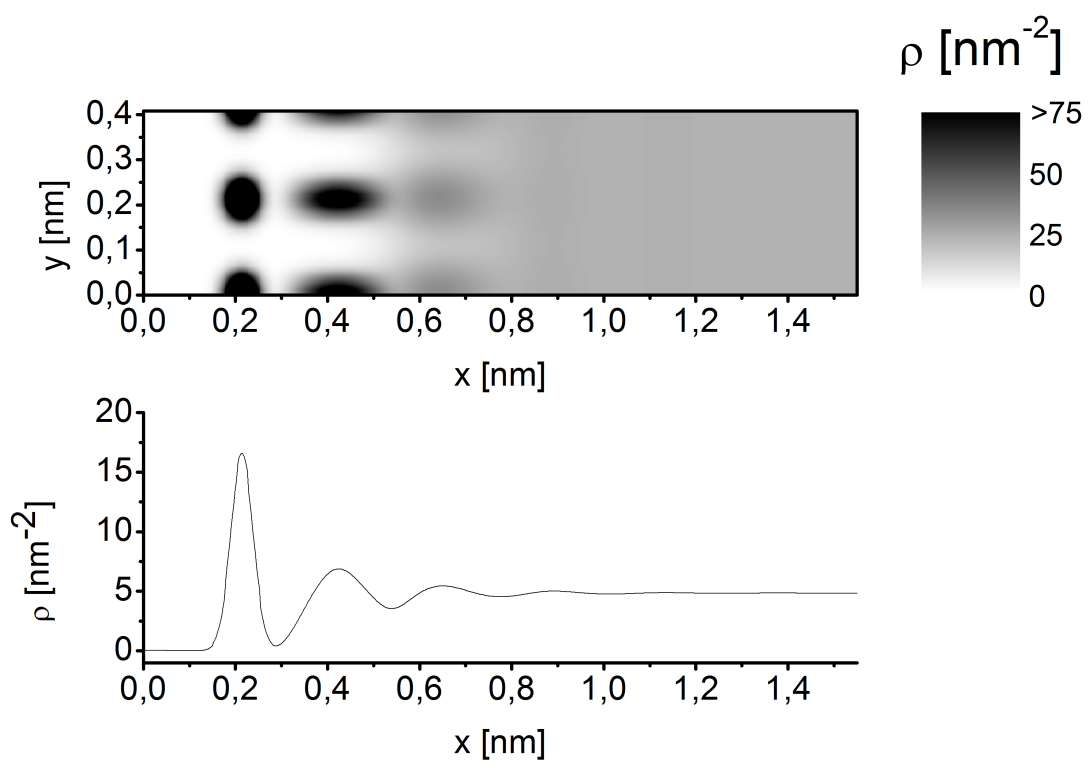

(a)



(b)

Abbildung C.6.: a) Oben: Darstellung der zweidimensionalen Verteilungsfunktion für die Berechnung der simulierten Abbildungen mit zehn Parametern. Unten: Eindimensionales Profil der atomaren Dichte $\rho$. b) Bestimmung der Ausdehnung des beeinflussten Übergangsbereiches zwischen kristallinem $\mathrm{MgO}$ und amorphem CoFeB bei einer iterativer Anpassung mit zehn Parametern. 
C. Intensitätsprofile und Grenzflächenabbildungen 


\section{Literaturverzeichnis}

[1] M. Julliere, Tunneling between Ferromagnetic Films, Physics Letters 54A, No. 3, 225 (1975).

[2] S. Yuasa und D. D. Djayaprawira, Giant tunnel magnetoresistance in magnetic tunnel junctions with a crystalline $M g O(001)$ barrier, Journal of Physics D: Applied Physics 40, R337 (2007).

[3] M. Hosomi, H. Yamagishi, T. Yamamoto, K. Bessho, Y. Higo, K. Yamane, H. Yamada, M. Shoji, H. Hachino, C. Fukumoto, H. Nagao und H. Kano, A novel nonvolatile memory with spin torque transfer magnetization switching: spin-ram, in Electron Devices Meeting, 2005. IEDM Technical Digest. IEEE International, Seiten 459-462, 2005.

[4] S. Ikeda, H. Hayakawa, Y. Ashizawa, Y. M. Lee, K. Miura, H. Hasegawa, M. Tsunoda, F. Matsukara und H. Ohno, Tunnel magnetoresistance of $604 \%$ at $300 \mathrm{~K}$ by suppression of Ta diffusion in $\mathrm{CoFeB} / \mathrm{MgO} / \mathrm{CoFeB}$ pseudo-spin-valves annealed at high temperature, Appl. Phys. Lett. 93 (2008).

[5] T. Miyazaki und N. Tezuka, Giant magnetic tunneling effect in $\mathrm{Fe} / \mathrm{Al}_{2} \mathrm{O}_{3} / \mathrm{Fe}$ junction, Journal of Magnetism and Magnetic Materials 139 (1995).

[6] J. S. Moodera, L. R. Kinder, T. M. Wong und R. Meservey, Large Magnetoresistance at Room Temperature in Ferromagnetic Thin Film Tunnel Junctions, Phys. Rev. Let. 74, No. 16, 3273 (1995).

[7] W. H. Butler, X. G. Zhang, T. C. Schulthess und J. M. MacLaren, Spindependent tunneling conductance of $\mathrm{Fe} / \mathrm{MgO} / \mathrm{Fe}$ sandwiches, Physical Review B 63, 054416 (2001). 
[8] J. Mathon und A. Umerski, Theory of tunneling magnetoresistance of an epitaxial $\mathrm{Fe} / \mathrm{MgO} / \mathrm{Fe}(001)$ junction, Physical Review B 63, 220403 (2001).

[9] M. Bowen, V. Cros, F. Petroff, A. Fert, C. Martínez Boubeta, J. L. Costa-Krämer, J. V. Anguita, A. Cebollada, F. Briones, J. M. de Teresa, L. Morellón, M. R. Ibarra, F. Güell, F. Peiró und A. Cornet, Large magnetoresistance in $\mathrm{Fe} / \mathrm{MgO} / \mathrm{FeCo}(001)$ epitaxial tunnel junctions on GaAs(001), Applied Physics Letters 79, 1655 (2001).

[10] D. Djayaprawira, K. Tsunekawa, M. Nagai, H. Maehara, S. Yamagata, N. Watanabe, S. Yuasa, Y. Suzuki und K. Ando, 230 \% roomtemperature magnetoresistance in $\mathrm{CoFeB} / \mathrm{MgO} / \mathrm{CoFeB}$ magnetic tunnel junctions, Appl. Phys. Lett. 86 (2005).

[11] C. Heiliger, M. Gradhand, P. Zahn und I. Mertig, Tunneling Magnetoresistance on the Subnanometer Scale, Physical Review Letters 99, 066804 (2007).

[12] N. I. Borgardt, B. Plikat, W. Schröter und M. Seibt, Regularities of averaged HREM-images of crystalline-amorphous interfaces, Bulletin of the Russian Academy of Sciences 61, 1544 (1997).

[13] N. I. Borgardt, B. Plikat, M. Seibt und W. Schröter, Analysis of high resolution transmission electron microscope images of crystallineamorphous interfaces, Ultramicroscopy 90, 241 (2002).

[14] N. I. Borgardt, B. Plikat, M. Seibt und W. Schröter, The Effect of the Translational Symmetry of Crysalline Silicon on the Structure of Amorphous Germanium in the Interfacial Region, Surface thin films 49, 225 (2004).

[15] N. I. Borgardt, B. Plikat, W. Schröter, M. Seibt und T. Wagner, Atomic structure of the interface between silicon (111) and amorphous germanium, Phys. Rev. B 70, 195307 (2004).

[16] J. M. Cowley und A. F. Moodie, The scattering of electrons by atoms and crystals. I. A new theoretical approach, Acta Crystallographica 10, 609 (1957).

[17] D. F. Lynch und M. A. O'Keefe, n-Beam lattice images. II. Methods of calculation, Acta Crystallographica Section A 28, 536 (1972). 
[18] P. Goodman und A. F. Moodie, Numerical evaluations of $N$-beam wave functions in electron scattering by the multi-slice method, Acta Crystallographica Section A 30, 280 (1974).

[19] K. Ishizuka und N. Uyeda, A new theoretical and practical approach to the multislice method, Acta Crystallographica Section A 33, 740 (1977).

[20] D. van Dyck, Image calculations in high-resolution electron microscopy: problems, progress, and prospects, Advances in electronics and electron physikcs 65, 295 (1985).

[21] K. Thiel, Strukturelle Untersuchung der amorph/krisallinen Grenzfläche mittels quantitativer hochauflösender Transmissionselektronenmikroskopie an den Systemen a-Si/c-Si und a-Ge/c-Si, Dissertation, GeorgAugust-Universität Göttingen, 2006.

[22] B. Plikat, Hochauflösende Transmissionselektronenmikroskopie an den kristallin-amorphen Grenzfläche c-Si / a-Ge und c-Si / a-SiO ${ }_{2}$, Dissertation, Universität Göttingen, 1998.

[23] S. Yuasa, Y. Suzuki, T. Katayama und K. Ando, Characterization of growth and crystallization processes in $\mathrm{CoFeB} / \mathrm{MgO} / \mathrm{CoFeB}$ magnetic tunnel junction structure by reflective high-energy electron diffraction, Appl. Phys. Let. 87, 242503 (2005).

[24] J. D. Burton, S. S. Jaswal, E. Y. Tsymbal, O. N. Mryasov und O. G. Heinonen, Atomic and electronic structure of the CoFeB/MgO interface from first principles, Applied Physics Letters 89, 142507 (2006).

[25] S. V. Karthik, Y. K. Takahashi, T. Ohkubo, K. Hono, S. Ikeda und H. Ohno, Transmission electron microscopy investigation of CoFeB/MgO/CoFeB pseudospin valves annealed at different temperatures, Journal of Applied Physics 106, 023920 (2009).

[26] T. Miyajima, T. Ibusuki, S. Umehara, M. Sato, S. Eguchi, M. Tsukada und Y. Kataoka, Transmission electron microscopy study on the crystallization and boron distribution of $\mathrm{CoFeB} / \mathrm{MgO} / \mathrm{CoFeB}$ magnetic tunnel junctions with various capping layers, 94, 122501 (2009).

[27] Y. Lu, B. Lepine, G. Jezequel, S. Ababou, M. Alnot, J. Lambert, A. Renard, M. Mullet, C. Deranlot, H. Jaffres, F. Petroff und J.-M. George, Depth analysis of boron diffusion in $\mathrm{MgO} / \mathrm{CoFeB}$ bilayer by $\mathrm{x}^{-}$ ray photoelectron spectroscopy, Journal of Applied Physics 108, 043703 (2010). 
[28] S. V. Karthik, Y. K. Takahashi, T. Ohkubo, K. Hono, H. D. Gan, S. Ikeda und H. Ohno, Transmission electron microscopy study on the effect of various capping layers on $\mathrm{CoFeB} / \mathrm{MgO} / \mathrm{CoFeB}$ pseudo spin valves annealed at different temperatures, Journal of Applied Physics 111, 083922 (2012).

[29] K. Tsunekawa, M. Nagai, H. Maehara, S. Yamagata, D. Djayaprawira, N. Watanabe, S. Yuasa, Y. Suzuki und K. Ando, CoFeB $/ \mathrm{MgO} / \mathrm{CoFeB}$ magnetic tunnel junctions with high TMR ratio and low junction resistance, in Digests of the IEEE Int. Magnetics Conf, IEEE, 2005.

[30] D. K. Schreiber, Y. suk Choi, Y. Liu, A. N. Chiaramonti, D. N. Seidman und A. K. Petford-Long, Effects of elemental distributions on the behavior of MgO-based magnetic tunnel junctions, Journal of Applied Physics 109, 103909 (2011).

[31] J. J. Cha, J. C. Read, R. A. Buhrman und D. A. Muller, Spatially resolved electron energy-loss spectroscopy of electron-beam grown and sputtered $\mathrm{CoFeB} / \mathrm{MgO} / \mathrm{CoFeB}$ magnetic tunnel junctions, Applied Physics Letters 91, 062516 (2007).

[32] J. J. Cha, J. C. Read, W. F. Egelhoff, P. Y. Huang, H. W. Tseng, Y. Li, R. A. Buhrman und D. A. Muller, Atomic-scale spectroscopic imaging of $\mathrm{CoFeB} / \mathrm{Mg}-\mathrm{B}-\mathrm{O} / \mathrm{CoFeB}$ magnetic tunnel junctions, Applied Physics Letters 95, 032506 (2009).

[33] K. Komagaki, M. Hattori, K. Noma, H. Kanai, K. Kobayashi, Y. Uehara, M. Tsunoda und M. Takahashi, Influence of Diffused Boron Into $\mathrm{MgO}$ Barrier on Pinhole Creation in $\mathrm{CoFeB} / \mathrm{MgO} / \mathrm{CoFeB}$ Magnetic Tunnel Junctions, Magnetics, IEEE Transactions on 45, 3453 (2009).

[34] A. Thomas, V. Drewello, M. Schäfers, A. Weddemann, G. Reiss, G. Eilers, M. Münzenberg, K. Thiel und M. Seibt, Direct imaging of the structural change generated by dielectric breakdown in $\mathrm{MgO}$ based magnetic tunnel junctions, Applied Physics Letters 93, 152508 (2008).

[35] G.-X. Miao, M. Münzenberg und J. S. Moodera, Tunneling path toward spintronics, Reports on Progress in Physics 74, 036501 (2011).

[36] W. H. Butler, Tunneling magnetoresistance from a symmetry filtering effect, Science and Technology of Advanced Materials 9, 024207 (2008). 
[37] S. Yuasa, T. Nagahama, A. Fukushima, Y. Suzuki und K. Ando, Giant room-temperature magnetoresistance in single-crystal $\mathrm{Fe} / \mathrm{MgO} / \mathrm{Fe}$ magnetic tunnel junctions, Nature Materials 3, 868 (2004).

[38] P. Mavropoulos, N. Papanikolaou und P. H. Dederichs, Complex Band Structure and Tunneling through Ferromagnet / Insulator / Ferromagnet Junctions, Phys. Rev. Lett. 85, 1088 (2000).

[39] X.-G. Zhang und W. H. Butler, Large magnetoresistance in bcc $\mathrm{Co} / \mathrm{MgO} / \mathrm{Co}$ and $\mathrm{FeCo} / \mathrm{MgO} / \mathrm{FeCo}$ tunnel junctions, Physical Review B 70, 172407 (2004).

[40] N. W. Ashcroft und D. M. Mermin, Festkörperphysik, Oldenbourg Verlag, München, Wien, 3. Edition, 2007.

[41] P. Villars und L. D. Calvert, Pearson's Handbook of Crystallographic Data for Intermetallic Phases, ASM International, 1991.

[42] W. Wulfhekel, M. Klaua, D. Ullmann, F. Zavaliche, J. Kirschner, R. Urban, T. Monchesky und B. Heinrich, Single-crystal magnetotunnel junctions, 78, 509 (2001).

[43] A. Meschede und H.-U. Krebs, Minimization of interface roughness in laser deposited Fe/MgO multilayers, Appl. Phys. A 102, 137 (2011).

[44] M. Gradhand, C. Heiliger, P. Zahn und I. Mertig, Tunneling magnetoresistance with amorphous electrodes, Phys. Rev. B 77, 134403 (2008).

[45] G. Eilers, Korrelationen zwischen struktureller Ordnung und elektrischen Transporteigenschaften in $\mathrm{CoFeB} / \mathrm{Mg}-\mathrm{B}-\mathrm{O} / \mathrm{CoFeB}$ Tunnelmagnetowiderstandselementen, Dissertation, Universitaet Goettingen, 2009.

[46] Y. S. Choi, K. Tsunekawa, Y. Nagamine und D. Djayaprawira, Transmission electron microscopy study on the polycrystalline Co$\mathrm{FeB} / \mathrm{MgO} / \mathrm{CoFeB}$ based magnetic tunnel junction showing a high tunneling magnetoresistance, predicted in single crystal magnetic tunnel junction, Journal of Applied Physics 101, 013907 (2007).

[47] E. A. Colbourn, Computer simulation of defects and reactions at oxide surfaces, Surface Science Reports 15, 281 (1992).

[48] T. Ibusuki, T. Miyajima, S. Umehara, S. Eguchi und M. Sato, Lowertemperature crystallization of $\mathrm{CoFeB}$ in $\mathrm{MgO}$ magnetic tunnel junctions by using Ti capping layer, Applied Physics Letters 94 (2009). 
[49] Y. Lu, C. Deranlot, A. Vaures, F. Petroff, J.-M. George, Y. Zheng und D. Demailles, Effects of a thin Mg layer on the structural and magnetoresistance properties of $\mathrm{CoFeB} / \mathrm{MgO} / \mathrm{CoFeB}$ magnetic tunnel junctions, Applied Physics Letters 91 (2007).

[50] H. Kurt, K. Rode, K. Oguz, M. Boese, C. C. Faulkner und J. M. D. Coey, Boron diffusion in magnetic tunnel junctions with $M g O$ (001) barriers and CoFeB electrodes, Applied Physics Letters 96, 262501 (2010).

[51] N. I. Borgardt, B. Plikat und M. Seibt, High-Resolution Electron Microscopy of Interfaces between Solids with Varying Degree of Atomic Ordering, Interface Science 12, 311 (2004).

[52] B. Plikat, B. Borgardt, M. Seibt, W. Schröter und T.Wagner, The Modified Structure of Amorphous Ge near Si(111) Substrates, Inst. Phys. Conf. Ser. 164, 43 (1999).

[53] K. Thiel, N. Borgardt, B. Plikat und M. Seibt, Mesoscopic properties of interfacial ordering in amorphous germanium on Si(111) determined by quantitative digital image series matching, Ultramicroscopy 126, 1 (2013).

[54] D. B. Williams und C. B. Carter, Transmission Electron Microscopy A Textbook for Material Science, Springer, 2. Edition, 2009.

[55] J. C. H. Spence, High-Resolution Electron Microscopy, Oxford University Press, 2003.

[56] M. De Graef, Introduction to Conventional Transmission Electron Microscopy, Cambridge University Press, 2003.

[57] J. M. Cowley, Twenty forms of electron holography, Ultramicroscopy 41, 335 (1992).

[58] M. Lehmann, Quantitative Electron Holography with Atomic Resolution (Habitilationsschrift), Dissertation, T.U. Dresden, 2004.

[59] R. Erni, Aberration-Corrected Imaging in Transmission Electron Microscopy, Imperial College Press, 2010.

[60] O. Scherzer, Über einige Fehler von Elektronenlinsen, Zeitschrift für Physik 101, 593 (1936). 
[61] M. Born und E. Wolf, Principles of Optics, Cambridge University Press, 2001.

[62] H. Rose, Outline of a spherically corrected semiaplanatic medium-voltage transmission electron-microscope, Optik 85, 19 (1990).

[63] M. Haider, H. Rose, S. Uhlemann, B. Kabius und K. Urban, Towards 0.1 $n m$ resolution with the first spherically corrected transmission electron microscope, Journal of Electron Microscopy 47, 395 (1998).

[64] F. Zemlin, K. Weiss, P. Schiske, W. Kunath und K.-H. Herrmann, Comafree alignment of high resolution electron microscopes with the aid of optical diffractograms, Ultramicroscopy 3, 49 (1978).

[65] S. Uhlemann und M. Haider, Residual wave aberrations in the first spherical aberration corrected transmission electron microscope, Ultramicroscopy 72, 109 (1998).

[66] C.-L. Jia, M. Lentzen und K. Urban, High-Resolution Transmission Electron Microscopy Using Negative Spherical Aberration, Microscopy and Microanalysis 10, 174 (2004).

[67] C. L. Jia und K. Urban, Atomic-Resolution Measurement of Oxygen Concentration in Oxide Materials, Science 303, 2001 (2004).

[68] K. W. Urban, C.-L. Jia, L. Houben, M. Lentzen, S.-B. Mi und K. Tillmann, Negative spherical aberration ultrahigh-resolution imaging in corrected transmission electron microscopy, Philosophical Transactions of the Royal Society A: Mathematical,Physical and Engineering Sciences 367, 3735 (2009).

[69] D. J. Smith, Progress and perspectives for atomic-resolution electron microscopy, Ultramicroscopy 108, 159 (2008), Proceedings of the Sixteenth International Microscopy Congress.

[70] C. Kisielowski, C. J. D. Hetherington, Y. C. Wang, R. Kilaas, M. A. O'Keefe und A. Thust, Imaging columns of the light elements carbon, nitrogen and oxygen with sub Angstrom resolution, Ultramicroscopy 89, 243 (2001).

[71] K. Tillmann, L. Houben, A. Thust und K. Urban, Spherical-aberration correction in tandem with the restoration of the exit-plane wavefunction: synergetic tools for the imaging of lattice imperfections in crystalline 
solids at atomic resolution, Journal of Materials Science 41, 4420 (2006), 10.1007/s10853-006-0154-0.

[72] W. M. J. Coene, A. Thust, M. O. de Beeck und D. V. Dyck, Maximumlikelihood method for focus-variation image reconstruction in high resolution transmission electron microscopy, Ultramicroscopy 64, 109 (1996).

[73] A. Thust, W. M. J. Coene, M. O. de Beeck und D. V. Dyck, Focal-series reconstruction in HRTEM: simulation studies on non-periodic objects, Ultramicroscopy 64, 211 (1996).

[74] A. Thust, M. Overwijk, W. Coene und M. Lentzen, Numerical correction of lens aberrations in phase-retrieval $\{H R T E M\}$, Ultramicroscopy 64, 249 (1996).

[75] C. Kübel und A. Thust, TrueImage: Introduction to Focal-Series Reconstruction, Springer, 2005.

[76] M. O. de Beeck, D. V. Dyck und W. Coene, Wave function reconstruction in HRTEM: the parabola method, Ultramicroscopy 64, 167 (1996).

[77] W. Saxton, What is the focus variation method? Is it new? Is it direct?, Ultramicroscopy 55, 171 (1994).

[78] J. Kirkland, Improved high resolution image processing of bight field electron micrographs I. Theory, Ultramicroscopy 15, 151 (1984).

[79] M. Lentzen und K. Urban, Reconstruction of the projected crystal potential in transmission electron microscopy by means of a maximumlikelihood refinement algorithm, Acta Crystallographica Section A 56, 235 (2000).

[80] R. Rosenfeld, A. Thust, W. Yang, M. Feuerbacher und K. Urban, Investigation of the cluster substructure of icosahedral Al-Pd-Mn quasicrystals by means of exit-plane wavefunction reconstruction in high-resolution electron microscopy, Philosophical Magazine Letters 78, 127 (1998).

[81] C. L. Jia, Atomic structure of a $\Sigma=3,111$ twin-boundary junction in a $\mathrm{BaTiO}_{3}$ thin film, Philosophical Magazine Letters 79, 99 (1999).

[82] C. L. Jia und A. Thust, Investigation of Atomic Displacements at a $\Sigma 3$ 111 Twin Boundary in $\mathrm{BaTiO}_{3}$ by Means of Phase-Retrieval Electron Microscopy, Phys. Rev. Lett. 82, 5052 (1999). 
[83] G. Cliff und G. Lorimer, The quantitative analysis of thin specimens, J. Microscopy 103, 203 (1975).

[84] R. F. Egerton, Electron Energy-Loss Spectroscopy in the Electron Microscope, Springer US, 2011.

[85] H. Sauer, R. Brydson, P. Rowley, W. Engel und J. Thomas, Determination of coordinations and coordination-specific site occupancies by electron energy-loss spectroscopy: An investigation of boron-oxygen compounds, Ultramicroscopy 49, 198 (1993).

[86] J. Hosoi, T. Oikawa, M. Inoue, Y. Kokubo und K. Hama, Measurement of partial specific thickness (net thickness) of critical-point-dried cultured fibroblast by energy analysis, Ultramicroscopy 7, 147 (1981).

[87] C.-W. Lee, Y. Ikematsu und D. Shindo, Measurement of mean free paths for inelastic electron scattering of Si and SiO2, Journal of Electron Microscopy 51, 143 (2002).

[88] R. D. Leapman und D. E. Newbury, Trace elemental analysis at nanometer spatial resolution by parallel-detection electron energy loss spectroscopy, Analytical Chemistry 65, 2409 (1993), PMID: 8238934.

[89] G. Möbus, R. Schweinfest, T. Gemming, T. Wagner und M. Rühle, Iterative structure retrieval techniques in HREM: a comparative study and a modular program package, Journal of Microscopy 190, 109 (1998).

[90] G. Möbus, Structure Determination by Quantitative High-Resolution Transmission Electron Microscopy, in High-Resolution Imaging and Spectrometry of Materials, herausgegeben von F. Ernst und M. Ruehle, Band 50 der Springer Series in Materials Science, Seiten 69-118, Springer Berlin Heidelberg, 2003.

[91] P. Stadelmann, EMS - a software package for electron diffraction analysis and HRTEM image simulation in materials science, Ultramicroscopy $\mathbf{2 1}$, 131 (1987).

[92] G. Eilers, H. Ulrichs, M. Münzenberg, A. Thomas, K. Thiel und M. Seibt, Long-range order on the atomic scale induced at $\mathrm{CoFeB} / \mathrm{MgO}$ interfaces, J. Appl. Phys. 105 (2009).

[93] L. Al-Ali, Translational alignment of didifferent defocused micrographs using cross-correlation, in Developments in Electron Microscopy and Analysis, Seiten 225-228, J. A. Venables, 1976. 
[94] M. Hÿtch und W. Stobbs, Quantitative criteria for the matching of simulations with experimental HREM images, Microsc. Microanal. Microstruct. 5, 133 (1994).

[95] M. Hÿtch und W. Stobbs, Quantitative comparison of high resolution TEM images with image simulations, Ultramicroscopy 53, 191 (1994).

[96] C. B. Boothroyd, Why don't high-resolution simulations and images match?, Journal of Microscopy 190, 99 (1998).

[97] C. Boothroyd, Quantification of high-resolution electron microscope images of amorphous carbon, Ultramicroscopy 83, 159 (2000).

[98] C. B. Boothroyd, Quantification of lattice images: the contribution from diffuse scattering, Journal of Electron Microscopy 51, S279 (2002).

[99] A. Howie, Hunting the Stobbs factor, Ultramicroscopy 98, 73 (2004), Festschrift in Honor of the 70th Birthday of Professor Fang-Hua Li.

[100] J. M. Howe, Direct observation of order in the liquid at a solid-liquid interface by high-resolution transmission electron microscopy, Philosophical Magazine A 74, 761 (1996).

[101] Y. Luo, M. Esseling, A. Käufler, K. Samwer, T. Dimopoulos, G. Gieres, M. Vieth, M. Rührig, J. Wecker, C. Rudolf, T. Niermann und M. Seibt, Co-rich magnetic amorphous films and their application in magnetoelectronics, Phys. Rev. B 72, 014426 (2005).

[102] T. Niermann, Bestimmung der Modulationstransferfunktion, private Mitteilung, 2013.

[103] A. Thust, High-Resolution Transmission Electron Microscopy on an Absolute Contrast Scale, Phys. Rev. Lett. 102, 220801 (2009).

[104] H. Schuhmann, Transmissionselektronenmikroskopische Untersuchungen zur Mikrostruktur von InN-Nanodrähten unter Verwendung unterschiedlicher Probenpräparationstechniken, Master's thesis, IV. Physikalisches Institut der Georg-August-Universität Göttingen, 2009.

[105] M. Süess, E. Mueller und R. Wepf, Minimization of amorphous layer in Ar+ ion milling for UHR-EM, Ultramicroscopy 111, 1224 (2011).

[106] J. Dean und N. Lange, Lange's handbook of chemistry, Lange's Handbook of Chemistry, McGraw-Hill, 1992. 
[107] C. Wang, A. Kohn, S. G. Wang, L. Y. Chang, S.-Y. Choi, A. I. Kirkland, A. K. Petford-Long und R. C. C. Ward, Structural characterization of interfaces in epitaxial $\mathrm{Fe} / \mathrm{MgO} / \mathrm{Fe}$ magnetic tunnel junctions by transmission electron microscopy, Phys. Rev. B 82, 024428 (2010).

[108] Wilson und Prince, editors, International Tables For Crysallography, Kluwer Academic Publischers, 1999.

[109] B. Efron, Bootstrap Methods: Another Look at the Jackknife, The Annals of Statistics 7, pp. 1 (1979).

[110] B. Efron und R. Tibshirani, Bootstrap Methods for Standard Errors, Confidence Intervals, and Other Measures of Statistical Accuracy, Statistical Science 1, pp. 54 (1986).

[111] J. A. Nelder und R. Mead, A simplex method for function minimization, Computer Journal 7, 308 (1965).

[112] W. Press, W. Vetterling, S. Teukolsky und B. Flannery, Numerical Recipes in C, Cambridge University Press, 2. edition Edition, 1992.

[113] C. Wang, S. Wang, A. Kohn, R. Ward und A. Petford-Long, Transmission Electron Microscopy Study of the Fe(001) / MgO(001) Interface for Magnetic Tunnel Junctions, Magnetics, IEEE Transactions on 43, 2779 (2007).

[114] H. L. Meyerheim, R. Popescu, N. Jedrecy, M. Vedpathak, M. SauvageSimkin, R. Pinchaux, B. Heinrich und J. Kirschner, Surface $x$-ray diffraction analysis of the $\mathrm{MgO} / \mathrm{Fe}(001)$ interface: Evidence for an $\mathrm{FeO}$ layer, Phys. Rev. B 65, 144433 (2002).

[115] H. L. Meyerheim, R. Popescu, J. Kirschner, N. Jedrecy, M. SauvageSimkin, B. Heinrich und R. Pinchaux, Geometrical and Compositional Structure at Metal-Oxide Interfaces: $\mathrm{MgO}$ on Fe(001), Phys. Rev. Lett. 87, 076102 (2001).

[116] B. D. Yu und J.-S. Kim, Abinitio study of ultrathin $M g O$ films on Fe(001): Influence of interfacial structures, Phys. Rev. B 73, 125408 (2006).

[117] H. Schuhmann, M. Seibt, V. Drewello, A. Thomas, V. Zbarsky, M. Walter und M. Münzenberg, Crystallization and boron redistribution in $\mathrm{CoFeB} / \mathrm{MgO}$ systems for magnetic tunnel junctions, unpublished, 2014. 
[118] C. C. Ahn und O. L. Krivanek, EELS Atlas, Gatan, Inc., 1983.

[119] M. J. Plisch, J. L. Chang, J. Silcox und R. A. Buhrman, Atomic-scale characterization of a Co/AlO $/$ Co magnetic tunnel junction by scanning transmission electron microscopy, Applied Physics Letters 79, 391 (2001).

[120] J. P. Velev, K. D. Belashchenko, S. S. Jaswal und E. Y. Tsymbal, Effect of oxygen vacancies on spin-dependent tunneling in $\mathrm{Fe} / \mathrm{MgO} / \mathrm{Fe}$ magnetic tunnel junctions, Applied Physics Letters 90, 072502 (2007).

[121] V. Harnchana, A. T. Hindmarch, M. C. Sarahan, C. H. Marrows, A. P. Brown und R. M. D. Brydson, Evidence for boron diffusion into substoichiometric $\mathrm{MgO}$ (001) barriers in $\mathrm{CoFeB} / \mathrm{MgO}$-based magnetic tunnel junctions, Journal of Applied Physics 113, (2013).

[122] M. Schäfers, V. Drewello, G. Reiss, A. Thomas, K. Thiel, G. Eilers, M. Münzenberg, H. Schuhmann und M. Seibt, Electric breakdown in ultrathin $\mathrm{MgO}$ tunnel barrier junctions for spin-transfer torque switching, Applied Physics Letters 95, 232119 (2009).

[123] P. Peretzki, Untersuchung der Textur von MgO-CoFeBDünnschichtsystemen mittles hochauflösender Transmissionselektronenmikroskopie, Master's thesis, Universität Göttingen, 2011.

[124] A. Weickenmeier und H. Kohl, Computation of absorptive form factors for high-energy electron diffraction, Acta Crystallographica Section A 47, 590 (1991). 


\section{Danksagung}

Mein Dank gilt als erstes meinem Doktorvater Prof. Dr. Michael Seibt für die Ermöglichung dieser Doktorarbeit sowie den hilfreichen Diskussionen, Kritiken und Ratschlägen.

Für die Übernahme des Korreferats danke ich Herrn Prof. Dr. Christian Jooß.

Prof. Dr. Nikolai Borgardt möchte ich für die unermüdliche Unterstützung über die letzten Jahre danken. Seine ständige Diskussionsbereitschaft während seiner kurzen Aufenthalte in Göttingen hat zum steten Fortschritt dieser Arbeit beigetragen.

Ebenso möchte ich mich bei Dr. Karsten Thiel für seine Hilfsbereitschaft danken.

Prof. Dr. Markus Münzenberg sowie seiner Arbeitsgruppe, namentlich Marvin Walter und Vladyslav Zbarsky, möchte ich für die Probenherstellung danken.

Danken möchte ich ebenfalls Volker Radisch für die technische Unterstützung am FIB sowie Matthias Hahn für die Hilfe bei technischen Problemen am neuen ETEM.

Für die angenehme Arbeitsatmosphäre möchte ich mich insbesondere bei meinen langjährigen Zimmergenossen Sarina Grevsmühl und Patrick Schwager bedanken. Außerdem danke ich der gesamten Arbeitsgruppe die zur Zeit aus Marie Aylin Falkenberg, Philipp Saring, Patrick Peretzki, Nan Wang, Juliane Clemens, Arne Ahrens, Corin Jung sowie Jens Lunte besteht für die ständige Diskussionsbereitschaft und deren Hilfsbereitschaft.

Für die angenehme Zusammenarbeit danke ich allen Mitgliedern des IV. Physikalischen Instituts.

Meinem Bruder, Dr. Holger Schuhmann, danke ich für die Durchsicht des Manuskriptes.

Zum Schluss danke ich meinen Eltern für die Ermöglichung des Studiums und die fortwährende Unterstützung.

Mein größter Dank gilt jedoch meiner Frau Sandra, nicht nur für die sprachlichen Korrekturen des Manuskripts, sondern v.a. für die andauernde Unterstützung und das Aushalten der entbehrungsreichen Jahre. 



\section{Lebenslauf}

\section{Persönliche Angaben}

Name: Henning Schuhmann

Familienstand: verheiratet

Staatsangehörigkeit: deutsch

Geburtsdatum: 06. Januar 1980

Geburtsort: Wilhelmshaven

Schulausbildung

$\begin{array}{ll}1986-1990 & \text { Grundschule Coldewei } \\ 1990-1992 & \text { Orientierungsstufe Salzastraße } \\ 1992-1999 & \text { Gymnasium Am Mühlenweg }\end{array}$

Wehrdienst

$1999-2000 \quad$ Grundwehrdienst in Oldenburg

Hochschulausbildung

WiSe 2000/2001 Aufnahme des Studiums der Physik an der Georg-August-Universität Göttingen

04/2009 Diplom, Titel der Arbeit: Transmissionselektronenmikroskopische Untersuchungen zur Mikrostruktur von InN-Nanodrähten unter Verwendung unterschiedlicher Probenpräparationstechniken

seit 05/2009 Promotion unter der Betreuung von Prof. Dr. Michael Seibt an der Georg-August Universität Göttingen 
